LBNL-43337

\title{
Small Numbers in Supersymmetric Theories of Nature
}

\author{
Michael Lawrence Graesser \\ Ph.D. Thesis \\ Department of Physics \\ University of California, Berkeley \\ and \\ Physics Division \\ Ernest Orlando Lawrence Berkeley National Laboratory \\ University of California \\ Berkeley, CA 94720
}

May 1999

This work was supported by the Director, Office of Energy Research, Office of High Energy and Nuclear Physics, Division of High Energy Physics, of the U.S. Department of Energy under Contract No. DE-AC03$76 \mathrm{SF} 00098$. 


\section{DISCLAIMER}

This report was prepared as an account of work sponsored by an agency of the United States Government. Neither the United States Government nor any agency thereof, nor any of their employees, make any warranty, express or implied, or assumes any legal liability or responsibility for the accuracy, completeness, or usefulness of any information, apparatus, product, or process disclosed, or represents that its use would not infringe privately owned rights. Reference herein to any specific commercial product, process, or service by trade name, trademark, manufacturer, or otherwise does not necessarily constitute or imply its endorsement, recommendation, or favoring by the United States Government or any agency thereof. The views and opinions of authors expressed herein do not necessarily state or reflect those of the United States Government or any agency thereof. 


\section{DISCLAIMER}

Portions of this document may be illegible in electronic image products. Images are produced from the best available original document. 


\title{
Small Numbers in Supersymmetric Theories of Nature
}

\author{
by
}

Michael Lawrence Graesser

B.S. (University of Toronto, Toronto, Canada) 1994

A dissertation submitted in partial satisfaction of the

requirements for the degree of

Doctor of Philosophy

in

Physics

in the

GRADUATE DIVISION

of the

UNIVERSITY of CALIFORNIA, BERKELEY

Committee in charge:

Professor Mahiko Suzuki, Co-Chair

Dr. Ian Hinchliffe, Co-Chair

Professor Lawrence Hall

Professor Nicolai Reshetikhin

Spring 1999 


\section{Small Numbers in Supersymmetric Theories of Nature}

Copyright $(\subset 1999$

by

Michael Lawrence Graesser

The U.S. Department of Energy has the right to use this document for any purpose whatsoever including the right to reproduce all or any part thereof. 


\title{
Abstract \\ Small Numbers in Supersymmetric Theories of Nature
}

\author{
by \\ Michael Lawrence Graesser \\ Doctor of Philosophy in Physics \\ University of California, Berkeley \\ Professor Mahiko Suzuki, Co-Chair \\ Dr. Ian Hinchliffe, Co-Chair
}

The Standard Model of particle interactions is a successful theory for describing the interactions of quarks, leptons and gauge bosons at microscopic distance scales. Despite these successes, the theory contains many unsatisfactory features. The origin of particle masses is a central mystery that has eluded experimental elucidation. In the Standard Model the known particles obtain their mass from the condensate of the so-called Higgs particle. Quantum corrections to the Higgs mass require an unnatural fine tuning in the Higgs mass of one part in $10^{-32}$ to obtain the correct mass scale of electroweak physics. In addition, the origin of the vast hierarchy between the mass scales of the electroweak and quantum gravity physics is not explained in the current theory. 
Supersymmetric extensions to the Standard Model are not plagued by this fine tuning issue and may therefore be relevant in Nature. In the minimal supersymmetric Standard Model there is also a natural explanation for electroweak symmetry breaking. Supersymmetric Grand Unified Theories also correctly predict a parameter of the Standard Model. This provides non-trivial indirect evidence for these theories.

The most general supersymmetric extension to the Standard Model however, is excluded by many physical processes, such as rare flavor changing processes, and the non-observation of the instability of the proton. These processes provide important information about the possible structure such a theory. In particular, certain parameters in this theory must be rather small. A physics explanation for why this is the case would be desirable.

It is striking that the gauge couplings of the Standard Model unify if there is supersymmetry close to the weak scale. This suggests that at high energies Nature is described by a supersymmetric Grand Unified Theory. But the mass scale of unification must be introduced into the theory since it does not coincide with the probable mass scale of strong quantum gravity.

The subject of this dissertation is both the phenomenology and model-building opportunities that may lie behind the small numbers that appear in supersymmetric extensions of the Standard Model. 
To Mom and Dad 


\section{Contents}

1 Introduction $\quad 1$

2 Non-renormalization Theorem for the Wilsonian Gauge Couplings in Supersymmetric Theories $\quad 40$

2.1. Introduction . . . . . . . . . . . . . . 40

2.2 Simple Gauge Group . . . . . . . . . . . . . . . . 44

2.3 Extension to a semi-simple gauge group . . . . . . . . . 50

$3 \quad R$-Parity Violation $\quad 54$

3.1 Introduction . . . . . . . . . . . . . . 54

3.2 Flavor Changing Neutral Current Processes . . . . . . . . . 57

3.3 Top Quark Decay . . . . . . . . . . . . . . 66

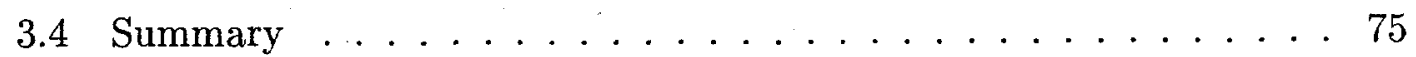

4 Signals of Supersymmetric Flavor Violation at the LHC 79

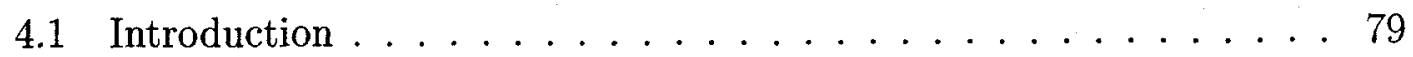

4.2 Slepton Production by Drell-Yan Process . . . . . . . . . . . . 85

4.3 Slepton Production in Cascade Decays . . . . . . . . . . 89

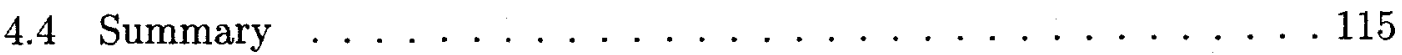

5 Finetuning in Low-Energy Gauge Mediation $\quad 116$ 


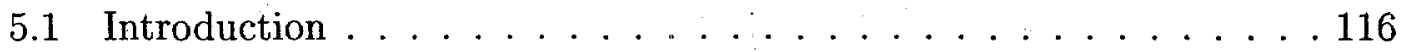

5.2 Messenger Sector . . . . . . . . . . . . . . . . 120

5.3 Fine Tuning in the Minimal LEGM $\ldots \ldots \ldots \ldots$

5.4 A Toy Model to Reduce. Fine Tuning . . . . . . . . . . . . 129

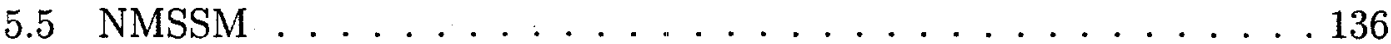

5.6 Models Derived from a GUT . . . . . . . . . . . . . . 147

5.7 One complete Model $\ldots \ldots \ldots \ldots \ldots \ldots \ldots$

5.8 Summary . . . . . . . . . . . . . . . . . . 159

6 Non-decoupling of the First and Second Generation Scalars 163

6.1 Introduction . . . . . . . . . . . . . 164

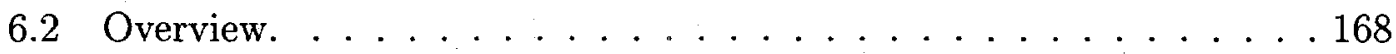

6.3 Low Energy Supersymmetry Breaking . . . . . . . . . . . . . . 182

6.4 High Scale Supersymmetry Breaking . . . . . . . . . . . 193

6.5 Using Finetuning to Constrain $\delta \ldots \ldots \ldots \ldots \ldots \ldots \ldots$

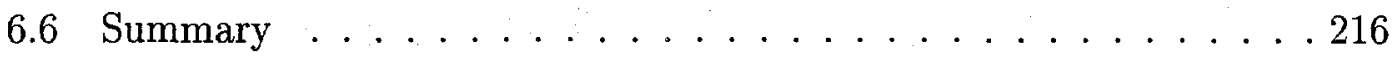

7 Dynamically Generating the Grand Unification Scale 221

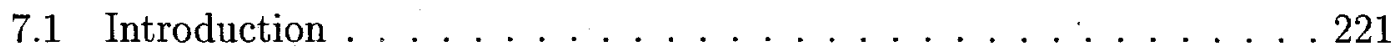

7.2 Overview . . . . . . . . . . . . . . . . 224

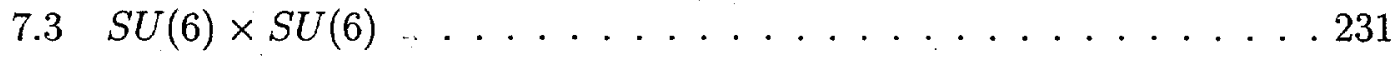

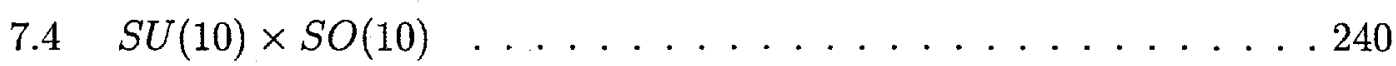

7.5 Summary . . . . . . . . . . . . . . 254 
A Fine tuning Expressions

B Two-loop calculation

263

C Spectrum of $S U(6) \times S U(6)_{G U T}$

269

D Spectrum of $S U(10) \times S O(10)_{G U T}$ Model

272 


\section{List of Figures}

1.1 Feynman diagrams contributing quadratic divergences to the Higgs mass. Solid, dotted and wavy lines represent propagating fermions, scalars and gauge bosons, respectively. ............. 6

1.2 Cancellation in supersymmetric theories of quadratic divergences to the scalar masses. Solid and dotted lines represent propagating fermions and bosons respectively. ................... 11

$1.3 R$-parity violating proton decay $p \rightarrow \pi^{0} e^{+} \ldots \ldots \ldots . \ldots 17$

1.4 A supersymmetric contribution to $\mu \rightarrow e \gamma$. Here $\tilde{\mu}$ is a smuon, $\tilde{e}$ is a selectron, and $\tilde{N}^{0}$ is a neutralino. The "cross" indicates a $\tilde{\mu} \rightarrow \tilde{e}$

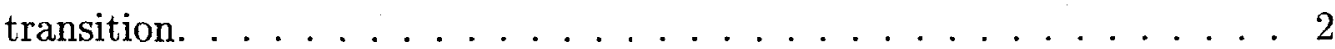

1.5 A supersymmetric contribution to $K-\bar{K}$ mixing. Here $\tilde{s}$ is a strange squark, $\tilde{d}$ is a down squark, and $\tilde{g}$ is a gluino. The crosses indicate $\tilde{s} \leftrightarrow \tilde{d}$ transitions. . . . . . . . . . . . . . . . . . . . 2

1.6 Scaling of gauge couplings in the Standard Model. The band corresponds to the $1 \sigma$ error $(0.003)$ on $\alpha_{3}\left(M_{Z}\right)$. The couplings do not

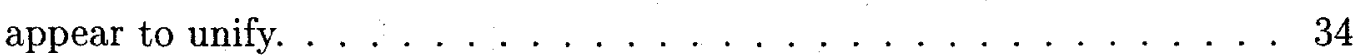

1.7 Scaling of the gauge couplings in the Minimal Supersymmetric Standard Model. The band corresponds to the $1 \sigma$ error $(0.003)$ on $\alpha_{3}\left(M_{Z}\right)$. The couplings do appear to unify. . . . . . . . . . . . . 35 
1.8 Superfield Feynman diagram from colored higgsino exchange leading to proton decay. The "cross" indicates that there is some mass mixing between the two colored higgsinos. $\ldots \ldots \ldots \ldots \ldots \ldots$

$3.1 \not \lambda_{p}$ contributions to $K^{0}-\bar{K}^{0}$ mixing with one $\lambda_{i j k}^{\prime} \neq 0$. Arrows indicate flow of propagating left handed fields. . . . . . . . . . 61

$3.2 \not R_{p}$ contribution to $K^{+} \rightarrow \pi^{+} \nu \bar{\nu}$ with one $\lambda_{i j k}^{\prime} \neq 0 . \ldots \ldots \ldots 64$

5.1 Contours of $c\left(m_{Z}^{2} ; \mu^{2}\right)=(10,15,20,25,40,60)$ for a MSSM with a messenger particle content of one $(5+\overline{5})$. In figures $(a)$ and $(c)$ $\operatorname{sgn}(\mu)=-1$ and in figures $(b)$ and $(d) \operatorname{sgn}(\mu)=+1$. The constraints considered are: (I) $m_{\tilde{e}_{R}}=75 \mathrm{GeV}$, (II) $m_{\tilde{\chi}_{1}^{0}}+m_{\tilde{\chi}_{2}^{0}}=160 \mathrm{GeV}$, (III) $m_{\tilde{e}_{R}}=85 \mathrm{GeV}$, and (IV) $m_{\tilde{\chi}_{1}^{0}}+m_{\tilde{\chi}_{2}^{0}}=180 \mathrm{GeV} \ldots \ldots \ldots \ldots$

5.2 Contours of $c\left(m_{Z}^{2} ; \mu^{2}\right)=(1,2,3,5,7,10)$ for a MSSM with a messenger particle content of three $(l+\bar{l})$ 's and one $(q+\bar{q})$. In figures $(a)$ and $(c)$ $\operatorname{sgn}(\mu)=-1$ and in figures $(b)$ and $(d) \operatorname{sgn}(\mu)=+1$. The constraints considered are: (I) $m_{\tilde{e}_{R}}=75 \mathrm{GeV}$, (II) $m_{\tilde{\chi}_{1}^{0}}+m_{\tilde{\chi}_{2}^{0}}=160 \mathrm{GeV}$, (III) $m_{\tilde{e}_{R}}=85 \mathrm{GeV}$, and (IV) $m_{\tilde{\chi}_{1}^{0}}+m_{\tilde{\chi}_{2}^{0}}=180 \mathrm{GeV} \ldots \ldots \ldots \ldots 133$ 
5.3 Contours of $c\left(m_{Z}^{2} ; \lambda_{H}\right)$ for the NMSSM of Section 3.5 and a messenger particle content of three $(l+\bar{l})$ 's and one $(q+\bar{q})$. In figures $(a)$ and (b), $c\left(m_{Z}^{2} ; \lambda_{H}\right)=(4,5,6,10,15)$ and $\lambda_{H}=0.1$. In figures $(c)$ and $(d)$, $c\left(m_{Z}^{2} ; \lambda_{H}\right)=(3,4,5,10,15,20)$ and $\lambda_{H}=0.5$. The constraints considered are: (I) $m_{h}+m_{a}=m_{Z}$, (II) $m_{\tilde{e}_{R}}=75 \mathrm{GeV}$, (III) $m_{\tilde{\chi}_{1}^{0}}+m_{\tilde{\chi}_{2}^{0}}=160$ $\mathrm{GeV},(\mathrm{IV}) m_{h}=92 \mathrm{GeV},(\mathrm{V}) m_{\tilde{e}_{R}}=85 \mathrm{GeV}$, and (VI) $m_{\tilde{\chi}_{1}^{0}}+m_{\tilde{\chi}_{2}^{0}}=$ $180 \mathrm{GeV}$. For $\lambda_{H}=0.5$, the limit $m_{h}>70 \mathrm{GeV}$ constrains $\tan \beta<5$ (independent of $\Lambda_{\text {mess }}$ ) and is thus not shown. . . . . . . . 142

5.4 Contours of $c\left(m_{Z}^{2} ; \lambda_{H}\right)=(50,80,100,150,200)$ for the NMSSM of Section 5 with $\lambda_{H}=0.1$ and a messenger particle content of one $(5+\overline{5})$. The constraints considered are: (I) $m_{h}+m_{a}=m_{Z}$, (II) $m_{\tilde{e}_{R}}=75 \mathrm{GeV}$, (III) $m_{\tilde{\chi}_{1}^{0}}+m_{\tilde{\chi}_{2}^{0}}=160 \mathrm{GeV}$, (IV) $m_{h}=92 \mathrm{GeV}$, (V) $m_{\tilde{e}_{R}}=85 \mathrm{GeV}$, and (VI) $m_{\tilde{\chi}_{1}^{0}}+m_{\tilde{\chi}_{2}^{0}}=180 \mathrm{GeV}$. A central value of $m_{t}=175 \mathrm{GeV}$ is assumed. . . . . . . . . . . . . . . . . . . . . . 148

6.1 Limits for $m_{\tilde{f}_{i}, 0} / M_{S}$ from the requirement that the masses squared are positive at the weak scale, for low-energy supersymmetry breaking. The regions below the curves are excluded. For the case $(2,0)$, the limits for the other squarks are very similar to that for $\tilde{Q}$ and are therefore not shown. 
6.2 Limits for $m_{\tilde{t}^{c}, 0} / M_{S}$ from the requirement that the stop and slepton mass squared are positive at the weak scale. The regions below the curves are excluded. Low-energy gauge-mediated supersymmetry breaking mass relations between the light sparticles and $\tan \beta=2.2$ are assumed. . . . . . . . . . . . . . . . . . 191

6.3 Limits for $m_{\tilde{t}^{c}, 0}$ from the requirement that the stop and slepton mass squared are positive at the weak scale while suppressing $\Delta m_{K}$, for different values of $\left(n_{5}, n_{10}\right)$, and $\left(\delta_{L L}, \delta_{R R}\right)$. The regions below the curves are excluded. Low-energy gauge-mediated supersymmetry breaking mass relations between the light scalars and $\tan \beta=2.2$ are assumed. .194

6.4 Limits for $m_{\tilde{f}_{i}, 0}$ for different values of $\left(n_{5}, n_{10}\right)$ from the requirement that the mass squareds are positive at the weak scale, assuming a supersymmetry breaking scale of $M_{G U T}$. The regions below the lines are excluded.

6.5 Limits for $\tilde{m}_{0} / M_{S}$ from the requirement that the stop masses squared are positive at the weak scale, for $\tan \beta=2.2, A_{t, 0}=0$ and assuming universal scalar masses at $M_{G U T}$ for the stop and Higgs scalars. The regions below the curves are excluded. 
6.6 Limits for $m_{\tilde{t}, 0} / M_{S}, m_{\tilde{t}^{c}, 0} / M_{S}$, from the requirement that the stop masses squared are positive at the weak scale, for $M_{S U S Y}=M_{G U T}$, $\tan \beta=2.2$ and assuming that $m_{H_{u}, 0}^{2}=0$. The value of $A_{t, 0}$ is chosen to maximize the value of the stop soft masses at the weak scale. The regions below the curves are excluded. . . . . . . . . . . . 204

6.7 Limits for $m_{\tilde{t}, 0} / M_{S}, m_{\tilde{t} c, 0} / M_{S}$, from the requirement that the stop masses squared are positive at the weak scale, for $\left(n_{5}, n_{10}\right)=(2,2)$, $M_{S U S Y}=M_{G U T}, \tan \beta=2.2$, and different values of $\tilde{\mu} / M_{S}$. The contours end at that value of $M_{3,0} / M_{S}$ that gives $m_{H_{u}, 0} / M_{S}=0$. The value of $A_{t, 0}$ is chosen to maximize the value of the stop soft masses at the weak scale. The regions below the lines are excluded. . . . . . . 205

6.8 Limits for $m_{\tilde{t}, 0}$ and $m_{\tilde{t}, 0}, m_{\tilde{e}^{c}}$, and $m_{\tilde{L}}$ from the requirement that the masses squared are positive at the weak scale while suppressing $\Delta m_{K}$. It was assumed that $M_{S U S Y}=M_{G U T}, \tan \beta=2.2$ and that $m_{H_{u}, 0}^{2}=0$. The value of $A_{t, 0}$ was chosen to maximize the value of the stop soft masses at the weak scale. The heavy scalars were decoupled at the minimum value allowed by $\Delta m_{K}$. The regions below the lines are excluded. . . . . . . . . . . . . . . . . 208 
6.9 Limits for $m_{\tilde{t}, 0}$ and $m_{\tilde{t}^{c}, 0}$ from the requirement that the stop masses squared are positive at the weak scale while suppressing $\Delta m_{K}$. It was assumed that $M_{S U S Y}=M_{G U T}, \tan \beta=2.2$ and that $m_{H_{u}, 0}^{2}=m_{\tilde{t}^{c}, 0}^{2}$. The value of $A_{t, 0}$ was chosen to maximize the value of the stop soft masses at the weak scale. The heavy scalars were decoupled at the minimum value allowed by $\Delta m_{K}$. The regions below the lines are excluded. . . . . . . . . . . . . . . . . . . 209

6.10 Limits for $m_{\tilde{t}, 0}$ and $m_{\tilde{t}^{c}, 0}$ from the requirement that the stop masses squared are positive at the weak scale while suppressing $\Delta m_{K}$, for $\left(n_{5}, n_{10}\right)=(2,2), \sqrt{\delta_{L L} \delta_{R R}}=0.04$, and different values of $\mu$. The contours terminate at $m_{H_{u}, 0}^{2}=0$. It was assumed that $M_{S U S Y}=M_{G U T}$ and $\tan \beta=2.2$. The value of $A_{t, 0}$ was chosen to maximize the value of the stop soft masses at the weak scale. The heavy scalars were decoupled at the minimum value allowed by $\Delta m_{K}$. The regions below the lines are excluded. . . . . . . . . . . . . . 210

6.11 Limits for $m_{\tilde{t}, 0}, m_{\tilde{t}^{c}, 0}$ from the requirement that the stop masses squared are positive at the weak scale while suppressing $\Delta m_{K}$, for the cases $\left(n_{5}, n_{10}\right)=(2,0)$ and $(0,2)$. It was assumed that $M_{S U S Y}=M_{G U T}$, $\tan \beta=2.2$ and that $m_{H_{u}, 0}^{2}=0$. The value of $A_{t, 0}$ was chosen to maximize the value of the stop soft masses at the weak scale. The heavy scalars were decoupled at the minimum value allowed by $\Delta m_{K}$. The regions below the lines are excluded. . . . . . . . . . 211 
6.12 Maximum value for $\left(\delta_{L L} \delta_{R R}\right)^{1 / 2}$ that is consistent with $\Delta\left(m_{Z}^{2}, M_{3,0}\right)<$ $100, \Delta\left(m_{Z}^{2}, m_{\tilde{t}, 0}^{2}\right)<100$ and $\left(\Delta m_{K}\right)_{S U S Y}<\left(\Delta m_{K}\right)_{\text {exp }}$. Two boundary conditions are considered: $m_{H_{u}, 0}^{2}=0$ (top) and gauge--mediated relations (bottom). Two values for $\tan \beta$ are considered. The value of $A_{t, 0}$ was chosen to maximize the value of the stop masses at the weak scale. 217

6.13 Maximum value for $\delta_{L L}, \delta_{R R}$ that is consistent with $\Delta\left(m_{Z}^{2}, M_{3,0}\right)<10$, $\Delta\left(m_{Z}^{2}, m_{\tilde{t}, 0}^{2}\right)<10$ and $\left(\Delta m_{K}\right)_{S U S Y}<\left(\Delta m_{K}\right)_{e x p}$. It was assumed that $m_{H_{u}, 0}^{2}=0$. Two values for $\tan \beta$ are considered. The value of $A_{t, 0}$ was chosen to maximize the value of the stop masses at the weak scale. . . 218

B.1 Mixed two-loop corrections to the scalar mass. Wavy lines, wavy lines with a straight line through them, solid lines, and dashed lines denote gauge boson, gaugino, fermion and scalar propagators, respectively. The double-line denotes the hypercharge $D$-term propagator. . . . 265 


\section{List of Tables}

1.1 Quantum number charges of one generation of Standard Model particles with a right-handed neutrino, and two Higgs fields, under the Standard Model gauge group $G=S U(3)_{c} \times S U(2)_{L} \times U(1)_{Y}$. Here $Q=\left(u_{L}, d_{L}\right), L=\left(\nu_{L}, e_{L}\right), H=\left(h^{+}, h^{0}\right)$, and $\bar{H}=\left(\bar{h}^{0}, h^{-}\right)$. The electric charge of a particle is $Q_{e m}=Y+T_{3 L}$, where here $T_{3 L}$ is the value of the diagonal generator of $S U(2)_{L}$ acting on $\psi$, e.g. $+1 / 2$ for $u_{L}$ and $-1 / 2$ for $d_{L} \ldots \ldots \ldots \ldots \ldots \ldots \ldots \ldots$

1.2 An anomaly-free "fake" Standard Model. Here $H$ is the Higgs scalar, and all other fields are fermions. The physics of this model (for one generation) is qualitatively similar to that of the Standard Model. But to the author's ability this model cannot be unified into a Grand Unified Theory. . . . . . . . . . . . . . . . . 27

3.1 Constraints on $\left|\lambda_{i j k}^{\prime}\right|$ from:(a) $K^{+} \rightarrow \pi^{+} \nu \bar{\nu}(90 \% C L)$; (b) $b \rightarrow s \nu \bar{\nu}$ $(90 \% C L)$ [49]; (c) $\nu_{e}$ mass $(90 \% C L)[29]$. These constraints were obtained assuming $C K M$-like mixing in the charged $-1 / 3$ quark sector. All limits are for $100 \mathrm{GeV}$ sparticle masses. . . . . . . . . . . . . 77 
3.2 Constraints on $\left|\lambda_{i j k}^{\prime}\right|$ from:(a) charged current universality $(95 \% C L)$ [28]; (b) $\Gamma\left(\pi \rightarrow e \nu_{e}\right) / \Gamma\left(\pi \rightarrow \mu \nu_{\mu}\right)(1 \sigma)$ [28]; (c) $A_{F B}^{e}(1 \sigma)[28]$; (d) $B R\left[\tau \rightarrow \pi \nu_{\tau}\right](95 \% C L)[31] ;\left(\right.$ e) $B R\left[D^{0} \rightarrow K^{-} \mu^{+} \nu_{\mu}\right] / B R\left[D^{0} \rightarrow\right.$ $\left.K^{-} e^{+} \nu_{e}\right](95 \% C L)$ [31]; (f) atomic parity violation and $e D$ asymmetry $(1 \sigma)$ [28]; (g) $\nu_{\mu}$ deep-inelastic scattering $(95 \% C L)$ [28]; (h) partial $Z^{0}$ decay width $(95 \% C L)$ [32]; (i) top quark decay $(95 \% C L) ;(\mathrm{j}) \nu_{e}$ mass $(90 \% \mathrm{CL})$ [29]. All limits are for $100 \mathrm{GeV}$ sparticle masses. . . . . . . 78

4.1 Mass spectrum in $\mathrm{GeV}$ at LHC Point $[55,56]$. Here $\tilde{q}=\tilde{u}, \tilde{d}, \tilde{c}, \tilde{s}$, and

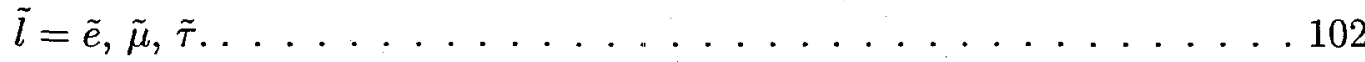

4.2 The production cross-sections in fb for different SUSY particles at the LHC Point $[55,56]$. Here all flavors $\tilde{q}_{H}=\tilde{u}, \tilde{d}, \tilde{c}, \tilde{s}$ and $H=L, R$ are summed over.

4.3 Branching fractions (in percent) for sparticles at LHC Point [56]. Here $\tilde{q}=\tilde{u}, \tilde{d}, \tilde{c}, \tilde{s}$, and $\tilde{l}=\tilde{e}, \tilde{\mu}, \tilde{\tau}$

5.1 Soft scalar masses in GeV for messenger particle content of three $(l+\bar{l})$ 's and one $q+\bar{q}$ and a scale $\Lambda_{\text {mess }}=50 \mathrm{TeV}$.

$5.2 S U(5) \times S U(5)^{\prime} \times Z_{3} \times Z_{3}^{\prime} \times Z_{4}$ quantum numbers for the fields of the model discussed in section 7 . The generators of $Z_{3} \times Z_{3}^{\prime} \times Z_{4}$ are labeled by $(a, b, c)$. The three SM generations are labeled by the index $i \ldots \ldots 160$ 
$5.3 S U(5) \times S U(5)^{\prime} \times Z_{3} \times Z_{3}^{\prime} \times Z_{4}$ quantum numbers for the fields of the model discussed in section 7. The generators of $Z_{3} \times Z_{3}^{\prime} \times Z_{4}$ are labeled by $(a, b, c)$. The three SM generations are labeled by the index $i . \ldots 161$

6.1 Minimum values for heavy scalar masses $M_{S}$ obtained from the measured value of $\Delta m_{K}$ assuming $M_{3}^{2} / M_{S}^{2} \ll 1$. The limits labeled 'QCD incl.' include QCD corrections as discussed in the text. Those labeled

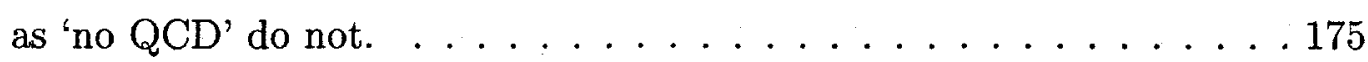

6.2 Minimum values for heavy scalar masses $M_{S}$ obtained from the measured value of $\Delta m_{K}$ assuming $M_{3}^{2} / M_{S}^{2} \ll 1$. The limits labeled as 'QCD incl.' include QCD corrections as discussed in the text. Those labeled as 'no QCD' do not. The limits for $\left(n_{5}, n_{10}\right)=(0,2)$ obtained

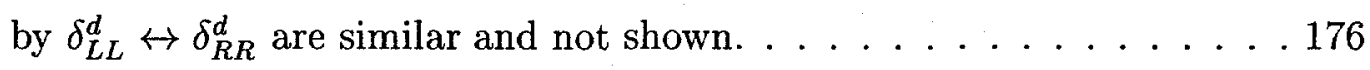




\section{Acknowledgements}

I would like to express my sincere gratitude to my advisors, Professor Mahiko Suzuki and Dr. Ian Hinchliffe, not only for their help in integrating me into this community of scientists, but also for their guidance on, and support of, my research interests. I especially appreciate the freedom they gave me in choosing research topics and the time they lent to the many beneficial discussions. I also thank a fellow student, Kaustubh Agashe, for many rewarding collaborations and discussions.

I would also like to thank other members of the Theoretical Physics group, especially Professor Hitoshi Murayama, Professor Lawrence Hall, Nima ArkaniHamed, Chris Carone, Hsin-Chia Cheng, Csaba Csáki, André de Gouvêa, Chis Kolda, Bogdan Morariu, Takeo Moroi and John Terning for useful discussions and the whole group for a pleasant and rewarding experience of being at LBNL. I appreciate the many values of a community dedicated to learning and striving for truth. I am grateful to Anne Takizawa, Laura Scott and Donna Sakima of the Physics department and Luanne Neumann, Barbara Gordon and Mary Kihanya at LBNL for help with administrative work. I also thank the Natural Sciences and Engineering Research Council of Canada for their support.

I am grateful to my parents for their support ever since I was a child.

And love to Priyanka Kumar for being just like that. 


\section{Chapter 1}

\section{Introduction}

The Standard Model (SM) of particle physics interactions $[1,2]$ is an extremely successful theory of Nature. It qualitatively accounts for many phenomena, such as the meson and baryon mass spectrum, the small measured value for the kaon mass difference, "neutral" current interactions - neutrino-electron scattering, for example - and the weakly interacting nature of quarks at large momentum transfer, to list a few. It also quantitatively agrees with all experimental measurements. The measured anomalous magnetic moment of the electron agrees with the SM prediction to a few parts per billion, and the measured and theoretical values for the anomalous magnetic moment of the muon are consistent to one part per million. These experiments represent tremendous tests of quantum electrodynamics - the part of the SM describing electrons, muons and photons. The SM theory of quarks and gluons - quantum chromodynamics - has also been experimentally tested at SLAC during the 1970's, and at Fermilab in the 1980's. Further, many precise measurements of the SM "weak" interactions performed at the LEP and SLAC experiments in the late 1980's and early 1990's agree with the SM predictions to within their experimental uncertainties (a few percent).

While the SM has many descriptive successes, there are many issues that re- 
main unexplained. The most outstanding issue is the origin of particle masses.

The SM is described by the non-abelian gauge symmetry $S U(3)_{c} \times S U(2)_{L} \times$ $U(1)_{Y}$. The representations of the SM particles under these gauge groups are given in Table 1.1. Of these gauge groups, only the $U(1)_{e m}$ subgroup that corresponds to electromagnetism is observed to be a long-range force. The $S U(3)_{c}$ force (or quantum chromodynamics) is strong at large distances, so it confines quarks into baryons and mesons (e.g. protons, neutrons and pions). The remaining "weak" forces however, have an effective range of about $10^{-16} \mathrm{~cm}$. This is because the $Z$ and $W$ gauge bosons that mediate these "weak" forces are massive. On the other hand, the non-abelian gauge symmetry implies that all the gauge bosons should be massless like the photon. In addition, the leptons and quarks should be massless as well.

The physical mechanism that generates these masses is not known. The particle masses are obtained by either introducing into the theory interactions - such as mass terms for the gauge bosons and fermions - that explicitly break the electroweak symmetry, or by introducing some new interactions whose dynamics spontaneously break the electroweak gauge symmetry. In tile former case the theory is non-renormalizable at the one-loop level, so that the Standard Model is only an effective theory. Further, the preservation of the unitarity of the theory implies that $W W$ interactions should become strong at high energies. In any case, phenomena in the form of new interactions and/or particles should be discovered.

In the second option there must exist some additional undiscovered dynamics 
that is responsible for generating particle masses. There are additional unresolved issues beyond this central mystery. The SM contains six quarks, three charged and neutral neutrinos. The mass of these particles is an input into the theory - the SM does not predict these masses. Since the ratio of the lightest massive particle (the electron) to the heaviest particle (the top quark) is $\approx 10^{-6}$, it is difficult to believe that this small number is part of a fundamental theory of Nature. An explanation for the hierarchies found in the other fermions masses, and the paiameters of the Kobayashi-Maskawa matrix $V_{K M}[3]$, is also desired.

In the Standard Model electroweak symmetry breaking is achieved by introducing a scalar field $H$ with Standard Model quantum numbers $(\mathbf{1}, \mathbf{2}, 1 / 2)$. The scalar potential for $H$ is assumed to be

$$
V(H)=m^{2} H^{a s t} H+\frac{\lambda}{4}\left(H^{*} H\right)^{2}
$$

and is the most general potential that is also gauge invariant and renormalizable. Here $\lambda>0$ so that the potential is bounded from below. The ground state of the vacuum is found by minimizing $V$. In this case the physics of the ground state depends on the sign of $m^{2}$. If $m^{2}>0$ the minimum is at $H=0$. This ground state is invariant under the full SM gauge transformations, so no symmetries are broken at this vacuum. On the other hand, if $m^{2}<0$ there is a local maximum at $H=0$, and the local minimum occurs at a non-zero value for $H$. Using $S U(2)_{L}$ and $U(1)_{Y}$ rotations,

$$
H=\frac{1}{\sqrt{2}}\left(\begin{array}{l}
0 \\
v
\end{array}\right)
$$


minimizes $V$ with $v^{2}=-4 m^{2} / \lambda$. This vacuum is not left invariant by the action of the $S U(2)_{L} \times U(1)_{Y}$ gauge transformations. Only the combination of $S U(2)_{L} \times$ $U(1)_{Y}$ generators $Q=T_{3}+Y$ is left unbroken. Since this is none other than electric charge, this vacuum breaks $S U(2)_{L} \times U(1)_{Y} \rightarrow U(1)_{e m}$. The Higgs mechanism results in a mass of $g v / 2 \approx 80 \mathrm{GeV}$ for the $W$ gauge bosons, and $\sqrt{g^{2}+g^{\prime 2}} v / 2 \approx 90$ $\mathrm{GeV}$ for the $Z$ gauge boson, where $g$ and $g^{\prime}$ are the $S U(2)_{L}$ and $U(1)_{Y}$ gauge couplings, respectively. Numerically $v=247 \mathrm{GeV}$ is determined from the Fermi constant $G_{F}$ obtained from the muon lifetime. Since $U(1)_{e m}$ is left unbroken the photon remains massless. So the symmetry breaking vacuum which corresponds to $m^{2}<0$ correctly describes Nature.

This vacuum expectation value (vev) of the Higgs field can also be used to give masses to the quarks and leptons. The interaction

$$
\lambda_{i j}^{U} Q_{i} H U_{j}^{c}
$$

for example, where $i, j$ are generation labels, is gauge invariant but results in masses for the up-quarks once the vev for $H$ given by Eqn.(1.2) is inserted. Interactions of this type (so-called Yukawa interactions) can also be introduced to give masses to the down-quarks and leptons.

So aside from the fact that the Higgs scalar particle has not been experimentally observed this description of electroweak symmetry breaking is sound. This description however, is theoretically unsatisfactory since the most important step in the story, namely that the Higgs mass parameter $m^{2}$ must be negative, is left 
unexplained. An explanation for why $m^{2}$ is negative, rather than positive, is desired. There are even more distasteful features of this description once quantum corrections to the scalar potential $V$ are considered. These are now described.

In addition to the particle representations and their interactions, a shortdistance cut-off is also required. The physical interpretation for this cut-off is the following. The Standard Model is a good description of Nature at distances above at least $10^{-16} \mathrm{~cm}$, or equivalently, at energies below $100 \mathrm{GeV}$. If the Standard Model were a fundamental theory of Nature, then it would be a good description at all energies. It is not expected for many reasons, however, that this is the case. Firstly, the Standard Model does not explain electroweak symmetry breaking with any satisfaction. It also contains quadratic divergences (discussed in the next paragraph) which are distasteful, and it would be incredibly surprising if they were present in a fundamental theory. It also doesn't explain why the mass scale of electroweak interactions $-100 \mathrm{GeV}$ to a $\mathrm{TeV}-$ is so much smaller than the probable mass scale of (strong) gravitational effects, the Planck scale which is roughly $10^{18} \mathrm{GeV}$. Further, the Compton wavelength and black hole event horizon for a point particle with a mass of $10^{18} \mathrm{GeV}$ coincide, so in describing physical processes at Planck scale energies it is not possible to neglect either quantum or gravitational effects. Both are equally important. But the Standard Model does not include a quantum theory of gravity. For this reason it is expected that at these energies the Standard Model (or any quantum field theory description of Nature) will be replaced by a theory that includes a quantum description of 


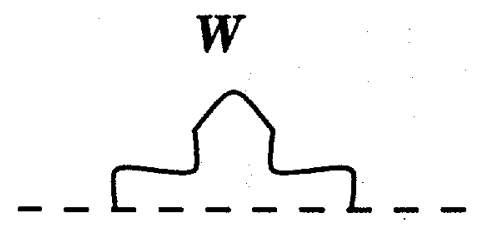

$\boldsymbol{H}$

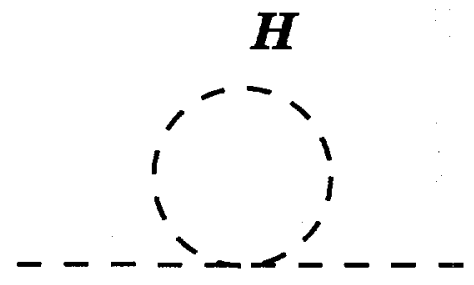

$\boldsymbol{H}$

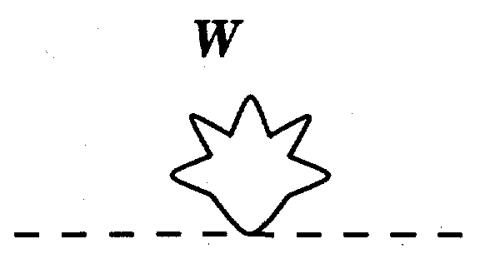

$\boldsymbol{H}$

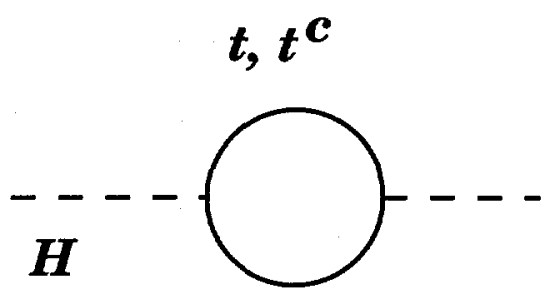

Figure 1.1: Feynman diagrams contributing quadratic divergences to the Higgs mass. Solid, dotted and wavy lines represent propagating fermions, scalars and gauge bosons, respectively.

gravity. So the Standard Model is not a correct description of Nature at these high energies. Thus above some unknown energy scale $\Lambda$ (or below some distance scale $h c / \Lambda)$, the Standard Model is replaced by a better description of Nature. In this sense the Standard Model is only an effective theory of Nature, valid only for energies $E<\Lambda$.

So in evaluating any physical processes it is physically sensible to restrict the energies of the particles to be below $\Lambda$. Of interest for electroweak symmetry breaking are the quantum corrections to the Higgs potential $V$. The interactions of the Higgs scalar with the gauge bosons and with itself correct the expression 
for $V$ given in Eqn.(1.1). In particular, the corrections to the $m^{2}$ parameter are given by the Feynman diagrams in Figure 1.1. As mentioned above, in evaluating these corrections an energy cut-off $\Lambda$ must be introduced. These diagrams give a correction to $m^{2}$ that is

$$
m^{2}=m_{0}^{2}+\frac{C}{16 \pi^{2}} \Lambda^{2} .
$$

Here $m_{0}^{2}$ is the "bare" parameter for the Higgs mass (what was called $m^{2}$ before), and $C$ is a function of the gauge and Yukawa couplings of the theory and is not equal to zero in general. That $\Delta m^{2} \propto \Lambda^{2}$ means that this theory contains a quadratic divergence.

This is distasteful for the following reason. Recall that the gauge boson mass is $m_{V}^{2} \propto v^{2}$, and $v^{2} \propto-m^{2}$ in turn. So $-m^{2}$ must be roughly $(100 \mathrm{GeV})^{2} \sim(\mathrm{TeV})^{2}$. Since this is the left-hand side of Eqn.(1.4), $m_{0}^{2}$ and the $\Lambda^{2}$ term must combine to give the correct value for $m^{2}$. But if the Standard Model is a correct description of Nature up to Planckian energies, i.e. $\Lambda \approx 10^{18} \mathrm{GeV}$, then in order to obtain the correct order of magnitude for $m^{2}, m_{0}^{2}$ and the $\Lambda^{2}$ term in Eqn.(1.4) must cancel each other at one part in $10^{32}$ ! In other words, in the absence of this fine tuning of the parameters the "natural" value for $m^{2}$ is $\Lambda^{2}$. Why the electroweak scale of physics is so much smaller than the Planck scale, or any energy scale $\Lambda$ of new physics, is known as the hierarchy problem.

It is the presence of these quadratic divergences that suggest that the Standard Model will be replaced by a more complete theory which will also provide a better understanding of the origin of electroweak symmetry breaking. Since the resulting 
$m^{2}$ is of the right size if $\Lambda \approx \mathrm{TeV}$, new physics in the form of new particles and interactions should be discovered at energies in this range.

Since supersymmetric gauge theories [5], quite remarkably, contain no quadratic divergences ${ }^{1}[6]$, particle physics models based on supersymmetry are promising candidates for resolving the hierarchy problem. In this dissertation, the phenomenology of supersymmetric theories is explored, with attention paid to phenomenological problems with these models. These problems are of the "why are some numbers in these models so small" sort. This is in coherence with the philosophy that argues that the Standard Model is incomplete, e.g. why is $M_{W} / M_{P L} \sim 10^{-16}$ so small?

In supersymmetric field theories all the particles appear in irreducible representations of the supersymmetry algebra, referred to as supermultiplets. Since a supersymmetry generator has a spinorial index, and the members of a supermultiplet are related to each other by supersymmetry transformations, the individual components of a multiplet will not have the same spin. For example, a chiral supermultiplet is

$$
F=\left(\begin{array}{c}
\phi \\
\psi_{\alpha}
\end{array}\right)
$$

and contains a complex scalar $\phi(\operatorname{spin} 0)$ and a two-component fermion $\psi_{\alpha}$ (spin

\footnotetext{
${ }^{1}$ Assuming $\operatorname{Tr} Q_{X}=0$ if there is a $U(1)_{X}$ gauge group.
} 
$1 / 2$ ). Similarly, a vector multiplet is

$$
V=\left(\begin{array}{c}
V_{\mu} \\
\chi_{\alpha}
\end{array}\right)
$$

and contains a massless vector boson $V_{\mu}$ (spin 1) and a two-component fermion $\chi_{\alpha}$. Note that in both cases the number of bosonic and fermionic physical degrees of freedom are equal. Further, the supersymmetry generators commute with the gauge symmetry generators, so that all the components of a supermultiplet have the same gauge group quantum numbers. Finally, a very important point for the cancellation of quadratic divergences is that the supersymmetry generators commute with the spacetime generators. This implies that all the components of a multiplet have the same energy, and in particular, that they all have the same rest mass.

Since supersymmetry transformations interchange bosons and fermions, a supersymmetry transformation does not commute with a Lorentz group transformation. Nonetheless supersymmetry is also a spacetime symmetry since it enlargens the group of spacetime transformations. In fact, the largest possible symmetry of the $S$ matrix ${ }^{2}$ is the product of an internal global or gauge symmetry and a supersymmetry [7]. So supersymmetric theories contain a larger spacetime symmetry than their non-supersymmetric counterparts. This fact suggests that supersymmetry is in some way relevant to Nature.

\footnotetext{
${ }^{2}$ The $S$ matrix gives the quantum mechanical amplitude $\mathcal{A}$ for scattering an initial state into a final state. The probability for this process is then proportional to $|\mathcal{A}|^{2}$.
} 
A heuristic explanation for why supersymmetric theories contain no quadratic divergences is the following. First suppose that the bare mass for the scalar particle in a chiral multiplet is equal to zero. Then since all the components of a supermultiplet have the same mass, the fermion partner $\psi$ is also massless. But in this case the theory contains the chiral symmetry $\psi \rightarrow e^{i \beta} \psi$ for an arbitrary real number $\beta$. This symmetry is sufficient to guarantee that in perturbation theory the fermion is exactly massless. But since by supersymmetry the scalar partner must also receive the same quantum corrections, it therefore remains massless. So there are no quadratic contributions to the mass parameter of the scalar particle.

This is realized in the loop expansion by the cancellation of the quadratic divergences between different Feynman diagrams. In Figure 1.2(a), the scalar boson $\phi_{1}$ receives a quadratic divergence from its $\phi_{1}^{*} \phi_{1} \phi_{2}^{*} \phi_{2}$ interaction with other scalars $\phi_{2}$ (this notation also allows $\phi_{2}=\phi_{1}$ ). There is also a quadratic divergence from its coupling to the fermion superpartner $\psi_{2}$ of $\phi_{2}$. This contribution is shown in Figure 1.2(b) and is numerically exactly the opposite of the first contribution such that the sum of the two Feynman diagram cancels, and there are no quadratically divergent contributions to the mass of $\phi_{1}$.

The argument for the cancellation of the quadratic divergences when the scalar particle is massive is very similar. Supersymmetry implies that the fermion superpartner has the same mass. But the total quantum correction to the fermion mass must be $\Delta m \propto m_{0}$ since in the limit that the bare fermion mass $m_{0} \rightarrow 0$ the chiral symmetry forbids the generation of a mass in perturbation theory. But this cor- 


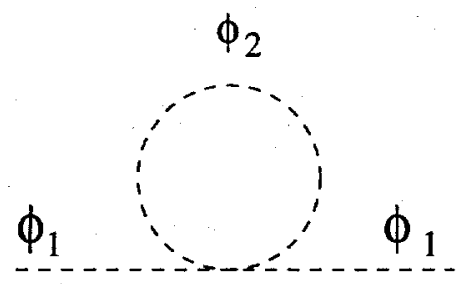

(a)

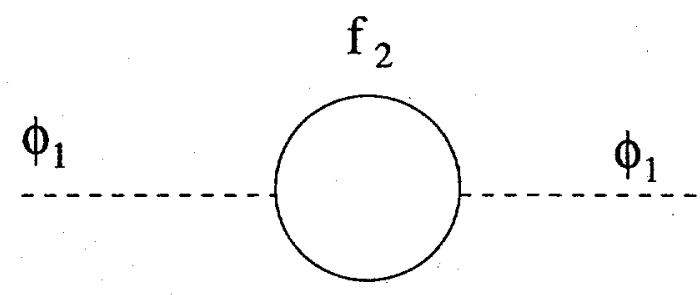

(b)

Figure 1.2: Cancellation in supersymmetric theories of quadratic divergences to the scalar masses. Solid and dotied lines represent propagating fermions and bosons respectively.

rection must by supersymmetry be identical to the correction to the scalar mass. Thus even if the scalar particle is massive it receives no quadratic corrections to its mass.

The absence of quadratic divergences can also be generalized to include supersymmetric gauge theories. In this case, the quadratic divergence from the Feynman diagram with an internal gauge boson is canceled by the quadratic divergence from the Feynman diagram with an internal superpartner of the gauge boson, the gaugino. So in supersymmetric theories there are no quadratic divergences ${ }^{3}$. To re-cap, the basic reason for this is that supersymmetry relates the scalar particle to its fermion superpartner which does not receive any quadratic divergences. This relationship then protects the scalar particle from receiving any quadratic divergences as well.

\footnotetext{
${ }^{3}$ See the previous footnote for the one restriction. Curiously, this condition is also the same as requiring the cancellation of the gravitational-gravitational- $U(1)_{Q}$ anomaly.
} 
So a supersymmetrized version of the Standard Model does not contain any quadratic divergences and the hierarchy problem is at least stabilised. That is, once the huge disparate scales $M_{Z}$ and $M_{P l}$ are initially established, supersymmetry insures that quantum corrections do not push the vev of $H$ (and hence the masses of all the Standard Model particles) up to the Planck scale.

In fact, the Planck scale and the weak scale are probably not the only mass scales in Nature. For example, the scale of Grand Unification (see later), or the mass scale of some new flavor physics, probably exist and are most likely orders of magnitude larger than the weak scale. If scalar particles are in some way involved in the physics at these other mass scales (just as the Higgs particle might be involved in electroweak symmetry breaking), then in a non-supersymmetric theory quantum corrections would tend to bring all these different mass scales together. That is, the separation of mass scales could only be maintained at the expense of an unnatural amount of fine tuning among the bare parameters of the theory.

In supersymmetric theories, in contrast, there are non-renormalization theorems which imply that no fine tuning is required to maintain these different mass scales [6]. These generalize the cancellation of the quadratic divergences discussed before. Similarly, non-perturbative non-renormalization theorems for the dimensionless "Wilsonian" gaugè-couplings and "superpotential" couplings exist and are presented in Chapter 2. So in a supersymmetric theory of Nature it is possible to envision a hierarchy of mass scales which are not destabilized by quantum cor- 
rections. In these theories it is then sensible to address issues such as the physics occurring at, and the possible origins of, these different mass scales.

So what does a supersymmetrized version of the Standard Model look like? The simplest solution is to promote each gauge and matter particle of the Standard Model to a complete supermultiplet. Thus the theory now includes some fermion superpartners (the gauginos) to the gauge bosons, and scalar superpartners to the quarks and leptons, referred to as squarks and sleptons, respectively. So a supersymmetric version of the Standard Model contains at least twice as many particles.

What about the Higgs particle $H$ ? An inspection of Table 1.1 indicates that the leptons $L$ have the same quantum numbers as the Higgs scalar $H^{*}$. This suggests the interesting possibility that the Higgs bosons could be the boson superpartners of the leptons. In this case the Higgs field would have a lepton number, since it would be in the same supermultiplet as the leptons. The breaking of electroweak symmetry would also break lepton number and this has some phenomenological difficulties, such as generating neutrino masses that are too large. A more serious problem though is that it turns out that such a theory (with 3 Higgs doublets) cannot generate masses for the up-type quarks. For this reason this possibility is not considered further in this dissertation.

So instead the Higgs sector is made supersymmetric by introducing fermion superpartners, referred to as 'higgsinos', thus promoting $H$ to a supermultiplet. The higgsinos contribute to the gauge anomalies since they carry charge, and 
in fact the introduction of only one Higgs supermultiplet results in non-vanishing $S U(2)_{L} \times S U(2)_{L} \times Y$ and $Y^{3}$ gauge anomalies. These anomalies must be canceled to preserve the unitarity of the theory. So an additional Higgs multiplet, $\bar{H}$, with the opposite hypercharge, is introduced to cancel the anomalies. Another difficulty with introducing only one Higgs supermultiplet is that supersymmetry prevents $H$ from giving mass to the leptons and down-quarks. It is perhaps surprising that this is not the case for $\bar{H}$. For these two reasons two Higgs doublets are included in the minimal supersymmetric extension to the Standard Model (the MSSM). In this model then the mass for the up-quarks is obtained from the vev of $H$, and that of the leptons and down-quarks from the vev of $\bar{H}$. The matter content of the MSSM is also given in Table 1.1, where now each field $\psi$ is interpreted as a supermultiplet.

The previous arguments establishes the particle content of the MSSM. But what about the interactions between all these particles? These are obtained by including all interactions that are renormalizable and consistent with supersymmetry and all the gauge symmetries.

The requirement that the theory is renormalizable is not necessary, and in fact is not expected. This is because the MSSM, just like the SM, is probably only an effective theory, rather than a fundamental theory of Nature. At energies above some unknown physical mass scale $M$, such as the Grand Unified mass scale, the mass scale of some new flavor physics, or the Planck scale, to suggest a few, the MSSM is replaced by a more fundamental theory. The effect of this high-energy 


\begin{tabular}{||c||c|c|c||c||c|c|c|}
\hline$\psi$ & $S U(3)_{c}$ & $S U(2)_{L}$ & $U(1)_{Y}$ & $\psi$ & $S U(3)_{c}$ & $S U(2)_{L}$ & $U(1)_{Y}$ \\
\hline$Q$ & $\mathbf{3}$ & $\mathbf{2}$ & $1 / 6$ & $L$ & $\mathbf{1}$ & $\mathbf{2}$ & $-1 / 2$ \\
\hline$u^{c}$ & $\overline{\mathbf{3}}$ & $\mathbf{1}$ & $-2 / 3$ & $e^{c}$ & $\mathbf{1}$ & $\mathbf{1}$ & 1 \\
\hline$d^{c}$ & $\overline{\mathbf{3}}$ & $\mathbf{1}$ & $1 / 3$ & $\nu^{c}$ & $\mathbf{1}$ & $\mathbf{1}$ & 0 \\
\hline$H$ & $\mathbf{1}$ & $\mathbf{2}$ & $1 / 2$ & $\bar{H}$ & $\mathbf{1}$ & $\mathbf{2}$ & $-1 / 2$ \\
\hline
\end{tabular}

Table 1.1: Quantum number charges of one generation of Standard Model particles with a right-handed neutrino, and two Higgs fields, under the Standard Model gauge group $G=S U(3)_{c} \times S U(2)_{L} \times U(1)_{Y}$. Here $Q=\left(u_{L}, d_{L}\right), L=\left(\nu_{L}, e_{L}\right)$, $H=\left(h^{+}, h^{0}\right)$, and $\bar{H}=\left(\bar{h}^{0}, h^{-}\right)$. The electric charge of a particle is $Q_{e m}=Y+T_{3 L}$, where here $T_{3 L}$ is the value of the diagonal generator of $S U(2)_{L}$ acting on $\psi$, e.g. $+1 / 2$ for $u_{L}$ and $-1 / 2$ for $d_{L}$. 
physics at low-energies, i.e. $E<<M$, is to either generate interactions suppressed by $M$, or to determine the parameters of the low-energy theory. These nonrenormalizable interactions are important if they violate some global symmetries of the low-energy theory such as baryon number. Otherwise, their effect on physical processes is smaller by roughly $E / M$ compared to the effect of the renormalizable interactions. So they are irrelevant and it is sufficient to study the renormalizable interactions of the low-energy theory.

The most general supersymmetric Standard Model that is consistent with all the gauge symmetries has some phenomenological problems though. These problems involve the stability of the proton, and the mass spectrum of the superpartners. These are discussed in turn.

Recall that the Higgs supermultiplet $\bar{H}$ and the lepton supermultiplets $L_{i}$ have the same gauge quantum numbers. See Table 1.1. This was the origin of the rejected speculation that the Higgs bosons could be the superpartners of the leptons. In this context, though, this means that from any particle interaction that contains $\bar{H}$, a new interaction consistent with all the gauge symmetries and renormalizablity is obtained by replacing $\bar{H} \rightarrow L_{i}$. Since the interactions with $\bar{H}$ conserve lepton number ${ }^{4}$, the new interactions with the replacement must violate

\footnotetext{
${ }^{4}$ The lepton number $L$ of a lepton and anti-lepton is defined to be +1 and -1 respectively. The lepton number for the quarks and Higgs particles is 0 . Similarly, the baryon number $B$ for quarks (anti-quarks) is defined to be $+1 / 3(-1 / 3)$ (so that for example, the proton has $B=1$ ) and zero for all other particles.
} 


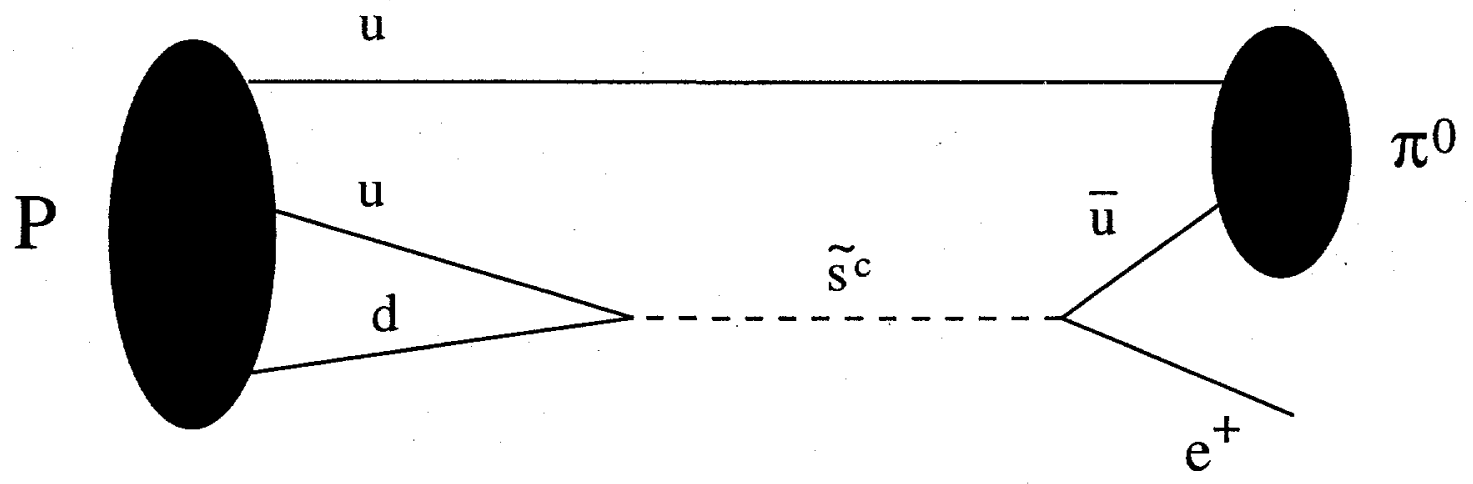

Figure 1.3: $R$-parity violating proton decay $p \rightarrow \pi^{0} e^{+}$.

lepton number.

Further, baryon number violating interactions are also allowed. Trilinear interactions of the form $u^{c} d^{c} d^{c}$ that contain two quarks and one squark are consistent with $S U(3)_{c} \times S U(2)_{L} \times U(1)_{Y}$ invariance. These clearly violate baryon number since the interaction has $B=-1$. Consequently these interactions give rise to processes that change baryon number by one unit.

So the most general supersymmetric extension of the Standard Model, consistent with the principles outlined above, violates lepton and baryon number. On the other hand no lepton or baryon number violating processes, such as $\mu \rightarrow e \gamma$, $\mu \rightarrow$ eee or $n \rightarrow \bar{n}$ oscillation, have been experimentally observed. Thus the reaction rates for these processes must be small if present.

In fact, the presence of both the baryon violating and lepton violating interactions is a disaster. Since both baryon and lepton numbers are no longer conserved the proton is no longer stable. For example, the decay $p \rightarrow \pi^{0} e^{+}$is allowed. If the dimensionless $B$-violating couplings $\lambda_{B}$ and $L$-violating couplings $\lambda_{L}$ which 
characterize the strength of these interactions are numerically close to one, the lifetime for the proton is $10^{-13}-10^{-15}$ seconds. The Feynman diagram for one possible decay mode is given in Figure 1.3. This lifetime is incompatible with the measured lower bound to the proton lifetime of about $10^{32}$ years. It can be made compatible only if the product of the $B$-violating couplings $\lambda_{B}$ and $L$-violating couplings $\lambda_{L}$ are extremely tiny: $\lambda_{B} \lambda_{L}<10^{-25}$.

Why these couplings are so small is a puzzle, and suggests that either $B$ or $L$ is a good symmetry of the renormalizable interactions. These dangerous interactions can be forbidden by imposing a discrete symmetry, called $R$-parity. It is implemented by requiring that the particle interactions are invariant under the discrete symmetry $M \rightarrow-M$ for a matter supermultiplet, and $H_{i} \rightarrow H_{i}$ for each Higgs supermulitplet. This symmetry allows the trilinear interactions of the form $M_{i} M_{j} H_{k}$ which give mass to the matter particles, but forbids interactions of the type $M_{i} M_{j} M_{k}$. It is the latter interactions that make the proton unstable, for example. An inspection of Figure 1.3 indicates that the troublesome vertices contain 3 matter fields, which would be forbidden if this $R$-parity is a good symmetry.

But since the proton decay requires both $B$ number and $L$ number violation, the phenomenological difficulties are not nearly as serious if only one of these numbers is conserved. Thus the imposition of $R$-parity is perhaps too strong, and maybe only $B$ number or $L$ number conservation is sufficient. What are the phenomenological constraints in this case? Since the Yukawa couplings exhibit a hierarchical structure there are good theoretical reasons to expect a hierarchical 
structure for the $L$ number violating interactions if they exist. In Chapter 3 of this dissertation rare processes such as $K^{+} \rightarrow \pi^{+} \nu \bar{\nu}$, as well as top quarks decays, are used to constrain these $L$ number violating interactions.

The next difficulty with a naive supersymmetric extension of the Standard Model occurs with the mass spectrum of the superpartners. As mentioned before, the supersymmetry generators commute with the spacetime translation generators. This implies that all the components of a supermultiplet have the same mass. So for example, if supersymmetry were an exact symmetry of Nature, the superpartner of the electron, the slepton, would have the same mass as the electron. This is incompatible with experimental observations, since no such selectrons have been detected. In fact, the current lower bound on the selectron mass from the LEP2 experiment is about $80 \mathrm{GeV}$, which is $10^{5}$ times heavier than the electron.

These experimental facts imply that supersymmetry must be spontaneously or "softly" broken. Since the motivation for supersymmetry was to solve the hierarchy problem, the spontaneous breaking of supersymmetry must not reintroduce quadratic divergences. Fortunately, the number of types of interactions that break supersymmetry, but do not introduce quadratic divergences is small [8]. Masses for the squarks, sleptons and gauginos may be added without introducing quadratic divergences. These so-called "soft masses" are arbitrary and unrelated to the masses of their superpartners. A very heuristic explanation for why no quadratic divergences are introduced when these soft masses are introduced is the following. First, since no quadratic divergences are present when the boson $m$ and fermion 
$m_{F}$ masses are equal, the one-loop quantum correction to the scalar mass for unequal masses is then

$$
\Delta m^{2}=C\left(m^{2}-m_{F}^{2}\right)+\Delta m_{F}^{2} .
$$

Here $C$ is a function of the gauge and Yukawa couplings of the theory and $\log \Lambda^{2}$, and $\Delta m_{F}^{2}$ is the correction to the fermion mass. This is seen more clearly (at one-loop) by evaluating the Feynman diagrams in Figure 1.2. The diagram in Figure 1.2(a) depends on the scalar mass $m$, but not on the fermion mass $m_{F}$, whereas the opposite is true for Figure 1.2(b). This, together with the fact that $\Delta m^{2}=\Delta m_{F}^{2}$ in the supersymmetric limit $m=m_{F}$, implies that the correction to $\Delta m^{2}$ when $m \neq m_{F}$ must be of the form as in Eqn.(1.7).

So it is possible to give large enough masses to the superpartners of the Standard Model fields so as to avoid experimental detection, while simultaneously not introducing any quadratic divergences.

The existence of these soft masses for the squarks and sleptons also provides a natural explanation for the origin of electroweak symmetry breaking. Recall that in the Standard Model this Higgs mass parameter $m_{H}^{2}$ must be negative in order for electroweak symmetry to be broken. This is introduced into the theory without any explanation. In the MSSM by contrast, a negative $m^{2}$ for the Higgs mass parameter occurs quite naturally, and is made possible by the large top quark Yukawa coupling and for top squark masses heavier than about $100 \mathrm{GeV}$. This is because the quantum corrections of the top squarks to $m_{H}^{2}$ (as in Figure 
1.2(a) with $\phi_{2}=$ top squark) are always negative. On the other hand, the same quantum corrections result in a less negative correction to the squarks masses. The squarks also receive positive corrections from loops of gluinos, which do not contribute to $\Delta m_{H}^{2}$. These tend to keep $m^{2}>0$ for the squarks. A $m^{2}<0$ for a squark mass would be bad since this would break $S U(3)_{c}$, and result in a weakly interacting short range force (like $\left.S U(2)_{L}\right)$ rather than the confining force that is observed. Further, the color quantum numbers would not be conserved. This, however, does not occur - only the Higgs mass parameter becomes negative and the squark and slepton $m^{2}$ s remain positive. Thus the perturbative dynamics of the MSSM with a mass spectrum between $100 \mathrm{GeV}$ and $1 \mathrm{TeV}$ predicts that the electroweak symmetry should be broken, and that the color and electromagnetism symmetries should be unbroken.

The introduction of completely arbitrary soft masses for the squarks and sleptons has some phenomenological problems though. In the Standard Model individual lepton number is conserved. There are no processes such as $\mu \rightarrow e \gamma$, $\tau \rightarrow e \gamma$, or $\mu \rightarrow e e e$ which violate individual lepton number. This follows directly from the symmetry $l_{i} \rightarrow e^{i \beta_{i}} l_{i}$ of the SM interactions, where here $l_{i}$ is a lepton fermion. For generic soft masses in the MSSM this is not the case though. Since there are 3 generations of $X=Q, u^{c}, d^{c}, L$ and $e^{c}$ sparticles, there are in general five $3 \times 3$ arbitrary mass matrices $m_{X}^{2}$, one for each of the fields listed above. To see that these generically break the flavor quantum numbers, first rotate the fermions and their superpartners by a common rotation so that the fermion mass 
matrices are diagonalised. In this basis all the supergauge interactions conserve flavor. The matrices $m_{X}^{2}$, however, will not in general be diagonal in this basis. This means, for example, that separate rotations on $\tilde{e}$ and $\tilde{\mu}$ are not allowed if $m_{\tilde{e} \tilde{\mu}}^{2} \neq 0$. Thus individual "smuon" and "selectron" numbers are not conserved. But since the sleptons have the same global symmetries as the leptons because they are in the same supermultiplet, individual electron and muon numbers are also not conserved. Thus the presence of this mass mixing between smuons and selectrons violates lepton number, and they result in many dangerous lepton violating processes. For example, the Feynman diagram resulting in the decay $\mu \rightarrow e \gamma$ is given in Figure 1.4. Since the branching fraction $B R(\mu \rightarrow e \gamma)<10^{-11}$ is very small, the mass mixing must be extremely small :

$$
\frac{m_{\tilde{e} \tilde{\mu}}^{2}}{m^{2}} \lesssim 10^{-2}\left(\frac{100 \mathrm{GeV}}{m}\right)^{2}
$$

This $m_{\tilde{e} \tilde{\mu}}^{2}$ is related to the mass difference $\Delta m^{2}$ between the two slepton eigenstates and the mixing angle $\sin \theta$ that diagonalises them (in the mass basis where the leptons are diagonal):

$$
m_{\tilde{e} \tilde{\mu}}^{2}=\left(\Delta m^{2}\right) \sin \theta \cos \theta
$$

A similar mass mixing between the strange and down squarks leads to large flavor changing processes, and in particular contributes to $\Delta m_{K}$, the mass difference of the neutral kaons. The Feynman diagram for a possible process is given in Figure 1.5. Since $\Delta m_{K} / m_{K} \approx 10^{-14}$ is measured to be very small, in order to be consistent with this measurement the masses of the first two generations squarks 


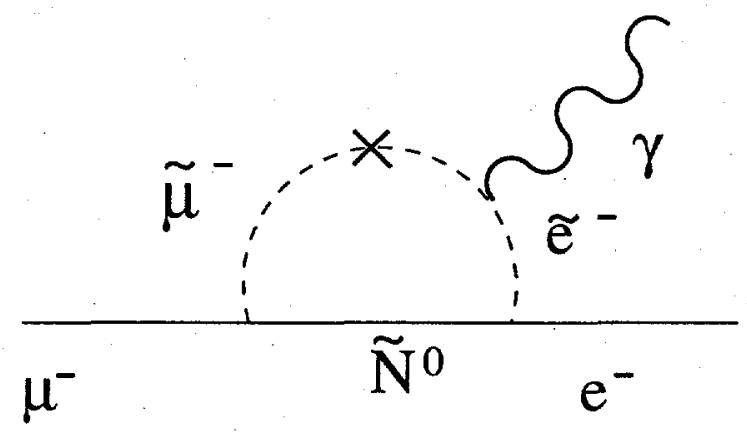

Figure 1.4: A supersymmetric contribution to $\mu \rightarrow e \gamma$. Here $\tilde{\mu}$ is a smuon, $\tilde{e}$ is a selectron, and $\tilde{N}^{0}$ is a neutralino. The "cross" indicates a $\tilde{\mu} \rightarrow \tilde{e}$ transition.

must be even more degenerate:

$$
\frac{\Delta m^{2}}{m^{2}} \sin \theta \cos \theta \lesssim \text { few } \times 10^{-3}\left(\frac{500 \mathrm{GeV}}{m}\right)^{2}
$$

Thus two $500 \mathrm{GeV}$ squarks must be degenerate to within a few hundred MeV! The $C P$-violating parameter $\epsilon$ in the neutral kaon system provides an even stronger constraint: if $\Delta m^{2}$ contains an order one phase the right-hand side of Eqn (1.10) is a factor of 10 smaller.

Why these sleptons and squarks of different flavors must be so closely degenerate in mass, when there is no good reason to expect them to be, is referred to in the literature as the "supersymmetric flavor problem". There are several physics explanations for why the masses or mixing angles appearing in Eqn.(1.9) and in Eqn.(1.10) are "naturally" small :

1. The short-distance theory contains a flavor symmetry. In the limit of unbroken flavor symmetry the squarks and sleptons are degenerate in mass so that the flavor changing processes are completely suppressed [9]. This flavor symmetry 


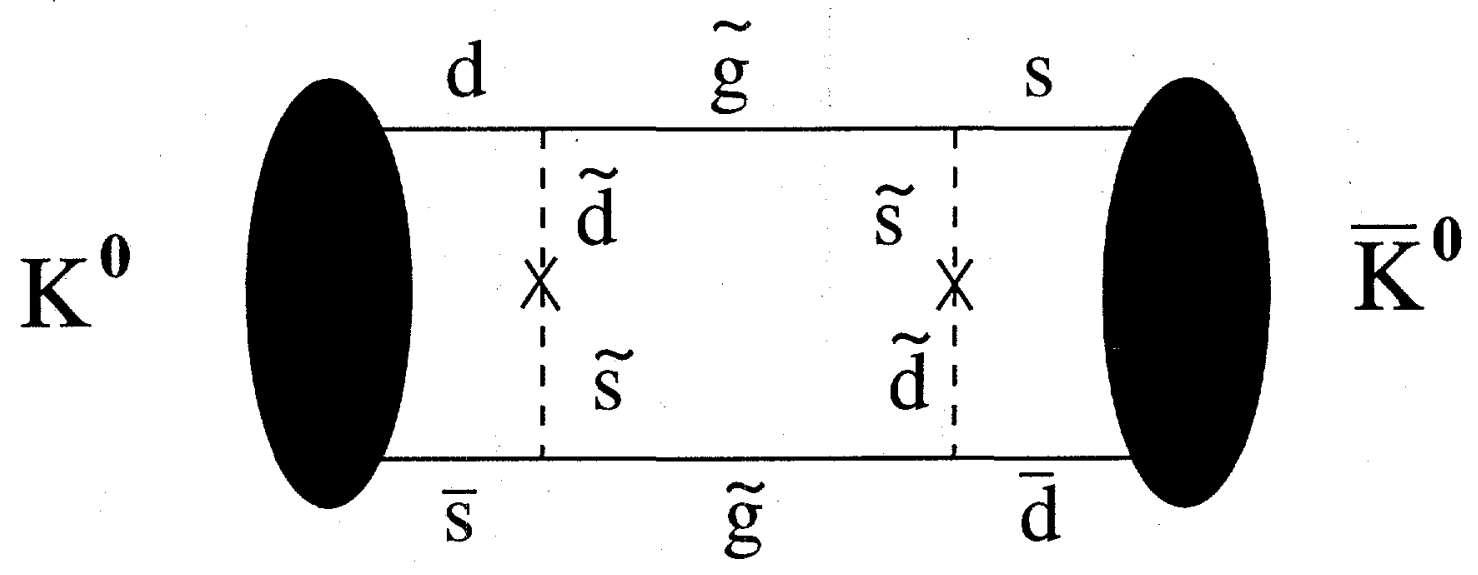

Figure 1.5: A supersymmetric contribution to $K-\bar{K}$ mixing. Here $\tilde{s}$ is a strange squark, $\tilde{d}$ is a down squark, and $\tilde{g}$ is a gluino. The crosses indicate $\tilde{s} \leftrightarrow \tilde{d}$ transitions.

also forbids any of the small Yukawa couplings. For example, in a $U(2)$ theory $[9,10]$, the first and second generation particles form a 2 ; the soft scalar mass interactions must respect this symmetry, so only a common mass $m_{0}^{2}$ for $\tilde{s}$ and $\tilde{d}$ is allowed. The spontaneous breaking of the flavor symmetry is responsible for generating the small Yukawa couplings, and also leads to flavor changing processes as described above which are consistent with the measured values. Since the flavor symmetry is the same for a particle or its superparticle, the hierarchy appearing in $V_{K M}$ for the quarks may also appear in the matrices for the squarks and sleptons. So a large mixing angle between $\tilde{e}$ and $\tilde{\mu}$ is conceivable. In Chapter 4 the prospect for detecting the slepton mass mixing angles at the LHC is considered;

2. The spontaneous breaking of supersymmetry is communicated to the MSSM by the SM gauge interactions at an energy scale below the mass scale responsible for generating the fermion Yukawa interactions [11]. Since the gauge interactions 
do not distinguish between flavors, each slepton flavor receives the same soft mass, and similarly the squark flavors are degenerate. So there are no dangerous flavor changing processes. In Chapter 5 this framework is investigated, and it is found that if the supersymmetry breaking sector is at low-energy, then this solution introduces a large amount of fine tuning of the Higgs mass parameters in order to obtain the correct $Z$ mass. Some ideas for avoiding this difficulty are also presented;

3. The first and second generation scalars are very heavy, $10 \mathrm{TeV}$ or larger so that the flavor changing processes are sufficiently suppressed. In Chapter 6 this idea is investigated, and it is found that in order to avoid breaking color and charge, a fine tuning comparable to that which was required to solve the supersymmetric flavor problem is introduced into the Higgs mass parameters. Thus this solution solves one fine tuning problem but introduces another;

4. The mixing angles are effectively zero. These so-called "alignment" models [12] are not considered in this dissertation.

The next subject discussed here concerns the bizarre representation structure of the Standard Model. Recall that the representations of the Standard Model particles under the Standard Model gauge group are given in Table 1.1. One striking feature of the representations is that they are so different from each other. Where do these hypercharge assignments come from, and why do the particles appear in those particular representations of $S U(3)_{c} \times S U(2)_{L}$ ? Why even $S U(3)_{c} \times S U(2)_{L}$ ? Why not $S U(4) \times S O(7)$ ? Is there a hidden relationship connecting the elements 
of this structure, or is it completely arbitrary? This is reminiscent of Mendeleev's Periodic Table of the Elements and the atomic theory that followed which provided a deeper "picture" of the organization of the elements. Perhaps a more unifying structure is also beneath the Standard Model. As these questions address the structure of the Standard Model, any progress must be found in physics beyond the Standard Model.

To underscore the significance of any possible answers to these questions, a "fake" Standard Model is presented in Table 1.2. In this "fake" Standard Model, the "quarks" occur in the 6 representation of $S U(3)$, rather than the 3 . The gauge anomalies are canceled by having two "leptons" per generation. Note that the $S U(2)$ and hypercharge assignments of the "fake" SM particles are the same as in the SM. So the "fake" SM particles have the same electric charge as their SM counterparts. For one generation of fermions the low-energy physics of the "fake" $\mathrm{SM}$ is qualitatively similar to that of the SM: here $S U(3)_{c}$ probably confines, giving "protons" and "neutrons"; there are also electrons, muons and neutrinos. As in the SM, the Higgs field $H$ can break electroweak symmetry down to $U(1)_{e m}$ and give mass to all the particles. So this "fake" SM imitates many of the qualitative features of the SM, and the difference between the two is in the (important) details. Observers in a Universe with the "fake" SM may also wonder about any underlying unity to their world.

In [13] Georgi and Glashow put forward the beautiful idea that the Standard Model is unified into the single simple gauge group $S U(5)$. An aesthetic 


\begin{tabular}{||c||c|c|c||c||c|c|c||}
\hline$\psi$ & $S U(3)$ & $S U(2)$ & $U(1)_{Y}$ & $\psi$ & $S U(3)$ & $S U(2)$ & $U(1)_{Y}$ \\
\hline$Q$ & $\mathbf{6}$ & $\mathbf{2}$ & $1 / 6$ & $E_{1}$ & $\mathbf{1}$ & $\mathbf{1}$ & 1 \\
\hline$U^{c}$ & $\overline{\mathbf{6}}$ & $\mathbf{1}$ & $-2 / 3$ & $E_{2}$ & $\mathbf{1}$ & $\mathbf{1}$ & 1 \\
\hline$D^{c}$ & $\overline{\mathbf{6}}$ & $\mathbf{1}$ & $1 / 3$ & $H$ & $\mathbf{1}$ & $\mathbf{2}$ & $1 / 2$ \\
\hline$L_{1}$ & $\mathbf{1}$ & $\mathbf{2}$ & $-1 / 2$ & $L_{2}$ & $\mathbf{1}$ & $\mathbf{2}$ & $-1 / 2$ \\
\hline
\end{tabular}

Table 1.2: An anomaly-free "fake" Standard Model. Here $H$ is the Higgs scalar, and all other fields are fermions. The physics of this model (for one generation) is qualitatively similar to that of the Standard Model. But to the author's ability this model cannot be unified into a Grand Unified Theory.

strength of this proposal is that one generation of fermions fill complete $S U(5)$ representations without requiring any additional fermions. Just as remarkable, one generation of fermions, together with a right-handed neutrino $\left(\nu^{c}\right)$, which is independently hypothesized to generate small neutrino masses, together form a complete representation of $S O(10)$, the spinorial representation 16 . The economy of the fermion unification, and the elegance of the unification of the gauge groups into a single gauge group is in itself very compelling.

It is next illustrated how these Grand Unified Theories [14] provide some insight into the origin of the particle content of the Standard Model. In particular, a key feature of these theories is that two unrelated particle groups of the Standard Model, the leptons and quarks, are united into a single representation of the Grand Unified Theory. In other words, the Grand Unified Theories do not distinguish 
between leptons and quarks.

To begin, add to the Standard Model a right-handed neutrino, and introduce an extra $U(1)$, call it $U(1)_{B-L}$. The $B-L$ charge of a quark (anti-quark) is $1 / 3$ $(-1 / 3)$, and the negatively (positively) charged leptons have $B-L$ charge -1 $(+1)$. The $B-L$ charge of the Higgs field is 0 in order for the Yukawa couplings to be $U(1)_{B-L}$ invariant. It is now possible to embed

$$
S U(3)_{c} \times U(1)_{B-L} \in S U(4)
$$

Here $U(1)_{B-L}$ is the third diagonal generator of $S U(4)$. Under this embedding the fundamental of $S U(4)$ decomposes as

$$
4 \rightarrow(3,1 / 3) \oplus(\mathbf{1},-1)
$$

This is just a $q \oplus l$ of the Standard Model! So $\left(\begin{array}{c}Q_{a} \\ L\end{array}\right) \sim 4$ and $\left(\begin{array}{c}Q_{a}^{c} \\ L^{c}\end{array}\right) \sim \overline{4}$. Here $a$ is the $S U(3)_{c}$ index, and the doublets $Q^{c}=\left(\begin{array}{c}d^{c} \\ u^{c}\end{array}\right), L^{c}=\left(\begin{array}{c}e^{c} \\ \nu^{c}\end{array}\right)$ have been introduced. Note that $Q^{c}$ has the opposite baryon number to $Q$. Now what's happened to $U(1)_{Y}$ ? In fact, $Y$ is a linear combination of the $B-L$ in $S U(4)$ and a new $U(1)$, call it $U(1)_{T_{R}}$. The relation between the charges is $Y=(B-L) / 2+T_{R}$. It follows that the $T_{R}$ charges of the $S U(2)_{L}$ doublets $Q, L$ are zero, and $H$ and $\bar{H}$ have charge $1 / 2$ and $-1 / 2$. The $S U(2)_{L}$ singlets $Q^{c}$ and $L^{c}$ have charges $+1 / 2(-1 / 2)$ for the upper (lower) components. That is, $T_{R}$ is just the diagonal generator of an $S U(2)_{R}$, with $Q^{c}, L^{c} \sim 2$. It is then natural to extend the $U(1)_{T_{R}}$ 
to the full $S U(2)_{R}$. Thus the Standard Model particle content can be embedded into the Pati-Salam group $G=S U(4) \times S U(2)_{L} \times S U(2)_{R}[15]$. Other than the right-handed neutrino and the extra Higgs doublet $\bar{H}$, no new matter, i.e. nongauge, particles have been introduced. It is remarkable that the Standard Model fields transform so simply under this new group :

$$
\begin{aligned}
\text { one generation }+ \text { right-handed neutrino } & =(4,2,1) \oplus(\overline{4}, 1,2), \\
\text { two Higgs fields } & =(2,2) .
\end{aligned}
$$

Compare the economy of this particle content to that of the Standard Model given in Table 1.1.

One interesting fact about this semi-unification is that the proton is stable. To see this, first note that the $S U(4)$ gauge bosons decompose as $\mathbf{1 5}=(\mathbf{3}, 4 / 3) \oplus$ $(\overline{3},-4 / 3) \oplus(\mathbf{8}, 0) \oplus(\mathbf{1}, 0)$ under $S U(3)_{c} \times U(1)_{B-L}$. Only the $X \sim(3,4 / 3)$ and $Y \sim(\overline{3},-4 / 3)$ states carry baryon number and can potentially mediate proton decay. The point is that the Standard Model fields are contained in $(4,2,1)$ or $(\overline{4}, 1,2)$, and each of these contains only one $S U(3)_{c}$ representation, i.e. $Q$ or $Q^{c}$, but not both. Consequently, the four-Fermi operator obtained by integrating out the massive $S U(4)$ gauge bosons $X$ or $Y$ always contains two leptons, and never three quarks. So the four-Fermi operator conserves lepton number and the proton is stable. As will be seen later, this is in contrast to the predictions of more popular Grand Unified Theories.

The embedding of the Standard Model into the Pati-Salam gauge group re- 
sults in an economy of particle content, but not of the gauge groups since there are still three gauge group factors. A further unification into $S O(10)$ or $S U(5)$ is possible though. The isomorphisms $S U(4) \cong S O(6)$ and $S O(4) \cong S U(2) \times S U(2)$ imply that the Pati-Salam gauge group is isomorphic to $S O(6) \times S O(4)$ which is a maximal subgroup of $S O(10)$. This line of thought suggests that it is natural to embed the Pati-Salam gauge group into $S O(10)$. This is in fact possible with an even further increase of economy. The spinorial representation of $S O(6)$ is 8 and is reducible to $4 \oplus 4^{\prime}$ due to chirality. Under the $S O(6) \cong S U(4)$ isomorphism these spinorial representations get mapped to the fundamental and anti-fundamental representations of $S U(4)$. Likewise, under the second isomorphism the spinorial representation of $S O(4), 4 \rightarrow(1,2) \oplus(2,1)$. Thus using this isomorphism $(\mathbf{4}, \mathbf{2}, \mathbf{1}) \oplus(\overline{4}, \mathbf{1}, \mathbf{2}) \rightarrow(\mathbf{4} \otimes \mathbf{2}) \oplus(\mathbf{4} \otimes \mathbf{2})$ under $S O(6) \times S O(4)$. But this is just the decomposition of the $\mathbf{1 6}$ of $S O(10) \rightarrow S O(6) \times S O(4)$. The conclusion is that a single generation of the Standard Model, plus a right-handed neutrino, fits exactly into the $\mathbf{1 6}$ representation of $S O(10)$.

This unification is very nice, but is it a generic feature of low-energy particle physics models, or is it more unique? That is, is it likely than an arbitrary lowenergy particle physics theory can be embedded into a Grand Unified Theory? The answer is most likely "no", but the author has no proof. Instead, the "fake" SM is presented as an example of a low-energy physics theory that is qualitatively similar to the SM, but most likely cannot be unified into a Grand Unified Theory. While the unification of the Standard Model into $S O(10)$, or similarly, $S U(5)$, 
is aesthetically pleasing and probably difficult to achieve in other "fake" Standard Models, is there any other reason to believe that a Grand Unified Theory exists? In fact, the simple Grand Unified Theories have some interesting phenomenological implications, such as the instability of the proton and the unification of the gauge couplings.

It was stated earlier that the Grand Unified Theories do not distinguish between quarks and leptons. Experimentally, however, this is clearly not the case: quarks confine into protons and neutrons, leptons do not; quark masses are different from lepton masses; quark and lepton interactions are not the same, for example, the cross-sections $\sigma\left(\bar{d}_{R} \bar{d}_{R} \rightarrow \bar{d}_{R} \bar{d}_{R}\right)(E)$ and $\sigma(L L \rightarrow L L)(E)$ (here $E$ is a typical energy scale appearing in the interaction) are different. How can this be reconciled with a Grand Unified Theory that unites quarks and leptons?

In Grand Unified Theories there are many massless gauge bosons beyond those of the Standard Model. For example, there are the $X$ and $Y$ bosons in the PatiSalam group, there are twelve extra gauge bosons in $S U(5)$, and thirty-three in $S O(10)$. Since they have not been detected yet, these gauge bosons must be massive, and this means that the Grand Unified Theory must be spontaneously broken at some mass scale $M_{G U T}$ which is representative of the masses of the extra gauge bosons. There is a decoupling theorem [16] which states that since these gauge bosons are heavy and their masses do not break any of the SM gauge symmetries, they have no measurable effect on scattering experiments performed at energies $E<<M_{G U T}$ and their only effect is to renormalize the parameters of 
the low-energy theory ${ }^{5}$. Thus, except for phenomena forbidden in the Standard Model such as proton decay, the physics of a Grand Unified Theory does not appear in any of the low-energy experiments. So at energies $E<<M_{G U T}$ the effective theory is the SM (or MSSM), rather than a Grand Unified Theory. This is the origin of the differences between leptons and quarks listed earlier: they are caused by physics in the low-energy theory. What this does mean, however, is that only at energies $E \gtrsim M_{G U T}$ are the symmetries of the Grand Unified apparent. For example, in the minimal $S U(5)$ theory both $d^{c}$ and $L$ are unified together into a single $\overline{5}$. Thus at these high energies, for example, the cross-section $\sigma\left(\bar{d}_{R} \bar{d}_{R} \rightarrow \bar{d}_{R} \bar{d}_{R}\right)\left(E \gtrsim M_{G U T}\right)=\sigma(L L \rightarrow L L)\left(E \gtrsim M_{G U T}\right)$, and the quarks and leptons are not distinguishable.

It is interesting to see how this last result comes about. At low energies the cross-sections are different because the gauge couplings of the Standard Model, $\alpha_{1}$, $\alpha_{2}$ and $\alpha_{3}$ are all different. These couplings are measured at LEP using electron beams with energy $E \approx M_{Z} \approx 100 \mathrm{GeV}$. As the energy of a physical process is increased, however, virtual quantum effects of order $\left(\log E / M_{Z}\right)$ become large and their effects can be summed up into an effective coupling $\alpha_{i}(E)$. Since in a simple Grand Unified Theory there is only one gauge coupling $\alpha_{G U T}$, at energies $E \gtrsim M_{G U T}$, the couplings $\alpha_{i}(E)$ of the Standard Model should all become equal

\footnotetext{
${ }^{5}$ Unless the experiment is searching for a process that is forbidden by the symmetries of the low-energy theory, but allowed by the interactions of the high-energy theory, e. g. proton decay. Then the high-energy physics is the leading effect.
} 
to the one gauge coupling $\alpha_{G U T}(E)$.

So the point is that a necessary condition for simple Grand Unified Theories is that the Standard Model couplings should become equal (unify) at energies $E \approx M_{G U T}$. Further, the energy-dependence of the gauge couplings depends on the particle content of the effective theory. Thus the gauge couplings of the Standard Model gauge groups may or may not unify with just the Standard Model particle content, or the particle content of some simple extension. It depends on both the particle content and, of course, the experimentally measured values of the Standard Model gauge couplings.

So do the couplings of the Standard Model or its simple extensions unify at high energies? In fact, as is evident from Figure 1.6, the unification is not very good in the Standard Model. In contrast, the gauge couplings in the MSSM do unify at the few percent level, which is well-within theoretical uncertainties such as the sparticle and Grand Unified mass spectrum. This is presented in Figure 1.7. This is remarkable since the particle content of the MSSM is dictated by the requirement of supersymmetry and the cancellation of gauge anomalies, and not of unification. Since this unification is highly non-trivial, it is a strong piece of indirect evidence for both low-energy supersymmetry and supersymmetric Grand Unified Theories.

Another nice consequence of a supersymmetric $S O(10)$ GUT is that it provides some theoretical explanation for why $R$-parity is a good symmetry. Recall that the dangerous $R$-parity violating interactions are of the form $M_{i} M_{j} M_{k}$, where $i, j$ 


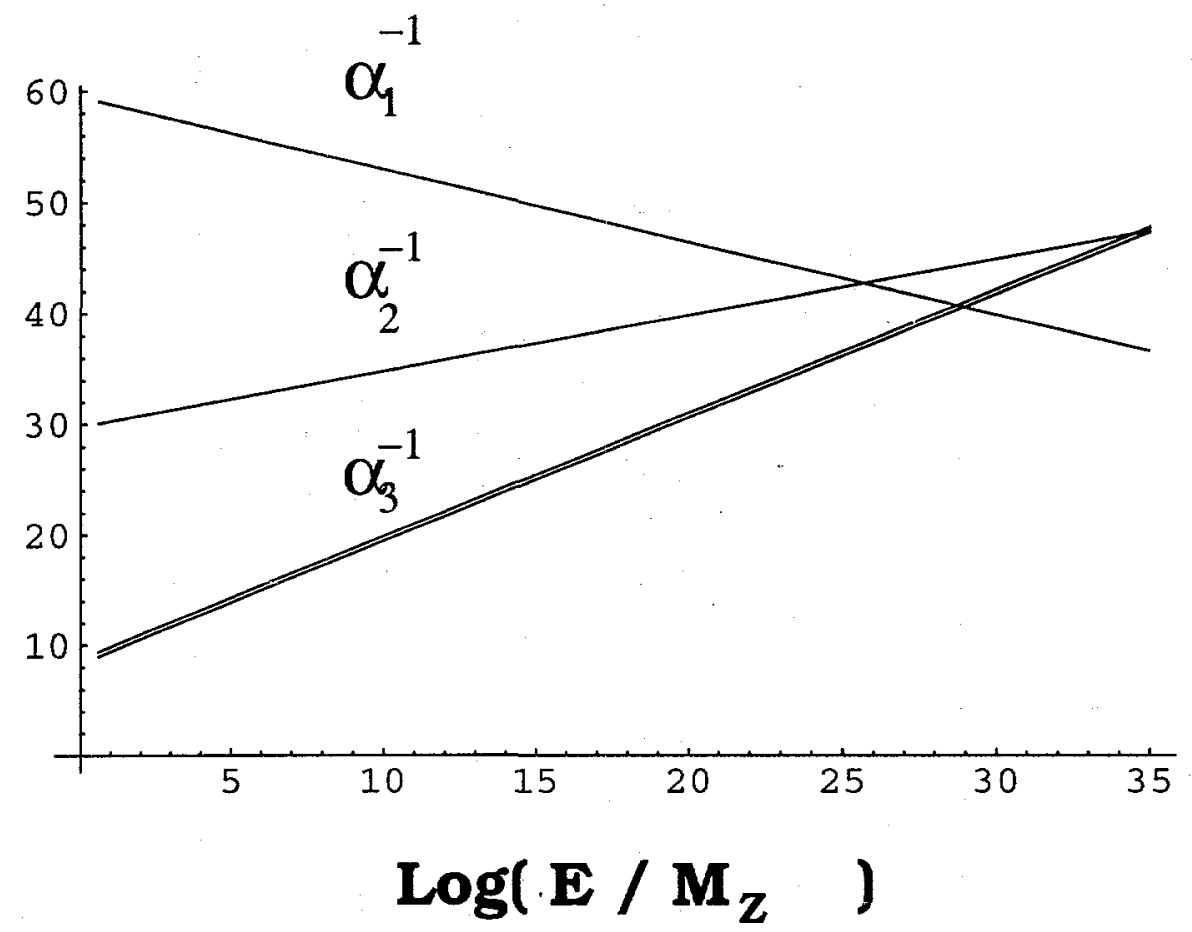

Figure 1.6: Scaling of gauge couplings in the Standard Model. The band corresponds to the $1 \sigma$ error $(0.003)$ on $\alpha_{3}\left(M_{Z}\right)$. The couplings do not appear to unify. 


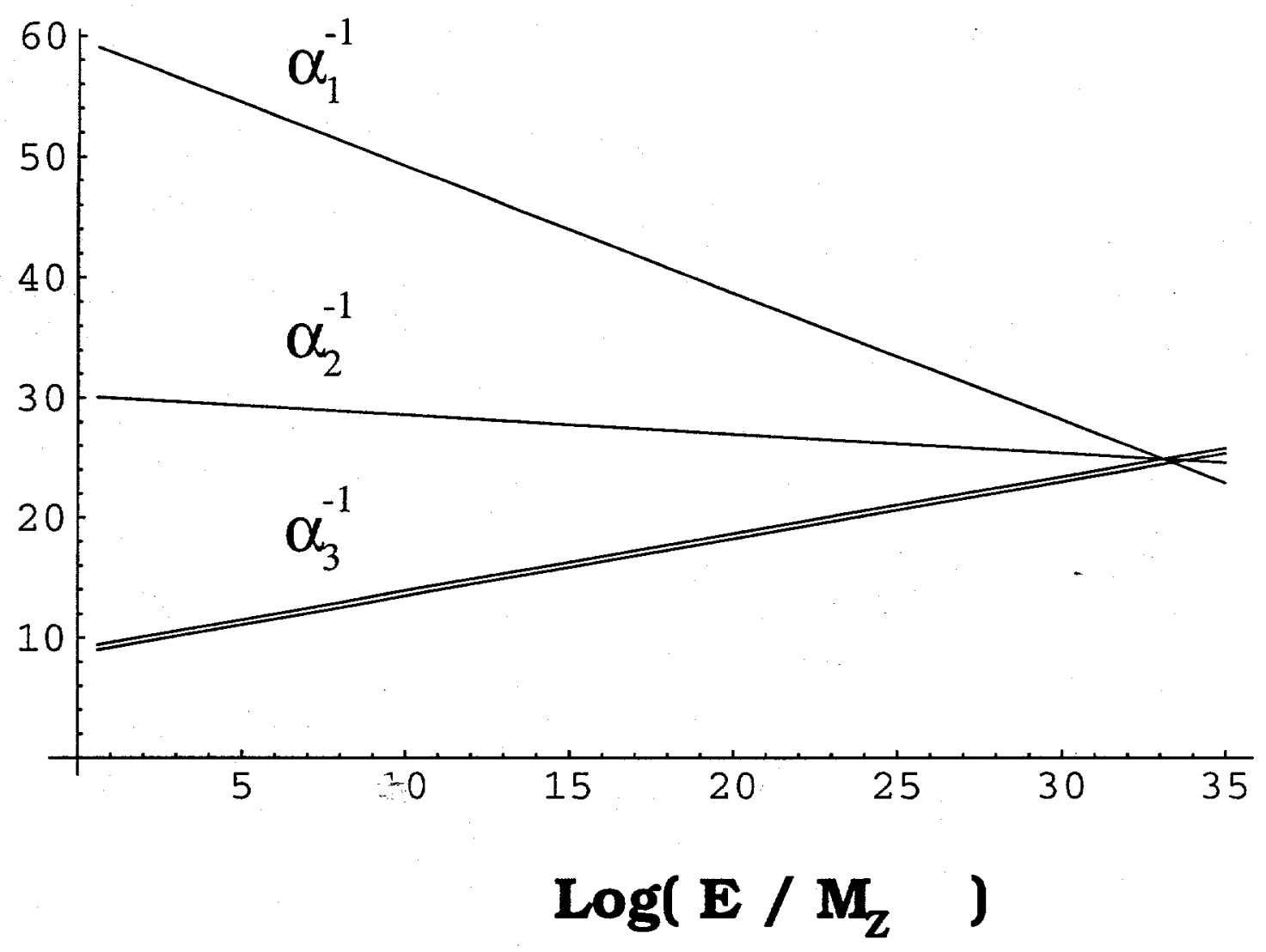

Figure 1.7: Scaling of the gauge couplings in the Minimal Supersymmetric Standard Model. The band corresponds to the $1 \sigma$ error $(0.003)$ on $\alpha_{3}\left(M_{Z}\right)$. The couplings do appear to unify.

and $k$ are flavor labels. In $S O(10)$ gauge theories these interactions are forbidden at the renormalizable level. This is because the matter fields are in the $\mathbf{1 6}$, so the dangerous operator must be of the form $\mathbf{1 6}_{i} \mathbf{1 6} \mathbf{6}_{j} \mathbf{1 6}$. These interactions, however, are not $S O(10)$ gauge invariant and so they do not exist.

A further inspection of Figure 1.7 indicates that the gauge couplings unify at a mass scale $M_{G U T} \approx 2 \times 10^{16} \mathrm{GeV}$. This is somewhat puzzling for the following reason. The only "fundamental" mass scale in Nature is the Planck scale, 
$M_{P L} \approx 10^{18} \mathrm{GeV}$ obtained from Newton's constant. It then seems unlikely that the inferred unification scale $M_{G U T}$ is a fundamental scale in Nature.

The philosophy assumed in this dissertation is that the small number $M_{G U T} / M_{P l} \approx 10^{-2}$ is not fundamental, but rather hints at some more underlying physics. This is explored in Chapter 7 where two candidate Grand Unification models are proposed, with Grand Unified gauge groups $S U(6)$ and $S O(10)$, which generate the Grand Unification from the Planck Scale, i.e., the unification mass scale $M_{G U T}$ is not introduced into the theory. This is roughly achieved through the supersymmetric analog of dimensional transmutation, whereby the small coupling of a gauge group generates a mass scale at low-energies through some non-perturbative dynamics.

In addition, the models presented in Chapter 7 - in particular, the $S O(10)$ model - also maintain the spirit of "unification and simplification". In Grand Unified models the gauge group must be broken down to the Standard Model gauge group. This is achieved by the Higgs mechanism, just as in the Standard Model, by introducing some scalar particles transforming under some representation of the Grand Unified gauge group. For example, in minimal $S U(5)$ a 24 is introduced, whereas in phenomenologically successful $S O(10)$ models many 45 s and $\mathbf{5 4 s}$ must be introduced. In the end, many particles must be introduced and the symmetry breaking must be introduced into the theory. This is clearly the ugly part of these models. In contrast, in the $S O(10)$ model of Chapter 6 , the representation structure is rather simple. Further, the symmetry breaking is not introduced into 
the theory. The correct vacuum is one of several discrete vacua, and the reason why the correct vacuum was selected depends on the early history the Universe.

Finally, the $S O(10)$ model also contains an unexpected result, and this has to do with the lifetime of the proton. In simple Grand Unified Models, such as $S O(10)$ or $S U(5)$, the heavy gauge bosons can mediate proton decay. It occurs in these models since both $Q$ and $u^{c}$ are unified into a single representation - the 10 in $S U(5)$, and the 16 in $S O(10)$. Thus there is an interaction $\sim X Q u^{c}$. between $X$ and the 10, and also an interaction $\sim X^{*} L d^{c}$ between $X$ and the $\overline{\mathbf{5}}$. The exchange of the $X$ gauge boson causes the proton to decay. In supersymmetric Grand Unified Theories the lifetime of the proton from this process is around $10^{34}-10^{36}$ years and is beyond the reach of existing experiments.

In supersymmetric theories there is also an analogous and more dangerous process in the Higgs sector that causes the proton to decay. In gauge theories with the $S U(5)$ subgroups, the particles must come in complete $S U(5)$ representations. This was automatically satisfied for the fermions and their supersymmetric partners. Recall that in supersymmetric theories there are two Higgs doublets and they do not form complete $S U(5)$ (or of any larger gauge group) representations. If $H \rightarrow \mathbf{5}$ in the $S U(5)$ theory, then there are some "missing $S U(5)$ partners", $H(3)$, with quantum numbers $(3,1,-1 / 3)$. These particle together with the missing partners of $\bar{H}$ may form a Dirac particle and have some arbitrary mass $M_{H_{c}}$.

Since these fields carry charge they affect the evolution of the gauge couplings. Requiring that the gauge couplings still unify (as they appear to) implies that the 


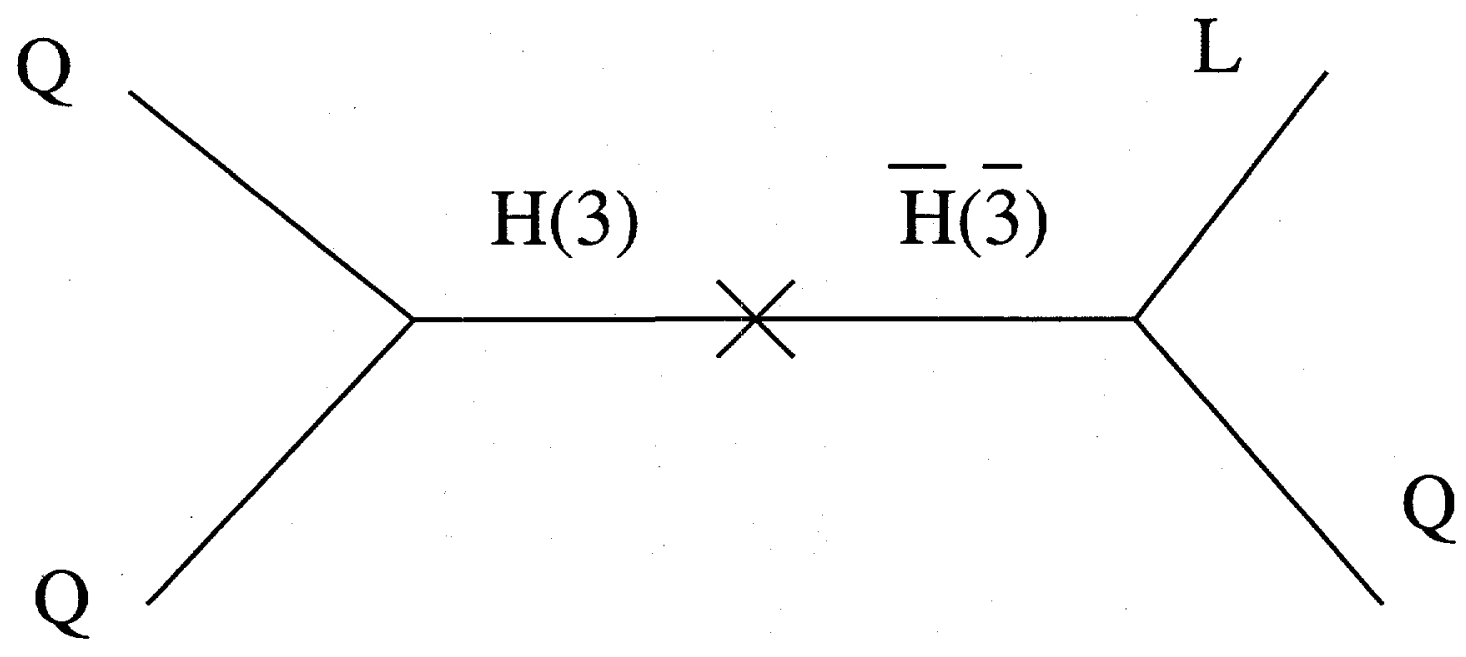

Figure 1.8: Superfield Feynman diagram from colored higgsino exchange leading to proton decay. The "cross" indicates that there is some mass mixing between the two colored higgsinos.

masses of these colored Higgs fields must be close to $M_{G U T}$. Why the masses of the doublets and triplets in the $\mathbf{5}$ and $\overline{5}$ Higgs representation are so wildly different is known in the literature as the "doublet-triplet" splitting problem. In fact, it is possible to prove that the measured couplings unify in supersymmetric theories only if there is a split $S U(5)$ representation, as occurs in the MSSM. (If all the matter (non-gauge) fields formed complete $S U(5)$ representations - i.e. imagine there were no Higgs fields - then $\alpha_{3}\left(M_{Z}\right)=0.07$ is predicted in disagreement with its measured value $0.118 \pm 0.003$.)

These colored Higgs fields can mediate proton decay in $S O(10)$ or $S U(5)$ theories. The Feynman diagram is given in Figure 1.8. The "cross" indicates that there is some mass mixing between $H(\mathbf{3})$ and $\bar{H}(\overline{\mathbf{3}})$. The exchange of the $H(\mathbf{3})$ 
gives a decay $p \rightarrow K^{+} \bar{\nu}_{\mu}$ and $n \rightarrow K^{0} \bar{\nu}_{\mu}$ with a lifetime that is roughly

$$
\tau \sim 10^{29}\left(\frac{M_{H_{c}}}{10^{16} \mathrm{GeV}}\right)^{2} \text { years. }
$$

This is to be compared with the measured lifetime which is larger than $10^{32}$ years. The theoretical and experimental results are naively consistent only if $M_{H_{c}}$ is pushed up to $3 \times 10^{17} \mathrm{GeV}$. This solution is theoretically unattractive as it requires the positing of yet another mass scale.

While there are several solutions to this problem, this dilemma has an unexpected and novel resolution in the $S O(10)$ model of Chapter 7 . Roughly speaking, the particular mechanism that generates the Grand Unification scale also naturally generates tiny couplings of the order $M_{G U T} / M_{P l}$. These tiny couplings naturally appear in the mass mixing of the colored Higgs fields, and result in a suppression of roughly $M_{G U T} / M_{P l}$ in the "cross" appearing in the Feynman diagram in Figure 1.8. This was not introduced into the theory, but was a consequence of the non-renormalizable operators used to split the doublets and triplets. The suppression of the "cross" results in a suppression of roughly $\left(M_{G U T} / M_{P l}\right)^{2} \sim 10^{-3}$ in the decay rate, or in a lifetime of the proton that is a factor of $10^{3}$ larger than before. The resulting lifetime is then consistent with its measured value. 


\section{Chapter 2}

\section{Non-renormalization Theorem for the Wilsonian}

\section{Gauge Couplings in Supersymmetric Theories}

A direct proof that the holomorphic Wilsonian beta-function of a renormalizable asymptotically-free supersymmetric gauge theory with an arbitrary semisimple gauge group, matter content, and renormalizable superpotential is exhausted at 1-loop with no higher loops and no non-perturbative contributions is presented. This is a non-perturbative extension of the well-known result of Shifman and Vainshtein.

\section{$2.1 \quad$ Introduction}

In their 1986 paper [17] Shifman and Vainshtein solved the anomaly puzzle in supersymmetric gauge theories. They argued that the supersymmetric extension of the anomaly equation should be written in operator form and then showed that the coefficient of the trace anomaly involves the Wilsonian gauge beta-function rather than the exact Gell-Mann and Low function [18]. The puzzle is resolved if it can be showed that the Wilsonian gauge beta-function is one-loop exact. A perturbative proof of the above statement was presented in [17] where it was 
argued that all possible operators that could, in principle, contribute to the gauge beta-function beyond one-loop are necessarily of infrared origin, and should not appear in the Wilsonian effective action.

In this Chapter a direct proof that there are no further non-perturbative violations is presented. More specifically, it is proven that the holomorphic Wilsonian beta-function of an arbitrary renormalizable asymptotically-free supersymmetric gauge theory with matter is exhausted at 1-loop with no higher loops and no non-perturbative contributions.

The technique used to employ the theorem was introduced by Seiberg [19] and it is briefly reviewed here. To obtain the beta-function two versions of the theory with different cutoffs and coupling constants and the same low energy physics are compared. The couplings of the theory with the lower cutoff can be expressed in terms of the couplings of the theory with the higher cutoff and the ratio of the two cutoffs. Their functional dependence on the high cutoff couplings is restricted using holomorphy of the superpotential and gauge kinetic terms and selection rules. Holomorphy is a consequence of supersymmetry. To see this, elevate the couplings to background chiral superfields. They must appear holomorphically in the superpotential in order to preserve supersymmetry. Selection rules generalize global symmetries in the sense that the couplings in the superpotential are allowed to transform under these symmetries. Non-zero vacuum values of these couplings then spontaneously break these symmetries. Here only consider $U(1)$ and $U(1)_{R}$ symmetries are considered. In the quantum theory they are generally anomalous, 
but the same technique used for the coupling in the superpotential can be used. That is, it can be assumed that the $\theta$-angle is a background field that transforms non-linearly to make the full quantum effective action invariant.

Then, following a method used in [20], these conditions on the functional relations between the couplings of the theories at different cutoffs are translated into restrictions of the functional form of the gauge beta-function. It can be shown that the gauge beta-function is a function of the holomorphic invariants allowed by selection rules. Then the functional dependence of the beta-function is restricted further by varying the couplings while keeping the invariants fixed. This relates the beta-function of the original theory to the beta-function of a theory with vanishing superpotential. In addition, a strong restriction of the functional dependence of the beta-function on the gauge coupling is obtained. It has exactly the form of a one-loop beta-function. The only ambiguity left is a numerical coefficient which can be calculated in perturbation theory.

Next a short detour is made to explain what is meant by the Wilsonian betafunction [21]. The Wilsonian beta-function describes the renormalization group flow of the bare couplings of the theory so that the low energy theory is cutoff invariant. Additionally, the vector and chiral superfields are not renormalized, i.e. canonical normalization of the kinetic terms [17] is not imposed. The usual convention in particle physics is to canonically normalize the kinetic term. It is obtained by using the covariant derivative $\partial+i g A$. Instead, here non-canonical normalization of the kinetic term is allowed. The normalization of the gauge fields 
is such that the covariant derivative has the form $\partial+i A$. The gauge coupling only appears in front of the gauge kinetic term. In this case it is convenient to combine the $\theta$-angle and gauge coupling constant $g$ into the complex variable $\tau=\theta / 2 \pi+$ $4 \pi i / g^{2}$. In supersymmetric gauge theories the beta-function is holomorphic in the bare couplings only if the fields are not renormalized. Even if there is a canonical normalization at a higher cutoff, the Kähler potential will not be canonical at the lower cutoff. The rescaling of the chiral or gauge superfields is an anomalous transformation [17] that destroys the holomorphy of the superpotential and the beta-function ${ }^{1}$. The relation between the beta-functions in the two normalizations was first discussed in [17]. The beta-function for canonically normalized fields is known exactly [18] and receives contributions to all orders in perturbation theory. For a recent discussion of these issues see also [20]. Again it is emphasized that this Chapter is concerned only with the holomorphic Wilsonian beta-function.

It should also be clearly stated that the proof is not valid if any one of the one-loop gauge beta-functions is not asymptotically-free. This includes the case when the one-loop beta-function vanishes. As will be seen, exactly in this case the $U(1)_{R}$ symmetry is non-anomalous. This makes it difficult to control the dependence of the beta-function on the gauge coupling.

\footnotetext{
${ }^{1}$ For some special theories like $N=2$ SUSY Yang-Mills the rescaling anomaly of the chiral superfields cancels the rescaling anomaly of the vector superfield [20]. For these theories the statements made here are stronger since the canonical and holomorphic Wilsonian couplings coincide.
} 
Various partial versions of this result already existed. As already mentioned, the perturbative non-renormalization theorem was proven in [17]. An analysis of possible non-perturbative violations to this theorem in the case of a simple gauge group with a vanishing superpotential could be found in [20]. It was also known that in the case of a simple gauge group with only Yukawa interactions present in the superpotential, possible non-perturbative corrections to the Wilsonian beta-function are independent of the gauge coupling [22]. It should also be mentioned that for some supersymmetric gauge theories it is possible to determine the exact beta-function for the canonically normalized fields, including all the non-perturbative terms [23]. The exact Wilsonian beta-function for these theories could then be obtained if the rescaling anomaly relating the different normalizations were known exactly, both perturbatively and non-perturbatively. While the rescaling anomaly is used in various places in the literature $[17,23]$ to relate the two gauge couplings, it was not clear to the author whether for these theories the exact form of the anomaly, including non-perturbative terms is known. A perturbative calculation of the anomaly was presented in [20].

Finally, it is noted that the theorem is valid in theories where no mass terms are allowed by the symmetries of the theory. This is of phenomenological interest as many supersymmetric extensions of the Standard Model share this characteristic.

\subsection{Simple Gauge Group}

The case of a simple gauge group $G$ is considered first. Let the generalized 
superpotential $\widetilde{W}$ be defined to include the kinetic term for the gauge fields

$$
\widetilde{W}=\frac{\tau}{64 \pi i t_{R}} \operatorname{tr}_{R}\left(W_{\alpha} W^{\alpha}\right)+W
$$

where

$$
W=\sum \lambda_{i j k} \Phi_{i} \Phi_{j} \Phi_{k}+M \sum m_{i j} \Phi_{i} \Phi_{j}+M^{2} \sum c_{i} \Phi_{i}
$$

is the usual superpotential and $\operatorname{tr}_{R} T^{a} T^{b}=t_{R} \delta^{a b}$. Here $M$ is the cutoff mass and was factored out so that all the couplings are dimensionless. The gauge coupling $g$ and $\theta$-angle are combined in the complex variable

$$
\tau \equiv \frac{\theta}{2 \pi}+\frac{4 \pi i}{g^{2}}
$$

Note that unitarity requires $\tau$ to be valued in the upper half plane. Since $\theta$ is a periodic variable it is convenient to introduce a new variable $q \equiv e^{2 \pi i \tau}$. It is valued in the complex plane and transforms linearly under the anomalous transformations to be discussed below. Weak coupling is at $q=0$.

Consider now a theory with a different cutoff $M^{\prime}$ and with the same low energy physics. The Lagrangian at the new cutoff is

$$
\mathcal{L}=\sum_{i} \int d^{2} \theta d^{2} \bar{\theta} Z_{i} \Phi_{i}^{\dagger} e^{2 V_{h}} \Phi_{i}+\left(\int d^{2} \theta \widetilde{W}\left(\tau^{\prime}, \lambda_{i j k}^{\prime}, m_{i j}^{\prime}, c_{i}^{\prime}, M^{\prime}\right)+\text { h.c. }\right)
$$

where in particular, the fields $\Phi_{i}$ are not renormalized to canonical normalization. The $Z_{i}$ depends non-holomorphically on the couplings, so renormalizing the chiral superfields would destroy the holomorphic form of $\widetilde{W}$. The new coupling $\tau^{\prime}$ is a function of the old dimensionless couplings and the ratio $M / M^{\prime}$. For later 
convenience this is written as

$$
\tau^{\prime}=\tau^{\prime}\left(\tau, \lambda_{i j k}, m_{i j}, c_{i} ; \ln \left(M / M^{\prime}\right)\right)
$$

Supersymmetry requires a holomorphic dependence of $\tau$ on the first four arguments. To see this, note that the couplings in the generalized superpotential can be considered as vacuum values of background chiral superfields. Invariance of the action under supersymmetry transformations requires holomorphy of the superpotential.

To prove the non-renormalization theorem selection rules are used. These are global symmetries of the superpotential with all couplings considered as chiral superfields. The couplings are assigned non-trivial transformation properties under the symmetry group. These symmetries will be spontaneously broken by non-zero vacuum values of the couplings. In general the symmetries are also anomalous. They are are made non-anomalous by assigning a charge to $q$, i.e. transforming $\theta$ to compensate for the anomaly. Consider the $U(1)_{R} \times U(1)$ global symmetry with the following charge assignment:

\begin{tabular}{|c||c|c|c|c|c|c|}
\hline & $W_{\alpha}$ & $\Phi_{i}$ & $\lambda_{i j k}$ & $m_{i j}$ & $c_{i}$ & $q$ \\
\hline$U(1)_{R}$ & 1 & $2 / 3$ & 0 & $2 / 3$ & $4 / 3$ & $2 b_{0} / 3$ \\
\hline$U(1)$ & 0 & 1 & -3 & -2 & -1 & $2 \sum_{i} t\left(R_{i}\right)$ \\
\hline
\end{tabular}

The quantity $b_{0}$ is given by $b_{0}=3 t_{a d j}-\sum_{i} t\left(R_{i}\right)$, where $t\left(R_{i}\right)$ is the normalization of the generators for the representation of the chiral superfield $\Phi_{i}$. For example, 
$t=1 / 2$ for a fundamental of $S U(N)$. Define the gauge $\beta$-function by

$$
\beta_{2 \pi i \tau}=\left.\frac{d}{d \ln \left(M / M^{\prime}\right)} 2 \pi i \tau^{\prime}\right|_{M^{\prime}=M}=\beta\left(\tau, \lambda_{i j k}, m_{i j}, c_{i}\right) .
$$

The holomorphy of $\tau$ in (2.5) translates into holomorphy of the $\beta$-function.

Since $\tau \rightarrow \tau+1$ is a symmetry of the theory, $\beta$ is a single valued function of $q$

$$
\beta_{2 \pi i \tau}=f\left(q, \lambda_{i j k}, m_{i j}, c_{i}\right)
$$

First, consider the case when at least one mass term, call it $m_{*}$, can be non-zero. If any $c_{i}$ could be non-zero, then there is a gauge singlet field which could be given a Majorana mass, so this is the same case as above.

The gauge beta-function is $U(1)_{R} \times U(1)$ invariant. This statement is nontrivial and requires some explanation. Consider some arbitrary coupling $\lambda$ that transforms linearly under some $U(1)$ or $U(1)_{R}$ symmetry. Its beta-function $\beta_{\lambda}$ must also transform linearly with the same charge as $\lambda$

$$
e^{i Q_{\lambda} \alpha} \beta_{\lambda}(\lambda, \ldots)=\beta_{\lambda}\left(e^{i Q_{\lambda} \alpha} \lambda, \ldots\right)
$$

where $Q_{\lambda}$ is the charge of $\lambda$. This is true in particular for the beta-function of $q$. However in terms of the $\tau$ variable

$$
\beta_{2 \pi i \tau}=\frac{d}{d \ln \left(M / M^{\prime}\right)} 2 \pi i \tau^{\prime}=\frac{d}{d q} 2 \pi i \tau^{\prime} \beta_{q}=q^{-1} \beta_{q} .
$$

The additional $q$ factor makes the $\tau$ beta-function invariant. In what follows only the gauge beta-function is considered since the argument for the other superpotential couplings is similar and known, i.e. there are no perturbative [6] or 
non-perturbative [19] corrections to the usual superpotential. The subscript is dropped and it is denoted as $\beta$.

First consider $U(1)_{R}$ invariance. It requires that

$$
\beta=\tilde{f}\left(\frac{q}{m_{*}^{b_{0}}}, \frac{m_{i j}}{m_{*}}, \frac{c_{i}}{m_{*}^{2}}, \lambda_{i j k}\right)
$$

However, the variables of $\tilde{f}$ are not $U(1)$ invariant. They have charges $6 t_{a d j}, 0,3,-3$, respectively. Invariance under $U(1)_{R} \times U(1)$ requires that $\beta$ is a yet another function

$$
\beta=F\left(q \frac{\lambda_{i j k}^{2 t_{a d j}}}{m_{*}^{b_{0}}}, q^{-1} \frac{c_{i}^{2 t_{a d j}}}{m_{*}^{t_{a d j}}+\sum t_{i}}, \frac{m_{i j}}{m_{*}}\right) .
$$

Next take the limit $m_{*} \rightarrow 0$ keeping $q$ and all the arguments of $F$ constant. If $b_{0}>0$, this corresponds to taking all couplings except $\tau$ to zero. Assuming that $\beta$ is continuous it is found that $\beta\left(q, \lambda_{i j k}, m_{i j}, c_{i}\right)=\beta\left(q, \lambda_{i j k}=m_{i j}=c_{i}=0\right)$ and thus it is independent of all the couplings in the superpotential. In fact when the superpotential vanishes it is known [20] that there are no non-perturbative corrections to the beta-function and the gauge coupling only runs at $1-$ loop $^{2}$. This just reflects the fact that no $U(1)_{R} \times U(1)$ holomorphic invariant can be constructed solely in terms of $q$. Note the importance of holomorphy in these arguments. For example, if holomorphy is not required $q \bar{q}$ is invariant under an arbitrary $U(1)$ and $U(1)_{R}$ symmetry. So no higher loops or non-perturbative corrections are present

\footnotetext{
${ }^{2}$ Note that this result can also be written as $\frac{d}{d t} g=-\frac{b_{0}}{16 \pi^{2}} g^{3}$ which is just the standard 1-loop beta-function.
} 
and it is concluded that

$$
\beta=b_{0}
$$

thus extending the perturbative result of Shifman and Vainshtein [17].

An exception to the previous argument occurs when the gauge and global symmetries of the theory allow only Yukawa couplings to be present in the superpotential. For these theories

$$
\beta=f\left(q, \lambda_{i j k}\right)
$$

The beta-function must be $U(1)_{R}$ invariant. This requires

$$
f\left(e^{2 b_{0} \alpha i / 3} q, \lambda_{i j k}\right)=f\left(q, \lambda_{i j k}\right)
$$

Then by holomorphy $\beta$ is independent of $q$. Further, invariance of $\beta$ under the $U(1)$ symmetry requires that $f$ is a function of ratios of $\lambda_{i j k}$ only. Choose one of the non-zero $\lambda_{i j k}, \lambda_{*}$ say, and divide through by $\lambda_{*}$. Then

$$
\beta=f\left(\lambda_{i j k}\right)=F\left(\frac{\lambda_{i j k}}{\lambda_{*}}\right)
$$

Consider the limit $\lambda_{i j k} \rightarrow 0$ while keeping the ratios $\lambda_{i j k} / \lambda_{*}$ constant. In this limit $\beta$ reduces to the one-loop result. So assuming that $\beta$ is continuous, it follows that $\beta\left(\lambda_{i j k}\right)=\beta\left(\lambda_{i j k}=0\right)=b_{0}$, i.e. it is independent of the Yukawa couplings.

To conclude this section, it is noted that the discussion of the proof of the theorem was divided into two cases requiring separate proofs. Here a short argument is presented which extends the proof of the theorem, valid when at least one mass term is allowed, to theories which do not admit any bare mass terms. Consider 
a theory with Lagrangian $\mathcal{L}$ for which the symmetries of the theory forbid the presence of any mass terms. To this theory, add a non-interacting gauge-singlet field with mass $m_{*}$. More concretely, the new theory defined at $M$ is described by the Lagrangian

$$
\mathcal{L}_{\text {new }}=\mathcal{L}+\int d^{2} \theta d^{2} \bar{\theta} \Phi_{0}^{\dagger} \Phi_{0}+\left(\int d^{2} \theta M m_{*} \Phi_{0}^{2}+\text { h.c. }\right)
$$

This new theory satisfies the conditions of the theorem proven when at least one mass term is allowed, so the beta-function of the new theory, $\beta_{n e w}$, is exhausted at one-loop. But on physical grounds it can be concluded that $\beta_{\text {new }}$ is identical to $\beta$, the beta-function of the original theory, since in integrating over momentum modes $M$ to $M^{\prime}$ the contribution from the gauge singlet completely factors out since it is non-interacting. So by this argument the proof of the theorem for theories with mass terms can be extended to theories for which mass terms are forbidden by the symmetries of the model.

The results of this section are also valid for a semi-simple gauge group. The proof of this is sketched in the next section.

\subsection{Extension to a semi-simple gauge group}

Assume that the gauge group is $G=\Pi_{A} G_{A}$ with each $G_{A}$ a simple group. Also assume that the superpotential has the form given in Section 2.2. Then if all the simple gauge groups are asymptotically-free the Wilsonian beta-functions of all the gauge couplings are one-loop exact. 
For each simple gauge group $G_{A}$ define

$$
\tau_{A}=\frac{\theta_{A}}{2 \pi}+\frac{4 \pi i}{g_{A}^{2}}
$$

and introduce $q_{A} \equiv e^{2 \pi i \tau_{A}}$ as in Section 2.2. The $U(1)_{R} \times U(1)$ selection rules of Section 2.2 are extended by assigning all gauge chiral multiplets $W_{\alpha, A}$ charge $(1,0)$. Then $q_{A}$ has charge $\left(2 b_{0}^{A} / 3,2 \sum_{i} t_{A}\left(R_{i}\right)\right)$. It will be convenient to define $\kappa_{A} \equiv\left(q_{A}\right)^{\frac{1}{b_{0}^{A}}}$. Then $\kappa_{A}$ has charge $\left(2 / 3,2 \sum_{i} t_{A}\left(R_{i}\right) / b_{0}^{A}\right)$. Weak coupling is at $\kappa_{A}=0$ since $b_{0}^{A}$ is positive.

The beta-functions for each simple gauge group are defined as in Section 2.2, so that

$$
\beta_{A}=f_{A}\left(q_{B}, \lambda_{i j k}, m_{i j}, c_{i}\right)
$$

is a function of holomorphic invariants and invariant under the $U(1)_{R} \times U(1)$ symmetry.

The proof is done for two cases:

1. Only Yukawa couplings are allowed.

2. At least one $m_{i j} \neq 0$ is allowed.

In the first case invariance of $\beta_{A}$ under $U(1)_{R}$ requires that $\beta_{A}$ is a function of ratios of $\kappa_{B}$ only. That is,

$$
\beta_{A}=F_{A}\left(\kappa_{B} / \kappa_{B_{*}}, \lambda_{i j k}\right)
$$

Here an arbitrarily chosen $\kappa_{B_{*}}$ is selected and divided into the other $\kappa_{B} \mathrm{~s}$, so that each $\kappa_{B}$ other than $\kappa_{B_{*}}$ appears in the argument of $F$ only once. Now consider 
the weak coupling limit $\kappa_{B} \rightarrow 0$ for all the gauge couplings. The argument of the beta-functions is

$$
\kappa_{B} / \kappa_{B_{*}}=\exp 2 \pi i\left(\tau_{B} / b_{0}^{B}-\tau_{B_{*}} / b_{0}^{B_{*}}\right)
$$

Since by assumption the one-loop beta-functions all have the same sign it is possible to take this limit while keeping the ratios $\kappa_{B} / \kappa_{B *}$ fixed. In this limit the beta-function is a function of the Yukawa couplings only. So assuming that the beta-functions are continuous in this limit, it is found that $\beta_{A}\left(\kappa_{B}, \lambda_{i j k}\right)=\beta_{A}\left(\kappa_{B}=\right.$ $\left.0, \lambda_{i j k}\right)=F_{A}\left(\lambda_{i j k}\right)$. But $U(1)$ symmetry may be used to conclude that $\beta_{A}$ is a function of $\lambda_{i j k} / \lambda_{*}$. The argument of Section 2.2 may now be repeated to conclude that $\beta_{A}\left(q_{B}, \lambda_{i j k}\right)=$ constant.

For the second case a straightforward generalization of the argument of Section 2.2 may be repeated with the conclusion that

$$
\beta_{A}=F_{A}\left(\frac{\kappa_{B}}{\kappa_{B_{*}}}\right)
$$

Then the argument used in the first case of this Section is used to conclude that $F_{A}$ is independent of all of the $q_{B}$ and superpotential couplings.

\section{Note}

The statement of this theorem for the case of a simple gauge group was also made in the lecture notes [24]. In that proof the author considers a superpotential containing no composite operators, i.e. only operators linear in the fundamental fields. Of course such superpotential is not gauge invariant. It is however, only 
used in an intermediate step to simplify the study of the charge assignment for the couplings in the physical gauge invariant superpotential. The $U(1)$ charge of the coupling of a composite operator equals the sum of the charges of the couplings of the fundamental fields entering the composite. In [24] however, it is also assumed that the $U(1)_{R}$ charge of the couplings of composite gauge invariant operators in the superpotential equals the sum of the charges of the couplings of fundamental fields forming the composite. While this is true for usual $U(1)$ symmetries since the superpotential has charge zero and the sum of charges of the couplings must equal minus the sum of charges of the fields entering the composite, for $U(1)_{R}$ symmetries the superpotential has charge two and the arithmetic is more complicated. Because of this, the proof in [24] only works for a superpotential linear in matter fields, i.e. when only gauge singlet chiral superfields are present. We also generalized the theorem to a semi-simple gauge group. 


\section{Chapter 3}

\section{$R$-Parity Violation}

In this chapter it is argued that supersymmetric $R$-parity breaking $\left(R_{p}\right)$ interactions always result in Flavor Changing Neutral Current (FCNC) processes. Within a single coupling scheme, these processes can be avoided in either the charge $+2 / 3$ or the charge $-1 / 3$ quark sector, but not both. These processes are used to place constraints on $R_{p}$ couplings. The constraints on the first and the second generations are better than those existing in the literature. The $\not R_{p}$ interactions may result in new top quark decays. Some of these violate electron-muon universality or produce a surplus of $b$ quark events in $t \bar{t}$ decays. Results from the CDF experiment are used to bound these $\not R_{p}$ couplings.

\subsection{Introduction}

The Minimal Supersymmetric Standard Model (MSSM) with the gauge group $G=S U(3)_{c} \times S U(2)_{L} \times U(1)_{Y}$ contains the Standard Model particles and their superpartners, and an additional Higgs doublet. In order to produce the observed 
spectrum of particle masses, the superpotential is given by

$$
\lambda_{i j}^{L} L_{i} E_{j}^{c} \bar{H}+\lambda_{j k}^{D} \bar{H} Q_{j} D_{k}^{c}+\lambda_{i j}^{U} U_{i}^{c} Q_{j} H+\mu H \bar{H}
$$

where $L=\left(\begin{array}{c}N \\ E\end{array}\right)$ and $Q=\left(\begin{array}{c}U \\ D\end{array}\right)$ denote the chiral superfields containing the lepton and quark $S U(2)_{L}$ doublets and $E^{c}, U^{c}$ and $D^{c}$ are the $S U(2)_{L}$ singlets, all in the weak basis. $H$ and $\bar{H}$ are the Higgs doublets with hypercharges $1 / 2$ and $-1 / 2$ respectively. The $S U(2)_{L}$ and $S U(3)_{c}$ indices are suppressed, and $i, j$ and $k$ are generation indices. However, requiring the Lagrangian to be gauge invariant does not uniquely determine the form of the superpotential. In addition, the following renormalizable terms

$$
\lambda_{i j k} L_{i} L_{j} E_{k}^{c}+\bar{\lambda}_{i j k} L_{i} Q_{j} D_{k}^{c}+\lambda_{i j k}^{\prime \prime} U_{i}^{c} D_{j}^{c} D_{k}^{c}
$$

are allowed ${ }^{1}$. Unlike the interactions of the MSSM, these terms violate lepton number and baryon number. They may be forbidden by imposing a discrete symmetry, $R$-parity, which is $(-1)^{3 B+L+2 S}$ on a component field with baryon number $B$, lepton number $L$ and spin $S$. Whether this symmetry is realized in nature must be determined by experiment. If both lepton and baryon number violating interactions are present, then limits on the proton lifetime place stringent constraints on the products of most of these couplings. So, it is usually assumed that if $R$-parity is violated, then either lepton or baryon number violating interactions, but not both, are present. It is interesting that despite the large limits on the proton lifetime,

\footnotetext{
${ }^{1} \mathrm{~A}$ term $\mu_{i} L_{i} H$ is also allowed. This may be rotated away through a redefinition of the $L$ and $H$ fields [25].
} 
some products of the $R$-parity violating couplings remain bounded only by the requirement that the theory remain perturbative [26]. If either $L_{i} Q_{j} D_{k}^{c}$ or $U_{i}^{c} D_{j}^{c} D_{k}^{c}$ terms are present, flavor changing neutral current (FCNC) processes are induced. It has been assumed that if only one $R$-parity violating $\left(R_{p}\right)$ coupling with a particular flavor structure is non-zero, then these flavor changing processes are avoided. In this single coupling scheme [27] then, efforts at constraining $R$-parity violation have concentrated on flavor conserving processes $[28,29,30,31,32,33]$. It is surprising that, even though individual lepton or baryon number is violated in this scheme, the constraints are rather weak.

In Section 3.2, it is demonstrated that the single coupling scheme cannot be realized in the quark mass basis. Despite the general values the couplings may have in the weak basis, after electroweak symmetry breaking there is at least one large $R_{p}$ coupling and many other $R_{p}$ couplings with different flavor structure. Therefore, in the mass basis the $R$-parity breaking couplings cannot be diagonal in generation space. Thus, flavor changing neutral current processes are always present in either the charge $2 / 3$ or the charge $-1 / 3$ quark sectors. These processes are used to place constraints on $R$-parity breaking. Constrainıs on the first and the second generations that are much stronger than existing limits are obtained.

The recent discovery of the top quark $[34,35]$ with the large mass of $176 \mathrm{GeV}$ opens the possibility for the tree level decays $t \rightarrow \tilde{l}_{i}^{+}+d_{k}$ and $t \rightarrow \overline{\tilde{d}}_{j}+\bar{d}_{k}$ if $R$-parity is broken. If the $R_{p}$ couplings are large enough, then these decay channels may be competitive with the Standard Model decay $t \rightarrow b+W$. As no inconsistencies 
between the measured branching fractions and production cross-section of the top quark and those predicted by the Standard Model (SM) have been reported, limits on the branching fractions for the $\not R_{p}$ decay channels may be obtained. Since the existing lower bound on the mass of the lightest slepton is $\sim 45 \mathrm{GeV}$ [36], while the strong interactions of the squarks make it likely that the squarks are heavier than the sleptons, the decay $t \rightarrow \tilde{l}_{i}^{+}+d_{k}$ is more probable. In this analysis, it is therefore assumed that only the slepton decay channel is present. In Section 3.3 the $\not R_{p}$ top decay channels are analyzed to place constraints on the $t \rightarrow \tilde{l}_{i}^{+}+d_{k}$ coupling. For this reason, in this chapter only the $\not{H}$ terms $L_{i} Q_{j} D_{k}^{c}$ are assumed to be present. The conclusions of Section 3.2, however, are valid even if the $L_{i} L_{j} E_{k}^{c}$ terms are also present. Constraints on products of couplings when both $\not$ interactions are present may be found in reference [37]. Section 3.4 summarizes the results and compares them with limits existing in the literature.

\subsection{Flavor Changing Neutral Current Processes}

Flavor changing neutral current processes are more clearly seen by examining the structure of the interactions in the quark mass basis. In this basis, the $\bar{\lambda}_{i j k}$ interactions are

$$
\lambda_{i j k}^{\prime}\left(N_{i}^{m}\left(V_{K M}\right)_{j l} D_{l}^{m}-E_{i}^{m} U_{j}^{m}\right) D_{k}^{c m}
$$

where

$$
\lambda_{i j k}^{\prime}=\bar{\lambda}_{i m n} U_{L j m} D_{R n k}^{*}
$$


The superfields in Eqn.(3.3) have their fermionic components in the mass basis so that the Cabibbo-Kobayashi-Maskawa (CKM) matrix [3] $V_{K M}$ appears explicitly. The rotation matrices $U_{L}$ and $D_{R}$ appearing in the previous equation are defined by

$$
\begin{array}{r}
u_{L i}=U_{L i j} u_{L j}^{m} \\
d_{R i}=D_{R i j} d_{R j}^{m}
\end{array}
$$

where $q_{i}\left(q_{i}^{m}\right)$ are quark fields in the weak (mass) basis. Henceforth, all the fields will be in the mass basis and the superscript $m$ is dropped.

Unitarity of the rotation matrices implies that the couplings $\lambda_{i j k}^{\prime}$ and $\bar{\lambda}_{i j k}$ satisfy

$$
\sum_{j k}\left|\lambda_{i j k}^{\prime}\right|^{2}=\sum_{m n}\left|\bar{\lambda}_{i m n}\right|^{2}
$$

So any constraint on the $\not \ell_{p}$ couplings in the quark mass basis also places a bound on the $R_{p}$ couplings in the weak basis.

In terms of component fields, the interactions are

$$
\lambda_{i j k}^{\prime}\left[\left(V_{K M}\right)_{j l}\left(\tilde{\nu}_{L}^{i} \bar{d}_{R}^{k} d_{L}^{l}+\tilde{d}_{L}^{l} \bar{d}_{R}^{k} \nu_{L}^{i}+\left(\tilde{d}_{R}^{k}\right)^{*} \overline{\left(\nu_{L}^{i}\right)^{c}} d_{L}^{l}\right)-\tilde{e}_{L}^{i} \bar{d}_{R}^{k} u_{L}^{j}-\tilde{u}_{L}^{j} \bar{d}_{R}^{k} e_{L}^{i}-\left(\tilde{d}_{R}^{k}\right)^{*} \overline{\left(e_{L}^{i}\right)^{c}} u_{L}^{j}\right]
$$

where $e$ denotes the electron and $\dot{\tilde{e}}$ its scalar partner and similarly for the other particles.

The contributions of the $R$-parity violating interactions to low energy processes involving no sparticles in the final state arise from using the $R_{p}$ interactions an even number of times. If two $\lambda^{\prime} \mathrm{s}$ or $\lambda^{\prime \prime} \mathrm{s}$ with different flavor structure are non-zero, 
flavor changing low energy processes can occur. These processes are considered in references [25] and [38], respectively. Therefore, it is usually assumed that either only one $\lambda^{\prime}$ with a particular flavor structure is non-zero, or that the $R$-parity breaking couplings are diagonal in generation space. However, Eqn.(3.8) indicates that this does not imply that there is only one set of interactions with a particular flavor structure, or even that they are diagonal in flavor space. In fact, in this case of one $\lambda_{i j k}^{\prime} \neq 0$, the CKM matrix generates couplings involving each of the three down-type quarks. Thus, flavor violation occurs in the down quark sector, though suppressed by the small values of the off-diagonal CKM elements. Below, these processes are used to obtăın constraints on $R$-parity breaking, assuming only one $\lambda_{i j k}^{\prime} \neq 0$.

It would be more natural to assume that there is only one large $\not \ell_{p}$ coupling in the weak basis, i.e., only one $\bar{\lambda}_{i j k} \neq 0$. As has been indicated, this generates many couplings with different flavor structure in the mass basis, e.g., many $\lambda_{i m n}^{\prime}$ s. It is possible that

$$
\lambda_{i m n}^{\prime} \simeq \bar{\lambda}_{i j k} V_{K M j m} \delta_{k n}
$$

This will be the case if, for example, the rotation to the mass basis occurs only for the charge $+2 / 3$ quark sector. Then, in addition to the Feynman diagrams that contribute to the flavor changing neutral current processes when only one $\lambda_{i j k}^{\prime}$ is present, there are new contributions involving the $\lambda_{\text {imn }}^{\prime}(m \neq j, n=k)$ vertices. However, these new contributions interfere constructively with the operators that are present in the effective Lagrangian that is generated when there is only one 
non-zero $\lambda_{i j k}^{\prime}$. So if these more natural assumptions are made, any constraint found for $\bar{\lambda}_{i j k}$ is slightly better than the constraint that is obtained when only one $\lambda_{i j k}^{\prime}$ is present.

It would seem that the flavor changing neutral current processes may be rotated away by making a different physical assumption concerning which $\not \ell_{p}$ coupling is non-zero. For example, while leaving the quark fields in the mass basis, Eqn.(3.3) gives

$$
\begin{aligned}
W_{\not \lambda_{p}} & =\lambda_{i j k}^{\prime}\left(N_{i}\left(V_{K M}\right)_{j l} D_{l}-E_{i} U_{j}\right) D_{k}^{c} \\
& =\left(\lambda_{i j k}^{\prime} V_{K M j l}\right)\left(N_{i} D_{l}-E_{i}\left(V_{K M l p}^{-1}\right) U_{p}\right) D_{k}^{c} \\
& =\tilde{\lambda}_{i j k}\left(N_{i} D_{j}-E_{i}\left(V_{K M j p}^{-1}\right) U_{p}\right) D_{k}^{c}
\end{aligned}
$$

where

$$
\tilde{\lambda}_{i j k} \equiv \lambda_{i m k}^{\prime}\left(V_{K M}\right)_{m j}
$$

With the assumption that the $\lambda_{i j k}^{\prime}$ coefficients have values such that only one $\tilde{\lambda}_{i j k}$ is non-zero, there is only one interaction of the form $N_{L} D_{L} D^{c}$. There is then no longer any flavor violation in the down-quark sector. In particular, there are no $R_{p}$ contributions to the processes discussed below. But now there are couplings involving each of the three up type quarks. So these interactions contribute to FCNC in the up sector; for example, $D^{0}-\bar{D}^{0}$ mixing. We use $D^{0}-\bar{D}^{0}$ mixing to place constraints on $R$-parity violation assuming only one $\tilde{\lambda}_{i j k} \neq 0$. Thus, there is no basis in which FCNC can be avoided in both sectors.

The conclusion that FCNC constraints always exist in either the charged $-1 / 3$ 


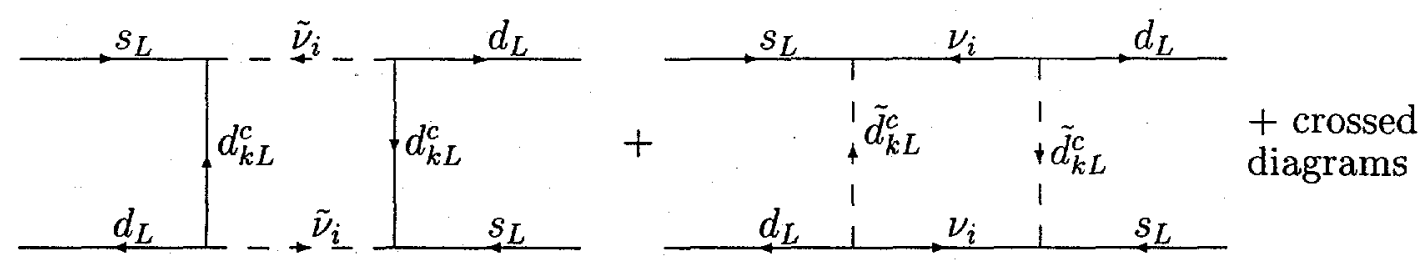

Figure 3.1: $\not R_{p}$ contributions to $K^{0}-\bar{K}^{0}$ mixing with one $\lambda_{i j k}^{\prime} \neq 0$. Arrows indicate flow of propagating left handed fields.

or charged $2 / 3$ quark sectors follows solely from requiring consistency with electroweak symmetry breaking, and is not specific to $R$-parity violation. For example, a similar conclusion about leptoquark interactions, which are similar to $R_{p}$ interactions, is reached in reference [39]. ${ }^{2}$

\subsection{1 $K^{0}-\bar{K}^{0}$ Mixing}

With one $\lambda_{i j k}^{\prime} \neq 0$, the interactions of Eqn.(3.8) involve down and strange quarks. So, there are contributions to $K^{0}-\bar{K}^{0}$ mixing through the box diagrams shown in Figure 3.1. A constraint on the $\not \not_{p}$ couplings is obtained by constraining the sum of the $R_{p}$ and Standard Model contributions to the $K_{L}-K_{S}$ mass difference to be less than the measured value.

Evaluating these diagrams at zero external momentum and neglecting the down quark masses, the following effective Hamiltonian is generated

$$
\mathcal{H}_{\not_{p}}^{\Delta S=2}=\frac{1}{128 \pi^{2}}\left|\lambda_{i j k}^{\prime}\right|^{4}\left(\frac{1}{m_{\bar{\nu}_{i}}^{2}}+\frac{1}{m_{\tilde{d}_{R k}}^{2}}\right)\left(\left(V_{K M}\right)_{j 2}\left(V_{K M}\right)_{j 1}^{*}\right)^{2}\left(\bar{d}_{L} \gamma^{\mu} s_{L}\right)^{2}
$$

where $m_{\tilde{\nu}_{i}}$ is the sneutrino mass and $m_{\tilde{d}_{R k}}$ is the right-handed down squark mass.

\footnotetext{
${ }^{2}$ The author thanks Y. Grossman for bringing this work to his attention.
} 
As this operator is suppressed by the CKM angles, it is largest when $\lambda_{i j k}^{\prime}$ is nonzero for $j=1$ or $j=2$.

The Standard Model effective Hamiltonian is [40]

$$
\mathcal{H}_{S M}^{\Delta S=2}=\frac{G_{F}^{2}}{4 \pi^{2}} m_{c}^{2}\left(\left(V_{K M}\right)_{12}\left(V_{K M}\right)_{11}^{*}\right)^{2}\left(\bar{d}_{L} \gamma^{\mu} s_{L}\right)^{2}
$$

where the CKM suppressed top quark contribution, the up quark mass, QCD radiative corrections, and long distance effects have been ignored.

The $\Delta S=2$ effective Hamiltonian is then

$$
\begin{aligned}
\mathcal{H}^{\Delta S=2} & =\mathcal{H}_{S M}^{\Delta S=2}+\mathcal{H}_{\bar{R}_{p}}^{\Delta S=2} \\
& \equiv G\left(\tilde{\lambda}_{i j k}, m_{\tilde{l}_{i}}, m_{\tilde{d}_{R k}}, V_{K M}\right)\left(\bar{d}_{L} \gamma^{\mu} s_{L}\right)^{2}
\end{aligned}
$$

In the vacuum saturation approximation, this effective Hamiltonian contributes an amount

$$
(\Delta m)_{t h} \equiv m_{K_{L}}-m_{K_{S}}=\frac{2}{3} f_{K}^{2} m_{K} B_{K} \operatorname{Re} G\left(\tilde{\lambda}_{i j k}, m_{\tilde{l}_{i}}, m_{\tilde{d}_{R k}}\right)
$$

to the $K_{L}-K_{S}$ mass difference. With $f_{K}=160 \mathrm{MeV}$ [41], $B_{K} \sim 0.6$ [42], $m_{K}=497 \mathrm{MeV}[36]$, and $\left|(\Delta m)_{e x p}\right|=3.510 \times 10^{-12} \mathrm{MeV}[36]$, and $m_{c} \geq 1.0 \mathrm{GeV}$, the constraint is

$$
\left|\lambda_{i j k}^{\prime}\right| \leq 0.11\left(\frac{1}{z_{i}^{2}}+\frac{1}{w_{k}^{2}}\right)^{-\frac{1}{4}}
$$

where $z_{i}=m_{\tilde{\nu}_{i}} /(100 \mathrm{GeV})$ and $w_{k}=m_{\tilde{d}_{R k}} /(100 \mathrm{GeV})$. This constraint applies for $j=1$ or $j=2$ and for any $i$ or $k$. The constraint for $j=3$ is not interesting as the CKM angles suppress the $\not R_{p}$ operator relative to the Standard Model operator. 


\subsection{2 $\quad B^{0}-\bar{B}^{0}$ Mixing}

The $\not R_{p}$ interactions also contribute to both $B^{0}-\bar{B}^{0}$ mixing and $B_{s}^{0}-\bar{B}_{s}^{0}$ mixing through box diagrams similar to those given in the previous section. As $B_{s}^{0}-\bar{B}_{s}^{0}$ mixing is expected to be nearly maximal, it is not possible at present to place a constraint on any non-Standard Model effects that would add more mixing. However, $B^{0} \bar{B}^{0}$ mixing has been observed [43] with a moderate $x_{d} \equiv \Delta m_{B} / \Gamma_{B}=\sim$ $0.7[36]$.

The effective Hamiltonian generated by these $\not R_{p}$ processes is

$$
\mathcal{H}_{R_{p}}=\frac{1}{128 \pi^{2}}\left|\lambda_{i j k}^{\prime}\right|^{4}\left(\frac{1}{m_{\bar{\nu}_{i}}^{2}}+\frac{1}{m_{\tilde{d}_{R k}}^{2}}\right)\left(\left(V_{K M}\right)_{j 3}\left(V_{K M}\right)_{j 1}^{*}\right)^{2}\left(\bar{d}_{L} \gamma^{\mu} b_{L}\right)^{2}
$$

This is largest when $\lambda_{i 3 k}^{\prime}$ is non-zero.

The dominant contribution to $B^{0}-\bar{B}^{0}$ mixing in the Standard Model is [44]

$$
\mathcal{H}_{S M}^{\Delta S=2}=\frac{G_{F}^{2} m_{t}^{2}}{4 \pi^{2}}\left(\left(V_{K M}\right)_{33}\left(V_{K M}\right)_{31}^{*}\right)^{2} G\left(x_{t}\right)\left(\bar{d}_{L} \gamma^{\mu} b_{L}\right)^{2}
$$

where $x_{t}=m_{t}^{2} / m_{W}^{2}$, and

$$
G(x)=\frac{4-11 x+x^{2}}{4(x-1)^{2}}-\frac{3 x^{2} \ln x}{2(1-x)^{3}}
$$

For a top mass of $176 \mathrm{GeV}, G\left(x_{t}\right)=0.54$.

A constraint for $\lambda_{i 3 k}^{\prime}$ is obtained by demanding that the sum of the Standard Model and $\not R_{p}$ contributions to the $B_{L}-B_{S}$ mass difference not exceed the measured value. With $f_{B}=200 \mathrm{MeV}[41], B_{B} \sim 1.2$ [45], $m_{B}=5279 \mathrm{MeV}[36]$, $\left|(\Delta m)_{e x p}\right|=3.3 \times 10^{-10} \mathrm{MeV}[36]$ and $\left|V_{K M 13}\right| \geq 0.004$ [36], a conservative con- 


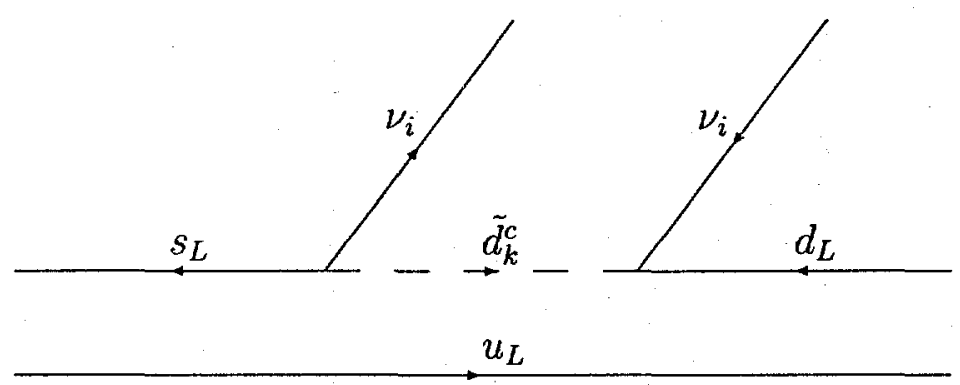

Figure 3.2: $\not R_{p}$ contribution to $K^{+} \rightarrow \pi^{+} \nu \bar{\nu}$ with one $\lambda_{i j k}^{\prime} \neq 0$

straint is

$$
\left|\lambda_{i 3 k}^{\prime}\right| \leq 1.1\left(\frac{1}{z_{i}^{2}}+\frac{1}{w_{k}^{2}}\right)^{-\frac{1}{4}}
$$

with $z_{i}$ and $w_{k}$ as previously defined. In this case the $\not R_{p}$ couplings are only weakly constrained.

In addition to inducing $B^{0}-\bar{B}^{0}$ mixing, these interactions also contribute to the $b \rightarrow s+\gamma$ amplitude. However, with reasonable values for squark and sneutrino masses, the constraint is significantly weaker than that found from the top quark analysis.

\subsection{3 $K^{+} \rightarrow \pi^{+} \nu \bar{\nu}$}

The tree-level Feynman diagram in Figure 3.2 generates an effective Hamiltonian which contributes to the branching ratio for $K^{+} \rightarrow \pi^{+} \nu \bar{\nu}$. Using a Fierz rearrangement, a straightforward evaluation of this diagram gives

$$
\mathcal{H}_{\not_{p}}=\frac{1}{2} \frac{\left|\lambda_{i j k}^{\prime}\right|^{2}}{m_{\tilde{d}_{R k}}^{2}}\left(V_{K M j 1} V_{K M j 2}^{*}\right)\left(\bar{s}_{L} \gamma^{\mu} d_{L}\right)\left(\bar{\nu}_{L i} \gamma_{\mu} \nu_{L i}\right)
$$

There is also a Standard Model contribution to this decay [44]. This is an 
order of magnitude lower than the existing experimental limit. A bound on the $\not R_{p}$ coupling is obtained by assuming that the $\not R_{p}$ effects dominate the decay rate. As the matrix element for this semileptonic decay factors into a leptonic and a hadronic element, the isospin relation

$$
\left\langle\pi^{+}(\mathbf{p})\left|\overline{\mathbf{s}} \gamma_{\mu} \mathrm{d}\right| \mathrm{K}^{+}(\mathbf{k})\right\rangle=\sqrt{2}\left\langle\pi^{0}(\mathbf{p})\left|\overline{\mathbf{s}} \gamma_{\mu} \mathrm{u}\right| \mathrm{K}^{+}(\mathbf{k})\right\rangle
$$

can be used to relate $\Gamma\left[K^{+} \rightarrow \pi^{+} \nu \bar{\nu}\right]$ to $\Gamma\left[K^{+} \rightarrow \pi^{0} \nu e^{+}\right]$. The effective Hamiltonian for the neutral pion decay channel arises from the spectator decay of the strange quark. It is

$$
\mathcal{H}_{e f f}=\frac{4 G_{F}}{\sqrt{2}} V_{K M 12}^{*}\left(\bar{s}_{L} \gamma^{\mu} u_{L}\right)\left(\bar{\nu}_{L i} \gamma_{\mu} e_{L i}\right)
$$

So in the limit where the lepton masses can be neglected,

$$
\frac{\Gamma\left[K^{+} \rightarrow \pi^{+} \nu_{i} \bar{\nu}_{i}\right]}{\Gamma\left[K^{+} \rightarrow \pi^{0} \nu e^{+}\right]}=\left(\frac{\left|\lambda_{i j k}^{\prime}\right|^{2}}{4 G_{F} m_{\tilde{d}_{R k}}^{2}}\right)^{2}\left(\frac{\left|V_{K M j 1} V_{K M j 2}^{*}\right|}{\left|V_{K M 12}^{*}\right|}\right)^{2} .
$$

This ratio is valid for $i=1,2$ or 3 , since in the massless neutrino and electron approximation, the integrals over phase space in the numerator and denominator cancel. So using $B R\left[K^{+} \rightarrow \pi^{+} \nu \bar{\nu}\right] \leq 5.2 \times 10^{-9}[46](90 \% C L)$ and $B R\left[K^{+} \rightarrow\right.$ $\pi^{0} \nu e^{+} j=0.0482[36]$, the constraint is

$$
\left|\lambda_{i j k}^{\prime}\right| \leq 0.012\left(\frac{m_{\tilde{d}_{R k}}}{100 G e V}\right)(90 \% C L)
$$

for $j=1$ or $j=2$. Using $\left|V_{K M 13}\right| \geq 0.004$ [36] and $\left|V_{K M 23}\right| \geq 0.03$ [36], a conservative upper bound for $\lambda_{i 3 k}^{\prime}$ is

$$
\left|\lambda_{i 3 k}^{\prime}\right| \leq 0.52\left(\frac{m_{\tilde{d}_{R k}}}{100 G e V}\right)(90 \% C L) .
$$




\subsection{4 $D^{0}-\bar{D}^{0}$ Mixing}

If there is only one $\tilde{\lambda}_{i j k}$ in the mass basis, then from Eqn.(3.12) it is clear that flavor changing neutral current processes will occur in the charge $+2 / 3$ quark sector. Rare processes such as $D^{0}-\bar{D}^{0}$ mixing, $D^{0} \rightarrow \mu^{+} \mu^{-}$and $D^{+} \rightarrow \pi^{+} l^{+} l^{-}$, for example, may be used to place tight constraints on $\tilde{\lambda}_{i j k}$. For illustrative purposes, in this section $D^{0}-\bar{D}^{0}$ mixing is considered.

The interactions in Eqn.(3.12) generate box diagrams identical to those discussed in the previous sections if both the internal sneutrino (neutrino) propagators are replaced with slepton (lepton) propagators and the external quarks lines are suitably corrected. Using the same approximations that were made earlier, the $\not R_{p}$ effects generate the following effective Hamiltonian

$$
\mathcal{H}_{\not_{p}}=\frac{1}{128 \pi^{2}}\left|\tilde{\lambda}_{i j k}\right|^{4}\left(\frac{1}{m_{\tilde{l}_{i}}^{2}}+\frac{1}{m_{\tilde{d}_{R k}}^{2}}\right)\left(\left(V_{K M}\right)_{2 j}\left(V_{K M}\right)_{1 j}^{*}\right)^{2}\left(\bar{c}_{L} \gamma^{\mu} u_{L}\right)^{2}
$$

With $f_{D}=200 \mathrm{MeV}[41], m_{D}=1864 \mathrm{MeV}$ [36], and $\left|(\Delta m)_{\exp }\right| \leq 1.32 \times$ $10^{-10} \mathrm{MeV}[36](90 \% C L)$, the constraint on $\tilde{\lambda}_{i j k}$ for $j=1$ or $j=2$ is

$$
\left|\tilde{\lambda}_{i j k}\right| \leq 0.16\left(\left(\frac{100 \mathrm{GeV}}{m_{\tilde{l}_{i}}}\right)^{2}+\left(\frac{100 \mathrm{GeV}}{m_{\tilde{d}_{R k}}}\right)^{2}\right)^{-\frac{1}{4}}(90 \% C L) .
$$

\subsection{Top Quark Decay}

In the Standard Model, the dominant decay mode for the top quark is

$$
t \rightarrow b+W
$$


with a real $W$ gauge boson produced. This has a partial decay width

$$
\Gamma[t \rightarrow W+b]=\frac{G_{F} m_{t}^{3}}{8 \pi \sqrt{2}}\left|V_{t b}\right|^{2}\left(1-x_{W}^{2}\right)\left(1-2 x_{W}^{4}+x_{W}^{2}\right)
$$

where $x_{W}=m_{W} / m_{t}$. The $b$ quark mass has been neglected.

The $R$-parity violating interactions (see Eqn.(3.8) with $j=3$ ) $\lambda_{i 3 k}^{\prime} \tilde{e}_{L}^{i} \bar{d}_{R}^{k} t_{L}$ contribute to the decay $t_{L} \rightarrow \tilde{l}_{i}^{+}+d_{R k}$ at tree level [47], if kinematically allowed. This is possible only if there exist sleptons lighter than the top quark. The partial width for this process is

$$
\Gamma\left[t \rightarrow \tilde{l}_{i}^{+}+d_{k}\right]=\frac{\left|\lambda_{i 3 k}^{\prime}\right|^{2} m_{t}\left(1-y_{i}^{2}\right)^{2}}{32 \pi}
$$

with $y_{i} \equiv m_{\tilde{l}_{i}} / m_{t}[47]$. The mass of the down type quark has been neglected. If this is the only non-zero $R$-parity coupling, the two top quark decay channels are $t \rightarrow b+W$ and $t \rightarrow d_{R k}+\tilde{l}_{i}^{+}$, with branching fractions $1-x$ and $x$, respectively.

The Lightest Supersymmetric Particle (LSP), denoted by $\tilde{\chi}^{0}$, is assumed to be neutral and that the real slepton decays with $100 \%$ branching fraction to the $\tilde{\chi}^{0}$ and a lepton. The presence of a non-zero $R$-parity breaking coupling implies that the $\tilde{\chi}^{0}$ is no longer stable [5]. The two dominant decays are [47] $\tilde{\chi}^{0} \rightarrow \nu_{i}+b+\bar{d}_{k}$ and $\tilde{\chi}^{0} \rightarrow \bar{\nu}_{i}+\vec{b}+d_{k}$. The LSP decays inside the detector if [30]

$$
\left|\lambda_{i 3 k}^{\prime}\right| \geq 6 \times 10^{-5} \sqrt{\gamma}\left(\left(\frac{100 \mathrm{GeV}}{m_{\tilde{d}_{R k}}}\right)^{2}+\left(\frac{100 \mathrm{GeV}}{m_{\tilde{b}_{R}}}\right)^{2}\right)\left(\frac{100 \mathrm{GeV}}{m_{\tilde{\chi}^{0}}}\right)^{5 / 2}
$$

where $\gamma$ is the Lorentz boost factor of $\tilde{\chi}^{0}$. For this decay chain to be kinematically allowed, $m_{\tilde{\chi}^{0}} \geq m_{b}$ for $k=1$ or $k=2$, and $m_{\tilde{\chi}^{0}} \geq 2 m_{b}$ for $k=3$ are required. Using the previous equation, the maximum lower bound on $\lambda_{i 3 k}^{\prime}$ such that the LSP 
decays inside the detector is $0.0003 \times \sqrt{\gamma}$ for $k=3$, and $0.002 \times \sqrt{\gamma}$ for $k=1$ or $k=2$; all for $300 \mathrm{GeV}$ squark masses. In the following $\lambda_{i 3 k}^{\prime}$ is assumed to be larger than this value so that the LSP decays within the detector.

If a top quark decays through this $R$-parity violating process, the final state will contain one lepton, at least one $b$ quark and missing transverse energy. The two novel features of this decay channel are that it spoils lepton universality and, when $k=3$, produces a surplus of $b$ quark events. Both of these signatures can be used to test the strength of $R$-parity violation.

The CDF collaboration reconstructs $t \bar{t}$ quark events from observing: (1) dilepton (electron or muon) events coming from the leptonic decays of both the $W$ 's; or (2) one lepton event arising from leptonic decay of one $W$ and jets from the hadronic decay of the remaining $W$ boson. CDF also requires a $b$-tag in the lepton+jets channel. If the lightest slepton has a mass between 50 and $100 \mathrm{GeV}$, then the kinematics of the decay $\tilde{l}_{i} \rightarrow \tilde{\chi}^{0}+l_{i}$ will be similar to that of the leptonic decay of the $W$ boson. A slepton of mass less than $45 \mathrm{GeV}$ is ruled out by the LEP limit on the $Z$ decay width [36]. If the slepton mass is close to the top mass, then the $b$ quark produced in the top decay via this channel will have less energy than the $b$ quark from the top decay via the SM channel. Also, the lepton from the slepton decay will have more energy than the lepton from the $W$ decay. These will affect the lepton and the $b$. quark detection efficiencies. Although these decay channels will be present for any slepton lighter than the top quark, for the purpose of obtaining a constraint, it is assumed that there is a slepton with a mass in 
the range given above. The presence of the $R$-parity violating coupling will then contribute signals to all of these channels.

It is assumed that the $i=1$ coupling is non-zero. However, all that is required is that the slepton in the generation with the non-zero coupling have a mass in the range quoted above, i.e., if $\lambda_{13 k}^{\prime} \neq 0$ then we require $50 \mathrm{GeV}<m_{\tilde{e}}<100 \mathrm{GeV}$, and if $\lambda_{23 k}^{\prime} \neq 0$ then $50 \mathrm{GeV}<m_{\tilde{\mu}}<100 \mathrm{GeV}$ is required. Assuming also that the CDF data is consistent with lepton universality, the constraints we obtain for $\lambda_{13 k}^{\prime}$ and $\lambda_{23 k}^{\prime}$ are identical.

In the $k=1,2$ cases, two $b$ quarks are always produced in a $t \bar{t}$ event. In the $k=3$ case, the LSP decays into $\bar{b} b \nu_{i}$ or $\bar{b} b \bar{\nu}_{i}$. Thus, four or six $b$ quarks may be produced if one or both of the top quarks decay through the $R$-parity breaking channel; this possibility must be treated separately.

\subsection{1 $\lambda_{i 3 k}^{\prime}, k \neq 3$}

The branching fraction for the di-electron event is

$$
B R[t \bar{t} \rightarrow e e+X]=x^{2}+L^{2}(1-x)^{2}+2 L x(1-x)
$$

with $L=$ leptonic branching fraction of $W$, approximately $1 / 9$. The first term arises from both top quarks decaying via the $R$-parity violating interaction; the second is the Standard Model contribution; and the third is the contribution from one top quark decaying through the $R$-parity breaking channel and the other top quark decaying through the Standard Model channel. The other branching 
fractions are

$$
\begin{aligned}
& B R[t \bar{t} \rightarrow \mu \mu+X]=L^{2}(1-x)^{2} \\
& B R[t \bar{t} \rightarrow \mu e+X]=2(1-x)^{2} L^{2}+2 x(1-x) L \\
& B R[t \bar{t} \rightarrow \mu+\text { jets }]=2(1-x)^{2} L(1-3 L) \\
& B R[t \bar{t} \rightarrow e+\text { jets }]=2(1-x)^{2} L(1-3 L)+2 x(1-x)(1-3 L)
\end{aligned}
$$

The factor of $1-3 L$ is the hadronic branching fraction of the $W$ boson. Also, it is that assumed that the branching fraction for $\tilde{l} \rightarrow l+\tilde{\chi}^{0}$ is close to one. Here the leptonic events produced from the Standard Model decay of the $W$ boson into $\tau \nu_{\tau}$ are ignored.

Two independent constraints on the $\not h_{p}$ interactions may be obtained from the top quark data. CDF has observed the $t \bar{t}$ cross section to be $\sigma(t \bar{t})_{e x p}=6.8_{-2.4}^{+3.6}$ $\mathrm{pb}[35]$. The QCD calculation [48] gives the value $\sigma(t \bar{t})_{t h}=5.52_{-0.45}^{+0.07} \mathrm{pb}$ for $m_{t}=176 \mathrm{GeV}$

The first method is to compare the ratio of theoretically predicted values for the numbers of events found in two channels with the experimentally observed ratio. For example, $\sigma(t \bar{t})_{t h} \times B R[t \bar{t} \rightarrow \mu+$ jets $] \times \int L d t \times($ detection efficiencies $)$ is the number of $\mu+$ jets events that should have been observed where $\int L d t$ is the integrated luminosity. This theoretical prediction contains uncertainties in both the value for the $t \bar{t}$ production cross section and in the lepton and the $b$ quark detection efficiencies. In comparing the ratio

$$
\left(\sigma(t \bar{t})_{t h} \times B R[t \bar{t} \rightarrow e+\mathrm{jets}]\right) /\left(\sigma(t \bar{t})_{t h} \times B R[t \bar{t} \rightarrow \mu+\mathrm{jets}]\right)
$$


the uncertainties in the $t \bar{t}$ cross section cancel. The $b$-detection efficiencies also cancel. If the electron and the muon detection efficiencies in the lepton + jets channel are equal, these uncertainties will also cancel. The only remaining errors are statistical. The CDF collaboration reported observing $37 b$-tagged events in the lepton $+\geq 3$ jets channel. In this set there were $50 b$-tags, with a background of $22 b$-tags. A conservative estimate for the background in the 37 events is 22 . This leaves $15 t \bar{t}$ events in the lepton +jets channel. Since no inconsistencies with electron-muon universality have been reported, a central value of $7 \mu$ jets and $7 e$ +jets events will be assumed. This leads to

$$
\frac{B R[t \bar{t} \rightarrow e+\text { jets }]_{t h}}{B R\left[t \bar{t} \rightarrow \mu+\text { jets }_{t h}\right.}=\frac{\#(e+\text { jets events })}{\#(\mu+\text { jets events })}=1_{-b}^{+a}
$$

Inserting the theoretical predictions for the branching ratios leads to the constraint $x<L a /(1+L a)$, where $a$ is the uncertainty in the previous ratio. In this case, $a=b=1 / \sqrt{7}$. This gives $x<0.077$ at $95 \% C L$ which leads to

$$
\left|\lambda_{13 k}^{\prime}\right| \leq 0.41(95 \% C L)
$$

for $k=1$ or $k=2$ and a slepton of mass $100 \mathrm{GeV}$.

A similar analysis may be performed for the dilepton channels. In principle these channels should lead to a good constraint since a non-zero $\lambda_{13 k}^{\prime}$ coupling will lead to an excess of electrons observed in the di-electron channel over the number of muons observed in the di-muon channel. However at present only a small number of dilepton events have been observed and an interesting constraint cannot be obtained. 
In the other method the number of events produced in a given channel is compared with the theoretical expectation. The number of produced events is $\sigma[t t]_{t h} \times B R[t \rightarrow l+\text { jets }]_{t h} \times \int L d t$. Here $\sigma[t t]_{t h}$ is the production cross section calculated in perturbative QCD for the assumed top quark mass of $176 \mathrm{GeV}$. The fact that the number of experimentally observed events in any given channel is consistent with, within experimental errors, the number expected in the Standard Model will also be used. The actual number of events detected depends upon the detection efficiency. We will use the number of observed events in any channel to determine the statistical accuracy with which the rate in that channel is measured, and then constrain the strength of the $\not R_{p}$ terms by requiring that the rate is not changed by more than the error.

This leads to the constraint

$$
\frac{B R[t \bar{t} \rightarrow l+\text { jets, } x]_{t h}}{B R[t \bar{t} \rightarrow l+\text { jets, } x=0]_{t h}}=\frac{\sigma[t]_{\exp }}{\sigma[t \bar{t}]_{t h}}
$$

within theoretical and experimental errors. Using the theoretical and experimental values for the production cross sections $[35,48]$ leads to

$$
\epsilon^{2} \leq \frac{B R[t \bar{t} \rightarrow l+\text { jets, } x]_{t h}}{B R[t \bar{t} \rightarrow l+\text { jets, } x=0]_{t h}} \leq 1+d
$$

with $\epsilon=0.89$ and $d=1.05$. The constraint on $x$ is then

$$
x \leq \min \left(1-\epsilon, \frac{1-2 L-\sqrt{(1-2 L)^{2}-4 L d(1-L)}}{2(1-L)}\right)
$$

The first entry is the constraint from the $\mu+$ jets channel and the second entry is from the $e+$ jets channel. For these values of $\epsilon$ and $d$, the constraint is $x \leq 0.11$. 
For a $100 \mathrm{GeV}$ slepton this translates into the constraint

$$
\left|\lambda_{13 k}^{\prime}\right| \leq 0.48
$$

for $k=1$ or $k=2$.

\subsection{2 $\lambda_{i 33}^{\prime}$}

For this coupling the analysis of the previous section must be modified in the lepton + jets channel since the $b$-detection efficiencies no longer cancel. This is because in the $R$-parity breaking decay channel three $b$ quarks are produced. To correct for this, introduce the function $P(k, n)$ that gives the probability that, given that $n b$ quarks are produced, $k$ of them are detected. Then the number of observed single $b$ quark events expected in the $e+$ jets channel is

$$
\begin{aligned}
\#(e+\text { jets events })= & \left(2(1-x)^{2} L(1-3 L) P(1,2)+2 x(1-x)(1-3 L) P(1,4)\right) \\
& \times \mathcal{N}
\end{aligned}
$$

where

$$
\mathcal{N} \equiv \int L d t \times \sigma(t)_{t h}
$$

With $P(1,2) \leq P(1, n)$ for $n \geq 2$, then

$$
\#(e+\text { jets events }) \geq\left(2(1-x)^{2} L(1-3 L)+2 x(1-x)(1-3 L)\right) P(1,2) \times \mathcal{N}
$$

These approximations will give a conservative limit for $\lambda_{133}^{\prime}$. The analysis of the previous section may now be carried out with the following restrictions: 
(i) In comparing the ratio of the numbers of events detected in two channels with the theoretical prediction, the inequality in Eqn.(3.50) indicates that only upper limit in Eqn.(3.42) may used;

(ii) In comparing the number of events detected in a channel with the theoretically predicted value for that channel, only the upper bound in Eqn.(3.45) may be used in the $e+$ jets channel, and either limit may be used in the $\mu+$ jets channel. With these caveats, a conservative limit on the branching fraction for $t \rightarrow b+\tilde{l}_{i}^{+}$is then

$$
x \leq \min \left(L a /(1+L a), 1-\epsilon, \frac{1-2 L-\sqrt{(1-2 L)^{2}-4 L d(1-L)}}{2(1-L)}\right)
$$

For the errors quoted in the previous section, the result is

$$
\left|\lambda_{133}^{\prime}\right| \leq 0.41(95 \% C L) .
$$

As the $R$-parity breaking decay channels produce three $b$ quarks, then for moderate values of $\lambda_{133}^{\prime}$ or $\lambda_{233}^{\prime}$, semileptonic events containing four and six $b$ quarks should be observable at the Tevatron. The non-observance of these events should provide the strongest test for the $R$-parity breaking couplings $\lambda_{133}^{\prime}$ or $\lambda_{233}^{\prime}$. If limits on the branching fractions for the $t \bar{t}$ pair to decay into these excess $b$ quark channels are known, then the $R$-parity branching fraction $x$ is ccnstrained. Namely,

$$
\begin{aligned}
& \text { 1. } B R\left[t \bar{t} \rightarrow X+\geq 3 b^{\prime} s\right] \leq B_{1} \Rightarrow x \leq\left(1-\sqrt{1-B_{1}}\right) \\
& \text { 2. } B R\left[t \bar{t} \rightarrow X+\geq 3 b^{\prime} s+2 e\right] \leq B_{2} \Rightarrow x \leq \frac{\sqrt{L^{2}+B_{2}(1-2 L)}-L}{1-2 L} \\
& \text { 3. } B R\left[t \bar{t} \rightarrow X+\geq 6 b^{\prime} s+2 e\right] \leq B_{3} \Rightarrow x \leq \sqrt{B_{3}} \\
& \text { 4. } B R\left[t \bar{t} \rightarrow X+\geq 3 b^{\prime} s+e\right] \leq B_{4} \Rightarrow x \leq \frac{1}{2}\left(1-\sqrt{1-\frac{2 B_{4}}{1-3 L}}\right)
\end{aligned}
$$


This constrains $\left|\lambda_{133}^{\prime}\right|$. To constrain $\left|\lambda_{233}^{\prime}\right|$, interchange $e$ with $\mu$ in the previous equations.

The constraints on $\left|\lambda_{133}^{\prime}\right|$ and $\left|\lambda_{233}^{\prime}\right|$ found in this section are comparable to those obtained from examining $R_{p}$ contributions either to $Z \rightarrow b \bar{b}$ and $Z \rightarrow$ $l^{+} l^{-}$decays [32] or to forward-backward asymmetry measurements $\left(A_{F B}^{e}\right)$ in $e^{+} e^{-}$ collisions [28]. The point of this exercise has been to illustrate how comparable $\not R_{p}$ constraints may be obtained from analyzing top quark decays even though the experimental and theoretical errors are still large. These processes will provide much better tests of $R$-parity violation once more top quark decays are seen.

\subsection{Summary}

In this chapter it has been argued that $R$-parity breaking interactions always lead to flavor changing neutral current processes. It is possible that there is a single $R_{p}$ coupling in the charge $+2 / 3$ quark sector. But requiring consistency with electroweak symmetry breaking demands that $\not R_{p}$ couplings involving all the charge $-1 / 3$ quarks exist. That is, a single coupling scheme may only be possible in either the charge $2 / 3$ or the charge $-1 / 3$ quark sector, but not both. As a result, flavor changing neutral current processes always exist in one of these sectors. The processes $K^{+} \rightarrow \pi^{+} \nu \bar{\nu}, K^{0}-\bar{K}^{0}$ mixing, $B^{0}-\bar{B}^{0}$ mixing and $D^{0}-\bar{D}^{0}$ mixing have been used to constrain the $R_{p}$ couplings. If there is CKM-like mixing in 
the charged $-1 / 3$ quark sector, then the constraints are quite stringent; see Table 3.1. The tightest constraint is on $\left|\lambda_{i j k}^{\prime}\right|$ for $j=1,2$ and any $i$ and $k$. This comes from the rare decay $K^{+} \rightarrow \pi^{+} \nu \bar{\nu}$. The constraints we obtain for the first two generations are more stringent than those presently existing in the literature.

The $R$-parity breaking interactions lead to the top quark decay $t \rightarrow \tilde{l}_{i}+d_{k}$, if the slepton is lighter than the top quark. Some of the new top quark decays spoil electron-muon universality or result in $t \bar{t}$ events with more than $2 b$ quarks. At present, the CDF collaboration has not reported any inconsistencies with lepton universality or reported any events with more than $2 b$ quarks. These decays also lower the branching fractions for Standard Model top quark decays. Both of these observations are used to constrain some $\not R_{p}$ couplings.

A list of the known model independent constraints on the $\lambda_{i j k}^{\prime}$ couplings is presented in Table 3.2. Although several of these couplings are constrained by different low energy processes, only the smallest known upper limit is listed. With the exception of $\lambda_{133}^{\prime}$, the constraints on the third quark generation couplings are only of order $e / \sin \theta_{w}$. Once more top quark decays are observed the signatures discussed in this chapter will more tightly constrain these couplings. 


\begin{tabular}{||l|l||l|l||l|l||}
\hline$\left|\lambda_{1 j k}^{\prime}\right|$ & & $\left|\lambda_{2 j k}^{\prime}\right|$ & & $\left|\lambda_{3 j k}^{\prime}\right|$ & \\
\hline 111 & $0.012^{a}$ & 211 & $0.012^{a}$ & 311 & $0.012^{a}$ \\
\hline 112 & $0.012^{a}$ & 212 & $0.012^{a}$ & 312 & $0.012^{a}$ \\
\hline 113 & $0.012^{a}$ & 213 & $0.012^{a}$ & 313 & $0.012^{a}$ \\
\hline 121 & $0.012^{a}$ & 221 & $0.012^{a}$ & 321 & $0.012^{a}$ \\
\hline 122 & $0.012^{a}$ & 222 & $0.012^{a}$ & 322 & $0.012^{a}$ \\
\hline 123 & $0.012^{a}$ & 223 & $0.012^{a}$ & 323 & $0.012^{a}$ \\
\hline 131 & $0.19^{b}$ & 231 & $0.19^{b}$ & 331 & $0.19^{b}$ \\
\hline 132 & $0.19^{b}$ & 232 & $0.19^{b}$ & 332 & $0.19^{b}$ \\
\hline 133 & $0.001^{c}$ & 233 & $0.19^{b}$ & 333 & $0.19^{b}$ \\
\hline
\end{tabular}

Table 3.1: Constraints on $\left|\lambda_{i j k}^{\prime}\right|$ from:(a) $K^{+} \rightarrow \pi^{+} \nu \bar{\nu}(90 \% C L)$; (b) $b \rightarrow s \nu \bar{\nu}$ $(90 \% C L)[49]$; (c) $\nu_{e}$ mass $(90 \% C L)$ [29]. These constraints were obtained assuming $C K M$-like mixing in the charged $-1 / 3$ quark sector. All limits are for $100 \mathrm{GeV}$ sparticle masses. 


\begin{tabular}{||l|l||l|l||l|l||}
\hline$\left|\lambda_{1 j k}^{\prime}\right|$ & & $\left|\lambda_{2 j k}^{\prime}\right|$ & & $\left|\lambda_{3 j k}^{\prime}\right|$ & \\
\hline 111 & $0.03^{a}$ & 211 & $0.09^{b}$ & 311 & $0.18^{d}$ \\
\hline 112 & $0.03^{a}$ & 212 & $0.09^{b}$ & 312 & $0.18^{d}$ \\
\hline 113 & $0.03^{a}$ & 213 & $0.09^{b}$ & 313 & $0.18^{d}$ \\
\hline 121 & $0.26^{f}$ & 221 & $0.17^{e}$ & 321 & $0.18^{d}$ \\
\hline 122 & $0.45^{c}$ & 222 & $0.17^{e}$ & 322 & $0.18^{d}$ \\
\hline 123 & $0.26^{c}$ & 223 & $0.17^{e}$ & 323 & $0.18^{d}$ \\
\hline 131 & $0.26^{f}$ & 231 & $0.22^{g}$ & 331 & $0.26^{h}$ \\
\hline 132 & $0.4^{i}$ & 232 & $0.4^{i}$ & 332 & $0.26^{h}$ \\
\hline 133 & $0.001^{j}$ & 233 & $0.4^{i}$ & 333 & $0.26^{h}$ \\
\hline
\end{tabular}

Table 3.2: Constraints on $\left|\lambda_{i j k}^{\prime}\right|$ from:(a) charged current universality (95\%CL) [28]; (b) $\Gamma\left(\pi \rightarrow e \nu_{e}\right) / \Gamma\left(\pi \rightarrow \mu \nu_{\mu}\right)(1 \sigma)$ [28]; (c) $A_{F B}^{e}(1 \sigma)[28] ;$ (d) $B R[\tau \rightarrow$ $\left.\pi \nu_{\tau}\right](95 \% C L)$ [31]; (e) $B R\left[D^{0} \rightarrow K^{-} \mu^{+} \nu_{\mu}\right] / B R\left[D^{0} \rightarrow K^{-} e^{+} \nu_{e}\right](95 \% C L)$ [31]; (f) atomic parity violation and $e D$ asymmetry $(1 \sigma)\left[28^{1}\right.$; (g) $\nu_{\mu}$ deep-inelastic scattering $(95 \% C L)$ [28]; (h) partial $Z^{0}$ decay width $(95 \% C L)$ [32]; (i) top quark decay $(95 \% C L)$; (j) $\nu_{e}$ mass $(90 \% C L)$ [29]. All limits are for $100 \mathrm{GeV}$ sparticle masses. 


\section{Chapter 4}

\section{Signals of Supersymmetric Flavor Violation at}

\section{the LHC}

In a generic supersymmetric extension of the Standard Model, there will be lepton flavor violation at a neutral gaugino vertex due to misalignment between the lepton Yukawa couplings and the slepton soft masses. Sleptons produced at the LHC through the cascade decays of squarks and gluinos can give a sizable number of events with 4 leptons. This channel could give a clean signature of supersymmetric lepton flavor violation under conditions which are identified.

\subsection{Introduction}

In the supersymmetric Standard Model (SM), the quadratically divergent corrections to the Higgs (mass) ${ }^{2}$ cancel due to supersymmetry (SUSY). The remaining corrections are logarithmically divergent, proportional to the SUSY ureaking masses of the sparticles (the superpartners of the SM particles) and result in a negative Higgs (mass) ${ }^{2}$ due to the large top quark Yukawa coupling. Thus, the superpartners of the SM particles must have masses $\lesssim 1 \mathrm{TeV}$ in order for SUSY to solve the gauge hierarchy problem and lead to natural electroweak symmetry 
breaking.

With the sparticle masses at the weak scale, these new particles (especially gluinos and squarks) will be produced in significant amounts at the LHC. After the initial discovery of the sparticles, the focus will be on precision measurements of their masses and mixings just as, for example, the next step after the discovery of the heavy quarks was the measurement of their detailed properties. In this chapter, a relatively clean signal at the LHC for detecting the mixing angle between the scalar partners of the charged leptons (the sleptons) is presented.

A flavor-violating signal is obtained from the production of real sleptons, followed by their oscillation into a different flavored slepton, and subsequent decay to a lepton. Some formulae for these oscillations are given in section 4.1.1. At a $e e$ linear collider, the production of slepton pairs can then give $e \mu$ events with missing energy. This was studied in $[50,51]$. Dilepton flavor and CP violating signals at the LHC and NLC were studied in [52]. At a hadron collider (the LHC), sleptons can be pair-produced by the Drell-Yan process giving the same signal. This was studied in $[53,54]$, and is a promising signal for large flavor mixing angles and when the SUSY background is known to be smell. Real sleptons can also be produced at the LHC in the decays of the next-to-lightest neutralino $\left(\chi_{2}^{0}\right)$, which are mainly produced in the cascade decays of gluinos and squarks. In section 4.3.1, flavor violating dilepton events from $\chi_{2}^{0}$ decays are briefly considered. The production of $\chi_{2}^{0}$ pairs can give rise to events with 4 leptons, with the dramatic flavor violating signal identified by a $(3 e+\mu)$ or $(3 \mu+e)$ lepton 
signature, hard jets, no $b$-jets, and of course missing energy. This is discussed in section 4.3.2. Conditions on the supersymmetric spectrum that are favorable for the suppression of the dominant supersymmetric background, occurring from heavier neutralino/chargino and stop decays, are identified. Ideas for determining the remaining dominant supersymmetric background occurring from $\tau$ decays are also presented. These are all conveniently summarized in the end of the chapter.

In section 4.3.2, a brief estimate of the expected 4-lepton signal at a generic point in SUSY parameter space is given. Next, in section 4.3.2, a particular point in the "minimal supergravity" inspired parameter space is considered [55,. 56]. It is found that at this LHC Point, a $5 \sigma$ discovery ( $2 \sigma$ exclusion) is obtained for a right-handed (RH) first and second generation mixing angle $\sin \theta_{R}>0.13$ $\left(\sin \theta_{R}>0.08\right.$ ) with an integrated luminosity of $100 \mathrm{fb}^{-1}$ at low luminosity. The discovery potential at high luminosity is still optimistic though less quantitative, due to uncertainties in $\tau$-jet detection efficiencies and larger $b$-jet mistagging rates. In any case, the values for the mass splitting (between $\tilde{e}$ and $\tilde{\mu}$ ) that are favorable for the discovery of a signal satisfy the $\mu \rightarrow e \gamma$ bound even for a maximal mixing angle. Thus the LHC has the opportunity of probing mixing angles that are beyond the reach of the current $\mu \rightarrow e \gamma$ limit.

\section{4:1.1 Lepton Flavor Violation due to Slepton Mass Mixing}

To begin consider the lepton-slepton-neutral gaugino vertex with the leptons 
and sleptons in the gauge basis:

$$
\tilde{l}_{a}^{*} \text { gauge } l_{a}^{\text {gauge }} \chi^{0}
$$

where $a=1,2,3$ is a flavor index. Next perform a unitary transformation, $V$, on both $l_{g}$ and $\tilde{l}_{g}$ to go to the mass eigenstate basis for the $l$ 's:

$$
\tilde{l}_{\alpha}^{*} l_{\alpha} \chi^{0}
$$

In this basis the coupling remains diagonal in flavor space (now denoted by $\alpha$ ). In general, however, the slepton and lepton mass matrices are not related so that the same unitary matrix, $V$, may not diagonalize them both. In this general case, the slepton (mass) $)^{2}$ matrix in the basis $\tilde{l}_{\alpha}$ is not diagonal. For example, even if the slepton (mass) $)^{2}$ matrix in the gauge basis $\tilde{l}_{a}^{\text {gauge }}$ of Eqn.(4.1) is diagonal but not $\propto 1$, it will have off-diagonal elements in the basis $\tilde{l}_{\alpha}$ of Eqn.(4.2). So, a further unitary transformation, $W$, is needed to rotate to the slepton mass basis. In this basis the slepton-lepton-gaugino vertex is:

$$
\tilde{l}_{i}^{*} l_{\alpha} W_{i \alpha} \chi^{0}
$$

So, in the mass basis for leptons and sleptons $\left(l_{\alpha}\right.$ and $\left.\tilde{l}_{i}\right)$ a mixing matrix $W \neq 1$ in general appears at the neutral gaugino-lepton-slepton verıex. This means that there is a coupling between, for example, $\tilde{e}$ (in the mass basis), $\mu$ and $\chi^{0}-$ this will be referred to as SUSY lepton flavor violation. The focus of this chapter is the detection of this SUSY lepton flavor violation at the LHC.

The theoretical expectations for $W$ are varied. In models with broken flavor symmetries, it is expected that $W \sim V_{K M}$. In such cases a Cabibbo-like mixing 
angle for the first two generations and a $\Delta m / m$ close to the $\mu \rightarrow e \gamma$ bound is expected [57]. In contrast, in models of gauge-mediated supersymmetry breaking the dominant contribution to the soft masses is universal and it naively appears that there is no interesting flavor physics. There is, however, a subdominant flavor non-universal supergravity contribution. This likely results in large mixing angles [52]. The magnitude of $\Delta m / m$ depends on the supersymmetry breaking scale and while clearly model-dependent, could easily be $\sim \Gamma / m$ or larger, which is needed to give an observable flavor-violating signal at the LHC (this is discussed later in this section).

For simplicity, the case $\because 1-2$ mixing with mixing angle $\theta$ is discussed. In this case there are strong limits on the mixing angle and the $\tilde{e}-\tilde{\mu}$ mass splitting from lepton flavor changing processes. For example, $\mu \rightarrow e \gamma$ gives an important constraint. For degenerate left-handed sleptons, and with the LSP $\left(\chi_{1}^{0}\right)$ approximately bino-like $\left(\tilde{B}^{0}\right)$, the constraint on $\sin 2 \theta_{R}$ and the mass splitting $\Delta m$ between the right-handed sleptons is approximately

$$
\sin 2 \theta_{R}(\Delta m) / m \lesssim 0.01 \times \sqrt{\frac{B R(\mu \rightarrow e \gamma)}{4.9 \times 10^{-11}}}
$$

(A more proper formula is given in section 4.3.2).

Suppose a real selectron is produced in the basis of Eqn.(4.2) (say in association with an electron). Since $\tilde{e}(\alpha=1)$ is not a mass eigenstate, there is a probability that as it propagates it will convert to a $\tilde{\mu}(\alpha=2)$ and hence decay into a $\mu$ 
$[51,52]:$

$$
P\left(\tilde{e}_{\alpha=1} \rightarrow \mu \chi_{1}^{0}\right)=2 \sin ^{2} \theta \cos ^{2} \theta x
$$

where $x=(\Delta m)^{2} /\left((\Delta m)^{2}+\Gamma^{2}\right)$ is the quantum interference factor and assuming $B R\left(\tilde{l} \rightarrow l \chi_{1}^{0}\right)=1$. Here $\Gamma$ is the decay width of the slepton. Note that for $\Delta m \gtrsim \Gamma$ the interference effect can be neglected so that $x \sim 1$. In this case the oscillation probability can be large. Typically, $\Gamma \sim \alpha_{\text {em }} m \sim 0.01 m$ so that $x \sim 1$ if $(\Delta m) / m \gtrsim 0.01$. This is close to the upper bound from the $\mu \rightarrow e \gamma$ limit, so there could be a suppression due to either $x$ or $\sin \theta[51,52]$. It is possible, however, that for a specific SUSY spectrum the decay width could be much smaller than this naive estimate, allowing for a larger range of $\Delta m / m$ consistent with the rare decay limit (even for large mixing angles) and $x \sim 1$ so that the oscillation signal is not suppressed ${ }^{1}$.

Similarly, a neutralino can decay into $e^{+} \mu^{-}$or $e^{-} \mu^{+}$through an intermediate slepton:

$$
\chi_{2}^{0} \rightarrow \tilde{l}^{+} l^{-}, \tilde{l}^{-} l^{+} \rightarrow l^{+} l^{-} \chi_{1}^{0}
$$

Using Eqn.(4.5) the rate for a flavor violating decay is

$$
B R\left(\chi_{2}^{0} \rightarrow e^{+} \mu^{-} \chi_{1}^{0}\right)=2 \sin ^{2} \theta \cos ^{2} \theta x \times B R\left(\chi_{2}^{0} \rightarrow \tilde{e}^{-} e^{+}, \tilde{\mu}^{+} \mu^{-}\right)
$$

Here to simplify notation $B R\left(\chi_{2}^{0} \rightarrow \tilde{e}^{-} e^{+}, \tilde{\mu}^{+} \mu^{-}\right) \equiv B R\left(\chi_{2}^{0} \rightarrow \tilde{e}^{-} e^{+}\right)+B R\left(\chi_{2}^{0} \rightarrow\right.$ $\left.\tilde{\mu}^{+} \mu^{-}\right)$. This notation will be used throughout the chapter. Also, the BR on the right-side of Eqn.(4.7) is in the absence of any mixing. In the case of interest here

\footnotetext{
${ }^{1}$ In fact, this occurs at the LHC Point discussed in section 4.3.2.
} 
of small mass splittings, $\Delta m \ll m$, the neutralino decay rate into selectrons or smuons are equal in the absence of any mixing. Next, in the absence of mixing,

$$
B R\left(\chi_{2}^{0} \rightarrow e^{+} e^{-} \chi_{1}^{0}\right)=2 B R\left(\chi_{2}^{0} \rightarrow \tilde{e}^{+} e^{-}\right)
$$

The factor of two occurs since $\chi_{2}^{0}$ may decay to $\tilde{e}$ 's of both charges. This result and Eqn.(4.7) relates the flavor-violating and flavor-conserving decays:

$$
B R\left(\chi_{2}^{0} \rightarrow e^{+} \mu^{-} \chi_{1}^{0}\right)=2 \sin ^{2} \theta \cos ^{2} \theta x \times B R\left(\chi_{2}^{0} \rightarrow l^{+} l^{-} \chi_{1}^{0}\right)
$$

where the $\mathrm{BR}$ on the right-side of the above equation is in the absence of mixing. Here $l$ is either $e$ or $\mu$. This result applies for $\chi_{2}^{0}$ decays to real sleptons, i.e., for $m_{\chi_{2}^{0}}>m_{\tilde{l}}$. For $m_{\chi_{2}^{0}}<\dot{m}_{\tilde{l}}$, there is an additional suppression of $(\Delta m) / m$ in the decay amplitude due to the supersymmetric analog of the Glashow-IliopoulosMaiani (GIM) cancellation as in the case of $\mu \rightarrow e \gamma$, resulting in negligible $e \mu$ signal. So an observable $e \mu$ signal requires the production of real sleptons ${ }^{2}$.

\subsection{Slepton Production by Drell-Yan Process}

One way to produce sleptons at a hadron collider is through the Drell-Yan process:

$$
p p(\text { or } \bar{p}) \stackrel{\gamma, Z}{\rightarrow} \tilde{l}^{*} \tilde{l} \rightarrow l^{+} l^{-} \chi_{1}^{0} \chi_{1}^{0}
$$

Thus the production of sleptons is identified by events with no jets, 2 hard isolated leptons and $\not \not_{T}$, assuming that $\chi_{1}^{0}$ is stable or decays outside the detector. These events will be referred to as "flavor conserving" dilepton events.

\footnotetext{
${ }^{2}$ Alignment models with $\Delta m \sim m$ are not considered here since $\sin \theta \sim O\left(10^{-2}\right)$.
} 
There is a SM background to the signal from $W^{+} W^{-}$and $\bar{t} t$ production. These backgrounds are known, in principle. In [58] a set of kinematic cuts on the leptons, as well as a jet-veto, are found which sufficiently reduce these backgrounds. These cuts reduce the signal as well - of course, the reduction is much more for the background.

There is also a SUSY background from $p p \rightarrow \chi^{+} \chi^{-} \rightarrow W^{+} W^{-} \chi_{1}^{0} \chi_{1}^{0}$. This background depends on the model-dependent $\chi^{+} \chi^{-}$production cross section. But, for supergravity motivated parameter choices with $m_{\tilde{q}} \approx m_{\tilde{g}}$, this background can be sufficiently reduced by using the same cuts used to remove the SM background [58]. For example, from the analysis of [58] (see Table III of [58]) with $10(\mathrm{fb})^{-1}$ and for a slepton mass $\sim 100 \mathrm{GeV}$ there are $\sim 20$ signal events with no background events remaining after the cuts.

Actually, a clever method [54] for detecting the sleptons is to form the asymme$\operatorname{try} A_{F}=N\left(e^{+} e^{-}+\mu^{+} \mu^{-}\right)-N\left(e^{+} \mu^{-}+e^{-} \mu^{+}\right)$. The background does not contribute to $A_{F}$, so a non-zero value would provide evidence for slepton production.

In the lepton flavor mixing case the pair production of sleptons will produce $e \mu$ events with $\not p_{T}$ - these events will be referred to as "flavor violating" dilepton events. The background to this signal is from the same sources as for the flavor conserving dilepton signal (with the same rate) as well as from $\tilde{\tau} \tilde{\tau}^{*}$ production followed by leptonic decays of $\tau$ s.

The detection of SUSY lepton flavor violation using the above flavor violating dilepton events for the CMS detector at the LHC was studied in references [53, 54] 
for the case of maximal mixing $(\theta=\pi / 4)$. With the mixing angle being maximal, the flavor violating dilepton signal rate is high; see Eqn.(4.5) (assuming $x \sim 1$ ). In fact, the number of flavor conserving and flavor violating events from slepton production in this case are the same and each is equal to one half the signal in the zero mixing case so that $A_{F} \approx 0$ (unlike the case of zero or non-maximal mixing). In the case where the production cross-sections for staus $(\tilde{\tau})$ and the lightest charginos are comparable to that of the sleptons, the production rate for the SUSY background to $e \mu$ events is $\sim 4 \%$ of the total flavor conserving signal (in the absence of mixing). ${ }^{3}$ Thus, the chargino and stau backgrounds are much smaller. The high signal and low SUSY background rate (compared to the signal) for maximal mixing enables detection of a $5 \sigma$ flavor violating signal for sleptons masses up to $250 \mathrm{GeV}$ and LSP masses $m_{\chi_{1}^{0}}<0.4 m_{\tilde{e}_{R}}$ with an integrated luminosity of $100 \mathrm{fb}^{-1}$.

There are some objections to the generality of this result, though. A more general spectrum could result in a larger chargino or stau background. For example, there is no reason to expect the chargino production cross-section to be related to the slepton production cross-section. However, as mentioned above, the kinematics of slepton production and decay are different enough from that of the chargino background that an appropriate set of kinematic cuts could distinguish

\footnotetext{
${ }^{3}$ Here, it is assumed that $B R\left(\chi^{+} \rightarrow W^{+} \chi_{1}^{0}\right) \approx 100 \%$ so that the leptonic BRs of $\chi^{+}$are the same as for $W$. If the left-handed sleptons are lighter than $\chi^{+}$, then the leptonic BRs of $\chi^{+}$may be enhanced substantially, in turn increasing the SUSY background.
} 
the two, at least for supergravity motivated parameter choices with comparable squark and gluino masses [58]. Next, the stau background is sensitive to the stau mass, which is likely to differ from the selectron and smuon masses ${ }^{4}$. The stau background has softer leptons, so a cut on the $p_{T}$ of the leptons may help distinguish this background from the signal. The success of this may require large statistics and knowledge of the stau production cross-section. Thus, in general, the SUSY background may not be small.

Next, detection of flavor violation for smaller mixing angles is discussed. Since the signal is $\propto \sin ^{2} \theta$, it is significantly smaller for say Cabibbo-like mixing angles. In this case, it is crucial to know the SUSY background more precisely since it is comparable to the signal (assuming similar cross sections for sleptons and charginos). While the quantity $A_{F}$ ( $>0$ for non-maximal mixing) is, up to statistical fluctuations, background-free as far as slepton detection is concerned, it is not useful for providing evidence for slepton flavor violation since the chargino background would need to be determined first. This is because the deviations in the values of $A_{F}$ and $N(e \mu)$ from the SM for a non-zero mixing angle could be reproduced, in the case of zero mixing angle, with a lower slcpton production cross-section and a higher chargino production cross-section.

Even if the SUSY background can be reduced sufficiently by an appropriate set of cuts, since the signal is suppressed by the small mixing angle (there will

\footnotetext{
${ }^{4}$ The rare decays $\tau \rightarrow e \gamma, \tau \rightarrow \mu \gamma$ and $\mu \rightarrow e \gamma$ allow for $O(1)$ splitting between the third and first two generation scalars for $C K M$-like mixing angles.
} 
also be a reduction of the signal due to these cuts), it may not possible to probe Cabibbo-like mixing angles. For example, in the case of no mixing, Table 4 of reference [54] gives 195 dilepton signal events for the set of cuts labeled 1 with $L=10 \mathrm{fb}^{-1}$ and a slepton mass of $100 \mathrm{GeV}$. The number of signal events in the case of mixing for $L=100 \mathrm{fb}^{-1}$ is then $1950 \times 2 \times \sin ^{2} \theta \cos ^{2} \theta$ (assuming $x \sim 1$ ). The SM background from $W W$ production is 9920 for the same set of cuts. Thus a $5 \sigma$ signal (requiring $S / \sqrt{B}>5$ ) is possible only for $\sin \theta \gtrsim 0.4$. Since this signal was obtained for a $24 \mathrm{GeV}$ LSP, only larger angles will be probed for larger LSP masses (since the leptons will be softer in that case). For sleptons heavier than $100 \mathrm{GeV}$ the prospects for detecting small mixing angles are clearly worse.

Thus, in the situation where the SUSY background is known to be small, e.g. if an appropriate set of cuts for a more general spectrum can separate the chargino background from the signal, then the flavor violating dilepton events from DrellYan production of sleptons is a promising signal for the detection of flavor violation in the case of large mixing angles. Otherwise, it is important to look for other discovery channels for slepton flavor violation.

\subsection{Slepton Production in Cascade Decays}

The other way to produce sleptons is through the cascade decays of gluinos and squarks. At the LHC, the production cross sections of squarks and gluinos are much larger than the Drell-Yan production of sleptons, neutralinos, and charginos. So, a larger production of sleptons (if they are light) is expected in the cascade 
decays than from direct Drell-Yan production. In a generic SUSY event, the production of two real (or virtual from gluino decay) squarks will be followed by their cascade decays ultimately to the LSP through intermediate electroweak sparticles (sleptons, charginos, neutralinos). Assuming for simplicity that the spectrum is gaugino-like, i.e., $\chi_{2}^{0} \approx \tilde{W}_{3}, \chi_{1}^{+} \approx \tilde{W}^{+}$and $\chi_{1}^{0} \approx \tilde{B}$, the following squark decays are obtained:

$$
\begin{aligned}
B R\left(\tilde{q}_{R} \rightarrow q \chi_{1}^{0}\right) & \approx 1, \\
B R\left(\tilde{q}_{L} \rightarrow q \chi_{2}^{0}\right) & \approx \frac{1}{3}, \\
B R\left(\tilde{q}_{L} \rightarrow q^{\prime} \chi_{1}^{+,-}\right) & \approx \frac{2}{3} .
\end{aligned}
$$

Thus, a typical SUSY event is:

$$
\begin{aligned}
p p & \rightarrow \tilde{g} \tilde{g}, \tilde{g} \tilde{q} \rightarrow \tilde{q} \tilde{q} \\
& \rightarrow \chi_{E W} \chi_{E W}^{\prime}+X
\end{aligned}
$$

with $\chi_{E W}, \chi_{E W}^{\prime}$ one of $\chi_{1,2}^{0}, \chi_{1}^{+,-}$.

\subsubsection{Dilepton Events}

If one of the squarks decays to $\chi_{2}^{0}$ followed by the decay of $\chi_{2}^{0}$ to a slepton (if $B R\left(\chi_{2}^{0} \rightarrow \tilde{l} l\right)$ is significant) a large number of $e \mu$ events in the presence of lepton flavor mixing (see Eqns.(4.6) and (4.9)) is obtained. These events also have at least 2 high $p_{T}$ jets and large $\not p_{T}$.

There is no background from $W^{+} W^{-}$production since this background contains no hard jets (assuming jet detection is good). There is a background from 
$t \bar{t}$ production followed by leptonic decays of the $W$ 's from the top quarks. This can be reduced by rejecting events with $b$-jets or using a high $\not p_{T}$ cut.

There is a SUSY background from the decays of both squarks to charginos, followed by chargino decays to $W^{+}, W^{-}$or $\tilde{l}, \tilde{l}^{*}$. This background is distinguishable from the signal though. The invariant mass distribution of the 2 leptons from the $\chi_{2}^{0}$ decay has a sharp edge (which is a function of the neutralino and slepton masses) $[55,56]$ unlike the case of the 2 leptons from $\chi^{+} \chi^{-}$decays. Also, the angle between the 2 leptons from the decay of $\chi_{2}^{0}$ is likely to be smaller than in the case of 2 leptons from $\chi^{+}$and $\chi^{-}$. Such kinematic cuts on the invariant mass of the dileptons and the angle etween them easily reduce the number of background events sufficiently if we are interested in detecting flavor conserving dileptons from $\chi_{2}^{0}$ decays.

But, in the case of the flavor violating dilepton events, (as in section 4.2) since the signal is suppressed by the mixing angle (while the background is the same), the number of background events that survive (relative to the signal) after cuts depends crucially on the model-dependent cross sections for producing $\chi^{+} \chi^{-}$ vs. $\chi_{2}^{0}{ }^{5}$. So in general it is difficult to be sure that the cuts have reduced the

\footnotetext{
${ }^{5}$ For example, the ratio of the number of events with $\chi^{+} \chi^{-}$to those with (at least) one $\chi_{2}^{0}$ is larger for $s$-channel $\tilde{q} \tilde{q}^{*}$ production than for gluino pair production which is seen as follows. For the $\tilde{g} \tilde{g}$ case, the probability of getting two $\tilde{q}_{L}$ is $1 / 4$ compared to a probability of $3 / 4$ for getting at least one $\tilde{q}_{L}$ whereas for $s$-channel $\tilde{q} \tilde{q}^{*}$ production the probabilities are the same. Same sign chargino events are also obtained from $\tilde{g} \tilde{g}$ production whereas $s$-channel $\tilde{q} \tilde{q}^{*}$ production can give only opposite-sign chargino pairs. Thus, if the $s$-channel $\tilde{q} \tilde{q}^{*}$ production is larger, the number
} 
background sufficiently. ${ }^{6}$

In the circumstance that $\chi^{+} \chi^{-}$are dominantly produced from $\tilde{g} \tilde{g}$ cascade decays, the $\chi^{+} \chi^{-}$flavor violating background can be estimated as follows. An equal number of same sign and opposite-sign chargino pairs are expected since $\tilde{g}$ is a Majorana particle. The same-sign chargino pairs produce same-sign dileptons so that the opposite-sign chargino $e \mu$ background can be estimated from the number of same-sign $e e$ and $\mu \mu$ events. Unfortunately, in the more general case the $\chi^{+} \chi^{+}$ and $\chi^{+} \chi^{-}$production cross sections are not related since the chargino pairs do not always come from gluino pair decays. For example, $p p \rightarrow \tilde{q}_{L} \tilde{q}_{L}^{*}$ can lead to $\chi^{+} \chi^{-}$, but not to $\chi^{+} \chi^{+}$.

It might be possible to estimate the $\chi^{+} \chi^{-}$background by analyzing the (observed) (signal + background) distribution of the invariant mass of the flavor violating dileptons [59]. As mentioned earlier, the dilepton invariant mass distribution for $\chi_{2}^{0}$ decay has a sharp edge unlike the case of the background. The position of this edge (denoted by $M_{l l}$ ) can be easily found by looking at the distribution of the invariant mass of flavor conserving dileptons (where the $\chi^{+} \chi^{-}$ of $\chi^{+} \chi^{-}$events relative to $\chi_{2}^{0}$ events increases.

${ }^{6}$ There is also a SUSY background from $\chi_{2}^{0}$ decays to $\tilde{\tau} \tau$ followed by leptonic decays of the $\tau$ 's. A cut on the dilepton invariant mass can reduce this: the leptons from the $\tau$ decays are softer than those from the $\tilde{e} / \tilde{\mu} / \chi_{2}^{0}$ decays and so have a smaller invariant mass. But, since, in general, $B R\left(\chi_{2}^{0} \rightarrow \tilde{\tau} \tau\right)$ is not related to $B R\left(\chi_{2}^{0} \rightarrow \tilde{e} e\right)$, as for the chargino background, we cannot be sure that the $\tilde{\tau} \tau$ background has been sufficiently reduced (by the cuts) since this background is unknown. 
background is very small) $[55,56]$. In the case of flavor violating decays, the existence of an edge in the (observed) opposite-flavor dilepton distribution (e.g. e $\mu$ ) at $M_{l l}$ would then be an indication of flavor violation. However, since the flavor violating dilepton signal is suppressed by (small) mixing relative to the flavor conserving dilepton signal (whereas the $\chi^{+} \chi^{-}$background is the same for both kinds of dileptons), the edge at $M_{l l}$ in the opposite flavor dilepton case might not be as sharp as for the same flavor dilepton case - this depends on the model-dependent cross sections for producing $\chi^{+} \chi^{-}$vs. $\chi_{2}^{0}$.

Next, in the distribution of the invariant mass of the flavor violating dileptons, the events beyond $M_{l l}$ (this value can be obtained from the same flavor dilepton distribution if the edge is not so sharp in the opposite-flavor dilepton distribution) are mostly from the $\chi^{+} \chi^{-}$background [59]. Extrapolating (assuming say a flat distribution for the $\chi^{+} \chi^{-}$background) from the data in this region, the $\chi^{+} \chi^{-}$ background in the region with invariant mass less than $M_{l l}$ can be estimated. An excess of $e \mu$ events (with invariant mass between zero and $M_{l l}$ ) over this estimate will be a signal for flavor violation. ${ }^{7}$. This extrapolation may not be reliable for invariant masses much smaller than $M_{l l}$ since the distribution of the $\chi^{+} \chi^{-}$ background in this region is not known. A detailed simulation is required to know this distribution (it is known only that it does not have an edge at $M_{l l}$ ). Near $M_{l l}$ the extrapolation should be more reliable and that is the region where the signal

\footnotetext{
${ }^{7}$ The invariant mass of the leptons from the $\tilde{\tau} \tau$ decays (from $\chi_{2}^{0}$ ) is less than $M_{l l}$ and so this background cannot be estimated this way.
} 
is peaked (since the flavor violating dileptons from $\chi_{2}^{0}$ decay also have a sharp edge at $M_{l l}$ ). An excess in this region (rather than the whole region between zero mass and $M_{l l}$ ) might thus be a better signal for flavor violation [59] - as mentioned earlier, the distribution will have a edge (or a "step") at $M_{l l}$. Also, the $\tilde{\tau} \tau$ background in the region near $M_{l l}$ is negligible since the leptons from these decays are softer [59]. However, statistics are larger if the region from zero mass to $M_{l l}$ is used.

The chargino background can also be eliminated in considering a flavor violating and $C P$ violating dilepton signal [52]. The presence of non-trivial phases in the slepton mixing matrix $W$ breaks $C P$, and results in a non-vanishing asymmetry: $N\left(e^{+} \mu^{-}-e^{-} \mu^{+}\right) \neq 0$. In this case, the $\chi^{+} \chi^{-}$background is not important since it is CP symmetric.

To summarize, if the number of $e \mu$ events (that pass certain cuts) from either Drell-Yan or cascade production is used to detect flavor violation, the SUSY background from $\chi^{+} \chi^{-}$pairs (which passes the same cuts) is difficult to estimate, in general, and may be too large. The possibility of using the observed oppositeflavor dilepton mass distribution (in the case of cascade decays) to estimate the chargino background is interesting, though, and warrants further study [59].

\subsubsection{Events with 4 leptons}

A dramatic flavor violating signal is obtained through the pair production of two $\chi_{2}^{0} \mathrm{~s}$, followed by the decays of both $\chi_{2}^{0} \mathrm{~s}$ to slepton and lepton pairs. Such an 
event contains 4 leptons and occurs if both squarks in a SUSY event decay into $\chi_{2}^{0}$. If one of the $\chi_{2}^{0} \mathrm{~s}$ has a flavor violating decay: $\chi_{2}^{0} \rightarrow \tilde{l} l \rightarrow e \mu$, then events containing $3 e 1 \mu$, or $3 \mu$ 1e will be produced. A typical decay chain is then:

$$
\begin{aligned}
\tilde{q}_{L} \tilde{q}_{L}^{\prime} & \rightarrow \chi_{2}^{0} q+\chi_{2}^{0} q^{\prime} \\
\chi_{2}^{0} & \rightarrow \tilde{l} l \rightarrow e^{+} e^{-} \chi_{1}^{0} \\
\chi_{2}^{0} & \rightarrow \tilde{l}^{\prime} l^{\prime} \rightarrow \mu^{+} e^{-} \chi_{1}^{0} .
\end{aligned}
$$

These events are identified by 4 isolated leptons (with the $3+1$ flavor structure), at least 2 high $p_{T}$ jets, $\not p_{T}$, and concentrating on only those events produced from the decays of first two generation squarks, no $b$-jets. These events will be referred to as "flavor violating" 4 lepton events. The absence of $b$-jets is important in distinguishing the signal from other SUSY and SM backgrounds (see below).

The backgrounds to these events arise from both SM and SUSY sources.

The dominant SM background occurs from $t \bar{t}$ production with semileptonic decays of the $b$ s (or $t \bar{t} \gamma$ production with 2 leptons from $\gamma$ ) and leptonic decays of the $W \mathrm{~s}$. In this case, however, the leptons from $b$ decays will not be isolated (or the invariant mass of 2 of the leptons will be zero in the case of $t \bar{t} \gamma$ ). Also, these events have $2 b$ quarks and can be rejected using $b$-jet veto. Double gauge boson production can give 4 lepton events, but none of these events have the $3+1$ flavor structure. Triple gauge boson production ( $W W Z$ or $W W \gamma$ ) can give events with 4 leptons and the correct flavor asymmetry, but some initial state gluon radiation is needed to give the 2 hard jets. The production cross-section for such events is 
small. Also, events of this kind can also be rejected since the invariant mass of 2 of the leptons will either be zero or $m_{Z}$.

One important obstacle in identifying flavor-violating dilepton events was the potentially large background from $\chi^{+} \chi^{-}$production. In the 4 lepton signal, however, there is no $\chi^{+} \chi^{-}$background from the squark decays since this gives only 2 leptons.

The weak decay $\tilde{q} \rightarrow W \tilde{q}^{\prime}$, if kinematically allowed, can lead to a possible background. For example, the process

$$
\begin{aligned}
\tilde{q}_{L} \tilde{q}_{L}^{\prime} & \rightarrow W^{-} \tilde{q}_{L}^{\prime \prime}+\chi_{1}^{+} q \\
W^{-} & \rightarrow e^{-} \bar{\nu} \\
\tilde{q}_{L}^{\prime \prime} & \rightarrow \chi_{2}^{0} q^{\prime \prime} \rightarrow \mu^{+} \mu^{-}+\cdots \\
\chi_{1}^{+} & \rightarrow \mu^{+} \nu \chi_{1}^{0}
\end{aligned}
$$

is a potential background. For the first two generation squarks, however, the decay $\tilde{q} \rightarrow W \tilde{q}^{\prime}$ is kinematically forbidden. This is because the mass splitting in an electroweak doublet occurs from the electroweak $D$-terms and is less than $m_{W}^{2} / m_{\bar{q}}<m_{W}$. This process is allowed for the top and bottom squarks, but such an event contains $2 b$-jets and this background can be reduced with a $b$-jet veto.

There is a SUSY background to the flavor violating 4 lepton events from production of heavier neutralinos or chargino in the cascade decays of squarks. For example,

$$
\tilde{q}_{L} \tilde{q} \rightarrow \chi_{3}^{0} \chi_{2}^{0}+\cdots,
$$




$$
\begin{aligned}
\chi_{3}^{0} \rightarrow W^{+} \chi^{-} & \rightarrow e \mu+\cdots, \\
\chi_{2}^{0} \rightarrow \tilde{l l} & \rightarrow e e(\text { or } \mu \mu) \chi_{1}^{0} .
\end{aligned}
$$

This background is small in the so-called gaugino-like region. In this region there is very little gaugino-Higgsino mixing. Then, the heavier chargino and the two heaviest neutralinos are dominantly Higgsinos and the two lightest neutralinos and the lighter chargino are mainly gauginos; this turns out to be typical of the SUSY parameter space still allowed by experimental data. Thus, the decays of the first two generation squarks into the heavier neutralinos or chargino are highly suppressed by the first two generation Yukawa couplings, small gaugino-Higgsino mixing, and also by phase space.

Another potentially large background can also occur from the production of the heavier sleptons (say, the left-handed) and/or sneutrinos. Sleptons can decay to $\chi_{2}^{0} l$ and $\tilde{\nu}$ to $\chi_{1}^{ \pm} l$ if kinematically allowed. If the neutralino and chargino decay to leptons, then this decay chain can give 3 (or 2) leptons. With 1 (or 2 ) leptons from another decay of this kind (or some other decay chain), this can mimic the flavor violating 4-lepton signal. If the left-handed sleptons are paired produced through the Drell-Yan mechanism, then these events do not contain any hard jets and may be rejected. Thus, the only source for a background from heavier sleptons is their production in the decays of gluinos and squarks. Such a decay does not occur directly, but only through the decays of gluinos and squarks to the heavier neutralino and chargino. The heavier neutralinos and chargino can then decay to the left-handed sleptons. As argued in the previous paragraph though, in the 
gaugino-like region, the heavier neutralinos/chargino are dominantly Higgsinos so that their decays to the sleptons are suppressed by the lepton Yukawa couplings and small gaugino/Higgsino mass mixing angles. So this background is negligible.

However, top squarks (and bottom squarks for large $\tan \beta$ ) will have significant decay branching fractions into heavier neutralinos or chargino even if they are purely Higgsinos since the third generation Yukawa couplings (and hence couplings of the squarks to Higgsinos) are large. Further, as mentioned earlier, $W$ s may be produced in the direct decay of stops or sbottoms. Also, top quarks from stop or sbottom decays produce $W \mathrm{~s}$. Both of these processes give additional isolated leptons. This leads to a potential background even if stops or sbottoms decay only to the lighter chargino and neutralinos. For example, the following decay chain is a possible background:

$$
\begin{aligned}
\tilde{t}^{*} & \rightarrow b \chi^{-}+t \chi_{2}^{0}, \\
t & \rightarrow W^{+} b \rightarrow e^{+} b+\cdots, \\
\chi^{-} & \rightarrow W^{-} \chi_{1}^{0} \rightarrow \mu^{-}+\cdots, \\
\chi_{2}^{0} & \rightarrow e^{+} e^{-} \chi_{1}^{0} .
\end{aligned}
$$

These backgrounds to flavor violating 4 lepton events can be reduced by rejecting any 4 lepton event that contains at least one $1 b$-jet. Note that the top or bottom squark background has at least $2 b$ quarks. The efficiency for rejecting this background is discussed in a later section where a specific spectrum is considered.

There is also an important SUSY background from decays of taus and staus 
produced from the decays of two $\chi_{2}^{0} \mathrm{~s}$. That is,

$$
\begin{aligned}
& \chi_{2}^{0} \rightarrow \tilde{\tau} \tau \rightarrow e \mu \chi_{1}^{0}+\ldots, \\
& \chi_{2}^{0} \rightarrow \tilde{l} l \rightarrow e e(\text { or } \mu \mu) \chi_{1}^{0} .
\end{aligned}
$$

This background can be estimated/measured as follows. In the above decay chain, if one $\tau$ decays hadronically instead of leptonically, the result is $3 e 1 \tau$-jet events. If a lower bound on the $\tau$-jet detection efficiency is known, an upper bound on the number of $3 e 1 \mu$ events coming from $\tau$ decays is obtained by using the number of $3 e 1 \tau$-jet events. An excess of $3 e 1 \mu$ events over this background is a signature of lepton flavor violation.

Lastly, the following $\chi_{2}^{0}$ decay chains can also give flavor violating dileptons:

$$
\begin{aligned}
\chi_{2}^{0} & \rightarrow h \text { (or) } Z \chi_{1}^{0} \\
h \text { (or) } Z & \rightarrow \tau \tau \rightarrow e \mu .
\end{aligned}
$$

In combination with another $\chi_{2}^{0}$ decay to $e e$ or $\mu \mu$, these decay chains can give flavor violating 4-lepton events. In the gaugino region, the decay $\chi_{2}^{0} \rightarrow Z \chi_{1}^{0}$ is suppressed since there is no vertex with $Z$ and 2 neutral gauginos. In any case, an effective $B R\left(\chi_{2}^{0} \rightarrow \tau \tau\right)$ can be defined to include these two decay chains in addition to the $\chi_{2}^{0} \rightarrow \tilde{\tau} \tau$ decay. It will be shown in section 4.3 .2 that this (in general unknown) BR does not affect the estimate of the (effective) $\tau$ background obtained by using the $3 e 1 \tau$-jet events.

\section{A quick estimate of number of 4 lepton events}


A typical value for the total SUSY production cross section (gluinos and squarks) at the LHC is:

$$
\sigma_{S U S Y} \sim 10 \frac{\pi \alpha_{S}^{2}}{\hat{s}} \sim 100 \mathrm{pb}
$$

with $\sqrt{\hat{s}} \sim 1 \mathrm{TeV}, \alpha_{S} \sim 0.1$ and summed over colors and generations (the factor of 10). Assuming that the probability to get a $\tilde{q}_{L}$ is $1 / 2$ and $B R\left(\tilde{q}_{L} \rightarrow \chi_{2}^{0} q\right)=1 / 3$, this gives

$$
\sigma_{\chi_{2}^{0} \chi_{2}^{0}} \sim \sigma_{S U S Y}\left(\frac{1}{2}\right)^{2}\left(\frac{1}{3}\right)^{2} \sim 3 \mathrm{pb}
$$

If $B R\left(\chi_{2}^{0} \rightarrow \chi_{1}^{0} l^{+} l^{-}\right) \sim 0.16$ (for each of $\left.l=e, \mu\right)$ and for $\sim$ one year of running at low luminosity which gives an integrated luminosity of $L \sim 10(\mathrm{fb})^{-1}$, the expected number of events is :

$$
\begin{aligned}
N\left(\chi_{2}^{0} \chi_{2}^{0}\right) & \sim 30,000, \\
N(4 l \text { where } l=e, \mu)= & (2 \times 0.16)^{2} N\left(\chi_{2}^{0} \chi_{2}^{0}\right) \sim 3300, \\
N\left(3 l+l^{\prime}\right)= & 4 \sin ^{2} \theta \cos ^{2} \theta x N(4 l) \sim 550,
\end{aligned}
$$

for $\sin \theta \approx 0.2$ and $x \sim 1$. To be clear,

$$
S_{F V} \equiv N\left(3 l+l^{\prime}\right)=\left(N\left(e^{+} \mu^{-} \mu^{+} \mu^{-}\right)+(+\leftrightarrow-)\right)+(\mu \leftrightarrow e),
$$

and

$$
N(4 l)=N\left(e^{+} e^{-} e^{+} e^{-}\right)+N\left(e^{+} e^{-} \mu^{+} \mu^{-}\right)+N\left(\mu^{+} \mu^{-} \mu^{+} \mu^{-}\right)+S_{F V} .
$$

In the next section this definition for $N(4 l)$ is trivially extended to include leptons produced by the decay of $\tau \mathrm{s}$. Thus, typically, a large number of 4 lepton flavor 
violating events is expected from the cascade decays of squarks ${ }^{8}$.

\section{Detailed estimates at Point 5 (Point $A$ ) of LHC studies}

One Point of the LHC supersymmetry studies $[55,56]$ contains a spectrum that is favorable for the detection of a flavor-violating 4 lepton signal. The minimal supergravity input parameters for this point are:

$$
\begin{array}{cc}
m_{0}=100 \mathrm{GeV}, \quad M_{1 / 2}=300 \mathrm{GeV}, \quad & A_{0}=300 \mathrm{GeV}, \\
\tan \beta=2.1, \quad \operatorname{sgn}(\mu)=+, \quad & m_{\text {top }}=170 \mathrm{GeV} .
\end{array}
$$

Renormalization group evolution of these input parameters to the weak scale results in a mass spectrum which is given in Table 4.1. Note that $m_{\chi_{2}^{0}} \approx 230 \mathrm{GeV}>$ $m_{\tilde{l}_{R}} \approx 160 \mathrm{GeV}$ so that the decay of $\chi_{2}^{0}$ into real sleptons is allowed.

The production cross-section for SUSY particles is presented in Table 4.2, and is dominated by $\tilde{g} \tilde{q}$ production. In total $\sigma_{S U S Y} \approx 16 \mathrm{pb}$. To estimate the number of signal and background events, the branching fractions of the sparticles are needed. These are given in Table 4.3. Note that at this Point $B R\left(\chi_{2}^{0} \rightarrow \tilde{l}_{i} l_{i}\right) \sim 0.12$ and is reduced due to the large branching fraction $B R\left(\chi_{2}^{0} \rightarrow h \chi_{1}^{0}\right)$. This gives from

\footnotetext{
${ }^{8}$ Both $\chi_{2}^{0} \mathrm{~S}$ decaying to flavor violating dileptons gives $\left(e^{+} \mu^{-}\right)\left(e^{+} \mu^{-}\right)$and $\left(e^{+} \mu^{-}\right)\left(\mu^{+} e^{-}\right)$ events. The latter cannot be distinguished from the events where one $\chi_{2}^{0}$ decays to $e^{+} e^{-}$and the other to $\mu^{+} \mu^{-}$. The former events can also be used as a signal of flavor violation, but the number of these events is expected to be very small since they require both $\chi_{2}^{0} \mathrm{~s}$ to decay into flavor violating dileptons. For simplicity these events were not included in Eqn.(4.23).
} 


\begin{tabular}{cccccccc}
\hline$\tilde{g}$ & 770 & $\tilde{q}_{L}$ & 685 & $\tilde{q}_{R}$ & 660 & $h$ & 100 \\
\hline$\tilde{t}_{1}$ & 500 & $\tilde{t}_{2}$ & 715 & $\tilde{b}_{1}$ & 635 & $\tilde{b}_{2}$ & 660 \\
\hline$\tilde{l}_{L}$ & 240 & $\tilde{l}_{R}$ & 160 & $\chi_{1}^{ \pm}$ & 230 & $\chi_{2}^{ \pm}$ & 500 \\
\hline$\chi_{1}^{0}$ & 120 & $\chi_{2}^{0}$ & 230 & $\chi_{3}^{0}$ & 480 & $\chi_{4}^{0}$ & 505 \\
\hline
\end{tabular}

Table 4.1: Mass spectrum in $\operatorname{GeV}$ at LHC Point $[55,56]$. Here $\tilde{q}=\tilde{u}, \tilde{d}, \tilde{c}, \tilde{s}$, and $\tilde{l}=\tilde{e}, \tilde{\mu}, \tilde{\tau}$.

\begin{tabular}{ccccccc}
\hline$\tilde{g} \tilde{g}$ & 1750 & $\tilde{g} \tilde{q}, \tilde{g} \tilde{q}^{*}$ & 8300 & $\tilde{q} \tilde{q}^{*}$ & 2380 \\
\hline$\tilde{q} \tilde{q}^{\prime}$ & 2820 & $\tilde{b} \tilde{b}^{*}$ & 300 & $\tilde{t} \tilde{t}^{*}$ & 700 \\
\hline
\end{tabular}

Table 4.2: The production cross-sections in fb for different SUSY particles at the LHC Point $[55,56]$. Here all flavors $\tilde{q}_{H}=\tilde{u}, \tilde{d}, \tilde{c}, \tilde{s}$ and $H=L, R$ are summed over. 


\begin{tabular}{cccccc}
\hline$\tilde{g} \rightarrow q \tilde{q}_{L}$ & 30 & $\tilde{g} \rightarrow q \tilde{q}_{R}$ & 30 & $\tilde{g} \rightarrow \tilde{t}_{1} t$ & 14 \\
\hline$\tilde{g} \rightarrow \tilde{b}_{L} b$ & 15 & $\tilde{g} \rightarrow \tilde{b}_{R} b$ & 10 & $\tilde{t}_{2} \rightarrow Z_{0} \tilde{t}_{1}$ & 26 \\
\hline$\tilde{t}_{2} \rightarrow \chi_{4}^{0} t$ & 21 & $\tilde{t}_{2} \rightarrow \chi_{2}^{ \pm} b$ & 18 & $\tilde{t}_{2}\left(\tilde{t}_{1}\right) \rightarrow \chi_{1}^{ \pm} b$ & $15(63)$ \\
\hline$\tilde{t}_{2}\left(\tilde{t}_{1}\right) \rightarrow \chi_{2}^{0} t$ & $8(17)$ & $\tilde{t}_{2} \rightarrow \chi_{3}^{0} t$ & 6 & $\tilde{t}_{2}\left(\tilde{t}_{1}\right) \rightarrow \chi_{1}^{0} t$ & $6(20)$ \\
\hline$\tilde{q}_{L} \rightarrow q \chi_{2}^{0}$ & 32 & $\tilde{q}_{L} \rightarrow q \chi_{1}^{ \pm}$ & 64 & $\tilde{q}_{L} \rightarrow q \chi_{1}^{0}$ & 1.5 \\
\hline$\tilde{q}_{L} \rightarrow q \chi_{2}^{ \pm}$ & 1.5 & $\tilde{q}_{L} \rightarrow q \chi_{4}^{0}$ & 1 & $\tilde{q}_{R} \rightarrow q \chi_{1}^{0}$ & 99 \\
\hline$\chi_{2}^{0} \rightarrow \tilde{l}_{R} l$ & 36 & $\chi_{2}^{0} \rightarrow h \chi_{1}^{0}$ & 63 & $\tilde{l}_{R} \rightarrow l \chi_{1}^{0}$ & 100 \\
\hline$\tilde{l}_{L} \rightarrow \chi_{1}^{0} e$ & 90 & $\chi_{1}^{+} \rightarrow W^{+} \chi_{1}^{0}$ & 98 & $h \rightarrow \tau \tau$ & 5 \\
\hline
\end{tabular}

Table 4.3: Branching fractions (in percent) for sparticles at LHC Point [56]. Here $\tilde{q}=\tilde{u}, \tilde{d}, \tilde{c}, \tilde{s}$, and $\tilde{l}=\tilde{e}, \tilde{\mu}, \tilde{\tau}$.

decays of first two generation squarks the number of $\chi_{2}^{0}$ pairs produced:

$$
N\left(\chi_{2}^{0} \chi_{2}^{0}\right)=(0.32)^{2} \times\left(\sigma_{\tilde{g} \tilde{g}} \times(0.3)^{2}+\frac{1}{2} \sigma_{\tilde{g} \tilde{q}} \times 0.3+\frac{1}{4} \sigma_{\tilde{q} \tilde{q}^{\prime}}+\frac{1}{2} \sigma_{\tilde{q}^{*} \tilde{q}}\right) L \approx 3400
$$

(The factors of $1 / 2$ and $1 / 4$ are easy to understand: $1 / 2$ of all $\tilde{q} \tilde{q}^{*}$ produced from schannel gluon and 4-point contact interaction ${ }^{9}$, and $1 / 4$ of all $\tilde{q} \tilde{q}^{\prime}$ s produced (from t-channel gluino exchange) are left-handed pairs.) This is for one year of running at low luminosity $\left(L=10 \mathrm{fb}^{-1}\right)$ and for one detector. Hereafter estimates of event numbers will use this integrated luminosity. A realistic detection efficiency of $90 \%$ for single $e, \mu$, and $90 \%$ for the single-prong decay $\tau \rightarrow \pi \nu(B R \approx 0.11)$ will be used. These are needed to determine the number of 4-lepton and 3-lepton $+\tau$-jet

\footnotetext{
${ }^{9}$ It is assumed that all of the $\tilde{q} \tilde{q}^{*}$ production is by this channel. This is reasonable since most of the hard collisions at LHC energies are likely to be gluon-gluon.
} 
events that are detected. Later, a comment on a more realistic $\tau$-jet detection will be made.

Next the 4-lepton signal and background are estimated.

Due to the decay chain

$$
\begin{gathered}
\chi_{2}^{0} \rightarrow h \chi_{1}^{0} \\
h \rightarrow \tau \tau
\end{gathered}
$$

the effective $B R\left(\chi_{2}^{0} \rightarrow \tau \tau \chi_{1}^{0}\right) \equiv R_{\tau}$ is

$$
\begin{aligned}
R_{\tau} & =B R\left(\chi_{2}^{0} \rightarrow \tilde{\tau}_{R} \tau\right)+B R\left(\chi_{2}^{0} \rightarrow h \chi_{1}^{0}\right) \times B R(h \rightarrow \tau \tau) \\
& =0.15
\end{aligned}
$$

Using the above BR and $B R\left(\chi_{2}^{0} \rightarrow \tilde{l}_{R} l\right)=0.12$ for each of $l=e, \mu$ and $B R(\tau \rightarrow e \nu) \approx B R(\tau \rightarrow \mu \nu) \approx 1 / 2 \times 0.35$,

$$
\begin{aligned}
B R\left(\chi_{2}^{0} \rightarrow e e \chi_{1}^{0}, \mu \mu \chi_{1}^{0}\right)= & 2 \times 0.12 \times\left(1-2 \sin ^{2} \theta \cos ^{2} \theta x\right) \\
& +R_{\tau} \times(0.35)^{2} \times \frac{1}{2} \\
B R\left(\chi_{2}^{0} \rightarrow e \mu \chi_{1}^{0}\right)= & 2 \times 0.12 \times 2 \sin ^{2} \theta \cos ^{2} \theta x \\
& +R_{\tau} \times(0.35)^{2} \times \frac{1}{2}
\end{aligned}
$$

where the first terms in each equation are from decays of $\tilde{e}$ and $\tilde{\mu}$ and the second terms are from $\tau$ decays.

Then, the total number of 4-lepton events expected from $\chi_{2}^{0}$ pair decays (including detection efficiencies, but parameterizing the acceptance cut as $\varepsilon_{C U T}-$ see 
later $\left.{ }^{10}\right)$ is

$$
\begin{aligned}
N(4 l) & =N\left(\chi_{2}^{0} \chi_{2}^{0}\right) \times\left(B R\left(\chi_{2}^{0} \rightarrow e e, \mu \mu, e \mu\right)\right)^{2}(0.9)^{4} \times \varepsilon_{C U T} \\
& =3400 \times\left(0.24+R_{\tau} \times(0.35)^{2}\right)^{2} \times(0.9)^{4} \times \varepsilon_{C U T} \\
& \approx 149 \times \varepsilon_{C U T} .
\end{aligned}
$$

To get $3 e 1 \mu+3 \mu 1 e$ events, one $\chi_{2}^{0}$ has to decay into $e e / \mu \mu$ and the other to $e \mu$. Thus, the number of $3 e 1 \mu+3 \mu$ le events from flavor-mixing for $\sin \theta=0.2$ and $x \sim 1$ (it is shown later that these values are consistent with the $\mu \rightarrow e \gamma$ limit) is

$$
\begin{aligned}
S_{F V}= & N\left(\chi_{2}^{0} \chi_{2}^{0}\right) \times B R\left(\chi_{2}^{0} \rightarrow e e \chi_{1}^{0}, \mu \mu \chi_{1}^{0}\right) \times(0.9)^{4} \times \varepsilon_{C U T} \\
& \times 2 \times 0.24 \times 2 \sin ^{2} \theta \cos ^{2} \theta x \\
\approx & 20 \times \varepsilon_{C U T} .
\end{aligned}
$$

There is an extra factor of 2 since either $\chi_{2}^{0}$ can decay to flavor violating dileptons. Next, the number of $3 e 1 \mu+3 \mu 1 e$ events from leptonic decays of $\tau$ s produced from $\chi_{2}^{0}$ is

$$
\begin{aligned}
B_{F V}= & N\left(\chi_{2}^{0} \chi_{2}^{0}\right) \times 2 \times\left(R_{\tau} \times(0.35)^{2} \times \frac{1}{2}\right) \\
& \times B R\left(\chi_{2}^{0} \rightarrow e e \chi_{1}^{0}, \mu \mu \chi_{1}^{0}\right) \times(0.9)^{4} \times \varepsilon_{4 l} \times \varepsilon_{C U T} \\
\approx & 9 \times \varepsilon_{C U T} .
\end{aligned}
$$

Here, $\varepsilon_{4 l}$ is the acceptance for 4 leptons with 2 of them coming from the decay chain $\chi_{2}^{0} \rightarrow \tau \tau$ relative to that for all 4 leptons coming from $\chi_{2}^{0} \rightarrow \tilde{e} e$ or $\mu \tilde{\mu}$. Since

\footnotetext{
${ }^{10}$ Detection efficiency refers to the probability that the lepton (or $\tau$-jet in a later case) will be detected given that it passes the acceptance cuts.
} 
the leptons from the $\tau$ decay are softer, it is expected that $\varepsilon_{4 l} \lesssim 1{ }^{11}$ To get the number in the last line above, $\varepsilon_{4 l} \approx 1$ has been assumed.

Finally, the above 2 numbers are from the leptonic decays of $2 \chi_{2}^{0} \mathrm{~s}$ from the decays of first two generation squarks only. As mentioned before, stop/sbottom decays to $W, \chi_{3}^{0}$ etc. can give a background to the flavor violating 4-lepton signal (see Eqn.(4.16)). To reject these events, a $b$-jet veto is used. This implies that events with 4 leptons coming from $2 \chi_{2}^{0}$ decays with (at least) one $\chi_{2}^{0}$ coming from a stop/sbottom decay will also be rejected; this is the reason for not including the $\chi_{2}^{0}$ pairs from stop/sbottom decays in the numbers above.

Measuring the background from $\chi_{2}^{0} \rightarrow \tau \tau$ decays is discussed next.

As mentioned earlier, the idea is to measure the number of $(3 e \tau-$ jet $)+$ $(2 e 1 \mu \tau-$ jet $)+\cdots$ events where $\tau$-jet refers to the hadronic decay of $\tau$. At this LHC Point the number of these events (including detection efficiencies) is

$$
\begin{aligned}
N(3 l+\tau-\text { jet })= & N\left(\chi_{2}^{0} \chi_{2}^{0}\right) \times 2 \times\left(R_{\tau} \times 2 \times 0.35 \times \varepsilon_{\tau}\right) \times \varepsilon_{C U T} \times \\
& B R\left(\chi_{2}^{0} \rightarrow e e \chi_{1}^{0}, \mu \mu \chi_{1}^{0}, e \mu \chi_{1}^{0}\right) \times(0.9)^{3} \times \varepsilon_{3 l} .
\end{aligned}
$$

A factcr of 2 is due to either $\tau$ decaying to a jet. Here, $\varepsilon_{\tau}$ includes $B R(\tau \rightarrow$ hadron $)$ and the efficiency for detecting a hadronic decay of $\tau$. The variable $\varepsilon_{3 l}$ is the acceptance for $(3+1 \tau-$ jet $)$ relative to that for 4 leptons all of which come from the decay chain $\chi_{2}^{0} \rightarrow \tilde{e} e, \tilde{\mu} \mu$. It is expected that $1 \gtrsim \varepsilon_{3 l} \gtrsim \varepsilon_{4 l}$ since the lepton from the $\tau$

\footnotetext{
${ }^{11}$ Strictly speaking, the factor $\varepsilon_{4 l}$ should be included in determining $N(4 l)$ and $S_{F V}$ as well. But since the number of events in these samples from $\tau$ decays is very small, it is a good approximation to assume $\varepsilon_{4 l} \approx 1$ in those numbers.
} 
decay is softer than the $\tau$-jet and since the $\tau$ decay products (both lepton and jet) are softer than the leptons from the decay chain $\chi_{2}^{0} \rightarrow \tilde{e} e, \tilde{\mu} \mu$. From Eqns.(4.31) and (4.32) and assuming $B R\left(\chi_{2}^{0} \rightarrow e e \chi_{1}^{0}, \mu \mu \chi_{1}^{0}\right) \approx B R\left(\chi_{2}^{0} \rightarrow e e \chi_{1}^{0}, \mu \mu \chi_{1}^{0}, e \mu \chi_{1}^{0}\right)$, the following relation is obtained

$$
B_{F V} \approx N(3 l+\tau-\mathrm{jet}) \times \frac{0.9 \times \frac{0.35}{2} \times \varepsilon_{4 l}}{2 \times \varepsilon_{3 l} \times \varepsilon_{\tau}}
$$

Note that $R_{\tau}$ cancels in the ratio. Thus, using the $(3 l+\tau-$ jet $)$ detection together with an understanding of the $\tau$ detection efficiency $\left(\varepsilon_{\tau}\right)$, as well as the acceptance for 4 leptons (with 2 of them from $\tau$ decays) versus ( 3 leptons $+\tau$-jet) $\left(\varepsilon_{4 l} / \varepsilon_{3 l}\right)$, the number of (3e $1 \mu+3 \mu 1 e)$ events from $\tau$ decay (Eqn.(4.31)) contained in the full 4-lepton sample can be obtained from the above relation. This is important, as it means that the $\chi_{2}^{0} \rightarrow \tau \tau$ background to the flavor-violating signal can be determined without knowing the relative branching fraction of $\chi_{2}^{0}$ to $h, \tilde{l} l$, or $\tilde{\tau} \tau$.

Assuming that the detection efficiency for the decay $\tau \rightarrow \pi \nu$ (which has a BR of 0.11 ) is 0.9 so that $\varepsilon_{\tau} \approx 0.9 \times 0.11$, and assuming $\varepsilon_{3 l} \approx 1$ gives

$$
N(3 l+\tau-\mathrm{jet}) \approx 11 \times \varepsilon_{C U T} .
$$

Independent of this, it is worth remarking that with enough statistics it might be possible to measure $B R\left(\chi_{2}^{0} \rightarrow h \chi_{1}^{0}\right), B R\left(\chi_{2}^{0} \rightarrow \tilde{e} e, \tilde{\mu} \mu\right)$ and $B R\left(\chi_{2}^{0} \rightarrow \tilde{\tau} \tau\right)$ assuming that these are the dominant decay modes of $\chi_{2}^{0}$. The decay chain $\chi_{2}^{0} \rightarrow$ $h \chi_{1}^{0} \rightarrow b \bar{b} \chi_{1}^{0}$ (where $\chi_{2}^{0}$ is from cascade decays of squarks as usual) gives $b \bar{b}$ events with high $p_{T}$ jets and $\not_{T}$. Comparing these to the number of dilepton events from 
$\chi_{2}^{0}$ decays gives

$$
\frac{N(b \bar{b})}{N(2 l)} \propto \frac{B R\left(\chi_{2}^{0} \rightarrow h \chi_{1}^{0}\right)}{B R\left(\chi_{2}^{0} \rightarrow \tilde{e} e \chi_{1}^{0}, \tilde{\mu} \mu \chi_{1}^{0}\right)}
$$

Similarly, the number of $3 l+1 \tau$-jet events compared to 4 -lepton events is

$$
\frac{N(3 l+\tau-\mathrm{jet})}{N(4 l)} \propto \frac{R_{\tau}}{B R\left(\chi_{2}^{0} \rightarrow \tilde{e} e \chi_{1}^{0}, \tilde{\mu} \mu \chi_{1}^{0}\right)}
$$

All the events in the above two equations have in addition high $p_{T}$ jets and $p_{T}$ to make sure that these are from cascade decays of squarks. From these two measurements and the assumption that $\sum B R s=1$ the above--mentioned branching ratios can be obtained. This could provide complementary information to the flavor violating signal disc-3sed here.

Returning to the main subject of this section, an observation of an excess of the 'flavor violating' 4-leptons events over those from $\tau$ decay (Eqn.(4.31)) would be a strong evidence for lepton flavor violation. But, before concluding that SUSY lepton flavor violation has been detected, the background to the flavor violating 4 lepton events from stop/sbottom production (see Eqn.(4.37) below) must be removed, and also the $\tau$-jet detection efficiency $\varepsilon_{\tau}$ must be known. These two issues are discussed next.

The $\tau$ hadronic decays from $Z \rightarrow \tau \tau$ at the LHC were simulated for the ATLAS detector in $[60]^{12}$. This study shows that a detection efficiency $\epsilon_{\tau}$ for a hadronic $\tau$ decay (including the multi-prong decays, i.e, a total $\tau$ decay $\mathrm{BR}$ of 0.65 ) of $\approx 40 \times 0.65 \%$ with a rejection factor of 15 for non- $\tau$ jets can be achieved. This is

\footnotetext{
${ }^{12}$ There is also a study of detecting $\tau$-jets from heavy SUSY Higgs decay for the CMS detector $[61]$.
} 
possible since $\tau$-jets have lower particle multiplicity, narrower profile and smaller invariant mass than the QCD jets [60]. A similar detection efficiency (or even better detection efficiency and rejection of non- $\tau$ jets if the strategy is optimized for this case) for $\tau$-jets from sparticle decays could be expected.

The important point about this though is that it suffices to know a lower limit on the $\tau$-jet detection efficiency to get an upper limit on the number of $(3 e 1 \mu)+(3 \mu 1 e)$ events from tau decays using the $(3 e \tau-$ jet $)$ events (see Eqn.(4.33)). Similarly, since $\varepsilon_{4 l} \lesssim \varepsilon_{3 l}$, an upper limit on $B_{F V}$ can be obtained even though these $\varepsilon$ 's may not be known precisely. Also, if the $\tau$-jet detection (and QCD jet rejection) is good, there will be large number of events with 2 leptons and $2 \tau$-jets from $2 \chi_{2}^{0}$ decays. These can be used in addition to the 3 lepton $1 \tau$-jet events to estimate the background to flavor violating 4 lepton events from $\tilde{\tau} / \tau$ decays.

To reduce the stop and sbottom backgrounds a $b$-jet veto can be used. Before using this veto, the number of expected $3 e 1 \mu+3 \mu 1 e$ events from decays of $\tilde{t}$ or $\tilde{b}$ to $W, \chi_{3}^{0}, \chi_{1}^{ \pm}$etc. (in the absence of any flavor mixing) can be estimated using the production cross-sections and branching fractions from Tables 4.2 and 4.3. The result is, including lepton detection efficiencies:

$$
N(\tilde{t} \text { or } \tilde{b}) \approx 50 \times \varepsilon_{C U T}
$$

Each of these events has at least $2 b$ quarks. So with a $b$-detection efficiency of $60 \%$ (and rejection factor of 200 against non- $b$ jets at low luminosity [62]), the 
number of $3 e 1 \mu+\cdots$ events from stop/sbottom decays after the $b$-jet veto goes down to 8 . This can be further reduced by using a $b$-tagging efficiency of $90 \%$ with a mistagging rate of $25 \%$ (i.e., rejection factor of 4 against non- $b$ jets) at low luminosity $[56,62]$; this will reduce the signal by a bit. This strategy can be optimized depending on the luminosity [62].

Lastly, to get actual number of events, the cuts used to select these events must also be taken into account. The effect of these cuts on the signal and background rates is buried in the fudge factor $\varepsilon_{C U T}$. For example, $p_{T} \gtrsim 10 \mathrm{GeV}$ and $|\eta| \lesssim 2.5$ is required to be able to detect $e$ or $\mu$. Also, to reduce any remaining small SM background, i.e., to make sure that these are SUSY events, various cuts on $\not p_{T}, p_{T}$ of jets, a variable $M_{\text {eff }}[55,56]$ related to $p_{T}, p_{T}$ of jets, can be imposed. Analysis of the events simulated in [56] showed that there were $\sim 40$ events with 4 leptons with no $b$-jets that pass all the cuts mentioned above compared to the estimate of $\sim 149$ from cross-sections and BRs, Eqn.(4.29): there is an acceptance factor of $\varepsilon_{C U T} \sim 1 / 4$ from the various kinematic cuts. We have also checked that almost all of these (simulated) events have $2 \chi_{2}^{0} \mathrm{~s}$ as expected. ${ }^{13}$ There are very few events in this sample (from the simulation) with heavier neutralinos/chargino in agreement with the expectation from the very small BRs of the first two generation squarks to these sparticles at this point in the SUSY parameter space [56] (see Table 4.3). The number of events (from the simulation) with at least $1 b$ quark and 4 leptons

\footnotetext{
${ }^{13}$ The information about whether an event in the simulation has $\chi_{2}^{0} \mathrm{~s}, \tilde{t} \mathrm{~s}, \chi_{3}^{0} \mathrm{~s}$ etc. is from the event generator.
} 
is also in rough agreement (up to the acceptance factor) with the number of 4 lepton events with at least 1 stop/sbottom expected from the cross-section and branching fraction estimates. ${ }^{14}$

Including an acceptance factor of $\varepsilon_{C U T} \sim 1 / 4$ for both background and signal, a $b$-jet detection efficiency of $60 \%$ (which was not included in Eqn.(4.37)) and detection efficiency of $90 \%$ for the decay $\tau \rightarrow \pi \nu$, and a $66 \%$ 4-lepton detection efficiency (the $\tau$ and lepton detection efficiencies were included in the previous estimates of $S_{F V}$ etc.), a summary of the expected number of events at low luminosity is :

$$
\begin{aligned}
N(4 l) & \approx 37 \times \frac{L}{10 \mathrm{fb}^{-1}} \\
S_{F V} & \approx 5 \times \frac{\sin ^{2} 2 \theta}{0.15} x \times \frac{L}{10 \mathrm{fb}^{-1}} \\
B_{F V} & \approx 2 \times \frac{L}{10 \mathrm{fb}^{-1}} \\
N(3 l+\tau-\text { jet }) & \approx 3 \times \frac{L}{10 \mathrm{fb}^{-1}} \\
N(\tilde{t} \text { or } \tilde{b}) & \approx 2 \times \frac{L}{10 \mathrm{fb}^{-1}} .
\end{aligned}
$$

While these numbers may be a little small for one detector and one year of running at low luminosity $\left(L=10 \mathrm{fb}^{-1}\right)$, there is cause for optimism. More integrated luminosity $L$ from $>1$ year of running and/or 2 detectors can significantly increase the statistics. Further, a larger $B R\left(\chi_{2}^{0} \rightarrow \tilde{l l}\right)$ would give more statistics. This could occur at a point in the SUSY parameter space with a heavier Higgs boson, and

\footnotetext{
${ }^{14}$ We have also checked that these simulated events do have at least 1 stop/sbottom. There are very few events in this sample with $n o$ stops/sbottoms but with $b$-jets from initial state gluon radiation.
} 
thus a lower $B R\left(\chi_{2}^{0} \rightarrow h \chi_{1}^{0}\right)$.

To illustrate the discovery or exclusion significance of these results, an integrated luminosity of $L=100 \mathrm{fb}^{-1}$ is considered. This could occur for 5 years of running at low luminosity for two detectors ${ }^{15}$. For this integrated luminosity there are 22 4-lepton flavor violating events from the $\tilde{\tau} / \tau$ background, and 30 3-lepton $\tau$-jet events. There will also be 125 4-lepton flavor violating events before the $b$-jet veto from the $\tilde{t} / \tilde{b}$ background. Next, the $b$-tagging efficiency is optimized so that the $\tilde{t} / \tilde{b}$ background is (less than or) equal to the $1 \sigma$ statistical error in $\tilde{\tau} / \tau$ background while at the same time the reduction of the signal due to mistagging is small. This is achieved with a $b$-tagging efficiency of $80 \%$, rather than the $60 \%$ of before. At this higher tagging efficiency there is a mistagging rate of 1 in 50 , so there is very little reduction of the signal. With an $80 \% b$-tagging efficiency, $5 \tilde{t} / \tilde{b}$ background events remain since each event has at least $2 b$-jets. Then the background is dominated by the $\tilde{\tau} / \tau$ decays. A $5 \sigma(2 \sigma)$ discovery (exclusion) requires that $S / \sqrt{B}>5(S / \sqrt{B}>2)$, and this requires $>23(>9)$ signal events. So a $5 \sigma$ discovery is obtained for

$$
\sqrt{x} \sin 2 \theta_{R}>0.26(5 \sigma \text { discovery }) \text { or } \sin \theta_{R}>0.13 \text { for } x \sim 1 .
$$

\footnotetext{
${ }^{15}$ One year of running at high luminosity is also possible. In this case however, the $b$-jet mistagging rate increases to 1 in 6 for a $b$-tagging efficiency of $80 \%$ [62]. Since most of the signal events occur from $\tilde{q} \tilde{g}$ production and so contain at least three hard jets, approximately $40 \%$ of the signal could be rejected. In this case the discovery (and exclusion) limits on $\sin \theta_{R}$ increase by about $25 \%$. In addition, the tau-jet detection efficiency at high luminosity is not known since a low luminosity was used in the ATLAS study.
} 
If no signal is observed then the $2 \sigma$ exclusion limit is

$\sqrt{x} \sin 2 \theta_{R}>0.16(2 \sigma$ exclusion $)$ or $\sin \theta_{R}>0.08$ for $x \sim 1$.

To end this section, these values of $\sin 2 \theta_{R}$ and $\Delta m / m$ that may be probed by the LHC are compared to the constraints on these parameters obtained from $\mu \rightarrow e \gamma$. The LHC signal is proportional to $\sin ^{2} 2 \theta_{R} x$, with $x \sim 1$ if $\Delta m \gtrsim \Gamma$ and $x \ll 1$ if $\Delta m \ll \Gamma$. The decay $\mu \rightarrow e \gamma$ places an upper limit on $\sin 2 \theta_{R} \Delta m / m$ (Eqn.(4.4)) so that there is competition between the two probes of flavor violation. Thus, in order for the signal at the LHC to be significant in the region of the $\left(\sin 2 \theta_{R}, \Delta m / m\right)$ plane beyond the reach of the $\mu \rightarrow e \gamma$ limit, there should be a range of $\Delta m / m$ where $\Delta m \gtrsim \Gamma$ so that $x \sim 1$ and $\Delta m / m$ is small enough (for a given value of $\left.\sin 2 \theta_{R}\right)$ so that $\mu \rightarrow e \gamma$ is suppressed. It will be seen that for $\Delta m / m \sim \Gamma / m$ (so that $x \sim 1$ ), at this LHC Point, $\sin 2 \theta_{R}$ is unconstrained by the $\mu \rightarrow e \gamma$ limit, affording the LHC the opportunity to either detect a signal or extend the limit.

At this Point $\chi_{1}^{0} \approx \tilde{B}^{0}$. A computation of the one-loop $\tilde{B}^{0}$ contribution gives

$$
\sin 2 \theta_{R} \frac{\Delta m_{R}^{2}}{\tilde{m}_{R}^{2}}\left(\frac{100 \mathrm{GeV} \tilde{m}_{R}}{M_{\chi_{1}^{0}}^{2}}\right)^{2} 20 F\left(\alpha_{L}, \alpha_{R}, t\right)<0.013 \times \sqrt{\frac{B R(\mu \rightarrow e \gamma)}{4.9 \times 10^{-11}}} .
$$

Here $\alpha_{H}=\tilde{m}_{H}^{2} / M_{\chi_{1}^{0}}^{2}(H=L, R), t=(A+\mu \tan \beta) / \tilde{m}_{R}$,

$$
F\left(\alpha_{L}, \alpha_{R}, t\right)=H\left(\alpha_{R}\right)+t \alpha_{R}^{1 / 2} \frac{\partial K}{\partial \alpha_{R}}\left(\alpha_{L}, \alpha_{R}\right)
$$

with

$$
K(x, y)=\frac{g(x)-g(y)}{x-y}, g(x)=\frac{1+2 x \log x-x^{2}}{2(x-1)^{3}}
$$


and

$$
H(x)=\frac{-x^{3}+9 x^{2}+9 x-17-(6+18 x) \log x}{6(x-1)^{5}}
$$

Two useful facts are $H(1)=\frac{\partial}{\partial y} K(1,1)=-1 / 20$; hence the factor of 20 on the left side of Eqn.(4.41). At this LHC Point, $m_{\tilde{l}_{R}} \sim 160 \mathrm{GeV}, m_{\chi_{1}^{0}} \sim 120 \mathrm{GeV}$, and $m_{\tilde{l}_{L}} \sim 240 \mathrm{GeV}$. Inputing these masses into the above formula simplifies it to:

$$
\frac{\sin 2 \theta_{R}}{0.39} \times \frac{\Delta m_{R}}{\tilde{m}_{R}} \times(1+0.48 t)<0.03 \times \sqrt{\frac{B R(\mu \rightarrow e \gamma)}{4.9 \times 10^{-11}}} .
$$

At this Point $t \approx 5-10$. However, a larger variation in $t$ is allowed without affecting the flavor violating signal, since both $A$ and $\operatorname{sgn}(\mu)$ do not qualitatively affect the 4 -lepton event rate ${ }^{16}$. In any case, the values $\sin 2 \theta_{R} \approx 0.39$ and $\Delta m_{R} \sim \Gamma$ (so that $x \sim 1$ ) with a typical value of $\Gamma \sim \alpha_{e m} m \sim 10^{-2} \times \tilde{m}_{R}$ are consistent with $\mu \rightarrow e \gamma-$ recall that $\sin 2 \theta_{R} \approx 0.39$ and $x \sim 1$ was assumed to obtain the estimate of $S_{F V}$ in Eqn.(4.30). In fact, at this LHC Point $\Gamma \sim 125$ $\mathrm{MeV}[56]$, so that $\Gamma / m \sim 8 \times 10^{-4}$ which is smaller than $\alpha_{e m}$. So for $\Delta m / m \gtrsim$ $2 \Gamma / m \approx 1.6 \times 10^{-3}$, it follows that $x \sim 1$. From Eqn.(4.45) and for maximal mixing $\left(\sin 2 \theta_{R}=1\right), \Delta m / m<0.39 \times 0.03 /(1+0.48 t) \approx 4 \times 10^{-3}($ for $t \approx 5)$. Thus for $1.6 \times 10^{-3} \lesssim \Delta m / m \lesssim 4 \times 10^{-3}$ and $\sin 2 \theta_{R}=1, \mu \rightarrow e \gamma$ is satisfied and $x \sim 1$. So at this Point even for maximal mixing there is a large range of $\Delta m / m$ for which $x \sim 1$ and $\mu \rightarrow e \gamma$ is safe. Of course, smaller mixing could be probed by the LHC, in which case the upper bound on $\Delta m / m$ allowed by $\mu \rightarrow e \gamma$ is larger. In this case for a given $\sin \theta_{R}$ there is a larger range of $\Delta m / m$ for which $x \sim 1$ (so that there is no suppression of the LHC signal) and $\mu \rightarrow e \gamma$ is safe.

\footnotetext{
${ }^{16} \mathrm{It}$ is important to maintain the relation $m_{\chi_{2}^{0}}>m_{\tilde{e}_{R}}$ though.
} 


\subsection{Summary}

The point of this Chapter is to demonstrate that it is possible to detect SUSY lepton flavor violation at the LHC using events with 4 leptons from the cascade decays of squarks provided the following conditions are satisfied:

0 . Either $R$-parity is conserved or $\chi_{1}^{0}(L S P)$ decays outside the detector,

1. $\chi_{2}^{0}$ pair production in cascade decays of squarks is large and $\chi_{2}^{0}$ has a large decay branching fraction to $\tilde{l}^{*} l$ (to get enough statistics),

2. Hadronic decays of $\tau \mathrm{s}$ can be detected with a known efficiency so that the background from the $\chi_{2}^{0} \rightarrow \tau \tau$ decay can be estimated,

3. The $b$-jet detection efficiency is good so that the background from events with stop/sbottom can be rejected,

4. The stop/sbottom production rate, either direct or in gluino decays, is not too much larger than the production of first two generation squarks,

5. The first two generation squarks decay largely to $\chi_{2}^{0}, \chi_{1}^{0}$ and $\chi_{1}^{ \pm}$, so that the background to flavor violating 4 lepton events from decays of heavier neutralinos/chargino to $W \mathrm{~s}$, lighter chargino, heavier sleptons etc. is small. This condition can be realized in the so-called gaugino-like region,

6. The mass splitting is $\Delta m \sim \Gamma$ or larger, so there is no suppression of the signal due to the quantum interference effect.

The arguments presented here are clearly semi-quantitative, and further study requiring a detailed simulation of these processes is required. 


\section{Chapter 5}

\section{Finetuning in Low-Energy Gauge Mediation}

The fine tuning in models of low energy gauge mediated supersymmetry breaking required to obtain the correct $Z$ mass is quantified. To alleviate the fine tuning problem, a model with split $(5+\overline{5})$ messenger fields is presented. This model has additional triplets in the low energy theory which get a mass of $O(500) \mathrm{GeV}$ from a coupling to a singlet. The improvement in fine tuning is quantified and the spectrum in this model is discussed. The same model with the above singlet coupled to the Higgs doublets to generate the $\mu$ term is also discussed. A Grand Unified version of the model is constructed and a known doublet-triplet splitting mechanism is used to split the messenger $(5+\overline{5})$ 's. A complete model is presented and some phenomenological constraints are discussed.

\subsection{Introduction}

One of the outstanding problems of particle physics is the origin of electroweak symmetry breaking (EWSB). In the Standard Model (SM), this is achieved by one Higgs doublet which acquires a vacuum expectation value (vev) due to a negative mass squared which is put in by hand. The SM has the well known

gauge hierarchy problem [4]. It is known that supersymmetry (SUSY) stabilizes 
the hierarchy between the weak scale and some other high scale without any fine tuning if the masses of the superpartners are less than few $\mathrm{TeV}[63,64]$. The Minimal Supersymmetric Standard Model (MSSM) is considered as a low energy effective theory in which the soft SUSY breaking terms at the correct scale are put in by hand. This raises the question : what is the origin of these soft mass terms, i.e., how is SUSY broken? If SUSY is broken spontaneously at tree level in the MSSM, then there is a colored scalar lighter than the up or down quarks [65]. So, the superpartners have to acquire mass through either radiative corrections or non-renormalizable operators. Thus there is a "hidden" sector where SUSY is broken spontaneously at tree-level and then communicated to the MSSM by some "messengers".

There are two problems here: how is SUSY broken in the hidden sector at the right scale and what are the messengers? There are models in which a dynamical superpotential is generated by non-perturbative effects which breaks SUSY [66]. The SUSY breaking scale is related to the Planck scale by dimensional transmutation. Two possibilities have been discussed in the literature for the messengers. One is gravity which couples to both the sectors [67]. In a supergravity theory, there are non-renormalizable couplings between the two sectors which generate soft SUSY breaking operators in the MSSM once SUSY is broken in the "hidden" sector. In the absence of a flavor symmetry, this theory has to be fine tuned to give almost degenerate squarks and sleptons of the first two generations which is required by Flavor Changing Neutral Current (FCNC) phenomenology 
$[65,68]$. The other messengers are the SM gauge interactions [11]. In these models, the scalars of the first two generations are naturally degenerate since they have the same gauge quantum numbers. This is an attractive feature of these models, since the FCNC constraints are naturally avoided and no fine tuning between the masses of the first two generation scalars is required. If this lack of fine tuning is a compelling argument in favor of these models, then it is important to investigate whether other sectors of these models are fine tuned. In fact, it will be argued (and this is also discussed in $[69,70,71]$ ) that the minimal model (to be defined in section 5.2) of low-energy gauge-mediated SUSY breaking requires a minimum $7 \%$ fine tuning to generate a correct vacuum ( $Z$ mass) if no superpartners are discovered at LEP2. Further, if a gauge-singlet and extra vector-like quintets are introduced to generate the " $\mu$ " and " $B \mu$ " terms, then the minimal model of low energy gauge-mediated SUSY breaking requires a few percent fine tuning to correctly break the electroweak symmetry. These fine tunings makes it difficult to understand, within the context of these models, how SUSY is to offer some understanding of the origin of electroweak symmetry breaking and the scale of the $Z$ and $W$ gauge boson masses.

This chapter is organized as follows. Section 5.2 reviews both the "messenger sector" in low energy gauge-mediated SUSY breaking models that communicates SUSY breaking to the Standard Model and the pattern of the sfermion and gaugino masses that follow. Section 5.3 quantifies the fine tuning in the minimal model using the Barbieri-Giudice criterion [63]. Section 5.4 describes a toy model with 
split $(5+\overline{5})$ messenger representations that improves the fine tuning. To maintain gauge coupling unification, additional triplets are added to the low energy theory. They acquire a mass of $O(500) \mathrm{GeV}$ by a coupling to a singlet. The fine tuning in this model is improved to $\sim 40 \%$. The sparticle phenomenology of these models is also discussed. Section 5.5 discusses a version of the toy model where the above mentioned singlet generates the $\mu$ and $\mu_{3}^{2}$ terms. This is identical to the Nextto-Minimal Supersymmetric Standard Model (NMSSM) [72] with a particular pattern for the soft SUSY breaking operators that follows from gauge-mediated SUSY breaking and this particular solution to the fine tuning problem. Then this model is shown to be tuned to $\sim 20 \%$, even if LEP does not discover SUSY/light Higgs. It is also shown that the NMSSM with one complete messenger $(5+\overline{5})$ is fine tuned to $\sim 2 \%$. Section 5.6 discusses how it is possible to make this toy model compatible with a Grand Unified Theory (GUT) [13] based upon the gauge group $S U(5) \times S U(5)$. The doublet-triplet splitting mechanism of Barbieri, Dvali and Strumia [73] is used to split both the messenger representations and the Higgs multiplets. Section 5.7 presents a model in which all operators consistent with symmetries are present and for which the low energy theory is thə model of section 5.5. In this model $R$-parity $\left(R_{p}\right)$ is the unbroken subgroup of a $Z_{4}$ global discrete symmetry that is required to solve the doublet-triplet splitting problem. This model has some metastable particles which might cause a cosmological problem. Appendix A gives the expressions for the Barbieri-Giudice parameters (for the fine tuning) for the MSSM and the NMSSM. 


\subsection{Messenger Sector}

In the models of low energy gauge-mediated SUSY breaking $[69,74]$ (henceforth called LEGM models), SUSY breaking occurs dynamically in a "hidden" sector of the theory at a scale $\Lambda_{d y n}$ that is generated through dimensional transmutation. SUSY breaking is communicated to the Standard Model fields in two stages. First, a non-anomalous $U(1)$ global symmetry of the hidden sector is weakly gauged. This $U(1)_{X}$ gauge interaction communicates SUSY breaking from the original SUSY breaking sector to a messenger sector at a scale $\Lambda_{\text {mess }} \sim \alpha_{X} \Lambda_{d y n} /(4 \pi)$ as follows. The particle content in the messenger sector consists of fields $\phi_{+}, \phi_{-}$charged under this $U(1)_{X}$, a gauge singlet field $S$, and vector-like fields that carry Standard Model quantum numbers (henceforth called messenger quarks and leptons). In the minimal LEGM model, there is one set of vector-like fields, $\bar{q}, l$, and $q, \bar{l}$ that together form a $(\overline{5}+5)$ of $S U(5)$. This is a sufficient condition to maintain unification of the SM gauge couplings. The superpotential in the minimal model is

$$
W_{m e s s}=\lambda_{\phi} \phi_{+} \phi_{-} S+\frac{1}{3} \lambda_{S} S^{3}+\lambda_{q} S q \bar{q}+\lambda_{l} S l \bar{l} .
$$

The scalar potential is

$$
V=\sum_{i}\left|F_{i}\right|^{2}+m_{+}^{2}\left|\phi_{+}\right|^{2}+m_{-}^{2}\left|\phi_{-}\right|^{2}
$$

In the models of $[69,74]$, the $\phi_{+}, \phi_{-}$fields communicate (at two-loops) with the hidden sector fields through the $U(1)$ gauge interactions. Then, SUSY breaking in 
the original sector generates a negative value $\sim-\left(\alpha_{X} \Lambda_{d y n}\right)^{2} /(4 \pi)^{2}$ for the mass parameters $m_{+}^{2}, m_{-}^{2}$ of the $\phi_{+}$and $\phi_{-}$fields. This drives vevs of $O\left(\Lambda_{\text {mess }}\right)$ for the scalar components of both $\phi_{+}$and $\phi_{-}$, and also for the scalar and $F$-component of $S$ if the couplings $\lambda_{S}, g_{X}$ and $\lambda_{\phi}$ satisfy the inequalities derived in $[70,75]{ }^{1}$ Generating a vev for both the scalar and $F$-component of $S$ is crucial, since this generates a non-supersymmetric spectrum for the vector-like fields $q$ and $l$. The spectrum of each vector-like messenger field consists of two complex scalars with masses $M^{2} \pm B$ and two Weyl fermions with mass $M$ where $M=\lambda S, B=\lambda F_{S}$ and $\lambda$ is the coupling of the vector-like fields to $S$. Since the breaking of the SM at this stage is undesired, $M^{2}-B \geq 0$. In the second stage, the messenger fields are integrated out. As these messenger fields have SM gauge interactions, SM gauginos acquire masses at one loop and the sfermions and Higgs acquire soft scalar masses at two-loops [11]. The gaugino masses at the scale at which the messenger fields are integrated out, $\Lambda_{\text {mess }} \approx M$ are [74]

$$
M_{G}=\frac{\alpha_{G}\left(\Lambda_{m e s s}\right)}{4 \pi} \Lambda_{S U S Y} \sum_{m} N_{R}^{G}(m) f_{1}\left(\frac{F_{S}}{\lambda_{m} S^{2}}\right)
$$

The sum in Eqn.(5.3) is over messenger fields $(m)$ with normalization $\operatorname{Tr}\left(T^{a} T^{b}\right)=N_{R}^{G}(m) \delta^{a b}$ where the $T^{\prime}$ 's are the generators of the gauge group $G$ in the representation $R, f_{1}(x)=1+O(x)$, and $\Lambda_{S U S Y} \equiv B / M=F_{S} / S=x \Lambda_{\text {mess }}$ with $x=B / M^{2} .{ }^{2}$ Henceforth, the approximation $\Lambda_{S U S Y} \approx \Lambda_{\text {mess }}$ is used. The

\footnotetext{
${ }^{1}$ This point in field space is a local minimum. There is a deeper minimum where SM is broken $[70,75]$. This problem is avoided by adding another singlet to the messenger sector [70]. This does not change the conclusions found here about the fine tuning.

${ }^{2}$ If all the dimensionless couplings in the superpotential are of $O(1)$, then $x$ cannot be much
} 
exact one-loop calculation [76] of the gaugino mass shows that $f_{1}(x) \leq 1.3$ for $x \leq 1$. The soft scalar masses at $\Lambda_{\text {mess }}$ are [74]

$$
m_{i}^{2}=2 \Lambda_{S U S Y}^{2} \sum_{m, G} N_{R}^{G}(m) C_{R}^{G}\left(s_{i}\right)\left(\frac{\alpha_{G}\left(\Lambda_{m e s s}\right)}{4 \pi}\right)^{2} f_{2}\left(\frac{F_{S}}{\lambda_{m} S^{2}}\right)
$$

where $C_{R}^{G}\left(s_{i}\right)$ is the Casimir of the representation of the scalar $i$ in the gauge group $G$ and $f_{2}(x)=1+O(x)$. The exact two-loop calculation [76] which determines $f_{2}$ shows that for $x \leq 0.8(0.9), f_{2}$ differs from one by less than $1 \%(5 \%)$. Henceforth $f_{1}(x)=1$ and $f_{2}(x)=1$ are used. In the minimal LEGM model

$$
\begin{gathered}
M_{G}\left(\Lambda_{\text {mess }}\right)=\frac{\alpha_{G}\left(\Lambda_{\text {mess }}\right)}{4 \pi} \Lambda_{\text {mess }} \\
m^{2}\left(\Lambda_{\text {mess }}\right)= \\
\left(C_{3}\left(\frac{\alpha_{3}\left(\Lambda_{\text {mess }}\right)}{4 \pi}\right)^{2}+C_{2}\left(\frac{\alpha_{2}\left(\Lambda_{\text {mess }}\right)}{4 \pi}\right)^{2}+\frac{3}{5}\left(\frac{\alpha_{1}\left(\Lambda_{\text {mess }}\right) Y}{4 \pi}\right)^{2}\right) \\
(5.6)
\end{gathered}
$$

where $Q=T_{3 L}+Y$ and $\alpha_{1}$ is the $S U(5)$ normalized hypercharge coupling. Further, $C_{3}=4 / 3$ and $C_{2}=3 / 4$ for colored triplets and electroweak doublets respectively.

The spectrum in the models is determined by only a few unknown parameters. As Eqns.(5.3) and (5.4) indicate, the SUSY breaking mass parameters for the Higgs, sfermions and gauginos are

$$
m_{\tilde{q}}, m_{\tilde{g}}: m_{\tilde{L}}, m_{H_{i}}, m_{\tilde{W}}: m_{\tilde{e}_{R}}, m_{\tilde{B}} \sim \alpha_{3}: \alpha_{2}: \alpha_{1}
$$

The scale of $\Lambda_{\text {mess }}$ is chosen to be $\sim 100 \mathrm{TeV}$ so that the lightest of these particles escapes detection. It follows that the intrinsic scale of supersymmetry breaking, smaller than 1. 
$\Lambda_{d y n}$, is $\sim 10000 \mathrm{TeV}$. The goldstino decay of the lightest Standard Model superpartner then occurs outside the detector [77]. The phenomenology of the minimal LEGM model is discussed in detail in [77].

\subsection{Fine Tuning in the Minimal LEGM}

A desirable feature of gauge mediated SUSY breaking is the natural suppression of FCNC processes since the scalars with the same gauge quantum numbers are degenerate [11]. But, the minimal LEGM model introduces a fine tuning in the Higgs sector unless the messenger scale is low. This has been previously discussed in $[69,70]$ and quantified more recently in [71]. This discussion is outlined in order to introduce some notation.

The superpotential for the MSSM is

$$
W=\mu H_{u} H_{d}+W_{Y u k a w a} .
$$

The scalar potential is

$$
V=\mu_{1}^{2}\left|H_{u}\right|^{2}+\mu_{2}^{2}\left|H_{d}\right|^{2}-\left(\mu_{3}^{2} H_{u} H_{d}+\text { h.c. }\right)+\mathrm{D} \text {-terms }+V_{1-l o o p}
$$

where $V_{1-\text { loop }}$ is the one-loop effective potential. The vev of $H_{u}\left(H_{d}\right)$, denoted by $v_{u}\left(v_{d}\right)$, is responsible for giving mass to the up (down)-type quarks, $\mu_{1}^{2}=m_{H_{d}}^{2}+\mu^{2}$, $\mu_{2}^{2}=m_{H_{u}}^{2}+\mu^{2}$ and $\mu_{3}^{2},{ }^{3} m_{H_{u}}^{2}, m_{H_{d}}^{2}$ are the SUSY breaking mass terms for the

\footnotetext{
${ }^{3} \mu_{3}^{2}$ is often written as $B \mu$.
} 
Higgs fields. ${ }^{4}$ Extremizing this potential determines, with $\tan \beta \equiv v_{u} / v_{d}$,

$$
\begin{gathered}
\frac{1}{2} m_{Z}{ }^{2}=\frac{\tilde{\mu}_{1}^{2}-\tilde{\mu}_{2}^{2} \tan ^{2} \beta}{\tan ^{2} \beta-1}, \\
\sin 2 \beta=2 \frac{\mu_{3}^{2}}{\tilde{\mu}_{1}^{2}+\tilde{\mu}_{2}^{2}},
\end{gathered}
$$

where $\tilde{\mu}_{i}^{2}=\mu_{i}^{2}+2 \partial V_{1-\text { loop }} / \partial v_{i}^{2}$. For large $\tan \beta, m_{Z}^{2} / 2 \approx-\left(m_{H_{u}}^{2}+\mu^{2}\right)$. This indicates that if $\left|m_{H_{u}}^{2}\right|$ is large relative to $m_{Z}^{2}$, the $\mu^{2}$ term must cancel this large number to reproduce the correct value for $m_{Z}^{2}$. This introduces a fine tuning in the Higgs potential, that is naively of the order $m_{Z}^{2} /\left(2\left|m_{H_{u}}^{2}\right|\right)$. It is demonstrated below that this occurs in the minimal LEGM model.

In the minimal LEGM tiodel, a specification of the messenger particle content and the messenger scale $\Lambda_{\text {mess }}$ fixes the sfermion and gaugino spectrum at that scale. For example, the soft scalar masses for the Higgs fields are $\approx \alpha_{2}\left(\Lambda_{\text {mess }}\right) \Lambda_{\text {mess }} /(4 \pi)$. Renormalization Group (RG) evolution from $\Lambda_{\text {mess }}$ to the electroweak scale reduces $m_{H_{u}}^{2}$ due to the large top quark Yukawa coupling, $\lambda_{t}$, and the squark soft masses. The one loop Renormalization Group Equation (RGE) for $m_{H_{u}}^{2}$ is (neglecting gaugino and the trilinear scalar term $\left(H_{u} \tilde{Q} \tilde{u}^{c}\right)$ contributions )

$$
\frac{d m_{H_{u}}^{2}(t)}{d t} \approx \frac{3 \lambda_{t}^{2}}{8 \pi^{2}}\left(m_{H_{u}}^{2}(t)+m_{\tilde{u}^{c}}^{2}(t)+m_{\tilde{Q}}^{2}(t)\right)
$$

which gives

$$
m_{H_{u}}^{2}\left(t \approx \ln \left(\frac{m_{\tilde{t}}}{\Lambda_{\text {mess }}}\right)\right) \approx m_{H_{u}}^{2}(0)-\frac{3 \lambda_{t}^{2}}{8 \pi^{2}}\left(m_{H_{u}}^{2}(0)+m_{\tilde{u}^{c}}^{2}(0)+m_{\tilde{Q}^{(}}^{2}(0)\right) \ln \left(\frac{\Lambda_{\text {mess }}}{m_{\tilde{t}}}\right)
$$

\footnotetext{
${ }^{4}$ The scale dependence of the parameters appearing in the potential is implicit.
} 
On the right-hand side of Eqn.(5.13) the RG scaling of $m_{\tilde{Q}}^{2}$ and $m_{\tilde{u}^{c}}^{2}$ has been neglected. Since the logarithm $|t| \approx \ln \left(\Lambda_{\text {mess }} / m_{\tilde{t}}\right)$ is small, it is naively expected that $m_{H_{u}}^{2}$ will not be driven negative enough and will not trigger electroweak symmetry breaking. But in this case the squarks are heavy. For example, the squarks are $\approx 500 \mathrm{GeV}(1 \mathrm{TeV})$ for a messenger scale $\Lambda_{\text {mess }}=50 \mathrm{TeV}(100 \mathrm{TeV}$. Thus the radiative corrections from virtual top squarks are large. A numerical solution of the one-loop RGE (including gaugino and the trilinear scalar term $\left(H_{u} \tilde{Q} \tilde{u}^{c}\right)$ contributions) determines $-m_{H_{u}}^{2}=(275 \mathrm{GeV})^{2}\left((550 \mathrm{GeV})^{2}\right)$ for $\Lambda_{m e s s}=50 \mathrm{TeV}(100$ $\mathrm{TeV}$ ) and setting $\lambda_{t}=1$. Therefore, $m_{Z}^{2} /\left(2\left|m_{H_{u}}^{2}\right|\right) \sim 0.06(0.01)$, an indication of the fine tuning required.

To reduce the fine tuning in the Higgs sector, it is necessary to reduce $\left|m_{H_{u}}^{2}\right|$; ideally so that $m_{H_{u}}^{2} \approx-0.5 m_{Z}^{2}$. The large value of $\left|m_{H_{u}}^{2}\right|$ at the weak scale is a consequence of the large hierarchy in the soft scalar masses at the messenger scale: $m_{\tilde{e}_{R}}^{2}<m_{H_{u}}^{2} \ll m_{\tilde{Q}, \bar{u}^{c}}^{2}$. Models of sections 5.4,5.5, and 5.7 attempt to reduce the ratio $m_{\tilde{Q}}^{2} / m_{H_{u}}^{2}$ at the messenger scale and hence improve the fine tuning in the Higgs sector.

The fine tuning may be quantified by applying one of the criteria of $[63,64]$. The value $O^{*}$ of a physical observable $O$ will depend on the fundamental parameters $\left(\lambda_{i}\right)$ of the theory. The fundamental parameters of the theory are to be distinguished from the free parameters of the theory which parameterize the solutions to $O\left(\lambda_{i}\right)=O^{*}$. If the value $O^{*}$ is unusually sensitive to the underlying parameters $\left(\lambda_{i}\right)$ of the theory then a small change in $\lambda_{i}$ produces a large change 
in the value of $O$. The Barbieri-Giudice function

$$
c\left(O, \lambda_{i}\right)=\left.\frac{\lambda_{i}^{*}}{O^{*}} \frac{\partial O}{\partial \lambda_{i}}\right|_{O=O^{*}}
$$

quantifies this sensitivity [63]. This particular value of $O$ is fine tuned if the sensitivity to $\lambda_{i}$ is larger at $O=O^{*}$ than at other values of $O$ [64]. If there are values of $O$ for which the sensitivity to $\lambda_{i}$ is small, then it is probably sufficient to use $c\left(O, \lambda_{i}\right)$ as the measure of fine tuning.

The function $c\left(m_{Z}^{2}, \lambda_{i}\right)$ is determined by performing the following. The sparticle spectrum in the minimal LEGM model is determined by the four parameters $\Lambda_{m e s s}, \mu_{3}^{2}, \mu$, and $\tan \beta^{5}$. The scale $\Lambda_{\text {mess }}$ fixes the boundary condition for the soft scalar masses, and an implicit dependence on $\tan \beta$ from $\lambda_{t}, \lambda_{b}$ and $\lambda_{\tau}$ arises in RG scaling ${ }^{6}$ from $\mu_{R G}=\Lambda_{\text {mess }}$ to the weak scale, that is chosen to be $\mu_{R G}^{2}=m_{t}^{2}+\frac{1}{2}\left(\tilde{m}_{t}^{2}+\tilde{m}_{t^{c}}^{2}\right)$. The extremization conditions of the scalar potential (Eqns.(5.10) and (5.11)) together with $m_{Z}$ and $m_{t}$ leave two free parameters that are chosen to be $\Lambda_{\text {mess }}$ and $\tan \beta$ (see Appendix A for the expressions for these functions).

A numerical analysis yields the value of $c\left(m_{Z}^{2}, \mu^{2}\right)$ that is displayed in Figure 5.1 in the $\left(\tan \beta, \Lambda_{\text {mess }}\right)$ plane. Note that $c\left(m_{Z}^{2}, \mu^{2}\right)$ is large throughout most of the parameter space, except for the region where $\tan \beta \gtrsim 5$ and the messenger scale is low. A strong constraint on a lower limit for $\Lambda_{\text {mess }}$ is from the righthanded selectron mass. Contours $m_{\tilde{e}_{R}}=75 \mathrm{GeV}(\sim$ the LEP limit from the

\footnotetext{
${ }^{5}$ Here an arbitrary $\mu_{3}^{2}$ at $\Lambda_{\text {mess }}$ is allowed for.

${ }^{6}$ The RG scaling of $\lambda_{t}$ was neglected.
} 
run at $\sqrt{s} \approx 170 \mathrm{GeV}[78])$ and $85 \mathrm{GeV}(\sim$ the ultimate LEP2 limit [79]) are also plotted. The (approximate) limit on the neutralino masses from the LEP run at $\sqrt{s} \approx 170 \mathrm{GeV}, m_{\chi_{1}^{0}}+m_{\chi_{2}^{0}}=160 \mathrm{GeV}$ and the ultimate LEP2 limit; $m_{\chi_{1}^{0}}+m_{\chi_{2}^{0}} \sim 180 \mathrm{GeV}$ are also shown in Figures 5.1a and 5.1c for $\operatorname{sgn}(\mu)=-1$ and Figures $5.1 \mathrm{~b}$ and $5.1 \mathrm{~d}$ for $\operatorname{sgn}(\mu)=+1$. The constraints from the present and the ultimate LEP2 limits on the chargino mass are weaker than or comparable to those from the selectron and the neutralino masses and are therefore not shown. If $m_{Z}$ were much larger, then $c \sim 1$. For example, with $m_{Z}=275 \mathrm{GeV}(550 \mathrm{GeV})$ and $\Lambda_{m e s s}=50(100) \mathrm{TeV}, c\left(m_{Z}^{2} ; \mu^{2}\right)$ varies between 1 and 5 for $1.4 \lessgtr \tan \beta \lessgtr 2$, and is $\approx 1$ for $\tan \beta>2$. This suggests that the interpretation that a large value for $c\left(m_{Z}^{2} ; \mu^{2}\right)$ implies that $m_{Z}$ is fine tuned is probably correct.

From Figure 5.1 it is concluded that in the minimal LEGM model a fine tuning of approximately $7 \%$ in the Higgs potential is required to produce the correct value for $m_{Z}$ if no sparticles are discovered at LEP2. Further, for this fine tuning the parameters of the model are restricted to the region $\tan \beta \gtrsim 5$ and $\Lambda_{\text {mess }} \approx 45$ $\mathrm{TeV}$, corresponding to $m_{\tilde{e}_{R}} \approx 85 \mathrm{GeV}$. It has also been checked that adding more complete $(5+\overline{5})$ 's does not reduce the fine tuning. 


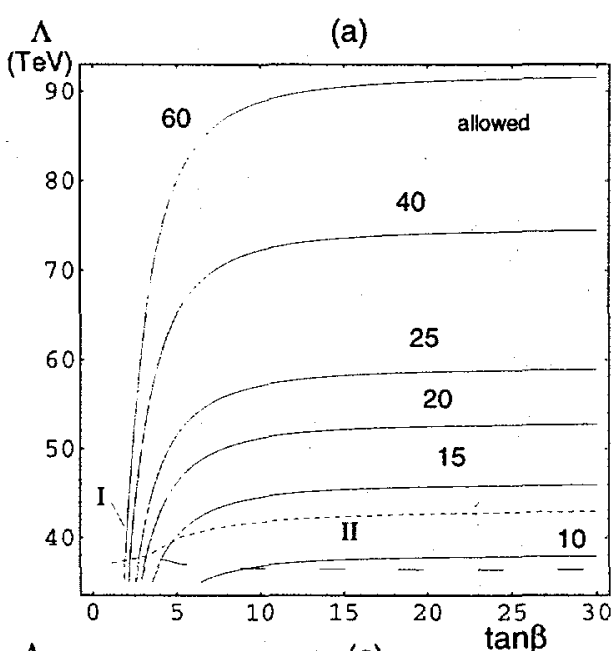

$\Lambda$

(b)
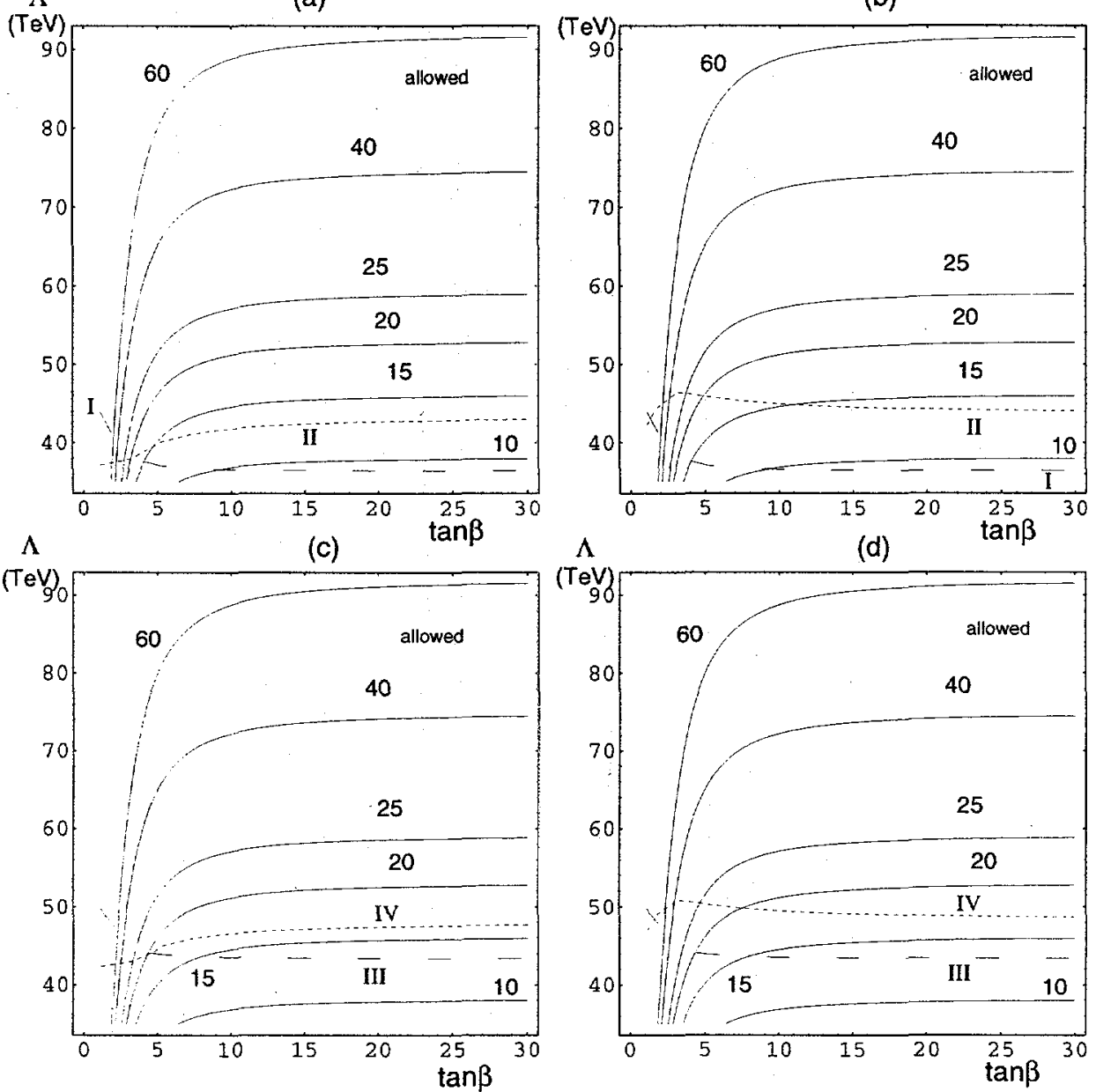

Figure 1

Figure 5.1: Contours of $c\left(m_{Z}^{2} ; \mu^{2}\right)=(10,15,20,25,40,60)$ for a MSSM with a messenger particle content of one $(5+\overline{5})$. In figures $(a)$ and $(c) \operatorname{sgn}(\mu)=-1$ and in figures $(b)$ and $(d) \operatorname{sgn}(\mu)=+1$. The constraints considered are: (I) $m_{\tilde{e}_{R}}=75$ $\mathrm{GeV}$, (II) $m_{\tilde{\chi}_{1}^{0}}+m_{\tilde{\chi}_{2}^{0}}=160 \mathrm{GeV}$, (III) $m_{\tilde{e}_{R}}=85 \mathrm{GeV}$, and (IV) $m_{\tilde{\chi}_{1}^{0}}+m_{\tilde{\chi}_{2}^{0}}=180$ $\mathrm{GeV}$. 


\subsection{A Toy Model to Reduce Fine Tuning}

\subsubsection{Model}

In this section the particle content and couplings in the messenger sector that are sufficient to reduce $\left|m_{H_{u}}^{2}\right|$ is discussed. The aim is to reduce $m_{\tilde{Q}}^{2} / m_{H_{u}}^{2}$ at the scale $\Lambda_{m e s s}$

The idea is to increase the number of messenger leptons $(S U(2)$ doublets) relative to the number of messenger quarks $(S U(3)$ triplets). This reduces both $m_{\tilde{Q}}^{2} / m_{H_{u}}^{2}$ and $m_{\tilde{Q}^{2}}^{2} / m_{\tilde{e}_{R}}^{2}$ at the scale $\Lambda_{m e s s}$ (see Eqn.(5.4)). This leads to a smaller value of $\left|m_{H_{u}}^{2}\right|$ in the RG scaling (see Eqn.(5.13)) and the scale $\Lambda_{m e s s}$ can be lowered since $m_{\tilde{e}_{R}}$ is larger. For example, three doublets and one triplet at a scale $\Lambda_{\text {mess }}=30 \mathrm{TeV}$, so that $m_{\tilde{e}_{R}} \approx 85 \mathrm{GeV}$ gives $\left|m_{H_{u}}^{2}\left(m_{\tilde{Q}}\right)\right| \approx(100 \mathrm{GeV})^{2}$ for $\lambda_{t}=1$.

This may be achieved by the following superpotential in the messenger sector

$$
\begin{aligned}
W= & \lambda_{q_{1}} S q_{1} \overline{q_{1}}+\lambda_{l_{1}} S l_{1} \overline{l_{1}}+\lambda_{l_{2}} S l_{2} \overline{l_{2}}+\lambda_{l_{3}} S l_{3} \overline{l_{3}}+\frac{1}{3} \lambda_{S} S^{3} \\
& +\lambda_{\phi} S \phi_{-} \phi_{+}+\frac{1}{3} \lambda_{N} N^{3}+\lambda_{q_{2}} N q_{2} \overline{q_{2}}+\lambda_{q_{3}} N q_{3} \overline{q_{3}}
\end{aligned}
$$

where $N$ is a gauge singlet. The two pairs of triplets $q_{2}, \bar{q}_{2}$ anci $q_{3}, \bar{q}_{3}$ are required at low energies to maintain gauge coupling unification. In this model the additional leptons $l_{2}, \bar{l}_{2}$ and $l_{3}, \bar{l}_{3}$ couple to the singlet $S$, whereas the additional quarks couple to a different singlet $N$ that does not couple to the messenger fields $\phi_{+}, \phi_{-}$. This can be enforced by discrete symmetries (such a model is discussed in section 5.7). Further, discrete charges that forbid any couplings between $N$ and $S$ at the 
renormalizable level are assumed to exist (this is true of the model in section 5.7) so that SUSY breaking is communicated first to $S$ and to $N$ only at a higher loop level.

\subsubsection{Mass Spectrum}

Before quantifying the fine tuning in this model, the mass spectrum of the additional states is briefly discussed. While these fields form complete representations of $S U(5)$, they are not degenerate in mass. The vev and $F$-component of the singlet $S$ gives a mass $\Lambda_{\text {mess }}$ to the messenger lepton multiplets if the $F$-term splitting between the scalars is neglected. As the squarks in $q_{i}+\bar{q}_{i}$ $(i=2,3)$ do not couple to $S$, they acquire a soft scalar mass from the same twoloop diagrams that are responsible for the masses of the MSSM squarks, yielding $m_{\tilde{q}} \approx \alpha_{3}\left(\Lambda_{m e s s}\right) \Lambda_{S U S Y} /(\sqrt{6} \pi)$. The fermions in $q+\bar{q}$ also acquire mass at this scale since, if either $\lambda_{q_{2}}$ or $\lambda_{q_{3}} \sim O(1)$, a negative value for $m_{N}^{2}$ (the soft scalar mass squared of $N$ ) is generated from the $\lambda_{q} N q \bar{q}$ coupling at one loop and thus a vev for $N \sim m_{\tilde{q}}$ is generated. The result is $m_{l} / m_{q} \approx \sqrt{6} \pi / \alpha_{3}\left(\Lambda_{\text {mess }}\right)\left(\Lambda_{\text {mess }} / \Lambda_{S U S Y}\right) \approx 85$

The mass splitting in the extra fields introduces a threshold correction to $\sin ^{2} \theta_{W}$ if it is assumed that the gauge couplings unify at some high scale $M_{G U T} \approx 10^{16} \mathrm{GeV}$. This splitting shifts the prediction for $\sin ^{2} \theta_{W}$ by an amount $\approx-7 \times 10^{-4} \ln \left(m_{l} / m_{q}\right) n$, where $n$ is the number of split $(5+\overline{5}) \cdot{ }^{7}$ In this case $n=2$

\footnotetext{
${ }^{7}$ The complete $(5+\overline{5})$, i.e., $l_{1}, \bar{l}_{1}$ and $q_{1}, \bar{q}_{1}$, that couples to $S$ is also split because $\lambda_{l} \neq \lambda_{q}$ at the messenger scale due to RG scaling from $M_{G U T}$ to $\Lambda_{m e s s}$. This splitting is small and
} 
and $m_{l} / m_{q} \sim 85$, so $\Delta \sin ^{2} \theta_{W} \sim-6 \times 10^{-3}$. If $\alpha_{3}\left(M_{Z}\right)$ and $\alpha_{e m}\left(M_{Z}\right)$ are used as. input, then using the two-loop RG equations $\sin ^{2} \theta_{W}(\overline{M S})=0.233 \pm O\left(10^{-3}\right)$ is predicted in a minimal SUSY-GUT [80]. The error is a combination of weak scale SUSY and GUT threshold corrections[80]. The central value of the theoretical prediction is a few percent higher than the measured value of $\sin ^{2} \theta_{W}(\overline{M S})=0.231 \pm 0.0003[36]$. The split extra fields shift the prediction of $\sin ^{2} \theta_{W}$ to $\sim 0.227 \pm O\left(10^{-3}\right)$ which is a few percent lower than the experimental value. In sections 5.6 and 5.7 it is shown that this spectrum is derivable from a $S U(5) \times S U(5)$ GUT in which the GUT threshold corrections to $\sin ^{2} \theta_{W}$ could be $\sim O\left(10^{-3}\right)-O\left(10^{-2}\right)[81]$. It is possible that the combination of these GUT threshold corrections and the split extra field threshold corrections make the prediction of $\sin ^{2} \theta_{W}$ more consistent with the observed value.

\subsubsection{Fine Tuning}

To quantify the fine tuning in these class of models the analysis of section 5.3 is applied. In the RG analysis the RG scaling of $\lambda_{t}$, the effect of the extra vector-like triplets on the RG scaling of the gauge couplings, and weak scale SUSY threshold corrections were neglected. That this approximation is consistent has been checked a postiori. As in section 5.3, the two free parameters are chosen to be $\Lambda_{\text {mess }}$ and $\tan \beta$. Contours of constant $c\left(m_{Z}^{2}, \mu^{2}\right)$ are presented in Figure 5.2. Shown are contours of $m_{\chi_{1}^{0}}+m_{\chi_{2}^{0}}=160 \mathrm{GeV}$, and $m_{\tilde{e}_{R}}=75 \mathrm{GeV}$ in Figure neglected. 
5.2a for $\operatorname{sgn}(\mu)=-1$, and $5.2 \mathrm{~b}$ for $\operatorname{sgn}(\mu)=+1$. These are roughly the present limits from LEP (including the run at $\sqrt{s} \approx 170 \mathrm{GeV}[78]$ ). The (approximate) ultimate LEP2 reaches [79] $m_{\chi_{1}^{0}}+m_{\chi_{2}^{0}}=180 \mathrm{GeV}$, and $m_{\tilde{e}_{R}}=85 \mathrm{GeV}$ are shown in figure $5.2 \mathrm{c}$ for $\operatorname{sgn}(\mu)=-1$ and figure $5.2 \mathrm{~d}$ for $\operatorname{sgn}(\mu)=+1$. Since $\mu^{2}(\approx(100$ $\mathrm{GeV})^{2}$ ) is much smaller in these models than in the minimal LEGM model, the neutralinos $\left(\chi_{1}^{0}\right.$ and $\left.\chi_{2}^{0}\right)$ are lighter so that the neutralino masses provide a stronger constraint on $\Lambda_{\text {mess }}$ than does the slepton mass limit. The chargino constraints are comparable to the neutralino constraints and are thus not shown. It is clear that there are areas of parameter space in which the fine tuning is improved to $\sim$ $40 \%$ (see figure 5.2 ).

While this model improves the fine tuning required of the $\mu$ parameter, it would be unsatisfactory if further fine tunings were required in other sectors of the model, for example, the sensitivity of $m_{Z}^{2}$ to $\mu_{3}^{2}, \Lambda_{\text {mess }}$ and $\lambda_{t}$ and the sensitivity of $m_{t}$ to $\mu^{2}, \mu_{3}^{2}, \Lambda_{m e s s}$ and $\lambda_{t}$. These are all found to be less than or comparable to $c\left(m_{Z}^{2} ; \mu^{2}\right)$. The other fine tunings are now discussed in detail.

For large $\tan \beta$, the sensitivity of $m_{Z}^{2}$ to $\mu_{3}^{2}, c\left(m_{Z}^{2} ; \mu_{3}^{2}\right) \propto 1 / \tan ^{2} \beta$, and is therefore smaller than $c\left(m_{Z}^{2} ; \mu^{2}\right)$. A numerical analysis shows that $c\left(m_{Z}^{2} ; \mu_{3}^{2}\right) \lesssim c\left(m_{Z}^{2} ; \mu^{2}\right)$ for all $\tan \beta$.

In the one-loop approximation $m_{H_{u}}^{2}$ and $m_{H_{d}}^{2}$ at the weak scale are proportional to $\Lambda_{m e s s}^{2}$ since all the soft masses scale with $\Lambda_{m e s s}$ and there is only a weak logarithmic dependence on $\Lambda_{\text {mess }}$ through the gauge couplings. It is found numerically that $\left(\Lambda_{\text {mess }}^{2} / m_{H_{u}}^{2}\right)\left(\partial m_{H_{u}}^{2} / \partial \Lambda_{\text {mess }}^{2}\right) \sim 1$. Then, $c\left(m_{Z}^{2} ; \Lambda_{\text {mess }}^{2}\right) \approx$ 


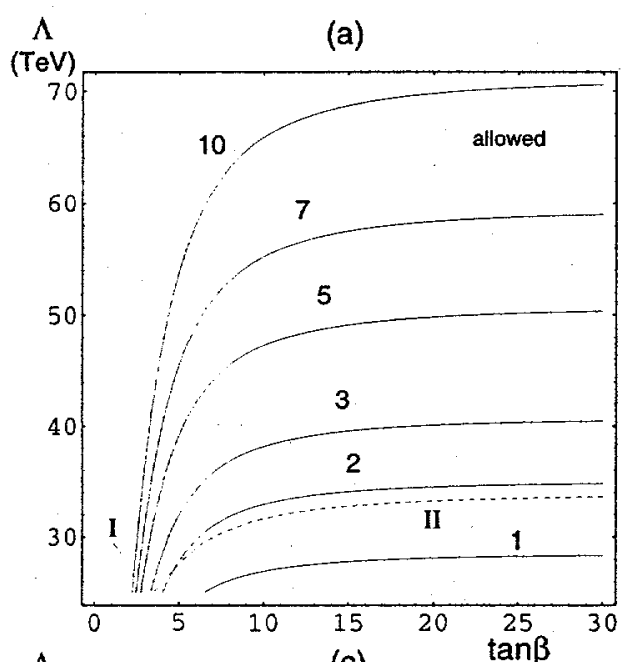

$\Lambda$
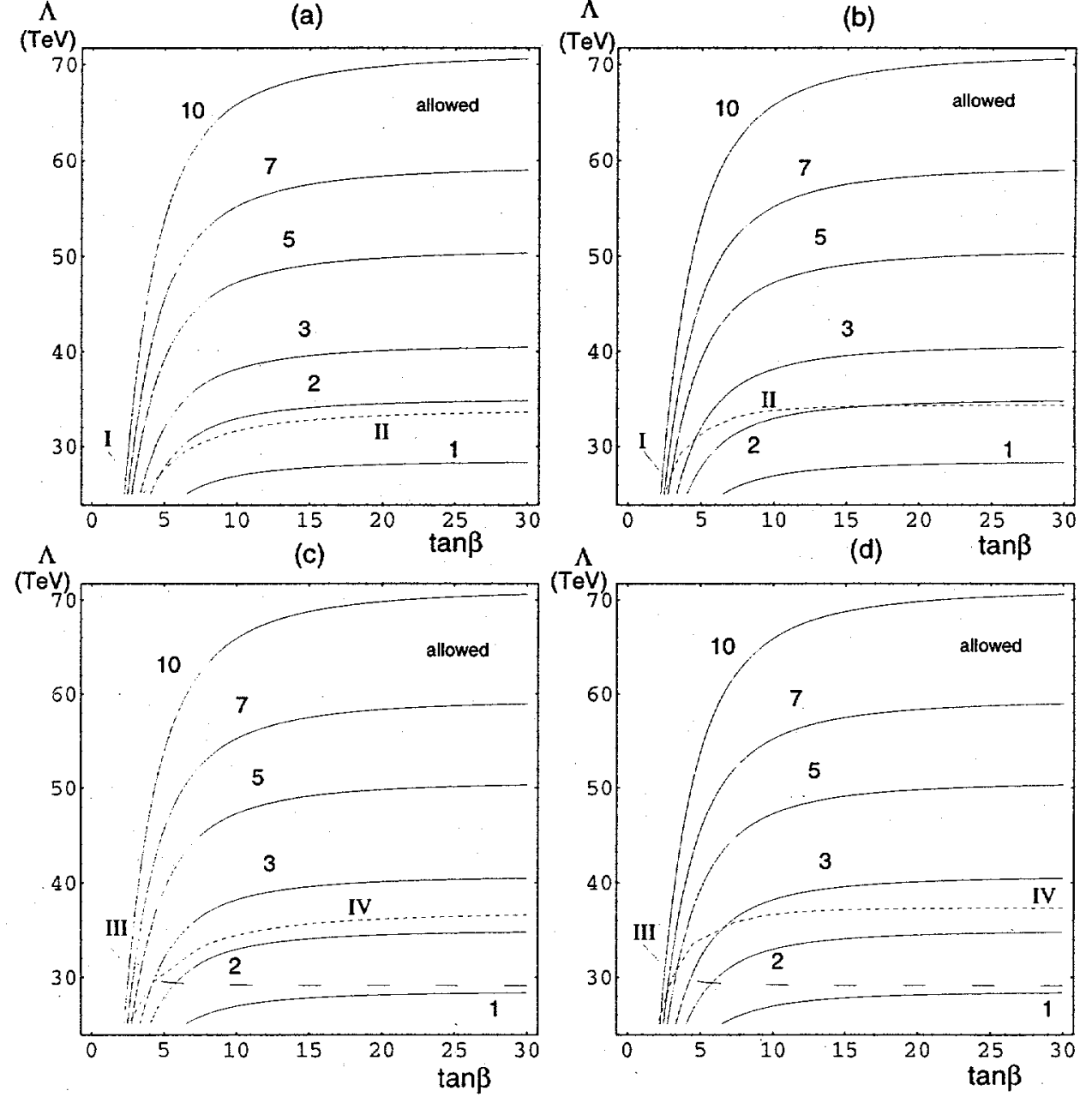

Figure 2

Figure 5.2: Contours of $c\left(m_{Z}^{2} ; \mu^{2}\right)=(1,2,3,5,7,10)$ for a MSSM with a messenger particle content of three $(l+\bar{l})^{\text {'s }}$ and one $(q+\bar{q})$. In figures $(a)$ and $(c) \operatorname{sgn}(\mu)=$ -1 and in figures $(b)$ and $(d) \operatorname{sgn}(\mu)=+1$. The constraints considered are: (I) $m_{\tilde{e}_{R}}=75 \mathrm{GeV}$, (II) $m_{\tilde{\chi}_{1}^{0}}+m_{\tilde{\chi}_{2}^{0}}=160 \mathrm{GeV}$, (III) $m_{\tilde{e}_{R}}=85 \mathrm{GeV}$, and (IV) $m_{\tilde{\chi}_{1}^{0}}+m_{\tilde{\chi}_{2}^{0}}=180 \mathrm{GeV}$ 
$c\left(m_{Z}^{2} ; m_{H_{d}}^{2}\right)+c\left(m_{Z}^{2} ; m_{H_{u}}^{2}\right)$. It is also found that $c\left(m_{Z}^{2} ; \Lambda_{m e s s}^{2}\right) \approx c\left(m_{Z}^{2} ; \mu^{2}\right)+1$ over most of the parameter space.

In the one-loop approximation, $m_{H_{u}}^{2}(t)$ is

$$
m_{H_{u}}^{2}(t) \approx m_{H_{u}}^{2}(0)+\left(m_{\tilde{Q}_{3}}^{2}(0)+m_{\tilde{u}_{3}^{c}}^{2}(0)+m_{H_{u}}^{2}(0)\right)\left(e^{-\frac{3 \lambda_{t}^{2}}{8 \pi^{2}} t}-1\right)
$$

Then, using $t \approx \ln \left(\Lambda_{m e s s} / m_{\bar{Q}_{3}}\right) \approx \ln \left(\sqrt{6} \pi / \alpha_{3}\right) \approx 4.5$ and $\lambda_{t} \approx 1, c\left(m_{Z}^{2} ; \lambda_{t}\right)$ is (see Appendix A)

$$
c\left(m_{Z}^{2} ; \lambda_{t}\right) \approx \frac{4}{m_{Z}^{2}} \frac{\partial m_{H_{u}}^{2}(t)}{\partial \lambda_{t}^{2}} \approx 50 \frac{m_{\tilde{Q}_{3}}^{2}}{(600 \mathrm{GeV})^{2}}
$$

This result measures the sensitivity of $m_{Z}^{2}$ to the value of $\lambda_{t}$ at the electroweak scale. While this sensitivity is large, it does not reflect the fact that $\lambda_{t}\left(M_{p l}\right)$ is the fundamental parameter of the theory, rather than $\lambda_{t}\left(M_{w e a k}\right)$. Using both numerical and analytic computations it is found that, for this model with three $(5+\overline{5})$ 's in addition to the MSSM particle content, $\delta \lambda_{t}\left(M_{w e a k}\right) \approx 0.1 \times \delta \lambda_{t}\left(M_{p l}\right)$, and therefore

$$
c\left(m_{Z}^{2} ; \lambda_{t}\left(M_{p l}\right)\right) \approx 5 \frac{m_{\tilde{Q}_{3}}^{2}}{(600 \mathrm{GeV})^{2}}
$$

For a scale of $\Lambda_{\text {mess }}=50 \mathrm{TeV}\left(m_{\tilde{Q}} \approx 600 \mathrm{GeV}\right), c\left(m_{Z}^{2} ; \lambda_{t}\left(M_{p l}\right)\right)$ is comparable to $c\left(m_{Z}^{2} ; \mu^{2}\right)$ which is $\approx 4$ to 5 . At a lower messenger scale, $\Lambda_{\text {mess }} \approx 35 \mathrm{TeV}$, corresponding to squark masses of $\approx 450 \mathrm{GeV}$, the sensitivity of $m_{Z}^{2}$ to $\lambda_{t}\left(M_{p l}\right)$ is $\approx 2.8$. This is comparable to $c\left(m_{Z}^{2} ; \mu^{2}\right)$ evaluated at the same scale.

The sensitivity of $m_{t}$ to the fundamental parameters is discussed next. Since, 


\begin{tabular}{|c|c|c|}
\hline \multirow[t]{3}{*}{687} & 616 & 12 \\
\hline & $m_{\tilde{Q}_{3}}$ & $m_{\tilde{u}_{3}^{c}}$ \\
\hline & 656 & 546 \\
\hline
\end{tabular}

Table 5.1: Soft scalar masses in $\mathrm{GeV}$ for messenger particle content of three $(l+\bar{l})^{\text {'s }}$ and one $q+\bar{q}$ and a scale $\Lambda_{\text {mess }}=50 \mathrm{TeV}$.

$m_{t}^{2}=\frac{1}{2} v^{2} \sin ^{2} \beta \lambda_{t}^{2}$

$$
c\left(m_{t} ; \lambda_{i}\right)=\delta_{\lambda_{t} \lambda_{i}}+\frac{1}{2} c\left(m_{Z}^{2} ; \lambda_{i}\right)+\frac{\cos ^{3} \beta}{\sin \beta} \frac{\partial \tan \beta}{\partial \lambda_{i}} \lambda_{i}
$$

is obtained. Numerical computations determine that the last term in $c\left(m_{t} ; \lambda_{i}\right)$ is small compared to $c\left(m_{Z}^{2} ; \lambda_{i}\right)$ and thus over most of parameter space $c\left(m_{t} ; \lambda_{i}\right) \approx$ $\frac{1}{2} c\left(m_{Z}^{2} ; \lambda_{i}\right)$. As before, the sensitivity of $m_{t}$ to the value of $\lambda_{t}$ at the GUT/Planck scale is much smaller than the sensitivity to the value of $\lambda_{t}$ at the weak scale.

\subsubsection{Sparticle Spectrum}

The sparticle spectrum is now briefly discussed to highlight deviations from the mass relations predicted in the minimal LEGM model. For example, with three doublets and one triplet at a scale of $\Lambda=50 \mathrm{TeV}$, the soft scalar masses (in $\mathrm{GeV}$ ) at a renormalization scale $\mu_{R G}^{2}=m_{t}^{2}+\frac{1}{2}\left(m_{\tilde{Q}_{3}}^{2}+m_{\tilde{u}_{3}^{c}}^{2}\right) \approx(630 \mathrm{GeV})^{2}$, for $\lambda_{t}=1$, are shown in Table 5.1 .

Two observations that are generic to this type of model are: (i) By construc- 
tion, the spread in the soft scalar masses is less than in the minimal LEGM model. (ii) The gaugino masses do not satisfy the one-loop SUSY-GUT relation $M_{i} / \alpha_{i}=$ constant. In this case, for example, $M_{3} / \alpha_{3}: M_{2} / \alpha_{2} \approx 1: 3$ and $M_{3} / \alpha_{3}: M_{1} / \alpha_{1} \approx$ 5:11 to one-loop.

It is also found that for $\tan \beta \gtrsim 3$, the Next Lightest Supersymmetric Particle (NLSP) is one of the neutralinos, whereas for $\tan \beta \lesssim 3$, the NLSP is the righthanded stau. Further, for these small values of $\tan \beta$, the three right-handed sleptons are degenerate to within $\approx 200 \mathrm{MeV}$.

\section{$5.5 \quad$ NMSSM}

In section 5.3 , the $\mu$ term and the SUSY breaking mass $\mu_{3}^{2}$ were put in by hand. There it was found that these parameters had to be fine tuned in order to correctly reproduce the observed $Z$ mass. The extent to which this is a "problem" may only be evaluated within a specific model that generates both the $\mu$ and $\mu_{3}^{2}$ terms.

For this reason, in this section a possible way to generate both the $\mu$ term and $\mu_{3}^{2}$ term in a manner that requires a minimal modification to the model of either section 5.2 or section 5.4 is discussed. The easiest way to generate these mass terms is to introduce a singlet $N$ and add the interaction $N H_{u} H_{d}$ to the superpotential (the NMSSM)[72]. The vev of the scalar component of $N$ generates $\mu$ and the vev of the $F$-component of $N$ generates $\mu_{3}^{2}$.

Note that for the "toy model" solution to the fine tuning problem (section 
$5.4)$, the introduction of the singlet occurs at no additional cost. Recall that in that model it was necessary to introduce a singlet $N$, distinct from $S$, such that the vev of $N$ gives mass to the extra light vector-like triplets, $q_{i}, \vec{q}_{i}(i=2,3)$ (see equation 5.15). Further, discrete symmetries (see section 5.7) are imposed to isolate $N$ from SUSY breaking in the messenger sector. This last requirement is necessary to solve the fine tuning problem: if both the scalar and $F$-component of $N$ acquired a vev at the same scale as $S$, then the extra triplets that couple to $N$ would also act as messenger fields. In this case the messenger fields would form complete $(5+\overline{5})$ 's and the fine tuning problem would be reintroduced. With $N$ isolated from the messenger sector at tree level, a vev for $N$ at the electroweak scale is naturally generated, as discussed in section 5.4.

Next, a comment on the necessity and origin of these extra triplets is made. Recall that in the toy model of section 5.4 these triplets were required to maintain the SUSY-GUT prediction for $\sin ^{2} \theta_{W}$. Further, it will be seen that they are required in order to generate a large enough $-m_{N}^{2}$ (the soft scalar mass squared of the singlet $N$ ). Finally, in the GUT model of section 5.7, the lightness of these triplets (as compared to the missing doublets) is the consequence of a doublettriplet splitting mẹchanism.

The superpotential in the electroweak symmetry breaking sector is

$$
W=\frac{\lambda_{N}}{3} N^{3}+\lambda_{q} N q \bar{q}-\lambda_{H} N H_{u} H_{d}
$$

which is similar to an NMSSM except for the coupling of $N$ to the triplets. The 
superpotential in the messenger sector is given by Eqn.(5.15).

The scalar potential is ${ }^{8}$

$$
\begin{aligned}
\dot{V}= & \sum_{i}\left|F_{i}\right|^{2}+m_{N}^{2}|N|^{2}+m_{H_{u}}^{2}\left|H_{u}\right|^{2}+m_{H_{d}}^{2}\left|H_{d}\right|^{2}+\text { D-terms } \\
& -\left(A_{H} N H_{u} H_{d}+\text { h.c. }\right)+V_{1-\text { loop } .}
\end{aligned}
$$

The extremization conditions for the vevs of the real components of $N, H_{u}$ and $H_{d}$, denoted by $v_{N}, v_{u}$ and $v_{d}$ respectively (with $v=\sqrt{v_{u}^{2}+v_{d}^{2}} \approx 250 \mathrm{GeV}$ ), are

$$
\begin{aligned}
v_{N}\left(\tilde{m}_{N}^{2}+\lambda_{H}^{2} \frac{v^{2}}{2}+\lambda_{N}^{2} v_{N}^{2}-\lambda_{H} \lambda_{N} v_{u} v_{d}\right)-\frac{1}{\sqrt{2}} A_{H} v_{u} v_{d}=0 \\
\frac{1}{2} m_{Z}^{2}=\frac{\tilde{\mu}_{1}^{2}-\tilde{\mu}_{2}^{2} \tan ^{2} \beta}{\tan ^{2} \beta-1} \\
\sin 2 \beta=2 \frac{\mu_{3}^{2}}{\tilde{\mu}_{2}^{2}+\tilde{\mu}_{1}^{2}}
\end{aligned}
$$

with

$$
\begin{aligned}
\mu^{2} & =\frac{1}{2} \lambda_{H}^{2} v_{N}^{2} \\
\mu_{3}^{2} & =-\frac{1}{2} \lambda_{H}^{2} v_{u} v_{d}+\frac{1}{2} \lambda_{H} \lambda_{N} v_{N}^{2}+A_{H} \frac{1}{\sqrt{2}} v_{N} \\
\tilde{m}_{i}^{2} & =m_{i}^{2}+2 \frac{\partial V_{1-\text { loop }}}{\partial v_{i}^{2}} ; i=(u, d, N)
\end{aligned}
$$

The expected size of the Yukawa couplings $\lambda_{q}, \lambda_{N}$ and $\lambda_{H}$ is now discussed. The RGE's must be used to evolve these couplings from their values at $M_{G U T}$ or $M_{p l}$ to the weak scale. The quarks and the Higgs doublets receive wavefunction

\footnotetext{
${ }^{8}$ In models of gauge mediated SUSY breaking, $A_{H}=0$ at tree-level and a non-zero value of $A_{H}$ is generated at one loop. The trilinear scalar term $A_{N} N^{3}$ is generated at two-loops and is neglected.
} 
renormalization from $S U(3)$ and $S U(2)$ gauge interactions respectively, whereas the singlet $N$ does not receive at one-loop any wavefunction renormalization from gauge interactions. So, the couplings at the weak scale are in the order: $\lambda_{q} \sim$ $O(1)>\lambda_{H}>\lambda_{N}$ if they all are $O(1)$ at the GUT/Planck scale.

It is next shown that without the $N q \bar{q}$ coupling it is difficult to drive a vev for $N$. The one loop RGE for $m_{N}^{2}$ is

$$
\frac{d m_{N}^{2}}{d t} \approx \frac{6 \lambda_{N}^{2}}{8 \pi^{2}} m_{N}^{2}(t)+\frac{2 \lambda_{H}^{2}}{8 \pi^{2}}\left(m_{H_{u}}^{2}(t)+m_{H_{d}}^{2}(t)+m_{N}^{2}(t)\right)+\frac{3 \lambda_{q}^{2}}{8 \pi^{2}}\left(m_{\tilde{q}}^{2}(t)+m_{\overline{\bar{q}}}^{2}(t)\right) .
$$

Since $N$ is a gauge-singlet, $m_{N}^{2}=0$ at $\Lambda_{\text {mess }}$. Further, if $\lambda_{q}=0$, an estimate for $m_{N}^{2}$ at the weak scale is then

$$
m_{N}^{2} \approx-\frac{2 \lambda_{H}^{2}}{8 \pi^{2}}\left(m_{H_{u}}^{2}(0)+m_{H_{d}}^{2}(0)\right) \ln \left(\frac{\Lambda_{\text {mess }}}{m_{H_{d}}}\right)
$$

i.e., $\lambda_{H}$ drives $m_{N}^{2}$ negative. The extremization condition for $v_{N}$, Eqn.(5.22), and using Eqns.(5.24) and (5.26) (neglecting $A_{H}$ ) shows that

$$
m_{N}^{2}+\lambda_{H}^{2} \frac{v^{2}}{2} \approx \lambda_{H}^{2}\left(\frac{v^{2}}{2}-\frac{2}{8 \pi^{2}}\left(m_{H_{u}}^{2}(0)+m_{H_{d}}^{2}(0)\right) \ln \left(\frac{\Lambda_{m e s s}}{m_{H_{d}}}\right)\right)
$$

has to be negative for $N$ to acquire a vev. This implies that $m_{H_{u}}^{2}$ and $m_{H_{d}}^{2}$ at $\Lambda_{\text {mess }}$ have to be greater than $\sim(350 \mathrm{GeV})^{2}$ which implies that a fine tuning of a few percent is required in the electroweak symmetry breaking sector. With $\lambda_{q} \sim O(1)$, however, there is an additional negative contribution to $m_{N}^{2}$ given approximately by

$$
-\frac{3 \lambda_{q}^{2}}{8 \pi^{2}}\left(m_{q}^{2}(0)+m_{\bar{q}}^{2}(0)\right) \ln \left(\frac{\Lambda_{m e s s}}{m_{\tilde{q}}}\right)
$$


This contribution dominates the one in Eqn.(5.29) since $\lambda_{q}>\lambda_{H}$ and the squarks $\tilde{q}, \tilde{\tilde{q}}$ have soft masses larger than the Higgs. Thus, with $\lambda_{q} \neq 0, m_{N}^{2}+\lambda_{H}^{2} v^{2} / 2$ is naturally negative.

Fixing $m_{Z}$ and $m_{t}$, leaves the following parameters : $\Lambda_{m e s s}, \lambda_{q}, \lambda_{H}, \lambda_{N}, \tan \beta$, and $v_{N}$. Three of the parameters are fixed by the three extremization conditions, leaving three free parameters that for convenience are chosen to be $\Lambda_{m e s s}, \tan \beta \geq 1$, and $\lambda_{H}$. The signs of the vevs are fixed to be positive by requiring a stable vacuum and no spontaneous $C P$ violation. The three extremization equations determine the following relations

$$
\begin{aligned}
\lambda_{N} & =\frac{2}{\lambda_{H} v_{N}^{2}}\left(\mu_{3}^{2}+\frac{1}{4} \lambda_{H}^{2} \sin 2 \beta v^{2}-\frac{1}{\sqrt{2}} A_{H} v_{N}\right), \\
v_{N} & =\sqrt{2} \frac{\mu}{\lambda_{H}} \\
\tilde{m}_{N}^{2} & =\lambda_{N} \lambda_{H} \frac{1}{2} \sin 2 \beta v^{2}-\lambda_{N}^{2} v_{N}^{2}-\frac{1}{2} \lambda_{H}^{2} v^{2}+\frac{1}{2 \sqrt{2}} A_{H} \sin 2 \beta \frac{v^{2}}{v_{N}},
\end{aligned}
$$

where

$$
\begin{aligned}
\mu^{2} & =-\frac{1}{2} m_{Z}^{2}+\frac{\tilde{m}_{H_{u}}^{2} \tan ^{2} \beta-\tilde{m}_{H_{d}}^{2}}{1-\tan ^{2} \beta}, \\
2 \mu_{3}^{2} & =\sin 2 \beta\left(2 \mu^{2}+\tilde{m}_{H_{u}}^{2}+\tilde{m}_{H_{d}}^{2}\right) .
\end{aligned}
$$

The superpotential term $N H_{u} H_{d}$ couples the RGE's for $m_{H_{u}}^{2}, m_{H_{d}}^{2}$ and $m_{N}^{2}$. Thus the values of these masses at the electroweak scale are, in general, complicated functions of the Yukawa parameters $\lambda_{t}, \lambda_{H}, \lambda_{N}$ and $\lambda_{q}$. In this case, two of these Yukawa parameters $\left(\lambda_{q}\right.$ and $\left.\lambda_{N}\right)$ are determined by the extremization equations and a closed form expression for the derived quantities cannot be found. To simplify the analysis, the dependence of $m_{H_{u}}^{2}$ and $m_{H_{d}}^{2}$ on $\lambda_{H}$ induced in RG scaling 
from $\dot{\Lambda}_{\text {mess }}$ to the weak scale is neglected. Then $m_{H_{u}}^{2}$ and $m_{H_{d}}^{2}$ depend only on $\Lambda_{m e s s}$ and $\tan \beta$ and thus closed form solutions for $\lambda_{N}, v_{N}$ and $\tilde{m}_{N}^{2}$ can be obtained using the above equations. Once $\tilde{m}_{N}^{2}$ at the weak scale is obtained, the value of $\lambda_{q}$ is obtained by using an approximate analytic solution. An exact numerical solution of the RGE's then shows that the above approximation is consistent.

\subsubsection{Fine Tuning and Phenomenology}

The fine tuning functions considered below are $c\left(O ; \lambda_{H}\right), c\left(O ; \lambda_{N}\right), c\left(O ; \lambda_{t}\right)$, $c\left(O ; \lambda_{q}\right)$ and $c\left(O ; \Lambda_{\text {mess }}\right)$ where $O$ is either $m_{Z}^{2}$ or $m_{t}$. The expressions for the fine tuning functions and other details are given in Appendix A. In the RG analysis the approximations discussed in subsection 5.4.3 and above were used and found to be consistent. Fine tuning contours of $c\left(m_{Z}^{2} ; \lambda_{H}\right)$ are displayed in Figures $5.3 \mathrm{a}$ and $5.3 \mathrm{~b}$ for $\lambda_{H}=0.1$ and Figures $5.3 \mathrm{c}$ and $5.3 \mathrm{~d}$ for $\lambda_{H}=0.5$. Numerical computations show that the other fine tuning functions are either smaller or comparable to $c\left(m_{Z}^{2} ; \lambda_{H}\right) \cdot{ }^{9}$

The existing phenomenological constraints on this model and also the ultimate constraints if LEP2 does not discover SUSY/light Higgs $(h)$ are discussed. These are shown in.Figures 5.3a, 5.3c and Figures 5.3b, 5.3d respectively. The processes

\footnotetext{
${ }^{9}$ In computing these functions the weak scale value of the couplings $\lambda_{N}$ and $\lambda_{H}$ has been used. But since $\lambda_{N}$ and $\lambda_{H}$ do not have a fixed point behavior, it is found that $\lambda_{H}\left(M_{G U T}\right) / \lambda_{H}\left(m_{Z}\right) \partial \lambda_{H}\left(m_{Z}\right) / \partial \lambda_{H}\left(M_{G U T}\right) \sim 1$ so that, for example, $c\left(m_{Z}^{2} ; \lambda_{H}\left(M_{G U T}\right)\right) \approx$ $c\left(m_{Z}^{2} ; \lambda_{H}\left(m_{Z}\right)\right)$.
} 

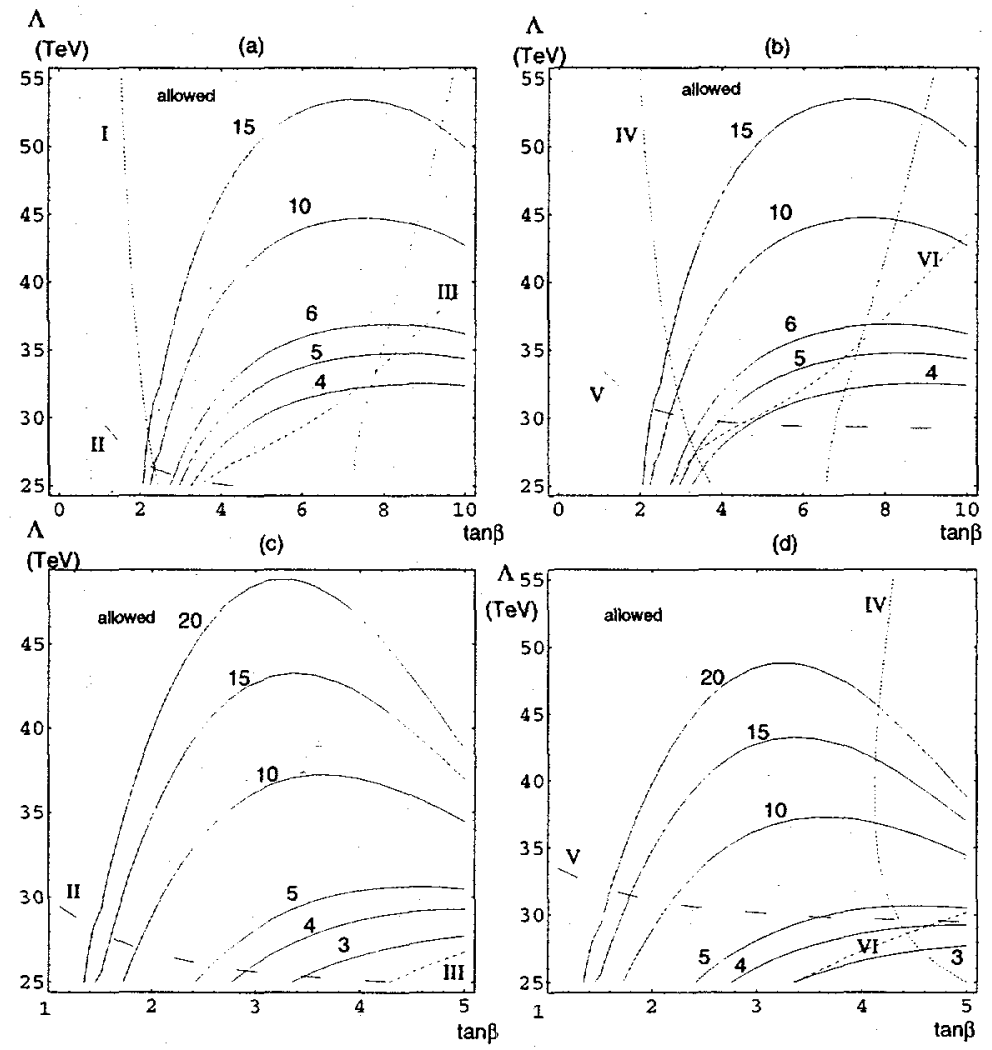

Figure 3

Figure 5.3: Contours of $c\left(m_{Z}^{2} ; \lambda_{H}\right)$ for the NMSSM of Section 3.5 and a messenger particle content of three $(l+\bar{l})$ 's and one $(q+\bar{q})$. In figures $(a)$ and $(b)$, $c\left(m_{Z}^{2} ; \lambda_{H}\right)=(4,5,6,10,15)$ and $\lambda_{H}=0.1$. In figures $(c)$ and $(d), c\left(m_{Z}^{2} ; \lambda_{H}\right)=(3$ $4,5,10,15,20)$ and $\lambda_{H}=0.5$. The constraints considered are: (I) $m_{h}+m_{a}=m_{Z}$, (II) $m_{\tilde{e}_{R}}=75 \mathrm{GeV}$, (III) $m_{\tilde{\chi}_{1}^{0}}+m_{\tilde{\chi}_{2}^{0}}=160 \mathrm{GeV}$, (IV) $m_{h}=92 \mathrm{GeV}$, (V) $m_{\tilde{e}_{R}}=85$ $\mathrm{GeV}$, and (VI) $m_{\tilde{\chi}_{1}^{0}}+m_{\tilde{\chi}_{2}^{0}}=180 \mathrm{GeV}$. For $\lambda_{H}=0.5$, the limit $m_{h}>70 \mathrm{GeV}$ constrains $\tan \beta<5$ (independent of $\Lambda_{\text {mess }}$ ) and is thus not shown. 
$e^{+} e^{-} \rightarrow Z h, e^{+} e^{-} \rightarrow(h+$ pseudoscalar $), e^{+} e^{-} \rightarrow \chi^{+} \chi^{-}, e^{+} e^{-} \rightarrow \chi_{1}^{0} \chi_{2}^{0}$, and $e^{+} e^{-} \rightarrow$ $\tilde{e}_{R} \tilde{e}_{R}^{*}$ observable at LEP are considered. Since this model also has a light pseudoscalar, the upsilon decays $\Upsilon \rightarrow(\gamma+$ pseudoscalar $)$ are also considered. It is found that the model is phenomenologically viable and requires a $\sim 20 \%$ tuning even if no new particles are discovered at LEP2.

The constraints on the scalar and pseudoscalar spectra of this model are first considered. There are three neutral scalars, two neutral pseudoscalars and one complex charged scalar. First consider the mass spectrum of the pseudoscalars. At the boundary scale $\Lambda_{\text {mess }}$, SUSY is softly broken in the visible sector only by the soft scalar masses and the gaugino masses. Further, the superpotential of Eqn.(5.20) has an $R$-symmetry. Therefore, at the tree level, i.e., with $A_{H}=0$, the scalar potential of the visible sector (equation 5.21) has a global symmetry. This symmetry is spontaneously broken by the vevs of $N^{R}, H_{u}^{R}$, and $H_{d}^{R}$ (the superscript $R$ denotes the real component of fields), so that one physical pseudoscalar is massless at tree level. It is

$$
a=\frac{1}{\sqrt{v_{N}^{2}+v^{2} \sin ^{2} 2 \beta}}\left(v_{N} N^{I}+v \sin 2 \beta \cos \beta H_{u}^{I}+v \sin 2 \beta \sin \beta H_{d}^{I}\right)
$$

where the superscripts $I$ denote the imaginary components of the fields. The second pseudoscalar,

$$
A \sim-\frac{2}{v_{N}} N^{I}+\frac{H_{u}^{I}}{v \sin \beta}+\frac{H_{d}^{I}}{v \cos \beta}
$$

acquires a mass

$$
m_{A}^{2}=\frac{1}{2} \lambda_{H} \lambda_{N} v_{N}^{2}(\tan \beta+\cot \beta)+\lambda_{H} \lambda_{N} v^{2} \sin 2 \beta
$$


through the $\left|F_{N}\right|^{2}$ term in the scalar potential.

The pseudoscalar $a$ acquires a mass once an $A_{H}$-term is generated, at one-loop, through interactions with the gauginos. Including only the wino contribution in the one-loop RGE, $A_{H}$ is given by

$$
\begin{aligned}
A_{H} & \approx 6 \frac{\alpha_{2}\left(\Lambda_{\text {mess }}\right)}{4 \pi} M_{2} \lambda_{H} \ln \left(\frac{\Lambda_{\text {mess }}}{M_{2}}\right), \\
& \approx 20 \lambda_{H}\left(\frac{M_{2}}{280 \mathrm{GeV}}\right) \mathrm{GeV},
\end{aligned}
$$

where $M_{2}$ is the wino mass at the weak scale. Neglecting the mass mixing between the two pseudoscalars, the mass of the pseudo-Nambu-Goldstone boson is computed to be

$$
\begin{aligned}
m_{a}^{2} & =\frac{9}{\sqrt{2}} A v_{N} v_{u} v_{d} /\left(v_{N}^{2}+v^{2} \sin ^{2} 2 \beta\right) \\
& \approx(40)^{2}\left(\frac{\lambda_{H}}{0.1}\right) \frac{M_{2}}{280 \mathrm{GeV}} \sin 2 \beta\left(\frac{\frac{v_{N}}{250 \mathrm{GeV}}}{\sin ^{2} 2 \beta+\left(\frac{v_{N}}{250 \mathrm{GeV}}\right)^{2}}\right)(\mathrm{GeV})^{2}
\end{aligned}
$$

If the mass of $a$ is less than $7.2 \mathrm{GeV}$, it could be detected in the decay $\Upsilon \rightarrow a+\gamma[36]$. Comparing the ratio of decay width for $\Upsilon \rightarrow a+\gamma$ to $\Upsilon \rightarrow \mu^{-}+\mu^{+}[36,82]$, the limit

$$
\frac{\sin 2 \beta \tan \beta}{\sqrt{\left(\frac{v_{N}}{250 \mathrm{GeV}}\right)^{2}+\sin ^{2} 2 \beta}}<0.43
$$

is found.

Further constraints on the spectra are obtained from collider searches. The non-detection of $Z \rightarrow$ scalar $+a$ at LEP implies that the combined mass of the lightest Higgs scalar and $a$ must exceed $\sim 92 \mathrm{GeV}$. Also, the process $e^{+} e^{-} \rightarrow Z h$ may be observable at LEP2. For $\lambda_{H}=0.1$, the constraint $m_{h}+m_{a} \gtrsim 92 \mathrm{GeV}$ is 
stronger than $m_{h} \gtrsim 70 \mathrm{GeV}$ which is the limit from LEP at $\sqrt{s} \approx 170 \mathrm{GeV}[78]$. The contour of $m_{h}+m_{a}=92 \mathrm{GeV}$ is shown in Figure 5.3a. Shown in Figure 5.3b is the contour of $m_{h}=92 \mathrm{GeV}$ ( $\sim$ the ultimate LEP2 reach [83]). For $\lambda_{H}=0.5$, the constraint $m_{h} \gtrsim 70 \mathrm{GeV}$ is stronger than $m_{h}+m_{a} \gtrsim 92 \mathrm{GeV}$ and restricts $\tan \beta \lesssim 5$ independent of $\Lambda_{\text {mess }}$. The contour $m_{h}=92 \mathrm{GeV}$ is shown in Figure 5.3d. Note that the allowed parameter space is not significantly constrained. These limits make the constraint of Eqn.(5.42) redundant. The left-right mixing between the two top squarks was neglected in computing the top squark radiative corrections to the Higgs masses.

The pseudo-Nambu-Goldstone boson $a$ might be produced along with the lightest scalar $h$ at LEP. The tree-level cross section in units of $R=87 / \mathrm{s} \mathrm{nb}$ is

$$
\sigma\left(e^{+} e^{-} \rightarrow h a\right) \approx 0.15 \frac{s^{2}}{\left(s-m_{Z}^{2}\right)^{2}} \lambda^{2} v\left(1, \frac{m_{h}^{2}}{s}, \frac{m_{a}^{2}}{s}\right)^{3}
$$

where $g \lambda / \cos \theta_{W}$ is the $Z(a \partial h-h \partial a)$ coupling, and

$$
\begin{gathered}
v(x, y, z)=\sqrt{(x-y-z)^{2}-4 y z} . \text { If } h=c_{N} N^{R}+c_{u} H_{u}^{R}+c_{d} H_{d}^{R}, \text { then } \\
\lambda=\sin 2 \beta \frac{\cos \beta c_{u}-\sin \beta c_{d}}{\sqrt{\left(\frac{v_{N}}{250 \mathrm{GeV}}\right)^{2}+\sin ^{2} 2 \beta}} .
\end{gathered}
$$

A numerical check of the parameter space allowed by $m_{h} \gtrsim 70 \mathrm{GeV}$ and $\lambda_{H} \leq 0.5$ shows that the production cross-section for $h a$ is less than both the current limit set by DELPHI [84] and a (possible) exclusion limit of $30 \mathrm{fb}[83]$ at $\sqrt{s} \approx 192 \mathrm{GeV}$. The production cross-section for $h A$ is larger than for $h a$ and $A$ is therefore in principle easier to detect. However, for the parameter space allowed by $m_{h} \gtrsim 70$ $\mathrm{GeV}$, numerical calculations show that $m_{A} \gtrsim 125 \mathrm{GeV}$, so that this channel is 
not kinematically accessible.

The charged Higgs mass is

$$
m_{H^{ \pm}}^{2}=m_{W}^{2}+m_{H_{u}}^{2}+m_{H_{d}}^{2}+2 \mu^{2}
$$

which is greater than about $200 \mathrm{GeV}$ in this model since $m_{H_{d}}^{2} \gtrsim(200 \mathrm{GeV})^{2}$ for $\Lambda_{m e s s} \gtrless 35 \mathrm{TeV}$ and as $\mu^{2} \sim-m_{H_{u}}^{2}$.

The neutralinos and charginos may be observable at LEP2 at $\sqrt{s} \approx 192 \mathrm{GeV}$ if $m_{\chi^{+}} \lesssim 95 \mathrm{GeV}$ and $m_{\chi_{1}^{0}}+m_{\chi_{2}^{0}} \lesssim 180 \mathrm{GeV}$. These two constraints are comparable, and thus only one of these is displayed in Figures $5.3 \mathrm{~b}$ and $5.3 \mathrm{~d}$, for $\lambda_{H}=0.1$ and $\lambda_{H}=0.5$ respectively. Also, contours of $m_{\chi_{1}^{0}}+m_{\chi_{2}^{0}}=160 \mathrm{GeV}(\sim$ the LEP kinematic limit at $\sqrt{s} \approx 170 \mathrm{GeV}$ ) are shown in Figures 5.3a and 5.3c. Contours of $85 \mathrm{GeV}(\sim$ the ultimate LEP2 limit) and $75 \mathrm{GeV}(\sim$ the LEP limit from $\sqrt{s} \approx 170$ $\mathrm{GeV}$ ) for the right-handed selectron mass further constrain the parameter space.

The results presented in all the figures are for a central value of $m_{t}=175 \mathrm{GeV}$. By varying the top quark mass by $10 \mathrm{GeV}$ about the central value of $m_{t}=175$ $\mathrm{GeV}$ the fine tuning measures and the LEP2 constraints (the Higgs mass and the neutralino masses) are found to vary by $\approx 30 \%$, but the qualitative features are unchanged.

From Figure 5.3 it is seen that there is parameter space allowed by the present limits in which the tuning is $\approx 30 \%$. Even if no new particles are discovered at $\mathrm{LEP} 2$, the tuning required for some region is $\approx 20 \%$.

It is also interesting to compare the fine tuning measures with those found 
in the minimal LEGM model (one messenger $(5+\overline{5})$ ) with an extra singlet $N$ to generate the $\mu$ and $\mu_{3}^{2}$ terms. ${ }^{10}$ In Figure 5.4 the fine tuning contours for $c\left(m_{Z}^{2} ; \lambda_{H}\right)$ are presented for $\lambda_{H}=0.1$. Contours of $m_{\tilde{e}_{R}}=75 \mathrm{GeV}$ and $m_{\chi_{1}^{0}}+m_{\chi_{2}^{0}}=160 \mathrm{GeV}$ are also shown in Figure 5.4a. For $\lambda_{H}=0.1$, the constraint $m_{h}+m_{a} \gtrsim 92 \mathrm{GeV}$ is stronger than the limit $m_{h} \gtrsim 70 \mathrm{GeV}$ and is shown in the Figure 5.4a. In Figure $5.4 \mathrm{~b}$, the (approximate) ultimate LEP2 limits are shown, i.e., $m_{h}=92 \mathrm{GeV}$, $m_{\chi_{1}^{0}}+m_{\chi_{2}^{0}}=180 \mathrm{GeV}$ and $m_{\tilde{e}_{R}}=85 \mathrm{GeV}$. Of these constraints, the bound on the lightest Higgs mass (either $m_{h}+m_{a} \gtrsim 92 \mathrm{GeV}$ or $m_{h} \gtrsim 92 \mathrm{GeV}$ ) provides a strong lower limit on the messenger scale. So in the parameter space allowed by present limits the fine tuning is $\lesssim 2 \%$ and if LEP2 does not discover new particles, the fine tuning will be $\lesssim 1 \%$. The coupling $\lambda_{H}$. is constrained to be not significantly larger than 0.1 if the constraint $m_{h}+m_{a} \gtrsim 92 \mathrm{GeV}$ (or $m_{h} \gtrsim 92 \mathrm{GeV}$ ) is imposed and if the fine tuning is required to be no worse than $1 \%$.

\subsection{Models Derived from a GUT}

This section discusses how the toy model of section 5.4 could be derived from a GUT model.

In the toy model of section 5.4, the singlets $N$ and $S$ do not separately couple to complete $S U(5)$ representations (see Eqn.(5.15)). If the extra fields introduced to solve the fine tuning problcm were originally part of $(5+\overline{5})$ multiplets, then

\footnotetext{
${ }^{10} \mathrm{It}$ is assumed that the model contains some mechanism to generate $-m_{N}^{2} \sim(100 \mathrm{GeV})^{2}-$ $(200 \mathrm{GeV})^{2}$; for example, the singlet is coupled to an extra $(5+\overline{5})$.
} 

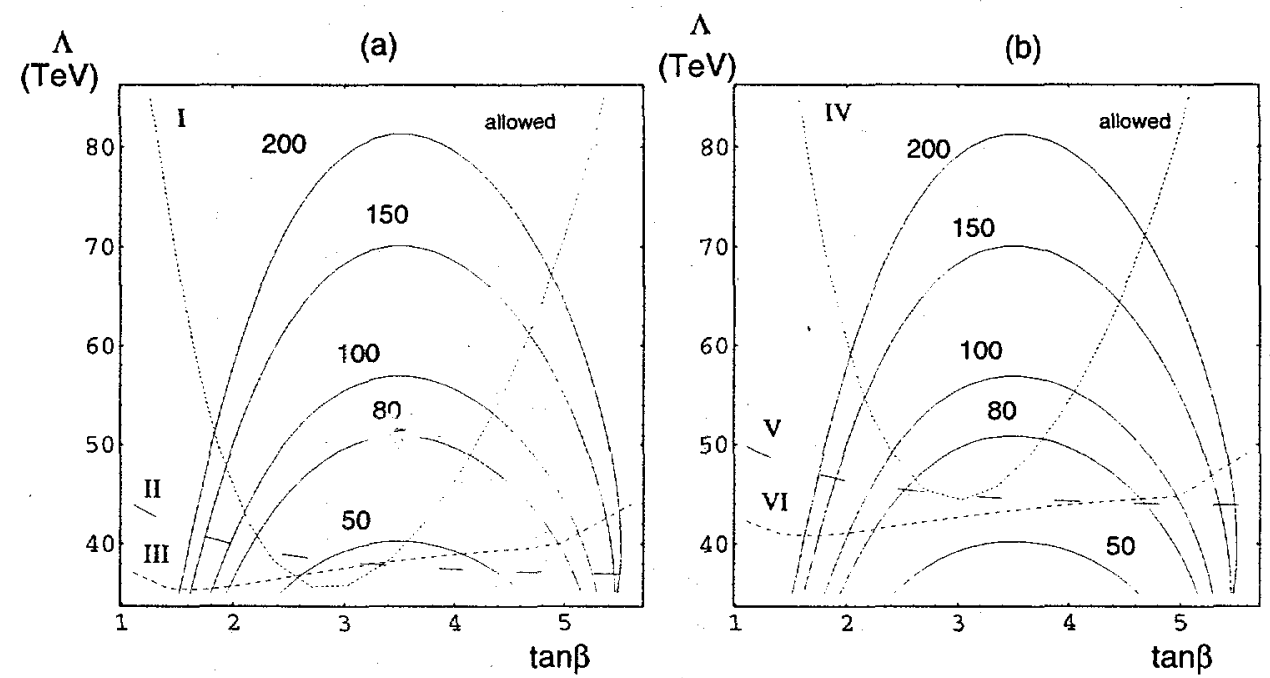

Figure 4

Figure 5.4: Contours of $c\left(m_{Z}^{2} ; \lambda_{H}\right)=(50,80,100,150,200)$ for the NMSSM of Section 5 with $\lambda_{H}=0.1$ and a messenger particle content of one $(5+\overline{5})$. The constraints considered are: (I) $m_{h}+m_{a}=m_{Z}$, (II) $m_{\tilde{e}_{R}}=75 \mathrm{GeV}$, (III) $m_{\tilde{\chi}_{1}^{0}}+$ $m_{\tilde{\chi}_{2}^{0}}=160 \mathrm{GeV}$, (IV) $m_{h}=92 \mathrm{GeV}$, (V) $m_{\tilde{e}_{R}}=85 \mathrm{GeV}$, and (VI) $m_{\tilde{\chi}_{1}^{0}}+m_{\tilde{\chi}_{2}^{0}}=$ $180 \mathrm{GeV}$. A central value of $m_{t}=175 \mathrm{GeV}$ is assumed. 
the missing triplets (missing doublets) necessarily couple to the singlet $S(N)$. The triplets must be heavy in order to suppress their contribution to the soft SUSY breaking mass parameters. If the only other mass scale is $M_{G U T}$, then they must acquire a mass at $M_{G U T}$. This is just the usual problem of splitting a $(5+\overline{5})$ [13]. For example, if the superpotential in the messenger sector contains four $(5+\overline{5})$ 's,

$$
W=\lambda_{1} S \overline{5}_{l 1} 5_{l 1}+\lambda_{2} S \overline{5}_{l 2} 5_{l 2}+\lambda_{3} S \overline{5}_{l 3} 5_{l 3}+\lambda_{4} S \overline{5}_{q} \overline{5}_{q}
$$

then the $S U(3)$ triplets in the $\left(\overline{5}_{l}+5_{l}\right)$ 's and the $S U(2)$ doublet in $\left(\overline{5}_{q}+5_{q}\right)$ must be heavy at $M_{G U T}$ so that in the low energy theory there are three doublets and one triplet coupling to $S$. This problem can be solved using the method of Barbieri, Dvali and Strumia [73] that solves the usual Higgs doublet-triplet splitting problem. The mechanism in this model is attractive since it is possible to make either the doublets or triplets of a quintet heavy at the GUT scale. Their model is now briefly described.

The gauge group is $S U(5) \times S U(5)^{\prime}$, with the particle content $\Sigma(24,1)$, $\Sigma^{\prime}(1,24), \Phi(5, \overline{\overline{5}})$ and $\bar{\Phi}(\overline{5}, 5)$ and the superpotential can be written as

$$
\begin{aligned}
W= & \bar{\Phi}_{\alpha^{\prime}}^{\beta}\left(M_{\Phi} \delta_{\beta^{\prime}}^{\alpha^{\prime}} \delta_{\beta}^{\alpha}+\lambda \Sigma_{\beta}^{\alpha} \delta_{\beta^{\prime}}^{\alpha^{\prime}}+\lambda^{\prime} \Sigma_{\beta^{\prime}}^{\alpha^{\prime}} \delta_{\beta}^{\alpha}\right) \Phi_{\alpha}^{\beta^{\prime}}+ \\
& +\frac{1}{2} M_{\Sigma} \operatorname{Tr}\left(\Sigma^{2}\right)+\frac{1}{2} M_{\Sigma^{\prime}} \operatorname{Tr}\left(\Sigma^{\prime 2}\right)+ \\
& \frac{1}{3} \lambda_{\Sigma} \operatorname{Tr} \Sigma^{3}+\frac{1}{3} \lambda_{\Sigma^{\prime}} \operatorname{Tr} \Sigma^{\prime 3}
\end{aligned}
$$

A supersymmetric minimum of the scalar potential satisfies the $F$ - flatness conditions

$$
0=F_{\bar{\Phi}}=\left(M_{\Phi} \delta_{\beta^{\prime}}^{\alpha^{\prime}} \delta_{\beta}^{\alpha}+\lambda \Sigma_{\beta}^{\alpha} \delta_{\beta^{\prime}}^{\alpha^{\prime}}+\lambda^{\prime} \Sigma_{\beta^{\prime}}^{\alpha^{\prime}} \delta_{\beta}^{\alpha}\right) \Phi_{\alpha}^{\beta^{\prime}}
$$




$$
\begin{aligned}
& 0=F_{\Sigma}=\frac{1}{2} M_{\Sigma} \Sigma_{\alpha}^{\beta}+\frac{1}{2}\left(\lambda \bar{\Phi}_{\alpha^{\prime}}^{\beta} \Phi_{\alpha}^{\alpha^{\prime}}-\lambda \frac{1}{5} \delta_{\alpha}^{\beta} \operatorname{Tr}(\bar{\Phi} \Phi)\right)+\lambda_{\Sigma}\left(\Sigma^{2}-\frac{1}{5} \operatorname{Tr} \Sigma^{2}\right) \\
& 0=F_{\Sigma^{\prime}}=\frac{1}{2} M_{\Sigma^{\prime}} \Sigma_{\alpha^{\prime}}^{\prime \beta^{\prime}}+\frac{1}{2}\left(\lambda^{\prime} \bar{\Phi}_{\alpha^{\prime}}^{\alpha} \Phi_{\alpha}^{\beta^{\prime}}-\lambda^{\prime} \frac{1}{5} \delta_{\alpha^{\prime}}^{\beta^{\prime}} \operatorname{Tr}(\bar{\Phi} \Phi)\right)+\lambda_{\Sigma^{\prime}}\left(\Sigma^{\prime 2}-\frac{1}{5} \operatorname{Tr} \Sigma^{\prime 2}\right) .
\end{aligned}
$$

With the ansatz ${ }^{11}$

$$
\Sigma=v_{\Sigma} \operatorname{diag}(2,2,2,-3,-3), \Sigma^{\prime}=v_{\Sigma^{\prime}} \operatorname{diag}(2,2,2,-3,-3)
$$

the $F_{\bar{\Phi}}=0$ condition is

$$
\operatorname{diag}\left[M_{3}, M_{3}, M_{3}, M_{2}, M_{2}\right] \cdot \operatorname{diag}\left[v_{3}, v_{3}, v_{3}, v_{2}, v_{2}\right]=0
$$

where $M_{3}=M_{\Phi}+2 \lambda v_{\Sigma}+2 \lambda^{\prime} v_{\Sigma^{\prime}}$ and $M_{2}=M_{\Phi}-3 \lambda v_{\Sigma}-3 \lambda^{\prime} v_{\Sigma^{\prime}}$ and the second matrix is the vev of $\Phi$. To satisfy this condition, there is a discrete choice for the pattern of vev of $\Phi$ : i) $v_{3} \neq 0$ and $M_{3}=0$ or ii) $v_{2} \neq 0$ and $M_{2}=0$. Substituting either i) or ii) in the $F_{\Sigma}$ and $F_{\Sigma^{\prime}}$ conditions then determines $v_{3}$ (or $v_{2}$ ). With two sets of fields, $\Phi_{1}, \bar{\Phi}_{1}$ with $v_{3} \neq 0$ and $\Phi_{2}, \bar{\Phi}_{2}$ with $v_{2} \neq 0$, we have the following pattern of symmetry breaking

$$
\begin{array}{cl}
S U(5) \times S U(5)^{\prime} \stackrel{v_{\Sigma}, v_{\Sigma^{\prime}}}{\rightarrow} & (S U(3) \times S U(2) \times U(1)) \times(S U(3) \times S U(2) \times U(1))^{\prime} \\
& \stackrel{v_{3}, v_{2}}{\rightarrow} S M(\text { the diagonal subgroup) } .
\end{array}
$$

If the scales of the two stages of symmetry breaking are about equal, i.e. $v_{\Sigma}, v_{\Sigma^{\prime}}, \sim$ $v_{3}, v_{2} \sim M_{G U T}$, then the SM gauge couplings unify at the scale $M_{G U T}$.

\footnotetext{
${ }^{11}$ The two possible solutions to the $F$-flatness conditions are $\Sigma=v_{\Sigma} \operatorname{diag}(2,2,2,-3,-3)$ and $\Sigma=v_{\Sigma} \operatorname{diag}(1,1,1,1,-4)$.

${ }^{12}$ See [73] and [81] for models which give this structure of vevs for the $\Phi$ fields without using the adjoints.
} 
The particular structure of the vevs of $\Phi_{1}$ and $\Phi_{2}$ can be used to split representations as follows.

Consider the Higgs doublet-triplet splitting problem. With the particle content $5_{h}(5,1), \overline{5}_{h}(\overline{5}, 1)$ and $X(1,5), \bar{X}(1, \overline{5})$ and the superpotential

$$
W=5_{h \alpha} \bar{X}^{\alpha^{\prime}} \bar{\Phi}_{1 \alpha^{\prime}}^{\alpha}+\overline{5}_{h}^{\alpha} X_{\alpha^{\prime}} \Phi_{1 \alpha}^{\alpha^{\prime}}
$$

the $S U(3)$ triplets in $5_{h}, \overline{5}_{h}$ and $X, \bar{X}$ acquire a mass of order $M_{G U T}$ whereas the doublets in $5_{h}, \overline{5}_{h}$ and $X, \bar{X}$ are massless. Now only one pair of doublets is wanted in the low energy theory (in addition to the usual matter fields). The doublets in $X, \bar{X}$ can be made heavy by a bare mass term $M_{G U T} X \bar{X}$. Then the doublets in $5_{h}, \overline{5}_{h}$ are the standard Higgs doublets. But if all terms consistent with symmetries are allowed in the superpotential, then allowing $M_{G U T} \Phi_{1} \bar{\Phi}_{1}, M_{G U T} X \bar{X}, 5_{h} \bar{X} \Phi_{1}$ and $\overline{5}_{h} X \bar{\Phi}_{1}$ implies that a bare mass term for $5_{h} \overline{5}_{h}$ is allowed. Of course, a $\mu$ term $\mu 5_{h} \overline{5}_{h}$ of the order of the weak scale can be put in by hand, as in section 5.4. However, it is theoretically more desirable to relate all electroweak mass scales to the original SUSY breaking scale. So, the $\mu$ term should be related to the size of SUSY breaking. Recall that in section 5.5 it was shown that the NMSSM is phenomenologically viable and "unfine tuned" in these models.

The vev structure of $\Phi_{2}, \bar{\Phi}_{2}$ can be used to make the doublets in a $5+\overline{5}$ heavy. Again, there are two pairs of light triplets and one of these pairs can be given a mass at the GUT scale.

This mechanism of making either doublets or triplets in a $(5+\overline{5})$ heavy can be 
used to show how the model of section 5.4 is derivable from a GUT. The model with three messenger doublets and one triplet is obtained from a GUT with the following superpotential

$$
\begin{aligned}
W= & S 5 \overline{5}+S 5_{l} \overline{5}_{l}+S X_{l} \bar{X}_{l}+ \\
& 5_{l} \bar{X}_{l} \bar{\Phi}_{1}+\overline{5}_{l} X_{l} \Phi_{1}+ \\
& 5_{q} \bar{X}_{q} \bar{\Phi}_{2}+\overline{5}_{q} X_{q} \Phi_{2}+ \\
& M_{G U T} X_{h} \bar{X}_{h}+5_{h} \bar{X}_{h} \bar{\Phi}_{1}+\overline{5}_{h} X_{h} \Phi_{1}+\mu 5_{h} \overline{5}_{h} \\
& +N^{3}+N 5_{q} \overline{5}_{q}+N X_{q} \bar{X}_{q} .
\end{aligned}
$$

Here, some of the "extra" triplets and doublets resulting from splitting $(5+\overline{5})$ 's are massless at the GUT scale. For example, the "extra" light doublets are used as the additional messenger leptons. After inserting the vevs and integrating out the heavy states, this corresponds to the superpotential in Eqn.(5.15) with the transcription:

$$
\begin{aligned}
5, \overline{5} & \rightarrow q_{1}, \bar{q}_{1}+l_{1}, \bar{l}_{1} \\
5_{l}, \overline{5}_{l} & \rightarrow l_{2}, \bar{l}_{2} \\
X_{l}, \bar{X}_{l} & \rightarrow l_{3}, \bar{l}_{3} \\
5_{q}, \overline{5}_{q} & \rightarrow q_{2}, \bar{q}_{2} \\
X_{q}, \bar{X}_{q} & \rightarrow q_{3}, \bar{q}_{3} .
\end{aligned}
$$

This section is concluded with a remark about light singlets in SUSY-GUT's 
with low energy gauge mediated SUSY breaking. ${ }^{13}$ In a SUSY-GUT with a singlet $N$ coupled to the Higgs multiplets, there is a potential problem of destabilizing the $m_{\text {weak }} / M_{G U T}$ hierarchy, if the singlet is light and if the Higgs triplets have a SUSY invariant mass of $O\left(M_{G U T}\right)$ [85]. In the LEGM models, a B-type mass for the Higgs triplets and doublets is generated at one-loop with gauginos and Higgsinos in the loop, and with SUSY breaking coming from the gaugino mass. Since SUSY breaking (the gaugino mass and the soft scalar masses) becomes soft above the messenger scale, $\Lambda_{\text {mess }} \sim 100 \mathrm{TeV}$, the B-type mass term generated for the Higgs triplets is suppressed, i.e., it is $O\left((\alpha / 4 \pi) M_{2} \Lambda_{m e s s}^{2} / M_{G U T}\right)$. Similarly the soft mass squareds for the Higgs triplets are $O\left(m_{\text {weak }}^{2} \Lambda_{m e s s}^{2} / M_{G U T}^{2}\right)$. Since the triplets couple to the singlet $N$, the soft scalar mass and $B$-term generates at one-loop a linear term for the scalar and $F$-component of $N$ respectively. These tadpoles are harmless since the SUSY breaking masses for the triplets are so small. This is to be contrasted with supergravity theories, where the $B$-term $O\left(m_{\text {weak }} M_{G U T}\right)$ and the soft mass $\sim O\left(m_{\text {weak }}\right)$ for the triplet Higgs generates a mass for the Higgs doublet that is at least $\sim O\left(\sqrt{m_{\text {weak }} M_{G U T}} /(4 \pi)\right)$.

\subsection{One complete Model}

The model is based on the gauge group $G_{l o c}=S U(5) \times S U(5)^{\prime}$ and the global symmetry group $G_{g l o}=Z_{3} \times Z_{3}^{\prime} \times Z_{4}$. The global symmetry acts universally on the three generations of the SM. The particle content and their $G_{l o c} \times G_{g l o}$

\footnotetext{
${ }^{13}$ The author thanks H. Murayama for bringing this to his attention.
} 
quantum numbers are given in Tables 5.2 and 5.3. The most general renormalizable superpotential that is consistent with these symmetries is

$$
W=W_{1}+W_{2}+W_{3}+W_{4}+W_{5}+W_{6}+W_{7}
$$

where,

$$
\begin{aligned}
W_{1}= & \frac{1}{2} M_{\Sigma} \operatorname{Tr} \Sigma^{2}+\frac{1}{3} \lambda_{\Sigma} \operatorname{Tr} \Sigma^{3}+\frac{1}{2} M_{\Sigma^{\prime}} \operatorname{Tr} \Sigma^{\prime 2}+\frac{1}{3} \lambda_{\Sigma^{\prime}} \operatorname{Tr} \Sigma^{\prime 3} \\
& +\Phi_{2}\left(M_{\Phi_{2}}+\lambda_{\Phi_{2}} \Sigma+\lambda_{\Phi_{2}}^{\prime} \Sigma^{\prime}\right) \bar{\Phi}_{2} \\
& +\Phi_{1}\left(M_{\Phi_{1}}+\lambda_{\Phi_{1}} \Sigma+\lambda_{\Phi_{1}}^{\prime} \Sigma^{\prime}\right) \bar{\Phi}_{1}, \\
W_{2}= & M_{1} \bar{X}_{l} X \\
W_{3}= & \lambda_{1} \overline{5}_{h} \Phi_{1} X_{h}+\bar{\lambda}_{1} 5_{h} \bar{\Phi}_{1} \bar{X}_{h}+\lambda_{2} \overline{5}_{l} \Phi_{1} X_{l}+\bar{\lambda}_{2} 5_{l} \bar{\Phi}_{1} \bar{X}_{l}, \\
W_{4}= & \lambda_{3} \overline{5}_{q} \Phi_{2} X_{q}+\bar{\lambda}_{3} 5_{q} \bar{\Phi}_{2} \bar{X}_{q}, \\
W_{5}= & \lambda_{6} S 5_{l} \overline{5}_{l}+\lambda_{7} S 5_{q} \overline{5}_{q}+\lambda_{8} S \bar{X}_{h} X_{l}+\lambda_{9} S \bar{X} X_{h}+\frac{1}{3} \lambda_{S} S^{3}, \\
W_{6}= & -\lambda_{H} 5_{h} \overline{5}_{h} N+\frac{1}{3} \lambda_{N} N^{3}+\bar{\lambda}_{q} N X \bar{X} \\
& +\lambda_{10} N^{\prime} \bar{X} X_{q}+\lambda_{11} N^{\prime} \bar{X}_{q} X+\frac{1}{3} \lambda_{N^{\prime}} N^{\prime 3}, \\
W_{7}= & \lambda_{i j}^{D} \overline{5}_{i} 10_{j} \overline{5}_{h}+\lambda_{i j}^{U} 10_{i} 10_{j} 5_{h} . \\
&
\end{aligned}
$$

The origin of each of the $W_{i}$ 's appearing in the superpotential is easy to understand. In computing the $F=0$ equations at the GUT scale, the only non-trivial contributions come from fields appearing in $W_{1}$, since all other $W_{i}$ s are bilinear in fields that do not acquire vevs at the GUT scale. The function of $W_{1}$ is to generate the vevs $\Sigma, \Sigma^{\prime} \sim \operatorname{diag}[2,2,2,-3,-3], \bar{\Phi}_{2}^{T}=\Phi_{2} \sim \operatorname{diag}[0,0,0,1,1]$ and $\bar{\Phi}_{1}^{T}=\Phi_{1} \sim$ $\operatorname{diag}[1,1,1,0,0]$. These vevs are necessary to break $G_{l o c} \rightarrow S U(3)_{c} \times S U(2) \times U(1)_{Y}$ 
(this was explained in section 5.6). The role of $W_{3}$ and $W_{4}$ is to generate the necessary splitting within the many $(5+\overline{5})$ 's of $G_{l o c}$ that is necessary to solve the usual doublet-triplet splitting problem, as well as to solve the fine tuning problem that is discussed in sections 5.3, 5.4 and 5.5. The messenger sector is given by $W_{5}$. It will shortly be demonstrated that at low energies this sector contains three vector-like doublets and one vector-like triplet. The couplings in $W_{6}$ and $W_{7}$ at low energies contain the electroweak symmetry breaking sector of the NMSSM, the Yukawa couplings of the SM fields, and the two light vector-like triplets necessary to maintain the few percent prediction for $\sin ^{2} \theta_{W}$ as well as to generate a vev for $N$.

Next it is shown that the low energy theory of this model is the model that is discussed in section 5.5 .

Inserting the vevs for $\Phi_{1}$ and $\bar{\Phi}_{1}$ into $W_{3}$, the following mass matrix for the colored triplet chiral multiplets is obtained:

$$
\left(\overline{5}_{h}, \bar{X}_{h}, \overline{5}_{l}, \bar{X}_{l}\right)\left(\begin{array}{ccccc}
0 & \lambda_{1} v_{\Phi_{1}} & 0 & 0 & 0 \\
\bar{\lambda}_{1} v_{\Phi_{1}} & 0 & 0 & 0 & 0 \\
0 & 0 & 0 & \lambda_{2} v_{\Phi_{1}} & 0 \\
0 & 0 & \bar{\lambda}_{2} v_{\Phi_{1}} & 0 & M_{1}
\end{array}\right)\left(\begin{array}{c}
5_{h} \\
X_{h} \\
5_{l} \\
X_{l} \\
X
\end{array}\right)
$$

and all other masses are zero. There are a total of four vector-like colored triplet fields that are massive at $M_{G U T}$. These are the triplet components of $\left(5_{h}, \bar{X}_{h}\right)$, $\left(\overline{5}_{h}, X_{h}\right),\left(\overline{5}_{l}, X_{l}\right)$ and $\left(\bar{X}_{l}, T_{H}\right)$, where $T_{H}$ is that linear combination of triplets in 
$5_{l}$ and $X$ that marries the triplet component of $\bar{X}_{l}$. The orthogonal combination to $T_{H}, T_{L}$, is massless at this scale. The massless triplets at $M_{G U T}$ are $\left(5_{q}, \overline{5}_{q}\right)$, $\left(X_{q}, \bar{X}_{q}\right)$ and $\left(\bar{X}, T_{L}\right)$, for a total of three vector-like triplets. By inspection, the only light triplets that couple to $S$ at a renormalizable level are $5_{q}$ and $\overline{5}_{q}$, which was desirable in order to solve the fine tuning problem. Further, since $X$ contains a component of $T_{L}$, the couplings of the other light triplets to the singlets $N$ and $N^{\prime}$ are

$$
W_{e f f}=\lambda_{10} N^{\prime} \bar{X} X_{q}+\bar{\lambda}_{11} N^{\prime} \bar{X}_{q} T_{L}+\lambda_{q} N T_{L} \bar{X}
$$

where $\lambda_{q}=\bar{\lambda}_{q} \cos \alpha^{\prime}, \bar{\lambda}_{11}=\lambda_{11} \cos \alpha^{\prime}$ and $\alpha^{\prime}$ is the mixing angle between the triplets in $5_{l}$ and $X$, i.e., $T_{L}=\cos \alpha^{\prime} X-\sin \alpha^{\prime} 5_{l}$. The $\lambda_{q} N T_{L} \bar{X}$ coupling is also desirable to generate acceptable $\mu$ and $\mu_{3}^{2}$ terms (see section 5.5).

In section $5.4,5.5$ it was also demonstrated that with a total of three messenger doublets the fine tuning required in electroweak symmetry breaking could be alleviated. By inserting the vev for $\Phi_{2}$ into $W_{4}$, the doublet mass matrix is given as

$$
\left(\bar{X}_{l}, \overline{5}_{q}, \bar{X}_{q}\right)\left(\begin{array}{ccc}
M_{1} & 0 & 0 \\
0 & 0 & \lambda_{3} v_{\Phi_{2}} \\
0 & \bar{\lambda}_{3} v_{\Phi_{2}} & 0
\end{array}\right)\left(\begin{array}{c}
X \\
5_{q} \\
X_{q}
\end{array}\right) \text {. }
$$

and all other masses are zero. At $M_{G U T}$ the heavy doublets are $\left(\bar{X}_{l}, X\right),\left(5_{q}, \bar{X}_{q}\right)$ and $\left(\overline{5}_{q}, X_{q}\right)$, leaving the four vector-like doublets in $\left(5_{h}, \overline{5}_{h}\right),\left(5_{l}, \overline{5}_{l}\right),\left(\bar{X}, X_{l}\right)$ and $\left(X_{h}, \bar{X}_{h}\right)$ massless at this scale. Of these four pairs, $\left(5_{h}, \overline{5}_{h}\right)$ are the usual Higgs doublets and the other three pairs couple to $S$. 
The (renormalizable) superpotential at scales below $M_{G U T}$ is then

$$
\begin{aligned}
W= & \lambda_{q} N \bar{q}_{2} q_{2}+\frac{1}{3} \lambda_{N} N^{3}+\lambda_{10} N^{\prime} q_{3} \bar{q}_{2} \\
& +\lambda_{11} N^{\prime} q_{2} \bar{q}_{3}-\lambda_{H} N H_{u} H_{d}+\frac{1}{3} \lambda_{N^{\prime}} N^{3} \\
& +\lambda_{6} S \bar{l}_{1} l_{1}+\lambda_{7} S \bar{q}_{1} q_{1}+\lambda_{8} S \bar{l}_{2} l_{2} \\
& +\lambda_{9} S \bar{l}_{3} l_{3}+\frac{1}{3} \lambda_{S} S^{3}+W_{7}
\end{aligned}
$$

where the fields have been relabeled to make, in an obvious notation, their $S U(3) \times$ $S U(2) \times U(1)$ quantum numbers apparent.

This section concludes with comments about the choice of $Z_{4}$ as a discrete symmetry and about non-renormalizable operators in our model.

The usual $R$-parity violating operators $10_{S M} \overline{5}_{S M} \overline{5}_{S M}$ are not allowed by the discrete symmetries, even at the non-renormalizable level. In fact, $R$-parity is a good symmetry of the effective theory below $M_{G U T}$. By inspection, the fields that acquire vevs at $M_{G U T}$ are either invariant under $Z_{4}$ or have a $Z_{4}$ charge of 2 (for example, $\left.\Phi_{1}\right)$, so that a $Z_{2}$ symmetry is left unbroken. In fact, the vevs of the other fields $S, N, N^{\prime}$ and the Higgs doublets do not break this $Z_{2}$ either. By inspecting the $Z_{4}$ charges of the SM fields, we see that the unbroken $Z_{2}$ is none other than the usual $R$-parity. So at $M_{G U T}$, the discrete symmetry $Z_{4}$ is broken to $R_{p}$. Also note that the $Z_{4}$ symmetry is sufficient to maintain, to all orders in $1 / M_{P l}$ operators, the vev structure of $\Phi_{1}$ and $\Phi_{2}$, i.e., to forbid unwanted couplings between $\Phi_{1}$ and $\Phi_{2}$ that might destabilize the vev structure [81]. This pattern of vevs was essential to solve the doublet-triplet splitting problem. It is interesting that both $R$-parity 
invariance and requiring a viable solution to the doublet-triplet splitting problem can be accommodated by the same $Z_{4}$ symmetry.

The non-SM matter fields (namely, the messenger 5's and $X$ 's and the light triplets ) have the opposite charge to the SM matter fields under the unbroken $Z_{2}$. Thus, there is no mass mixing between the SM and the non-SM matter fields.

Dangerous proton decay operators are forbidden in this model by the discrete symmetries. Some higher dimension operators that lead to proton decay are allowed, but are sufficiently suppressed. These are discussed below.

Renormalizable operators such as $10_{S M} 10_{S M} 5_{q}$ and $10_{S M} \overline{5}_{S M} \overline{5}_{q}$ are forbidden by the $Z_{3}$ symmetries. This is necessary to avoid a large proton decay rate. A dimension-6 proton decay operator is obtained by integrating out the colored triplet scalar components of $5_{q}$ or $\overline{5}_{q}$. Since the colored scalars in $5_{q}$ and $\overline{5}_{q}$ have a mass $\sim O(50 \mathrm{TeV})$, the presence of these operators would have led to an unacceptably large proton decay rate.

The operators $10_{S M} 10_{S M} 10_{S M} \overline{5}_{S M} / M_{P l}$ and $10_{S M} 10_{S M} 10_{S M} \overline{5}_{S M} \times$ $\left(\Phi \bar{\Phi} / M_{P l}^{2}\right)^{n} / M_{P l}$, which give dimension-5 proton decay operators, are also forbidden by the two $Z_{3}$ symmetries. The allowed non-renormalizable operators that generate dimension -5 proton decay operators are suffuciently suppressed. The operator $10_{S M} 10_{S M} 10_{S M} \overline{5}_{S M} N^{\prime} /\left(M_{P l}\right)^{2}$, for example, is allowed by the discrete symmetries, but the proton decay rate is safe since $v_{N^{\prime}} \sim 1 \mathrm{TeV}$.

The operators $10_{i} \overline{5}_{j} \bar{\Phi}_{1}\left(\bar{X}\right.$ or $\left.\bar{X}_{q}\right) / M_{P l}$ could, in principle, also lead to a large proton decay rate. Setting $\bar{\Phi}_{1}$ to its vev, the superpotential couplings, for example, 
$\lambda_{i j}\left(U_{i}^{c} D_{j}^{c} \bar{X}(\overline{3})+Q_{i} L_{j} \bar{X}(\overline{3})\right)$ are generated with $\lambda_{i j}$ suppressed only by $v_{\Phi_{1}} / M_{P l}$. In this model the colored triplet (scalar) components of $\bar{X}$ and $\bar{X}_{q}$ have a mass $m_{\tilde{q}} \sim$ $500 \mathrm{GeV}$, giving a potentially large proton decay rate. But, in this model these operators are forbidden by the discrete symmetries. The operator $10_{i} \overline{5}_{j} \bar{\Phi}_{1} \bar{X} S / M_{P l}^{2}$ is allowed giving a four SM fermion proton decay operator with coefficient $\sim$ $\left(v_{\Phi_{1}} v_{S} / M_{P l}^{2}\right)^{2} / m_{\tilde{q}}^{2} \sim 10^{-34} \mathrm{GeV}^{-2}$. This is smaller than the coefficient generated by exchange of the heavy gauge bosons of mass $M_{G U T}$, which is $\sim g_{G U T}^{2} / M_{G U T}^{2} \sim$ $1 / 210^{-32} \mathrm{GeV}^{-2}$ and so this operator leads to proton decay at a tolerable rate.

With our set of discrete symmetries, some of the messenger states and the light color triplets are stable at the renormalizable level. Non-renormalizable operators lead to decay lifetime for some of these particles of more than about 100 seconds. This is a problem from the viewpoint of cosmology, since these particles decay after Big-Bang Nucleosynthesis (BBN). With a non-universal choice of discrete symmetries, it might be possible to make these particles decay before BBN through either small renormalizable couplings to the third generation (so that the constraints from proton decay and FCNC are avoided) or non-renormalizable operators. Alternatively, if the reheat temperature is below the nass scale of these particles they will not have a relic abundance today. These issues, however, are beyond the scope of this chapter.

\subsection{Summary}

In this chapter the fine tuning required in models of low energy gauge-mediated 


\begin{tabular}{||l||l|l|l|l||}
\hline$\Psi$ & $\overline{5}_{i}$ & $10_{i}$ & $5_{h}$ & $\overline{5}_{h}$ \\
\hline$G_{l o c}$ & $(\overline{5}, 1)$ & $(10,1)$ & $(5,1)$ & $(\overline{5}, 1)$ \\
\hline$Z_{3}$ & 1 & $a$ & $a$ & $a^{2}$ \\
\hline$Z_{3}^{\prime}$ & $b$ & 1 & 1 & $b^{2}$ \\
\hline$Z_{4}$ & $c$ & $c$ & $c^{2}$ & $c^{2}$ \\
\hline
\end{tabular}

\begin{tabular}{||l||l|l|l|l|l|l||}
\hline$\Psi$ & $\Sigma$ & $\Sigma^{\prime}$ & $\bar{\Phi}_{2}$ & $\Phi_{2}$ & $\bar{\Phi}_{1}$ & $\Phi_{1}$ \\
\hline$G_{\text {loc }}$ & $(24,1)$ & $(1,24)$ & $(\overline{5}, 5)$ & $(5, \overline{5})$ & $(\overline{5}, 5)$ & $(5, \overline{5})$ \\
\hline$Z_{3}$ & 1 & 1 & 1 & 1 & 1 & 1 \\
\hline$Z_{3}^{\prime}$ & 1 & 1 & 1 & 1 & 1 & 1 \\
\hline$Z_{4}$ & 1 & 1 & 1 & 1 & $c^{2}$ & $c^{2}$ \\
\hline
\end{tabular}

\begin{tabular}{||l||l|l|l|l|l|l||}
\hline$\Psi$ & $5_{l}$ & $\overline{5}_{l}$ & $X_{l}$ & $\bar{X}_{l}$ & $5_{q}$ & $\overline{5}_{q}$ \\
\hline$G_{l o c}$ & $(5,1)$ & $(\overline{5}, 1)$ & $(1,5)$ & $(1, \overline{5})$ & $(5,1)$ & $(\overline{5}, 1)$ \\
\hline$Z_{3}$ & $a^{2}$ & 1 & 1 & $a$ & 1 & $a^{2}$ \\
\hline$Z_{3}^{\prime}$ & 1 & 1 & 1 & 1 & $b^{2}$ & $b$ \\
\hline$Z_{4}$ & $c^{2}$ & $c^{2}$ & 1 & 1 & 1 & 1 \\
\hline
\end{tabular}

Table 5.2: $S U(5) \times S U(5)^{\prime} \times Z_{3} \times Z_{3}^{\prime} \times Z_{4}$ quantum numbers for the fields of the model discussed in section 7 . The generators of $Z_{3} \times Z_{3}^{\prime} \times Z_{4}$ are labeled by $(a, b, c)$. The three SM generations are labeled by the index $i$. 


\begin{tabular}{||l||l|l|l|l|l|l||}
\hline$\Psi$ & $X_{q}$ & $\bar{X}_{q}$ & $X_{h}$ & $\bar{X}_{h}$ & $X$ & $\bar{X}$ \\
\hline$G_{l o c}$ & $(1,5)$ & $(1, \overline{5})$ & $(1,5)$ & $(1, \overline{5})$ & $(1,5)$ & $(1, \overline{5})$ \\
\hline$Z_{3}$ & $a$ & 1 & $a$ & $a^{2}$ & $a^{2}$ & $a$ \\
\hline$Z_{3}^{\prime}$ & $b^{2}$ & $b$ & $b$ & 1 & 1 & $b^{2}$ \\
\hline$Z_{4}$ & 1 & 1 & 1 & 1 & 1 & 1 \\
\hline
\end{tabular}

\begin{tabular}{||l||l|l|l|l|l||}
\hline$\Psi$ & $S$ & $N$ & $N^{\prime}$ & $\phi_{+}$ & $\phi_{-}$ \\
\hline$Z_{3}$ & $a$ & 1 & $a$ & $a$ & $a$ \\
\hline$Z_{3}^{\prime}$ & 1 & $b$ & $b^{2}$ & 1 & 1 \\
\hline$Z_{4}$ & 1 & 1 & 1 & 1 & 1 \\
\hline
\end{tabular}

Table 5.3: $S U(5) \times S U(5)^{\prime} \times Z_{3} \times Z_{3}^{\prime} \times Z_{4}$ quantum numbers for the fields of the model discussed in section 7 . The generators of $Z_{3} \times Z_{3}^{\prime} \times Z_{4}$ are labeled by $(a, b, c)$. The three SM generations are labeled by the index $i$. 
SUSY breaking to obtain the correct $Z$ mass was quantified. It was demonstrated that the minimal model requires a fine tuning of order $\sim 7 \%$ if LEP2 does not discover a right-handed slepton. It was discussed how models with more messenger doublets than triplets can improve the fine tuning. In particular, a model with a messenger field particle content of three $(l+\bar{l})$ 's and only one $(q+\bar{q})$ was tuned to $\sim 40 \%$. It was found that it was necessary to introduce an extra singlet to give mass to some color triplets (close to the weak scale) which are required to maintain gauge coupling unification. It was also discussed how the vev and $F$-component of this singlet could be used to generate the $\mu$ and $B \mu$ terms. It was found that for some region of the parameter space this model requires $\sim 25 \%$ tuning and that limits from LEP do not constrain the parameter space. This is in contrast to an NMSSM with extra vector-like quintets and with one $(5+\overline{5})$ messenger field, for which it was found that a fine tuning of $\sim 1 \%$ is required and that limits from LEP do significantly constrain the parameter space.

It was further discussed how the model with split messenger field representations could be the low energy theory of a $S U(5) \times S U(5)$ GUT. A mechanism similar to the one used to solve the usual Higgs doublet-triplet splitting problem was used to split the messenger field representations. All operators consistent with gauge and discrete symmetries were allowed. In this model $R$-parity is the unbroken subgroup of one of the discrete symmetry groups. Non-renormalizable operators involving non-SM fields lead to proton decay, but at a safe level. 


\section{Chapter 6}

\section{Non-decoupling of the First and Second}

\section{Generation Scalars}

The supersymmetric contributions to the Flavor Changing Neutral Current processes may be suppressed by decoupling the scalars of the first and second generations. It is known, however, that the heavy scalars drive the stop masses squared negative through the two-loop Renormalization Group evolution. This tension is studied in detail. Two new items are included in this analysis: the effect of the top quark Yukawa coupling and the QCD corrections to the supersymmetric contributions to $\Delta m_{K}$. Even with Cabibbo-like mixing between the squarks of the first two generations, these squarks must be heavier than $\sim 40 \mathrm{TeV}$ to suppress $\Delta m_{K}$. This implies, in the case of a high scale of supersymmetry breaking, that the boundary value of the stop mass has to be greater than $\sim 7 \mathrm{TeV}$ to keep the stop mass squared positive at the weak scale. Low-energy supersymmetry breaking at a scale that is of the same order as the mass of the heavy scalars is also considered. In this case the finite parts of the two-loop diagrams are computed to estimate the contribution of the heavy scalar masses to the stop mass squared. It is found that for Cabibbo-like mixing between the squarks, the stop mass at the boundary needs to be larger than $\sim 2 \mathrm{TeV}$. Thus, for both cases, the large boundary value of 
the stop masses leads to an unnatural amount of fine tuning to obtain the correct $Z$ mass.

\subsection{Introduction}

The origin of electroweak symmetry breaking (EWSB) and the subsequent gauge hierarchy problem are two large mysteries of the Standard Model (SM). Supersymmetry (SUSY) provides a promising solution to these problems, by both stabilizing the weak scale against radiative corrections[6], and by naturally breaking the electroweak symmetry through the quantum corrections of the superpartner of the top quark to the Higgs boson mass [86]. It is known, however, that generic weak scale values for the masses of the first two generation scalars give rates for many flavor violating processes that are in disagreement with the experimental observation. The measured value of $\Delta m_{K}$ and detection limits for $\mu \rightarrow e \gamma$, and $\mu \rightarrow 3 e$, for example, require that the first two generation scalars be degenerate to within a few tenths of a percent if their masses are at the weak scale [65, 68]. Constraints from $C P$ violation are generally even more severe. Understanding the origin of this degeneracy is the supersymmetric flavor problemi. Attempts to resolve this puzzle without introducing any fine tuning include: using approximate non-abelian or abelian symmetries[87]; communicating supersymmetry breaking to the visible sector by gauge interactions that do not distinguish between flavors [11]; squark-quark mass matrix alignment [88]; and raising the soft masses of the first two generation scalars to the tens of $\mathrm{TeV}$ range $[89,90,91,92,93,94,95,96]$. 
The phenomenological viability and naturalness of this last scenario is the subject of this chapter. To suppress flavor changing processes, the heavy scalars must have masses between a few $\mathrm{TeV}$ and a hundred $\mathrm{TeV}$. The actual value depends on the degree of degeneracy and mixing between the masses of the first two generation scalars. As discussed in Reference [97], the masses of the heavy scalars cannot be made arbitrarily large without breaking color and charge. This is because the heavy scalar masses contribute to the two-loop Renormalization Group Equation (RGE) for the soft masses of the light scalars, such that the stop soft mass squared become more negative in RG scaling to smaller energy scales. This negative contribution is large if the scale at which supersymmetry breaking is communicated to the visible sector is close to the Grand Unification scale[97]. With the first two generation soft scalar masses $\approx 10 \mathrm{TeV}$, the initial value of the soft masses for the light stops must be $\approx(\text { few } \mathrm{TeV})^{2}$ to cancel this negative contribution [97] to obtain the correct vacuum. This requires, however, an unnatural amount of fine tuning to correctly break the electroweak symmetry[63, 64].

In this chapter these issues are analyzed. Two new items not previously discussed within this context are included: the effect of the large top quark Yukawa coupling, $\lambda_{t}$, in the RG evolution, that drives the stop soft mass squared more negative; and QCD radiative corrections in the $\Delta m_{K}$ constraint [98]. This modifies the bound on the heavy scalar masses that is consistent with the measured value of $\Delta m_{K}$. This, in turn, affects the minimum value of the initial scalar masses that is required to keep the scalar masses positive at the weak scale. 
This severe constraint obtained for the initial stop masses assumes that supersymmetry breaking occurs at a high scale. This leaves open the possibility that requiring positivity of the scalar masses is not a strong constraint if the scale of supersymmetry breaking is not much larger than the mass scale of the heavy scalars. In this chapter this possibility is investigated by computing the finite parts of the same two-loop diagrams responsible for the negative contribution to the light scalar RG equation, and using these results as an estimate of the twoloop contribution in an actual model of low-energy supersymmetry breaking. It is found that in certain classes of models, requiring positivity of the soft masses may place strong necessary conditions that such models must satisfy in order to be phenomenologically viable.

This chapter is organized as follows. In section 6.2 an overview of the ingredients of the analysis is presented. Some philosophy and notation is discussed. Section 6.2.1 discusses the constraints on the masses and mixings of the first two generation scalars obtained from $\Delta m_{K}$ after including QCD corrections. It is found, in particular, that Cabibbo-like mixing among both the first two generation left-handed squarks and right-handed squarks requires them to be heavier than $40 \mathrm{TeV}$. Section 6.2.2 discusses the logic of the RG analysis, and some formulas are presented. This analysis is independent of the $\Delta m_{K}$ analysis. Sections 6.3 and 6.4 apply this machinery to the cases of low-energy and high-energy supersymmetry breaking, respectively. Section 6.3 deals with the case in which the scale at which SUSY breaking is communicated to the SM sparticles is close to the 
mass of the heavy scalars. The finite parts of the two-loop diagrams are used to ,estimate the negative contribution of the heavy scalars. For Cabibbo-like mixing among the left-handed and right-handed squarks of the first two generations the boundary value of the stop masses has to greater than $\sim 2 \mathrm{TeV}$ to keep the stop masses squared positive at the weak scale. This results in a fine tuning of naively $1 \%$ in electroweak symmetry breaking [63]. Also discussed are the cases where there is $O(1)$ mixing among only the right or left squarks of the first two generations, and requiring positivity of the slepton masses squared implies a constraint on the stop masses of $\sim 1 \mathrm{TeV}$ if gauge-mediated boundary conditions are used to relate the two masses. This is comparable to the direct constraint on the initial stop masses. Section 6.4 considers the case where the SUSY breaking masses for the SM sparticles are generated at a high scale $\left(\sim 10^{16} \mathrm{GeV}\right)$. In this case, the negative contribution of the heavy scalars is enhanced by a large logarithm. Various boundary conditions for the stop and Higgs masses are considered and it is found that for an order of 0.2 degeneracy between the first two generation squarks, the boundary value of the stop mass needs to be larger than $\sim 7 \mathrm{TeV}$. This gives a fine tuning of naively $0.02 \%[63]$. For $O(1)$ mixing between the left (right) squarks only, the minimum initial value of the stop is $\sim 4(2) \mathrm{TeV}$. In section 6.5 the scale of supersymmetry breaking is varied between $50 \mathrm{TeV}$ and $2 \times 10^{16} \mathrm{GeV}$. Uppers bounds on the amount of degeneracy required between the first two generation scalars, that is consistent with positivity of the light scalar masses, naturalness in electroweak symmetry breaking, and the measured value of $\Delta m_{K}$, are obtained. 
These results are summarized in Figures 6.12 and 6.13. The results of this chapter are summarized in section 6.6. Appendix B discusses the computation of the two-loop diagrams which give the negative contribution of the heavy scalars to the light scalar mass squareds.

\subsection{Overview.}

The chiral particle content of the Minimal Supersymmetric Standard Model (MSSM) contains 3 generations of $\overline{\mathbf{5}}+\mathbf{1 0}$ representations of $S U(5)$. The supersymmetry must be softly broken to not be excluded by experiment. Thus the theory must also be supplemented by some "bare" soft supersymmetry breaking parameters, as well as a physical cutoff, $M_{S U S Y}$. The "bare" soft supersymmetry breaking parameters are then the coefficients appearing in the Lagrangian, defined with a cutoff $M_{S U S Y}$. It will be assumed for simplicity that the bare soft masses, $\tilde{m}_{i, 0}^{2}$, the bare gaugino masses $M_{A, 0}$, and a bare trilinear term for the stops, $\lambda_{t} A_{t, 0}$, are all generated close to this scale. The MSSM is then a good effective theory at energies below the scale $M_{S U S Y}$, but above the mass of the heaviest superpartner.

The physical observables at low-energies will depend on these parameters. If an unnatural degree of cancellation is required between the bare parameters of the theory to produce a measured observable, the theory may be considered to be fine tuned. Of course, it is possible that a more fundamental theory may resolve in a natural manner the apparent fine tuning. Solutions to the gauge-hierarchy problem are well-known examples of this. The Higgs boson mass of the SM is fine 
tuned if the $\mathrm{SM}$ is valid at energies above a few $\mathrm{TeV}$. This fine tuning is removed if at energies close to the weak scale the SM is replaced by a more fundamental theory that is supersymmetric[6].

One quantification of the fine tuning of an observable $\mathcal{O}$ with respect to a bare parameter $\lambda_{0}$ is given by Barbieri-Giudice [63] to be

$$
\Delta\left(\mathcal{O} ; \lambda_{0}\right)=(\delta \mathcal{O} / \mathcal{O}) /\left(\delta \lambda_{0} / \lambda_{0}\right)=\frac{\lambda_{0}}{\mathcal{O}} \frac{\partial}{\partial \lambda_{0}} \mathcal{O}
$$

It is argued that this only measures the sensitivity of $\mathcal{O}$ to $\lambda_{0}$, and care should be taken when interpreting whether a large value of $\Delta$ necessarily implies that $\mathcal{O}$ is fine tuned [64]. It is not the intent of this chapter to quantify fine tuning; rather, an estimate of the fine tuning is sufficient and Eqn.(6.1) will be used. In this chapter the value of $\mathcal{O}$ is considered extremely unnatural if $\Delta\left(\mathcal{O} ; \lambda_{0}\right)>100$.

The theoretical prediction for $\Delta m_{K}$ (within the MSSM) and its measured value are an example of such a fine tuning: Why should the masses of the first two generation scalars be degenerate to within $1 \mathrm{GeV}$, when their masses are $O(500 \mathrm{GeV})$ ? Phrased differently, the first two generation scalars must be extremely degenerate for the MSSM to not be excluded by the measured value of $\Delta m_{K}$. An important direction in supersymmetry model building is aimed at attempting to explain the origin of this degeneracy.

One proposed solution to avoid this fine tuning is to decouple the first two generation scalars since their masses are the most stringently constrained by the flavor violating processes $[89,90,91,92,94,95,96]$. In this scenario, some of the 
first two generation scalars have masses $M_{S} \gg m_{Z}$. To introduce some notation, $n_{5}\left(n_{10}\right)$ will denote the number of $\overline{\mathbf{5}}(\mathbf{1 0})$ scalars of the MSSM particle content that are very heavy ${ }^{1}$. Thus at energy scales $E \ll M_{S}$ the particle content is that of the MSSM, minus the $n_{5} \overline{\mathbf{5}}$ and $n_{10} \mathbf{1 0}$ scalars. In the literature this is often referred to as 'The More Minimal Supersymmetric Standard Model'[92].

There are, however, other possible and equally valid sources of fine tunings. The measured value of the $Z$ mass is such an example [63]. The minimum of the renormalized Higgs potential determines the value of the $Z$ mass which is already known from experiment. The vacuum expectation value (vev) of the Higgs field is, in turn, a function of the bare parameters of the theory. The relation used here, valid at the tree-level, is

$$
\frac{1}{2} m_{Z}^{2}=-\mu^{2}+\frac{m_{H_{d}}^{2}\left(\mu_{G}\right)-m_{H_{u}}^{2}\left(\mu_{G}\right) \tan ^{2} \beta}{\tan ^{2} \beta-1} .
$$

It is clear from this Equation that requiring correct electroweak symmetry breaking relates the value of the soft Higgs masses at the weak scale, $m_{H_{d}}^{2}\left(\mu_{G}\right)$ and $m_{H_{u}}^{2}\left(\mu_{G}\right)$, to the supersymmetric Higgs mass $\mu$. A numerical computation determines the dependence of $m_{H_{u}}^{2}\left(\mu_{G}\right)$ and $m_{H_{d}}^{2}\left(\mu_{G}\right)$ on the bare parameters $M_{A, 0}, m_{\tilde{t}_{i}, 0}^{2}$ and $M_{S}$. In the MSSM, the cancellation required between the bare parameters of the theory for it not to be excluded by the $Z$ mass increases as the scale of supersymmetry breaking is increased. Typically, the bare mass of the gluino, stops, and the first two generation squarks must be less than a few $\mathrm{TeV}$ and ten $\mathrm{TeV}$, respectively,

\footnotetext{
${ }^{1}$ It is assumed that the heavy scalars form complete $S U(5)$ multiplets to avoid a large FayetIlliopoulus $D$ - term at the one-loop level[96, 92].
} 
for successful electroweak symmetry breaking not to be fine tuned at more than the one per cent level $[63,64,96]$.

These two potential fine tuning problemsm - the supersymmetric flavor problem and that of electroweak symmetry breaking - are not completely independent, for they both relate to the size of supersymmetry breaking $[96,97]$. Thus the consistency of any theoretical framework that attempts to resolve one fine tuning issue can be tested by requiring that it not reintroduce any comparable fine tunings in other sectors of the theory. This is the situation for the case under consideration here. Raising the masses of the first two generation scalars can resolve the supersymmetric flavor problem. As discussed in [96], this results in a fine tuning of $m_{Z}^{2}$ through the two-loop dependence of $m_{H_{u}}^{2}\left(\mu_{G}\right)$ on $M_{S}$. There is, however, another source of fine tuning of $m_{Z}$ due to the heavy scalars: these large masses require that the bare masses of the stops, in particular, be typically larger than a few $\mathrm{TeV}$ to keep the soft masses squared positive at the weak scale [97]. This large value for the bare stop mass prefers a large value for the vev of the Higgs field, thus introducing a fine tuning in the electroweak sector. Further, this fine tuning is typically not less than the original fine tuning in the flavor sector. This is the central issue of this chapter.

\subsection{1 $\Delta m_{K}$ Constraints}

At the one-loop level the exchange of gluinos and squarks generates a $\Delta S=$ 2 operator. In the limit of interest here, $M_{3} \ll M_{S}$, the $\Delta S=2$ effective 
Lagrangian at the scale $M_{S}$ obtained by integrating out the squarks is

$$
\mathcal{L}_{\text {eff }}=\frac{\alpha_{S}^{2}\left(M_{S}\right)}{216 M_{S}^{2}}\left(C_{1} \mathcal{O}_{1}+\tilde{C}_{1} \tilde{\mathcal{O}}_{1}+C_{4} \mathcal{O}_{4}+C_{5} \mathcal{O}_{5}+\text { h.c. }\right)
$$

Terms that are $O\left(M_{3}^{2} / M_{S}^{2}\right)$ are subdominant and neglected. Next, the exact result is expanded in powers of $\delta_{L L, R R}=s_{L, R} c_{L, R} \eta_{L, R}\left(\tilde{m}_{1}^{2}-\tilde{m}_{2}^{2}\right)_{L L, R R} / \tilde{m}_{A V, L, R}^{2}$, where $\tilde{m}_{A V}^{2}$ is the average mass of the scalars, and where $\eta_{L, R}$ is the phase and $s_{L, R}$ is the 1-2 element of the $W_{L, R}$ matrix that appears at the gluino-squark-quark vertex ${ }^{2}$. Since this approximation underestimates the magnitude of the exact result this analysis is conservative[97]. The coefficients $C_{i}$ to leading order in $\delta_{L L}, \delta_{R R}$, are

$$
\begin{aligned}
& C_{1}=-22 \delta_{L L}^{d} \\
& C_{4}=24 \delta_{L L}^{d} \delta_{R R}^{d} \\
& C_{5}=-40 \delta_{L L}^{d} \delta_{R R}^{d} .
\end{aligned}
$$

The coefficient $\tilde{C}_{1}$ is obtained from $C_{1}$ with the replacement $\delta_{L L}^{d} \rightarrow \delta_{R R}^{d}$. The operators $\mathcal{O}_{i}$ are

$$
\begin{aligned}
\mathcal{O}_{1} & =\bar{d}_{L}^{a} \gamma_{\mu} s_{L, a} \bar{d}_{L}^{b} \gamma^{\mu} s_{L, b} \\
\mathcal{O}_{4} & =\bar{d}_{R}^{a} s_{L, a} \bar{d}_{L}^{b} s_{R, b} \\
\mathcal{O}_{5} & =\bar{d}_{R}^{a} s_{L, b} \bar{d}_{L}^{b} s_{R, a}
\end{aligned}
$$

and $\tilde{\mathcal{O}}_{1}$ is obtained from $\mathcal{O}_{1}$ with the replacement $L \rightarrow R$. The Wilson coefficients, $C_{1}-C_{5}$, are RG scaled from the scale of the squarks, $M_{S}$, to $900 \mathrm{MeV}$ using the

\footnotetext{
${ }^{2}$ In this chapter only 1-2 generation mixing is considered. Direct $L-R$ mass mixing is also neglected.
} 
anomalous dimensions of the operators, $\mathcal{O}_{1}-\mathcal{O}_{5}$. The anomalous dimension of $\mathcal{O}_{1}$ is well-known [99] and is $\mu d C_{1} / d \mu=\alpha_{s} C_{1} / \pi$. The other anomalous dimensions may be found in Ref. [98] and have been independently verified by the author (also see Ref. [98] for a more general analysis of QCD corrections to the SUSY contributions to $K-\bar{K}$ mixing). These authors, however, choose to RG scale to $\mu_{\text {had }}$, defined by $\alpha_{s}\left(\mu_{\text {had }}\right)=1$. The validity of the perturbation expansion is questionable at this scale; here instead the RG scaling is stopped at $900 \mathrm{MeV}$, where $\alpha_{s}(900 \mathrm{MeV}) \sim .4$. The result is

$$
\begin{aligned}
& C_{1}\left(\mu_{\text {had }}\right)=\kappa_{1} C_{1}\left(M_{S}\right) \\
& \tilde{C}_{1}\left(\mu_{\text {had }}\right)=\kappa_{1} \tilde{C}_{1}\left(M_{S}\right) \\
& C_{4}\left(\mu_{\text {had }}\right)=\kappa_{4} C_{4}\left(M_{S}\right)+\frac{1}{3}\left(\kappa_{4}-\kappa_{5}\right) C_{5}\left(M_{S}\right) \\
& C_{5}\left(\mu_{\text {had }}\right)=\kappa_{5} C_{5}\left(M_{S}\right)
\end{aligned}
$$

where

$$
\begin{aligned}
\kappa_{1}= & \left(\frac{\alpha_{s}\left(m_{c}\right)}{\alpha_{s}(900 \mathrm{MeV})}\right)^{6 / 27}\left(\frac{\alpha_{s}\left(m_{b}\right)}{\alpha_{s}\left(m_{c}\right)}\right)^{6 / 25}\left(\frac{\alpha_{s}\left(m_{t}\right)}{\alpha_{s}\left(m_{b}\right)}\right)^{6 / 23} \\
& \times\left(\frac{\alpha_{s}\left(\mu_{G}\right)}{\alpha_{s}\left(m_{t}\right)}\right)^{6 / 21}\left(\frac{\alpha_{s}\left(M_{S}\right)}{\alpha_{s}\left(\mu_{G}\right)}\right)^{6 /\left(9+\left(n_{5}+3 n_{10}\right) / 2\right)} \\
\kappa_{4}= & \kappa_{1}^{-4} \\
\kappa_{5}= & \kappa_{1}^{1 / 2}
\end{aligned}
$$

The effective Lagrangian at the hadronic scale is then

$$
\mathcal{L}_{\text {eff }}=\frac{\alpha_{s}^{2}\left(M_{S}\right)}{216 M_{S}^{2}}\left(-22\left(\delta_{L L}^{d}\right)^{2} \kappa_{1} \mathcal{O}_{1}-22\left(\delta_{R R}^{d}\right)^{2} \kappa_{1} \tilde{\mathcal{O}}_{1}+\delta_{L L}^{d} \delta_{R R}^{d}\left(\frac{8}{3}\left(4 \kappa_{4}+5 \kappa_{5}\right) \mathcal{O}_{4}-40 \kappa_{5} \mathcal{O}_{5}\right)+\text { h.c. }\right) .
$$


The SUSY contribution to the $K-\bar{K}$ mass difference is

$$
\left(\Delta m_{K}\right)_{S U S Y}=2 \operatorname{Re}<K\left|\mathcal{L}_{e f f}\right| \bar{K}>
$$

The relevant matrix elements (with bag factors set to 1) are

$$
\begin{aligned}
& \left\langle K\left|\mathcal{O}_{1}\right| \bar{K}>=\frac{1}{3} m_{K} f_{K}^{2}\right. \\
& <K\left|\mathcal{O}_{4}\right| \bar{K}>=\left(\frac{1}{24}+\frac{1}{4}\left(\frac{m_{K}}{m_{s}+m_{d}}\right)^{2}\right) m_{K} f_{K}^{2} \\
& <K\left|\mathcal{O}_{5}\right| \bar{K}>=\left(\frac{1}{8}+\frac{1}{12}\left(\frac{m_{K}}{m_{s}+m_{d}}\right)^{2}\right) m_{K} f_{K}^{2}
\end{aligned}
$$

in the vacuum insertion approximation. The values [36] $m_{K}=497 \mathrm{MeV}, f_{K}=160$ $\mathrm{MeV}, m_{s}=150 \mathrm{MeV},\left(\Delta m_{K}\right)_{\exp }=3.5 \times 10^{-12} \mathrm{MeV}$, and $\alpha_{s}\left(M_{Z}\right)=0.118$ are used. This gives $\alpha_{s}\left(m_{b}\right)=0.21, \alpha_{s}\left(m_{c}\right)=0.29$ and $\alpha_{s}(900 \mathrm{MeV})=0.38$ using the one-loop RG evolution. Once values for $\left(n_{5}, n_{10}, \delta_{L L}^{d}, \delta_{R R}^{d}\right)$ are specified, a minimum value for $M_{S}$ is obtained by requiring that $\left(\Delta m_{K}\right)_{S U S Y}=\left(\Delta m_{K}\right)_{\text {exp }}$. In the case that both $\delta_{R R} \neq 0$ and $\delta_{L L} \neq 0$, both the left-handed and right-handed squarks are assumed to be heavy, so that $\left(n_{5}, n_{10}\right)=(2,2)$. In this case only the dominant contribution to $\Delta m_{K}$, which is $\sim \delta_{L L}^{d} \delta_{R R}^{d}$, is required to equal the measured value of $\Delta m_{K}$. If $\delta_{R R} \neq 0$ and $\delta_{L L}=0$, only the right-handed squarks are assumed to be heavy, and thus $\left(n_{5}, n_{10}\right)=(2,0)$. Similarly, if $\delta_{L L} \neq 0$ and $\delta_{R R}=0$ then $\left(n_{5}, n_{10}\right)=(0,2)$. Limits are given in Tables 6.1 and 6.2 for some choices of these parameters. These results agree with Ref. [98] for the same choice of input parameters. For comparison, the limits gotten by neglecting the QCD corrections are also presented in Tables 6.1 and 6.2. Here $\delta_{L L}^{d}\left(\delta_{R R}^{d}\right)=(i) 1,(i i)$ 0.22 , (iii) 0.1 , and (iv) 0.04 are considered. These correspond to: (i) no mixing 


\begin{tabular}{||l|l|l||}
\hline$\sqrt{\operatorname{Re}\left(\delta_{L L}^{d} \delta_{R R}^{d}\right)}$ & $\left(n_{5}, n_{10}\right)=(2,2)$ & $\left(n_{5}, n_{10}\right)=(2,2)$ \\
\hline & QCD incl. & no QCD \\
\hline 1 & $182 \mathrm{TeV}$ & $66 \mathrm{TeV}$ \\
\hline 0.22 & $40 \mathrm{TeV}$ & $15 \mathrm{TeV}$ \\
\hline 0.1 & $18 \mathrm{TeV}$ & $7.3 \mathrm{TeV}$ \\
\hline 0.04 & $7.3 \mathrm{TeV}$ & $3.1 \mathrm{TeV}$ \\
\hline
\end{tabular}

Table 6.1: Minimum values for heavy scalar masses $M_{S}$ obtained from the measured value of $\Delta m_{K}$ assuming $M_{3}^{2} / M_{S}^{2} \ll 1$. The limits labeled 'QCD incl.' include QCD corrections as discussed in the text. Those labeled as 'no QCD' do not.

and no degeneracy; (ii) Cabibbo-like mixing; (iii) Cabibbo-like mixing and $\sim 0.5$ degeneracy; and (iv) Cabibbo-like mixing and Cabibbo-like degeneracy. Only cases $(i),(i i)$ and (iii) are expected to be relevant if the supersymmetric flavor problem is resolved by decoupling the first two generation scalars. Note that for $\left(n_{5}, n_{10}\right)=(2,0)$, Table 6.2 implies that $M_{S}$ must be larger than $\sim 30 \mathrm{TeV}$ if it is assumed there is no small mixing or degeneracy $\left(\delta_{R R}^{d}=1\right)$ between the first two generation scalars.

The limits obtained from the measured rate of $C P$ violation are now briefly discussed. Recall that the $C P$ violating parameter $\epsilon$ is approximately

$$
|\epsilon| \sim \frac{|\operatorname{Im}<K| \mathcal{L}_{e f f}|\bar{K}>|}{\sqrt{2} \Delta m_{K}}
$$

and its measured value is $|\epsilon| \sim\left|\eta_{00}\right|=2.3 \times 10^{-3}$ [36]. In this case, the small value 


\begin{tabular}{||l|l|l||}
\hline $\operatorname{Re}\left(\delta_{R R}^{d}\right)\left(\delta_{L L}^{d}=0\right)$ & $\left(n_{5}, n_{10}\right)=(2,0)$ & $\left(n_{5}, n_{10}\right)=(2,0)$ \\
\hline & QCD incl. & no QCD \\
\hline 1 & $30 \mathrm{TeV}$ & $38 \mathrm{TeV}$ \\
\hline 0.22 & $7.2 \mathrm{TeV}$ & $8.9 \mathrm{TeV}$ \\
\hline 0.1 & $3.4 \mathrm{TeV}$ & $4.1 \mathrm{TeV}$ \\
\hline 0.04 & $1.4 \mathrm{TeV}$ & $1.7 \mathrm{TeV}$ \\
\hline
\end{tabular}

Table 6.2: Minimum values for heavy scalar masses $M_{S}$ obtained from the measured value of $\Delta m_{K}$ assuming $M_{3}^{2} / M_{S}^{2} \ll 1$. The limits labeled as 'QCD incl.' include QCD corrections as discussed in the text. Those labeled as 'no QCD' do not. The limits for $\left(n_{5}, n_{10}\right)=(0,2)$ obtained by $\delta_{L L}^{d} \leftrightarrow \delta_{R R}^{d}$ are similar and not shown. 
of $\epsilon$ implies either that the phases appearing in the soft scalar mass matrix are extremely tiny, or that the masses of the heavy scalars are larger than the limits given in Tables 6.1 and 6.2. In the case where the phases are $O(1)$, $\operatorname{Im}<K\left|\mathcal{L}_{\text {eff }}\right| \bar{K}>\sim \operatorname{Re}<K\left|\mathcal{L}_{\text {eff }}\right| \bar{K}>$ and thus the stronger constraint on $M_{S}$ is obtained from $\epsilon$ and not $\Delta m_{K}$, for the same choice of input parameters. In particular, the constraint from $C P$ violation increases the minimum allowed value of $M_{S}$ by a factor of $1 / \sqrt{2 \sqrt{2} \epsilon} \sim 12.5$. This significantly increases the minimum value of the initial light scalar masses that is allowed by the positivity requirement.

\subsubsection{RGE analysis}

The values of the soft masses at the weak scale are determined by the RG evolution. In the $\overline{D R}^{\prime}$ scheme $[101,102,103]$, the RG equations for the light scalar masses are, including the gaugino, $A$-term and $\lambda_{t}$ contributions at the one-loop level and the heavy scalar contribution at the two-loop level [104],

$$
\begin{aligned}
\frac{d}{d t} m_{i}^{2}(t=\ln \mu)= & -\frac{2}{\pi} \sum_{A} \alpha_{A}(t) C_{A}^{i} M_{A}^{2}(t)+\frac{4}{16 \pi^{2}} \sum_{A} C_{A}^{i} \alpha_{A}^{2}(t)\left(n_{5} m_{5}^{2}+3 n_{10} m_{10}^{2}\right) \\
& +\frac{8}{16 \pi^{2}} \frac{3}{5} Y_{i} \alpha_{1}(t)\left(\frac{4}{3} \alpha_{3}(t)-\frac{3}{4} \alpha_{2}(t)-\frac{1}{12} \alpha_{1}(t)\right)\left(n_{5} m_{5}^{2}-n_{10} m_{10}^{2}\right) \\
& +\frac{\eta_{i} \lambda_{t}^{2}(t)}{8 \pi^{2}}\left(m_{H_{u}}^{2}(t)+m_{\tilde{u}_{3}^{c}}^{2}(t)+m_{\tilde{Q}_{3}}^{2}(t)+A(t)^{2}\right) \\
& +\frac{6}{5} \frac{1}{4 \pi} Y_{i} \alpha_{1}(t) \operatorname{Tr} Y m^{2}(t)
\end{aligned}
$$

with $\eta=(3,2,1)$ for $\tilde{f}_{i}=H_{u}, \tilde{t}^{c}, \tilde{t}$, respectively, and zero otherwise. For simplicity it is assumed that $M_{A, 0} / \alpha_{A, 0}$ are all equal at $M_{S U S Y}$. The initial value of the gluino mass, $M_{3,0}$, is then chosen to be the independent parameter. To avoid a large 
Fayet-Illiopoulus $D$-term at the one-loop level, the heavy scalars are assumed to form complete $S U(5)$ representations[96, 92]. $S U(5)$ normalization for the $U(1)$ coupling constant is used and $Q=T_{3}+Y$. Finally, $C_{A}^{i}$ is the quadratic Casimir for the gauge group $G_{A}$ that is $4 / 3$ and $3 / 4$ for the fundamental representations of $S U(3)$ and $S U(2)$, and $3 / 5 Y_{i}^{2}$ for the $U(1)$ group. The cases $\left(n_{5}, n_{10}\right)=(\mathrm{I})(2,2)$, (II) $(2,0)$, (III) $(0,2)$ are considered. The results for the case $(3,0)$ is obtained, to a good approximation, from Case (II) by a simple scaling, and it is not discussed any further.

Inspection of Eqn.(6.12) reveals that in RG scaling from a high scale to a smaller scale the two-loop gauge contribution to the soft masses is negative, and that of the gauginos is positive. The presence of the large $\lambda_{t}$ Yukawa coupling in the RGE drives the value of the stop soft mass squared even more negative. This effect increases the bound on the initial value for the stop soft masses and is included in this analysis. In this analysis the top quark mass in $\overline{M S}$ scheme is fixed at $167 \mathrm{GeV}$.

In the MSSM there is an extra parameter, $\tan \beta$, which is the ratio of the vacuum expectations values of the Higgs fields that couple to the up-type and down-type quarks respectively. Electroweak symmetry breaking then determines the top quark mass to be $m_{t}=\lambda_{t} / \sqrt{2} v \sin \beta$ with $v \sim 247 \mathrm{GeV}$. In this analysis the regime of small to moderate $\tan \beta$ is considered, so that all Yukawa couplings other than $\lambda_{t}$ are neglected in the RG evolution. In this approximation the numerical results for $\tilde{f}_{i} \neq \tilde{t}$ or $\tilde{t}^{c}$ are independent of $\tan \beta$. In the numerical analysis of 
sections 6.3 and $6.4 \tan \beta=2.2$ is considered. In section $6.5 \tan \beta=10$ is also considered.

In the case of low-energy supersymmetry breaking, the scale $M_{S U S Y}$ is not much larger than the mass scale of the heavy scalars. Then the logarithm $\sim \ln \left(M_{S U S Y} / M_{S}\right)$ that appears in the solution to the previous $R G$ equations is only $O(1)$. In this case the finite parts of the two-loop diagrams may not be negligible and should be included in the analysis. These finite parts are used to estimate the size of the two-loop heavy scalar contribution in an actual model.

The full two-loop expression for the soft scalar mass at a renormalisation scale $\mu_{R}$ is $m_{\text {full }}^{2}\left(\mu_{R}\right)=m_{\overline{D R}^{\prime}}^{2}\left(\mu_{R}\right)+m_{\text {finite }}^{2}\left(\mu_{R}\right)$, where $m_{\overline{D R}^{\prime}}^{2}\left(\mu_{R}\right)$ is the solution to the RG equation in $\overline{D R}^{\prime}$ scheme, and $m_{\text {finite }}^{2}\left(\mu_{R}\right)$ is the finite part of the one-loop and two-loop diagrams, also computed in $\overrightarrow{D R}^{\prime}$ scheme. The finite parts of the two-loop diagrams that contain internal heavy scalars are computed in Appendix $\mathrm{B}$ and the details are given therein. The answer for these two-loop finite parts is (assuming all heavy scalars are degenerate with common mass $M_{S}^{2}$ )

$$
\begin{aligned}
m_{i, f i n i t e}^{2}\left(\mu_{R}\right)= & -\frac{1}{8}\left(\ln (4 \pi)-\gamma+\frac{\pi^{2}}{3}-2-\ln \left(\frac{M_{S}^{2}}{\mu_{R}^{2}}\right)\right) \\
& \times \sum_{A}\left(\frac{\alpha_{A}\left(\mu_{R}\right)}{\pi}\right)^{2}\left(n_{5}+3 n_{10}\right) C_{A}^{i} M_{S}^{2} \\
& -\frac{3}{5} \frac{1}{16 \pi^{2}} \alpha_{1}\left(\mu_{R}\right)\left(n_{5}-n_{10}\right) Y_{i}\left(6-\frac{2}{3} \pi^{2}+2(\ln (4 \pi)-\gamma)-4 \ln \left(\frac{M_{S}^{2}}{\mu_{R}^{2}}\right)\right) \\
& \times\left(\frac{4}{3} \alpha_{3}\left(\mu_{R}\right)-\frac{3}{4} \alpha_{2}\left(\mu_{R}\right)-\frac{1}{12} \alpha_{1}\left(\mu_{R}\right)\right) M_{S}^{2}
\end{aligned}
$$

where the gaugino and fermion masses are neglected. Since the $\overline{D R}^{\prime}$ scheme is used to compute the finite parts of the soft scalar masses, the limits obtained on the initial masses are only valid, strictly speaking, in this scheme. This is 
especially relevant for the case of low scale SUSY breaking. So while these finite parts should be viewed as semi-quantitative, they should suffice for a discussion of the fine tuning that results from the limit on the bare stop mass. For the case of high scale SUSY breaking, the RG logarithm is large and so the finite parts are not that important.

The numerical analysis for either low-energy or high-energy supersymmetry breaking is described as follows.

The RG equations are evolved from the scale $M_{S U S Y}$ to the scale at which the heavy scalars are decoupled. This scale is denoted by $\mu_{S}$ and should be $O\left(M_{S}\right)$. The RG scaling of the heavy scalars is neglected. At this scale the finite parts of the two-loop diagrams are added to $m_{\tilde{f}_{i}}^{2}\left(\mu_{S}\right)$. Note that since the two-loop information included in the RG analysis is the leading $O\left(M_{S}^{2}\right)$ effect, it is sufficient to only use tree-level matching at the scale $\mu_{S}$. Since the heavy scalars are not included in the effective theory below $M_{S}$ and do not contribute to the gauge coupling beta functions, the numerical results contain an implicit dependence on the number of heavy scalars. This results in a smaller value for $\alpha_{3}\left(\mu_{S}\right)$ compared to its value if insiead all the scalars have $\mathrm{a} \sim 1 \mathrm{TeV}$ mass. This tends to weaken the constraint, and so it is included in our analysis ${ }^{3}$. The soft masses are then evolved using the one-loop RGE to the mass scale at which the gluinos are decoupled. This scale is fixed to be $\mu_{G}=1 \mathrm{TeV}$.

\footnotetext{
${ }^{3}$ This is the origin of a small numerical discrepancy of $\sim 10 \%$ between these results and the analysis of [97] in the approximation $\lambda_{t}=0$.
} 
A constraint on the initial value of the soft masses is obtained by requiring that at the weak scale the physical scalar masses are positive. The experimental limit is $\sim 70 \mathrm{GeV}$ for charged or colored scalars[100]. The physical mass of a scalar is equal to the sum of the soft scalar mass, the electro-weak $D$-term, the supersymmetric contribution, and some finite one-loop and two-loop contributions. As mentioned in the previous paragraph, in the effective theory below $M_{S}$ the finite two-loop part from the heavy scalars is included in value of the soft scalar mass of the light sparticles at the boundary, defined at $\mu_{R}=\mu_{S} \sim M_{S}$. The finite oneloop contributions are proportional to the gaugino and other light scalar masses, and are smaller than the corresponding logarithm that is summed in $\tilde{m}_{i}^{2}\left(\mu_{R}\right)$. So these finite one-loop parts are neglected. Further, the electroweak $D$-terms are less than $70 \mathrm{GeV}$. For the scalars other than the stops, the supersymmetric contribution is negligible. In what follows then, $\tilde{m}_{i}^{2}\left(\mu_{G}\right)>0$ is required for scalars other than the stops. The discussion with the stops is complicated by both the large supersymmetric contribution, $m_{t}^{2}$, to the physical mass and by the $L-R$ mixing between the gauge eigenstates. This mixing results in a state with mass squared less than $\min \left(m_{\tilde{t}}^{2}+m_{t}^{2}, m_{\tilde{t}^{c}}^{2}+m_{t}^{2}\right)$, so it is a conservative assumption to require that for both gauge eigenstates the value of $m_{\tilde{t}_{i}}^{2}+m_{t}^{2}$ is larger than the experimental limit. This implies that $m_{\tilde{t}_{i}}^{2} \geq(70 \mathrm{GeV})^{2}-(175 \mathrm{GeV})^{2}=-(160$ $\mathrm{GeV})^{2}$. Instead, in what follows $m_{\tilde{t}_{i}}^{2} \geq 0$ is required. This results in an error that is $(160 \mathrm{GeV})^{2} / 2 m_{\tilde{t}_{i, 0}} \approx 26 \mathrm{GeV}$ if the constraint obtained by neglecting $m_{t}$ is $\sim 1$ $\mathrm{TeV}$. For the parameter range of interest it will be shown that the limit on the 
initial squark masses is $\sim 1 \mathrm{TeV}$, so this approximation is consistent.

Then the above two analyses are combined as follows. The $\Delta m_{K}$ constraints of section 6.2.1 determine a minimum value for $M_{S}$ once some theoretical preference for the $\delta$ 's is given. Either a natural value for the $\delta$ 's is predicted by some model, or the $\delta$ 's are arbitrary and chosen solely by naturalness considerations. Namely, in the latter case the fine tuning to suppress $\Delta m_{K}$ is roughly $2 / \delta$. Further, a model may also predict the ratio $M_{3} / M_{S}$. Otherwise, Eqns.(6.1) and (6.2) may be used as a rough guide to determine an upper value for $M_{3}$, based upon naturalness considerations of the $Z$ mass. Without such a limitation, the positivity requirements are completely irrelevant if the bare gluino mass is suffuciently large; but then the $Z$ mass is fine tuned. Using these values of $M_{3}$ and $M_{S}$, the RGE analysis gives a minimum value for the initial stop masses which is consistent with $\Delta m_{K}$ and positivity of the soft masses. This translates into some fine tuning of the $Z$ mass, which is then roughly quantified by Eqns.(6.1) and (6.2).

Finally, this analysis may also be extended to include models that contain a Fayet-Illiopoulos hypercharge $D$-term, $\zeta_{D}$, at the tree-level. The effect of the $D$-term is to shift the soft scalar masses, $m_{i, 0}^{2} \rightarrow \tilde{m}_{i, 0}^{2}=m_{i, 0}^{2}+Y_{i} \zeta_{D}$. In this case, the positivity analysis applies to $\tilde{m}_{i, 0}^{2}$, rathe than $m_{i, 0}^{2}$.

\subsection{Low Energy Supersymmetry Breaking}

This section investigates the positivity requirement within a framework that satisifes both of the following: (i) supersymmetry breaking is communicated to 
the visible sector at low energies; and (ii) multi-TeV scale soft masses, $M_{S}$, are generated for some of the first two generation scalars. This differs from the usual low-energy supersymmetry breaking scenario in that here $M_{S}^{2} \gg m_{\tilde{t}_{i}, 0}^{2}$ is assumed. In the absence of a specific model, however, it is difficult to obtain from the positivity criterion robust constraints on the scalar spectra for the following reasons. At the scale $M_{S U S Y}$ it is expected that, in addition to the heavy scalars of the MSSM, there are particles that may have SM quantum numbers and supersymmetry breaking mass parameters. All these extra states contribute to the soft scalar masses of the light particles. The sign of this contribution depends on, among other things, whether the soft mass squared for these additional particles is positive or negative - clearly very model-dependent. The total two-loop contribution to the light scalar masses is thus a sum of a model-dependent part and a model independent part. By considering only the model-independent contribution only isolated one particular contribution to the total value of the soft scalar masses near the supersymmetry breaking scale has been isolated. However, these results are used to estimate the typical size of the finite parts in an actual model. That is, if in an actual model the sign of the finite parts is negative and its size is of the same magnitude as in Eqn.(6.13), the constraint in that model is identical to the constraint obtained here. The constraint for other values for the finite parts is then obtained by a simple rescaling.

Before discussing the numerical results, the size of the finite contributions are estimated in order to illustrate the problem. Substituting $M_{S} \sim 25 \mathrm{TeV}, \alpha_{3}(25$ 
$\mathrm{TeV}) \sim 0.07$ and $\alpha_{1}(25 \mathrm{TeV}) \sim 0.018$ into Eqn. $(6.13)$ gives

$$
\delta m_{\tilde{q}}^{2} \approx-(410 \mathrm{GeV})^{2}\left(n_{5}+3 n_{10}\right)\left(\frac{M_{S}}{25 \mathrm{TeV}}\right)^{2}
$$

for squarks, and

$$
\delta m_{\tilde{e}^{c}}^{2} \approx-\left(\left(n_{5}+3 n_{10}\right)(70 \mathrm{GeV})^{2}+\left(n_{5}-n_{10}\right)(100 \mathrm{GeV})^{2}\right)\left(\frac{M_{S}}{25 \mathrm{TeV}}\right)^{2}
$$

for the right-handed selectron. The negative contribution is large if $M_{S} \sim 25 \mathrm{TeV}$. For example, if $n_{5}=n_{10}=2$ then $\delta m_{\tilde{e}^{c}}^{2} \approx-(200 \mathrm{GeV})^{2}$ and $\delta m_{\tilde{q}}^{2} \approx-(1.2 \mathrm{TeV})^{2}$. If $n_{5}=2, n_{10}=0$, then $\delta m_{\tilde{e}^{c}}^{2} \approx-(170 \mathrm{GeV})^{2}$ and $\delta m_{\tilde{q}}^{2} \approx-(580 \mathrm{GeV})^{2}$.

In this low-energy supersymmetry breaking scenario, it is expected that $M_{S U S Y} \sim M_{S}$. In the numerical analysis $M_{S U S Y}=\mu_{S}$ is assumed since the actual messenger scale is not known. The scale $\mu_{S}$ is chosen to be $50 \mathrm{TeV}$. At the scale $\mu_{S}=50 \mathrm{TeV}$ the $\mu_{S}$-independent parts of Eqn. (6.13) are added to the initial value of the soft scalar masses. The soft masses are then evolved using the RG equations (not including the two-loop contribution) to the scale $\mu_{G}=1 \mathrm{TeV}$.

First, the constraints the positivity requirement imply for $\tilde{f}_{i} \neq \tilde{t}_{L}$ or $\tilde{t}_{R}$ are discussed. In this case $m_{\tilde{f}_{i}}^{2}$ is renormalized by $M_{3,0}^{2}, M_{S}^{2}, m_{\tilde{f}_{i}, 0}^{2}$ and the initial value of $\operatorname{Tr} Y m^{2} \equiv D_{Y, 0}$. A numerical computation gives

$$
\begin{aligned}
m_{\tilde{f}_{i}}^{2}\left(\mu_{G}\right)= & m_{\tilde{f}_{i}, 0}^{2}+\left(0.243 C_{3}^{i}+0.0168 C_{2}^{i}+0.00156 Y_{i}^{2}\right) M_{3,0}^{2}+c_{D} \times 10^{-3} Y_{i} D_{Y, 0} \\
& -\left(0.468 C_{3}^{i}+0.095 C_{2}^{i}+0.0173 Y_{i}^{2}\right) \frac{1}{2}\left(n_{5}+3 n_{10}\right) \times 10^{-3} M_{S}^{2} \\
& -\left(n_{5}-n_{10}\right)\left(\left(-0.00058+0.0016\left(n_{5}+3 n_{10}\right)\right) M_{S}^{2}-0.925 M_{3,0}^{2}\right) Y_{i} \times 10^{-3} \\
& -0.0174\left(n_{5}-n_{10}\right) Y_{i} \times 10^{-3} M_{S}^{2}
\end{aligned}
$$


where the strongest dependence on $\left(n_{5}, n_{10}\right)$ has been isolated. The coefficient appearing in front of $D_{Y, 0}$ is $c_{D}=-6$. The numerical coefficients in Eqn.(6.16) also depend on $\left(n_{5}, n_{10}\right)$ and the numbers presented in Eqn.(6.16) are for $\left(n_{5}, n_{10}\right)=$ $(2,0)$. This sensitivity is, however, only a few percent between the four cases under consideration here ${ }^{4}$. Requiring positivity of the soft scalar masses directly constrains $m_{\tilde{f}_{i}, 0}^{2} / M_{S}^{2}$ and $M_{3,0}^{2} / M_{S}^{2}$.

The value of $D_{Y, 0}$ depends on the spectrum at the supersymmetry breaking scale, and is therefore model-dependent. To obtain model-independent constraints from the positivity requirement, the combination $\tilde{m}_{\tilde{f}_{i}, 0}^{2} \equiv m_{\tilde{f}_{i}, 0}^{2}+c_{D} \times$ $10^{-3} \times Y_{i} D_{Y, 0}$ is constrained. Only this combination appears in the weak-scale value for the scalar mass of $\tilde{f}_{i}$. The numerical effect is small, since with $D_{Y, 0} \sim O\left(m_{\tilde{f}_{i}, 0}^{2}\right)$, the coefficient of $m_{\tilde{f}_{i}, 0}^{2}$ is shifted from 1 to $\sim(1-6) \times 10^{-3} Y_{i}$.

The positivity requirement $\tilde{m}_{\tilde{f}_{i}}^{2}$ for $\tilde{f}_{i} \neq \tilde{t}$ or $\tilde{t}^{c}$ is given in Figure 6.1 for different values of $n_{5}$ and $n_{10}$. That is, in figure 6.1 the minimum value of $\tilde{m}_{\tilde{f}_{i}, 0} / M_{S}$ required to keep the soft masses positive at the scale $\mu_{G}$ is plotted versus $M_{3,0} / M_{S}$. From these Figures it is seen that the positivity criterion is weakest for $n_{5}=2$ and $n_{10}=0$. This is expected since in this case the heavy particle content is the smallest. Note that even in this "most minimal" scenario the negative contribution to the masses are rather large. In particular, Figure 6.1 implies that for $\left(n_{5}=2, n_{10}=0\right)$ and $M_{S} \sim 25 \mathrm{TeV}, \delta m_{\tilde{e}^{c}}^{2} \approx-(190 \mathrm{GeV})^{2}$ for $M_{3,0}$ as large as $1 \mathrm{TeV}$. In this case it is the two-loop contribution from the hypercharge $D$-term that is responsible for

\footnotetext{
${ }^{4}$ This dependence is included in Figure 6.1.
} 
the large negative mass squared. In the case $\left(n_{5}, n_{10}\right)=(2,2)$, Figure 6.1 implies that for $M_{S} \sim 25 \mathrm{TeV}, \delta m_{\tilde{e}^{c}}^{2} \approx-(210 \mathrm{GeV})^{2}$ and $\delta m_{\tilde{b}^{c}}^{2} \approx-(1.1 \mathrm{TeV})^{2}$ for $M_{3,0}$ as large as $1 \mathrm{TeV}$.

The positivity requirement for the stops is obtained next. In this case it is not possible to directly constrain the boundary values of the stops for the following simple reason. There are only two positivity constraints, whereas the values of $m_{\tilde{t}}^{2}\left(\mu_{G}\right)$ and $m_{\tilde{t}^{c}}^{2}\left(\mu_{G}\right)$ are functions of the three soft scalar masses $m_{\tilde{t}, 0}^{2}, m_{\tilde{t}^{c}, 0}^{2}$ and $m_{H_{u}, 0^{0}}^{2}$. To obtain a limit some theoretical assumptions must be made to relate the three initial soft scalar masses.

The numerical solutions to the RG equations for $\tan \beta=2.2$ and $\left(n_{5}, n_{10}\right)=$ $(2,0)$ are

$$
\begin{aligned}
m_{\tilde{t}}^{2}\left(\mu_{G}\right)= & -0.0303 A_{t}^{2}+0.00997 A_{t} M_{3,0}+0.322 M_{3,0}^{2}+c_{D} \times \frac{1}{6} \times 10^{-3} D_{Y, 0} \\
& -0.0399\left(m_{H_{u}, 0}^{2}+m_{\tilde{t}^{c}, 0}^{2}\right)+0.960 m_{\tilde{t}, 0}^{2}-0.000645 c_{L} M_{S}^{2} \\
m_{\tilde{t}^{c}}^{2}\left(\mu_{G}\right)= & -0.0606 A_{t}^{2}+0.0199 A_{t} M_{3,0}+0.296 M_{3,0}^{2}+c_{D} \times \frac{-2}{3} \times 10^{-3} D_{Y, 0} \\
& +0.920 m_{\tilde{t}^{c}, 0}^{2}-0.0797\left(m_{H_{u}, 0}^{2}+m_{\tilde{t}, 0}^{2}\right)-0.000492 c_{R} M_{S}^{2} \\
m_{\tilde{H}_{u}}^{2}\left(\mu_{G}\right)= & -0.0909 A_{t}^{2}+0.0299 A_{t} M_{3,0}-0.0289 M_{3,0}^{2}+c_{D} \times \frac{1}{2} \times 10^{-3} D_{Y, 0} \\
& +0.880 m_{H_{u}, 0}^{2}-0.119\left(m_{\tilde{t}, 0}^{2}+m_{\tilde{t}^{c}, 0}^{2}\right)+0.0000719 c_{H} M_{S}^{2} .
\end{aligned}
$$

The numerical coefficients other than that of $M_{S}$ do not vary more than a few percent between the different values for $\left(n_{5}, n_{10}\right)$, and thus this dependence is not shown. The values of the $M_{S}$ coefficient are $\left(c_{L}, c_{R}, c_{H}\right)=(1,1,1)$, $(3.62,3.84,4.59),(2.78,3.04,3.92)$, for $\left(n_{5}, n_{10}\right)=(2,0),(2,2)$ and $(0,2)$, respec- 

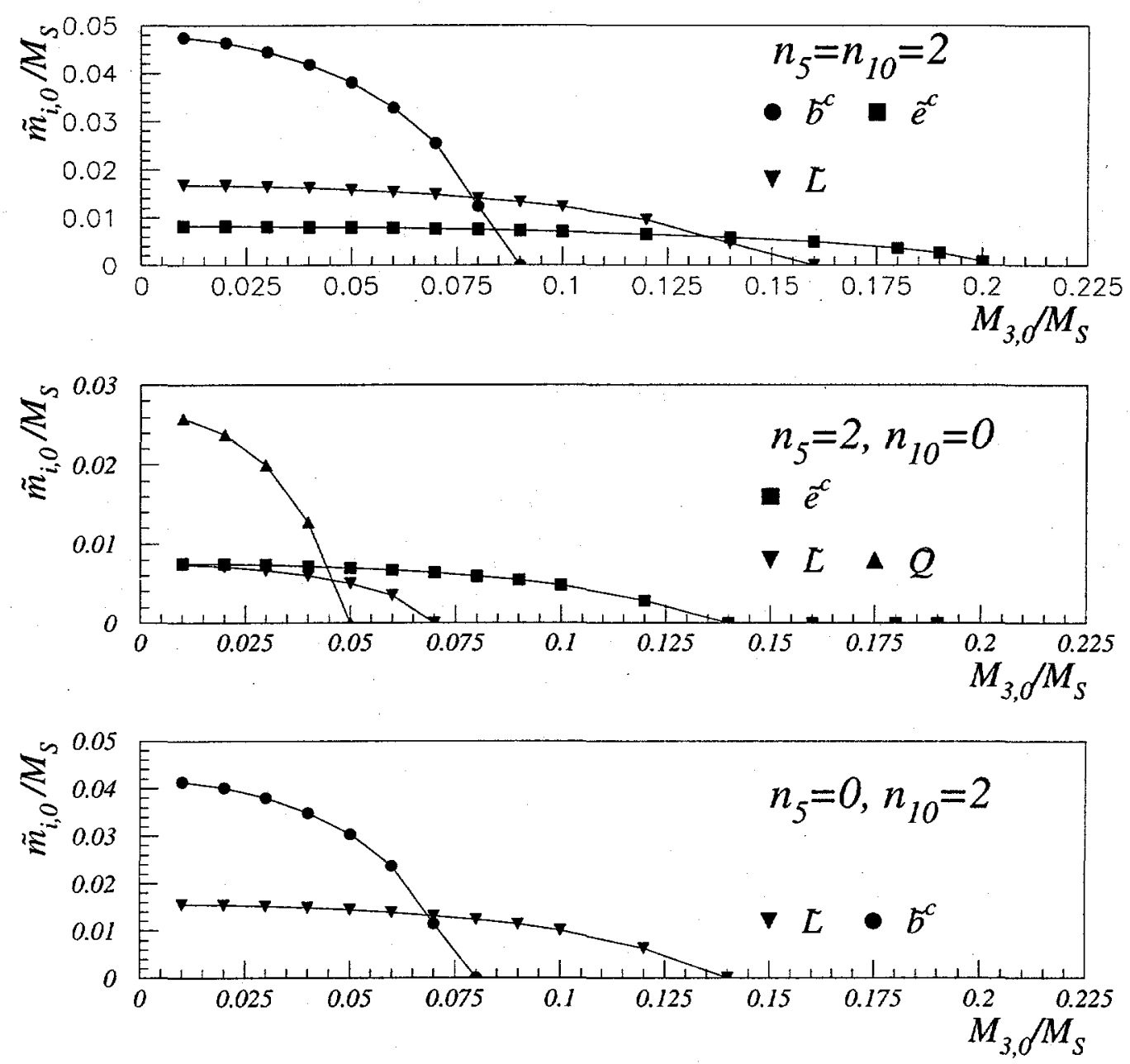

Figure 6.1: Limits for $m_{\tilde{f}_{i}, 0} / M_{S}$ from the requirement that the masses squared are positive at the weak scale, for low-energy supersymmetry breaking. The regions below the curves are excluded. For the case $(2,0)$, the limits for the other squarks are very similar to that for $\tilde{Q}$ and are therefore not shown. 
tively. Also, $c_{D}=-6$. Inspecting Eqns.(6.1) and (6.2) implies that to keep $m_{Z}^{2}$ fine tuned at less than $1 \%(\Delta \leq 100)$ in each of the bare parameters, the following must be satisfied : $\mu \lesssim 460 \mathrm{GeV} ; M_{3,0} \lesssim 2.3 \mathrm{TeV} ; m_{\tilde{t}, 0} \leqslant 1.7 \mathrm{TeV} ; m_{5} \lesssim 80 \mathrm{TeV}$ and $m_{10} \lesssim 50 \mathrm{TeV}$ for $\left(n_{5}, n_{10}\right)=(2,2)$. Finally, for other values of these parameters the fine tuning increases as $\Delta=100 \times \tilde{m}^{2} / \tilde{m}_{0}^{2}$, where $\tilde{m}_{0}$ is the value of $\tilde{m}$ that gives $\Delta=100$.

It is possible to show, using the fact that $Y_{H_{u}}+Y_{Q}+Y_{u^{c}}=0$, that the solutions in Eqn.(6.17) are unchanged if $m_{i, 0}^{2}$ is replaced with $\tilde{m}_{i, 0}^{2}=m_{i, 0}^{2}+c_{D} \times 10^{-3} Y_{i} D_{Y, 0}$ and set $D_{Y, 0}=0$. In what follows then, the posivitity analysis is used to constrain $\tilde{m}_{i, 0}^{2}$ for the stops. The cifference between $\tilde{m}_{i, 0}^{2}$ and $m_{i, 0}^{2}$ is small, though, owing to the small coefficient appearing in front of $D_{Y, 0}$. In the remainder of this section the tilde on $\tilde{m}_{i, 0}^{2}$ will be removed to simplify the notation.

To constrain the initial values of the stop masses only gauge-mediated supersymmetry breaking mass relations are considered. An inspection of Eqn.(6.17) reveals that to naturally break electroweak symmetry a small hierarchy $m_{\bar{t}_{i}, 0}^{2}>m_{H_{u}, 0}^{2}$ is required. This is naturally provided by gauge-mediated boundary conditions ${ }^{5}$. The relations between the soft scalar masses when supersymmetry breaking is communicated to the visible sector by gauge messengers are [11]

$$
m_{i, 0}^{2}=\frac{3}{4} \sum_{A} C_{A}^{i} \frac{\alpha_{A}^{2}\left(M_{S U S Y}\right)}{\alpha_{3}^{2}\left(M_{S U S Y}\right)+\alpha_{1}^{2}\left(M_{S U S Y}\right) / 5} m_{\tilde{t}^{c}, 0}^{2} .
$$

Substituting these relations into Eqn.(6.17) and assuming $A_{t, 0}=0$ determines

\footnotetext{
${ }^{5}$ In fact, low-energy gauge-mediated supersymmetry breaking provides "too much" electroweak symmetry breaking [105].
} 
$m_{\tilde{t}}^{2}\left(\mu_{G}\right)$ and $m_{\tilde{t}^{c}}^{2}\left(\mu_{G}\right)$ as a function of $M_{3,0}, M_{S}^{2}$ and $m_{\tilde{t}^{c}, 0}^{2}$. Figure 6.2 plots the minimum value of $m_{\tilde{t}^{c}, 0} / M_{3,0}$ required to maintain both $m_{\tilde{t}}^{2}\left(\mu_{G}\right) \geq 0$ and $m_{\tilde{t}^{c}}^{2}\left(\mu_{G}\right) \geq 0$.

Another interesting constraint on these class of models is found if it is assumed that the initial masses of all the light fields are related at the supersymmetry breaking scale by some gauge--mediated supersymmetry breaking (GMSB) mass relations, as in Eqn.(6.18). This ensures the degeneracy, as required by the flavor changing constraints, of any light scalars of the first two generations. This is required if, for example, one of $n_{5}$ or $n_{10}$ are zero. Then in the previous limits of $m_{\tilde{f}_{i}, 0}$ for $\tilde{f}_{i} \neq \tilde{t}$ or $\tilde{t}^{c}$, constraints on the initial value of $m_{\tilde{t}^{c}}$ are obtained by relating $m_{\tilde{f}_{i}, 0}$ to $m_{\tilde{t}^{c}, 0}$ using Eqn.(6.18). In this case the slepton masses provide the strongest constraint and they are also shown in Figure 6.2. This result may be understood from the following considerations. The two-loop hypercharge $D$ term contribution to the soft mass is $\sim Y_{i}\left(n_{5}-n_{10}\right) \alpha_{1} \alpha_{3} M_{S}^{2}$ and this has two interesting consequences. The first is that for $n_{5} \neq n_{10}$, the resulting $\delta \tilde{m}^{2}$ is always negative for one of $\tilde{e}^{c}$ or $\tilde{L}$. Thus in this case there is always a constraint on $m_{\hat{t}^{c}}^{2}$ once gauge-mediated boundary conditions are assumed. That this negative contribution is large is seen as follows. The combined tree-level mass and two-loop contribution to the selectron mass is approximately $m_{\tilde{e}^{c}, 0}^{2}-k \alpha_{1} \alpha_{3} M_{S}^{2}$ where $k$ is a numerical factor. Substituting the gauge-mediated relation $m_{\tilde{e}^{c}, 0}^{2} \sim \alpha_{1}^{2} / \alpha_{3}^{2} m_{\tilde{t}^{c}, 0}^{2}$, the combined selectron mass is $\alpha_{1}^{2} / \alpha_{3}^{2}\left(m_{\tilde{t}^{c}, 0}^{2}-k\left(\alpha_{3} / \alpha_{1}\right) \alpha_{3}^{2} M_{S}^{2}\right)$. Since the combined mass of the stop is $\sim m_{\tilde{t}^{c}, 0}^{2}-k^{\prime} \alpha_{3}^{2} M_{S}^{2}$, the limit for $m_{\tilde{t}^{c}, 0}^{2}$ obtained from the positivity requirement for $m_{\tilde{e}^{c}}^{2}$ is comparable or larger than the constraint obtained from 
requiring that $m_{\tilde{t}^{c}}^{2}$ remains positive. For example, with $n_{5}=2, n_{10}=0$ and $M_{S} \sim 25 \mathrm{TeV}$, the right-handed slepton constraint requires that $m_{\tilde{t}^{c}, 0} \sim 1.1 \mathrm{TeV}$. For $n_{10}=2, n_{5}=0$ and $M_{S} \sim 25 \mathrm{TeV}, \tilde{L}_{3}$ is driven negative and implies that $m_{\tilde{t}^{c}, 0} \sim$ $1 \mathrm{TeV}$. From Figure 6.2 it is seen that these results are comparable to the direct constraint on $m_{\tilde{t}^{c}, 0}$ obtained by requiring that color is not broken.

The positivity analysis only constrains $m_{\tilde{t}_{i}, 0} / M_{S}$ for a fixed value of $M_{3,0} / M_{S}$. To directly limit the initial scalar masses some additional information is needed. This is provided by the measured value of $\Delta m_{K}$. If some mixing and degeneracy between the first two generation scalars is assumed, parameterized by $\left(\delta_{L L}, \delta_{R R}\right)$, a minimum value for $M_{S}$ is obtained by requiring that the supersymmetric contribution to $\Delta m_{K}$ does not exceed the measured value. The results given in section 6.2 are used to calculate this minimum value. This result together with the positivity analysis then determines a minimum value for $m_{\tilde{t}^{c}, 0}$ for a given initial gluino mass $M_{3,0}$. The RG analysis is repeated with $\mu_{S}=M_{S}$, rather than $\mu_{S}=50 \mathrm{TeV}$. Only the results found by assuming GMSB mass relations between the scalars are presented. These results are shown in Figure 6.3. The mass limits for other $\tilde{f}_{i}$ are easily obtained from the information provided in Figure 6.1 and Table 6.2 and are not shown. From Figure 6.3 we find that for $\left(n_{5}, n_{10}\right)=(2,2)$ and $M_{3,0}$ less than $2 \mathrm{TeV}, m_{\tilde{t}^{c}, 0}$ must be larger than $8 \mathrm{TeV}$ for $\sqrt{\delta_{L L} \delta_{R R}}=1$, and larger than $1.8 \mathrm{TeV}$ for $\sqrt{\delta_{L L} \delta_{R R}}=0.22$. This results in $\Delta\left(m_{Z}^{2}, m_{\tilde{t}, 0}^{2}\right)$ of 2000 and 120 , respectively. In this case both the squark and selectron limits for $m_{\tilde{t}^{c}, 0}$ are comparable. The limits for other choices for $\sqrt{\delta_{L L} \delta_{R R}}$ are obtained from Figure 6.3 

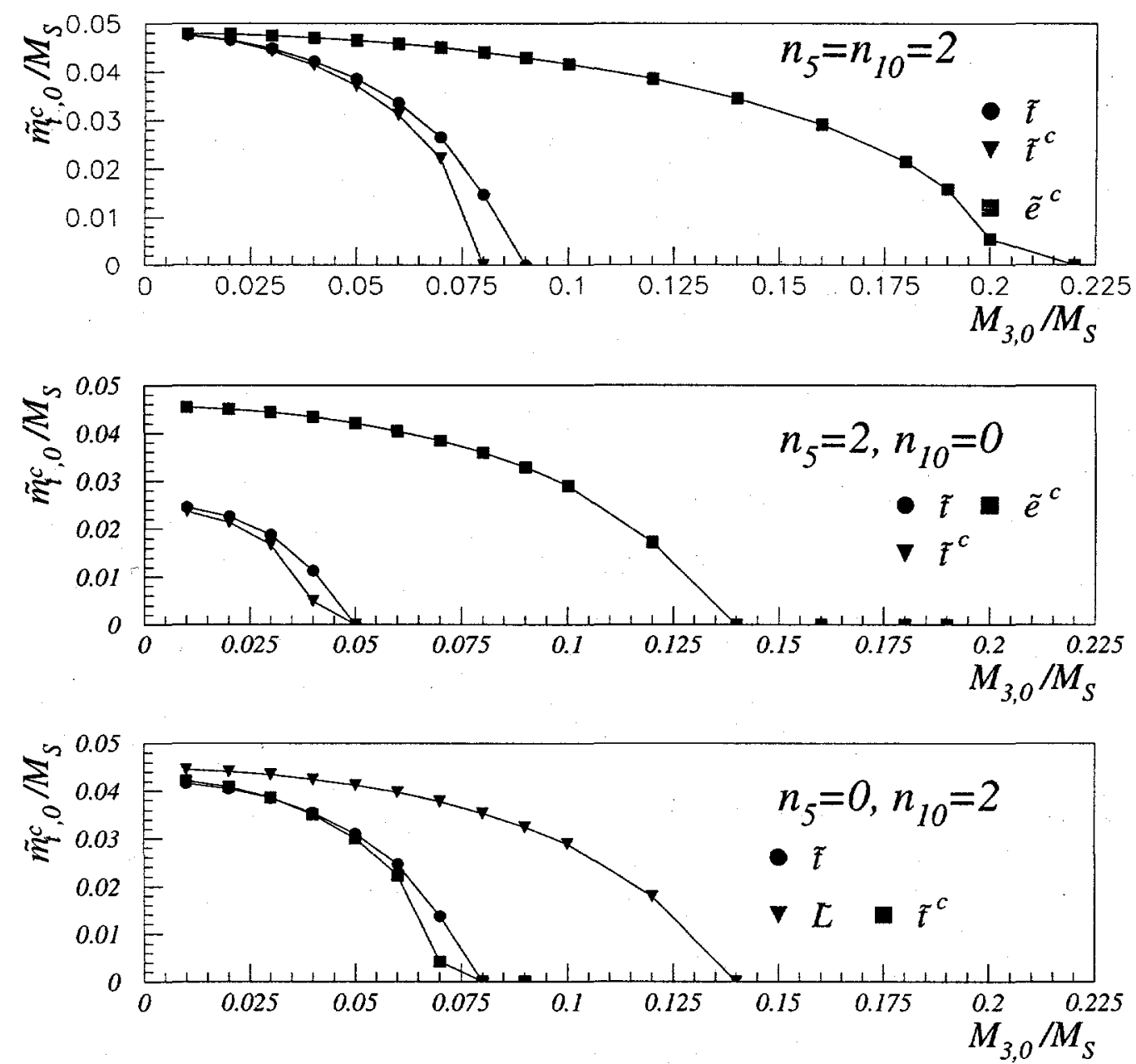

Figure 6.2: Limits for $m_{\tilde{t}^{c}, 0} / M_{S}$ from the requirement that the stop and slepton mass squared are positive at the weak scale. The regions below the curves are excluded. Low-energy gauge-mediated supersymmetry breaking mass relations between the light sparticles and $\tan \beta=2.2$ are assumed. 
by a simple scaling, since to a good approximation $\Delta m_{K} \sim \delta_{L L} \delta_{R R} / M_{S}^{2}$. For the cases $\left(n_{5}, n_{10}\right)=(2,0)$ and $(0,2)$, the corresponding limits are much weaker. In the case $\left(n_{5}, n_{10}\right)=(2,0)$, for example, only for $\delta_{R R} \sim 1$ does the selectron mass limit require that $m_{\tilde{t}^{c}, 0} \sim 1 \mathrm{TeV}$. The limits for a smaller value of $\delta$ are not shown.

This section concludes with some comments about how these results change if $C P$ violation is present in these theories with $O(1)$ phases. Recall from section 6.2 that for the same choice of input parameters, the limits on the initial stop masses increases by about a factor of 12 . This may be interpreted in one of two ways. Firstly, this constrains those models that were relatively unconstrained by the $\Delta m_{K}$ limit. Concentrate only on those models with $n_{5}=2$ and $n_{10}=0$, since this case is the most weakly constrained by the combined $\Delta m_{K}$ and positivity analysis. The conclusions for other models will be qualitatively the same. Inspecting Figure 6.3 implies that the limit $m_{\bar{t}^{c}, 0}>1 \mathrm{TeV}^{6}$ is only true if $\delta_{R R} \sim O(1)$. Smaller values of $\delta_{R R}$ do not require large initial stop masses. From the $C P$ violation constraint, however, smaller values for $\delta_{R R}$ are now constrained. For example, if $\delta_{R R} \sim 0.1$ and $O(1)$ phases are present, then $m_{\tilde{t}^{c}, 0}>1 \mathrm{TeV}$ is required. Secondly, the strong constraint from $\epsilon$ could partially or completely compensate a weakened constraint from the positivity analysis. This could occur, for example, if in an actual model the negative two-loop contribution to the stop mass squared for the same initial input parameters is smaller than the estimate used here. For example, if the estimate of the two-loop contribution in an actual model decreases by a factor of

\footnotetext{
${ }^{6}$ For GMSB relations only. The direct constraint on the stop masses is slightly weaker.
} 
$\sim(12.5)^{2}$ and $O(1)$ phases are present, the limit in this case from $\epsilon$ for the same $\delta$ is identical to the values presented in Figure 6.3.

\subsection{High Scale Supersymmetry Breaking}

This section considers the case in which SUSY breaking is communicated to the MSSM fields at a high energy scale, that is taken to be ${ }^{7} M_{G U T}=2 \times 10^{16}$ $\mathrm{GeV}$. In this case, the negative contribution of the heavy scalar soft masses to the soft mass squareds of the light scalars is enhanced by $\sim \ln \left(M_{G U T} / 50 \mathrm{TeV}\right)$, since the heavy scalar soft masses contribute to the RGE from $M_{G U T}$ to mass of the heavy scalars. It is clear that as the scale of SUSY breaking is lowered the negative contribution of the heavy scalar soft masses reduces.

This scenario was investigated in Ref.[97], and the difference between that analysis and the results presented here is briefly discussed. In the analysis of Ref.[97], the authors made the conservative choice of neglecting $\lambda_{t}$ in the RG evolution. The large value of $\lambda_{t}$ can change the analysis, and it is included here. Here it is found that for some pattern of initial stop and up-type Higgs scalar masses, e.g. universal scalar masses, this effect increases the constraint on the stop masses by almost a factor of two. This results in an increase of a factor of 3-4 in the amount of fine tuning required to obtain the correct $Z$ mass. Further,

\footnotetext{
${ }^{7}$ This choice for the high scale is done to remain agnostic about any physics appearing between the Grand Unification scale and the Planck scale. This also results in a conservative assumption, since the negative two-loop contribution is smaller with $M_{S U S Y}=M_{G U T}$.
} 

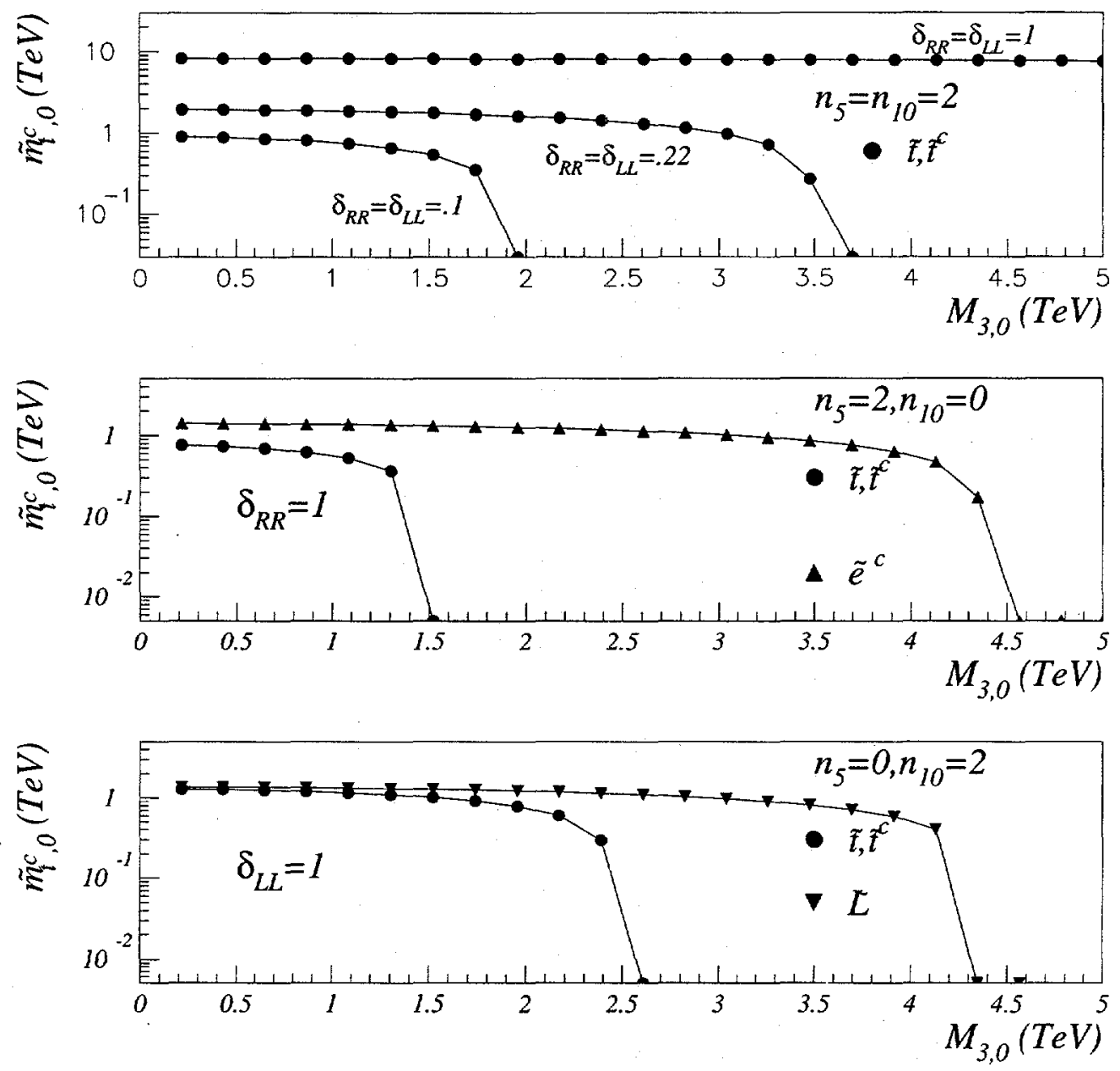

Figure 6.3: Limits for $m_{\tilde{t}^{c}, 0}$ from the requirement that the stop and slepton mass squared are positive at the weak scale while suppressing $\Delta m_{K}$, for different values of $\left(n_{5}, n_{10}\right)$, and $\left(\delta_{L L}, \delta_{R R}\right)$. The regions below the curves are excluded. Lowenergy gauge-mediated supersymmetry breaking mass relations between the light scalars and $\tan \beta=2.2$ are assumed. 
in combining the positivity analysis with the constraints from the $\Delta m_{K}$ analysis, the QCD corrections to the Flavor Changing Neutral Current (FCNC) operators has been included, as discussed in section 6.2. In the case $\left(n_{5}, n_{10}\right)=(2,2)$, this effect alone increases the positivity limit by a factor of $\sim 2-3$. The combination of these two elements imply that the positivity constraints can be quite severe.

This section proceed as follows. First, the RGEs are solved from $M_{G U T}$ to $\mu_{S}$ where the heavy scalars are decoupled. At this scale, the finite parts of the two-loop diagrams are added. Next, the RGEs are evolved (without the heavy scalar terms in the RGEs) from $\mu_{S}$ to $\mu_{G}$ using these new boundary conditions. Except where stated otherwise, the scales $\mu_{S}$ and $\mu_{G}$ are fixed to be $50 \mathrm{TeV}$ and $1 \mathrm{TeV}$, respectively.

For $\tilde{f}_{i} \neq \tilde{t}, \tilde{t}^{c}$ the numerical computations give

$$
\begin{aligned}
m_{\tilde{f}_{i}}^{2}\left(\mu_{G}\right)= & m_{\tilde{f}_{i, 0}}^{2}+\left(2.84 C_{3}^{i}+0.639 C_{2}^{i}+0.159 Y_{i}^{2}\right) M_{3,0}^{2}+c_{D} Y_{i} D_{Y, 0} \\
& -\left(4.38 C_{3}^{i}+1.92 C_{2}^{i}+0.622 Y_{i}^{2}\right) \frac{1}{2}\left(n_{5}+3 n_{10}\right) \times 10^{-3} M_{S}^{2} \\
& +\left(n_{5}-n_{10}\right)\left(17.2 M_{3,0}^{2}+\left(0.226-0.011\left(n_{5}+3 n_{10}\right)\right) M_{S}^{2}\right) Y_{i} \times 10^{-3} \\
& -0.829\left(n_{5}-n_{10}\right) Y_{i} \times 10^{-3} M_{S}^{2}
\end{aligned}
$$

These results agree with Ref.[97] for the same choice of input parameters. The term proportional to $D_{Y, 0}$, and the terms in the last line result from integrating the one-loop hypercharge $D$-term. In this case $c_{D}=-0.051$. As in the previous section, the numerical coefficients in Eqn.(6.19) depend on $\left(n_{5}, n_{10}\right)$ through the gauge coupling evolution, and the numbers in Eqn.(6.19) are for $\left(n_{5}, n_{10}\right)=(2,0)^{8}$.

\footnotetext{
${ }^{8}$ The numerical results presented in Figure 6.4 include this dependence.
} 
Requiring the soft masses squared to be positive constrains $\tilde{m}_{i, 0}^{2}=m_{i, 0}^{2}+c_{D} Y_{i} D_{Y, 0}$. Figure 6.4 plots the values of $\tilde{m}_{\tilde{f}_{i, 0}} / M_{S}$ that determine $\tilde{m}_{\tilde{f}_{i}}^{2}\left(\mu_{G}\right)=0$ as a function of $M_{3} / M_{S}$, for $\tilde{f}_{i}=\tilde{L}_{i}, \tilde{Q}_{i}, \tilde{u}_{i}^{c}, \tilde{d}_{i}^{c}$ and $\tilde{e}_{i}^{c}$. It is emphasized that the results presented in Figure 6.4 are independent of any further limits that FCNC or fine tuning considerations may imply, and are thus useful constraints on any model building attempts.

For the stops, the numerical solutions to the RGEs for $\tan \beta=2.2$ are

$$
\begin{aligned}
m_{\tilde{t}}^{2}\left(\mu_{G}\right)= & -0.021 A_{t}^{2}+0.068 A_{t} M_{3,0}+3.52 M_{3,0}^{2}+c_{D} \times \frac{1}{6} D_{Y, 0} \\
& -\bigcap .142\left(m_{H_{u}, 0}^{2}+m_{\tilde{t}^{c}, 0}^{2}\right)+0.858 m_{\tilde{t}, 0}^{2}-c_{L} \times 0.00613 M_{S}^{2} \\
m_{\tilde{t}^{c}}^{2}\left(\mu_{G}\right)= & -0.042 A_{t}^{2}+0.137 A_{t} M_{3,0}+2.33 M_{3,0}^{2}+c_{D} \times \frac{-2}{3} D_{Y, 0} \\
& -0.283\left(m_{H_{u}, 0}^{2}+m_{\tilde{t}, 0}^{2}\right)+0.716 m_{\tilde{t}^{c}, 0}^{2}-c_{R} \times 0.00252 M_{S}^{2} \\
m_{H_{u}}^{2}\left(\mu_{G}\right)= & -0.063 A_{t}^{2}+0.206 A_{t} M_{3,0}-1.72 M_{3,0}^{2}+c_{D} \times \frac{1}{2} D_{Y, 0} \\
& -0.425\left(m_{\tilde{t}, 0}^{2}+m_{\tilde{t}^{c}, 0}^{2}\right)+0.574 m_{H_{u}, 0}^{2}+c_{H} \times 0.00193 M_{S}^{2}
\end{aligned}
$$

where $\left(c_{L}, c_{R}, c_{H}\right)=(1,1,1),(3.57,4.92,5.15),(2.7,4.16,4.27)$ for $\left(n_{5}, n_{10}\right)=$ $(2,0),(2,2)$ and $(0,2)$, respectively. Also, $c_{D}=-0.051$. The mixed two-loop contribution to the $\mathrm{RG}$ evolution is $\propto\left(n_{5}-n_{10}\right)$ and is not negligible. Thus there is no simple relation between the $c$ 's for different values of $n_{5}$ and $n_{10}$. From Eqns. (6.2) and (6.1) it is clear that to keep $m_{Z}^{2}$ fine tuned at less than $1 \%$ $(\Delta \leq 100)$ in each of the bare parameters, then the following must hold : $\mu \lesssim$ $460 \mathrm{GeV} ; M_{3,0} \lesssim 300 \mathrm{GeV} ; m_{\tilde{t}_{i}, 0} \leqslant .87 \mathrm{TeV} ; m_{5, i} \leqslant 16 \mathrm{TeV}$; and $m_{10, i} \leqslant 10 \mathrm{TeV}$, for $\left(n_{5}, n_{10}\right)=(2,2)$. The fine tuning of the $Z$ mass with respect to the heavy scalars 

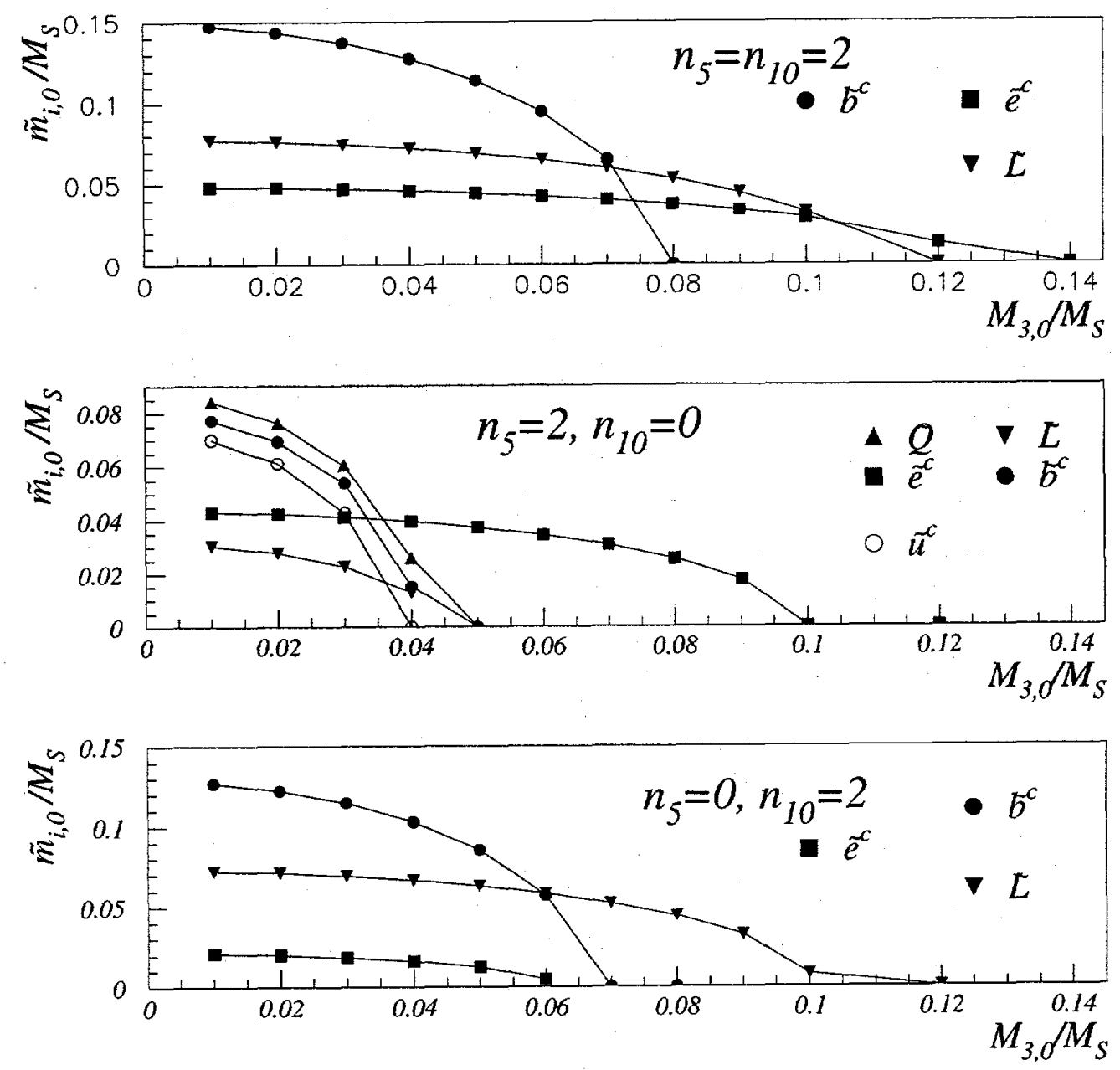

Figure 6.4: Limits for $m_{\tilde{f}_{i}, 0}$ for different values of $\left(n_{5}, n_{10}\right)$ from the requirement that the mass squareds are positive at the weak scale, assuming a supersymmetry breaking scale of $M_{G U T}$. The regions below the lines are excluded. 
is discussed in [96]. Finally, for other values of these parameters the fine tuning increases as $\Delta=100 \times \tilde{m}^{2} / \tilde{m}_{0}^{2}$, where $\tilde{m}_{0}$ is the value of $\tilde{m}$ that gives $\Delta=100$.

As in section 6.3, Eqns.(6.20) are rewritten in terms of $\tilde{m}_{i, 0}^{2}=m_{i, 0}^{2}+c_{D} Y_{i} D_{Y, 0}$. This is equivalent to setting $D_{Y, 0}=0$ in Eqns.(6.20), and relabeling $m_{i, 0}^{2} \rightarrow \tilde{m}_{i, 0}^{2}$. In what follows, the positivity analysis is used to constrain $\tilde{m}_{i, 0}^{2}$. Since $c_{D}$ is small and $D_{Y, 0} \sim O\left(m^{2}\right)$, the difference between $\tilde{m}_{i, 0}^{2}$ and $m_{i, 0}^{2}$ is small. To simplify the notation, in the remainder of this section the tilde is removed from $\tilde{m}_{i, 0}^{2}$.

As was also discussed in section 6.3 , some relations between $m_{\tilde{t}, 0}^{2}, m_{\tilde{t}^{c}, 0}^{2}$ and $m_{H_{u}, 0}^{2}$ are needed to obtain a constraint from Eqn.(6.20), using $m_{\tilde{t}}^{2}\left(\mu_{G}\right)>0$ and $m_{\tilde{t}^{c}}^{2}\left(\mu_{G}\right)>0$. Both model-dependent and model-independent constraints on the initial values of the stop masses are discussed next. The outline of the rest of this section is as follows. First, universal boundary conditions are assumed. These results are presented in Figure 6.5. Model-independent constraints are obtained by the following. Assume that $m_{H_{u}, 0}^{2}=0$ and choose $A_{t, 0}$ to maximize the value of the stop masses at the weak scale. These results are presented in Figure 6.6. It is further argued that these constraints represent minimum constraints as long as $m_{H_{u}, 0}^{2} \geq 0$. To obtain another set of model independent constraints, the electroweak symmetry breaking relation is used to eliminate $m_{H_{u}, 0}^{2}$ in favor of $\mu$. Then the positivity limits for different values of $\tilde{\mu} / M_{S}$ are presented, where $\tilde{\mu}^{2}=\mu^{2}+\frac{1}{2} m_{Z}^{2}$, and assume that $m_{H_{d}, 0}^{2}=0$ to minimize the value of $\mu$ 9. These limits are model-independent and are presented in Figure 6.7, for the

\footnotetext{
${ }^{9}$ Strictly speaking, this last assumption is unnecessary. Only the combination $\tilde{\mu}_{H}^{2} \equiv \tilde{\mu}^{2}-$
} 
case $n_{5}=n_{10}=2$. These analyses are then combined with the limits on $M_{S}$ obtained from $\Delta m_{K}$. This section then concludes with some discussion about the anomalous $D$-term solutions to the flavor problem.

First universal boundary conditions are considered for the stop and Higgs masses. That is, assume that $m_{\tilde{t}, 0}^{2}=m_{\tilde{t}^{c}, 0}^{2}=m_{H_{u}, 0}^{2}=\tilde{m}_{0}^{2}$. Figure 6.5 plots for $\tan \beta=2.2$ the minimum value of $\tilde{m}_{0} / M_{S}$ required to maintain $m_{\tilde{t}}^{2}\left(\mu_{G}\right)>0$ and $m_{\tilde{t}^{c}}^{2}\left(\mu_{G}\right)>0$. This value of $\tan \beta$ corresponds to $\lambda_{t}\left(M_{G U T}\right)=.88$, in the case that $\left(n_{5}, n_{10}\right)=(2,0)$. For comparison, the results gotten assuming $\lambda_{t}=0$ may be found in Ref.[97]. For $n_{5}=n_{10}=2$, note from figure 6.5 that if $M_{S}=$ $20 \mathrm{TeV}$ and the gaugino masses are small, the limit on the stop mass is $m_{\bar{t} c, 0} \geq$ 6.2 TeV. This limit is weakened to $6 \mathrm{TeV}$ if $M_{3,0} \lesssim 300 \mathrm{GeV}$ is allowed. Even in this case, this large initial stop mass requires a fine tuning that in this case is $\Delta \sim(6 \mathrm{TeV})^{2} / m_{Z}^{2} \sim 4200$, i.e. a fine tuning of $\$ 10^{-3}$ is needed to obtain the correct $Z$ mass.

Next assume $m_{H_{u}, 0}^{2}=0$ and choose the initial value of $A_{t, 0}$ to maximize the value of $m_{\tilde{t}_{i}}^{2}\left(\mu_{G}\right)$. The values of $m_{\tilde{t}, 0}^{2}$ and $m_{\tilde{t}^{c}, 0}^{2}$ are chosen such that $m_{\tilde{t}}^{2}\left(\mu_{G}\right)>0$ and $m_{\tilde{t}^{c}}^{2}\left(\mu_{G}\right)>0$. Note that in this case the constraint is weaker because the $\lambda_{t}$ contribution to the $\mathrm{RG}$ evolution of the stop masses is less negative. These results are plotted in Figure 6.6.

This case is discussed in some more detail to argue that the minimum value

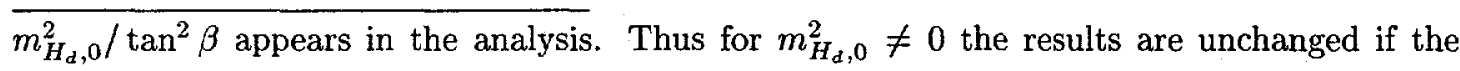
replacement $\tilde{\mu} \rightarrow \tilde{\mu}_{H}$ is made. 
of $m_{\tilde{t}_{i}, 0}$ obtained in this way will be valid for all $m_{H_{u}}^{2} \geq 0$ and all $A_{t, 0}$. Eliminate the $A_{t, 0}$ term by choosing $A_{t, 0}=k M_{3,0}$ such that the $A_{t}$ contributions to $m_{\tilde{t}_{i}}^{2}\left(\mu_{G}\right)$ is maximized. Other choices for $A_{t, 0}$ require larger values for $m_{\tilde{t}_{i}, 0}^{2}$ to maintain $m_{\tilde{t}_{i}}^{2}\left(\mu_{G}\right)=0$. The value of $k$ is determined by the following. A general expression for the value of the soft masses of the stops at the weak scale is

$$
\begin{gathered}
m_{\tilde{t}}^{2}\left(\mu_{G}\right)=-a A_{t, 0}^{2}+b A_{t, 0} M_{3,0}+c M_{3,0}^{2}+\cdots \\
m_{\tilde{t}^{c}}^{2}\left(\mu_{G}\right)=-2 a A_{t, 0}^{2}+2 b A_{t, 0} M_{3,0}+d M_{3,0}^{2}+\cdots
\end{gathered}
$$

with $a, c$ and $d$ positive. The maximum value of $m_{\tilde{t}_{i}}^{2}\left(\mu_{G}\right)$ is obtained by choosing $A_{t, 0}=b M_{3,0} / 2 a$. The value of the stops masses at this choice of $A_{t, 0}$ are

$$
\begin{aligned}
m_{\tilde{t}}^{2}\left(\mu_{G}\right) & =\left(c+\frac{b^{2}}{4 a}\right) M_{3,0}^{2}+\cdots \\
m_{\tilde{t}^{c}}^{2}\left(\mu_{G}\right) & =\left(d+2 \frac{b^{2}}{4 a}\right) M_{3,0}^{2}+\cdots
\end{aligned}
$$

An inspection of Eqn.(6.20) gives $b=0.068$ and $a=0.021$ for $\tan \beta=2.2$. In this case the "best" value for $A_{t, 0}$ is $A_{t, 0}^{B} \sim 1.6 M_{3,0}$. It then follows that the quantity $b^{2} / 4 a=0.055$ is a small correction to the coefficient of the gaugino contribution in Eqn.(6.20). Thus the difference between the minimum initial stop masses for $A_{t, 0}=0$ and $A_{t, 0}=A_{t, 0}^{B}$ is small. Next assume that $m_{H_{u}, 0}^{2}=0$. Requiring that both $m_{\tilde{t}}^{2}\left(\mu_{G}\right)=0$ and $m_{\tilde{t}^{c}}^{2}\left(\mu_{G}\right)=0$ determines a minimum value for $m_{\tilde{t}, 0}^{2}$ and $m_{\tilde{t}^{c}, 0}^{2}$. Now since the $m_{H_{u}, 0}^{2}$ contribution to both the stop soft masses is negative (see Eqn.(6.20)), the minimum values for $m_{\tilde{t}_{i}, 0}^{2}$ found by the preceding procedure are also minimum values if any $m_{H_{u}, 0}^{2}>0$ is allowed. 
So for all $A_{t, 0}$ and all $m_{H_{u}, 0}^{2} \geq 0$, the limits presented in Figure 6.6 represent lower limits on the initial stop masses if it is required that the soft masses remain positive at the weak scale. Further, the limits in this case are quite strong. For example, Figure 6.6 implies that if $M_{S} \sim 20 \mathrm{TeV}$ and $M_{3,0} \sim 200 \mathrm{GeV}$ (so that $M_{3,0} / M_{S} \sim 10^{-2}$ ), then the initial stop masses must be greater than $3.5 \mathrm{TeV}$ in the case that $\left(n_{5}, n_{10}\right)=(2,2)$. The results are stronger in a more realistic scenario, i.e. $m_{H_{u}, 0}^{2}>0$. If, for example, $m_{H_{u}, 0}^{2} \sim m_{t^{c}, 0}^{2} / 9$ the constraints are larger by only a few percent. In the case that $m_{H_{u}, 0}^{2}=m_{\tilde{t}^{c}, 0}^{2}=m_{\tilde{t}, 0}^{2}$, presented in Figure 6.5, however, the constraint on the initial $\tilde{t}^{c}$ mass increases by almost a factor of two.

So far relation between $m_{H_{u}, 0}^{2}$ and $m_{t^{c}, 0}^{2}$ has been assumed in order to obtain constraints on the initial stop masses; e.g., $m_{H_{u}, 0}^{2}=0$ or $m_{H_{u}, 0}^{2}=m_{t^{c}, 0}^{2}$. Perhaps a better approach is to use the EWSB relation, Eqn.(6.2), to eliminate $m_{H_{u}, 0}^{2}$ in favor of $\mu^{2}$. This has the advantage of being model-independent. It is also a useful reorganization of independent parameters since the amount of fine tuning required to obtain the correct $Z$ mass increases as $\mu$ is increased. To obtain some limits $m_{H_{d}, 0}^{2}=0$ is chosen ${ }^{10}$ to minimize the value of $\mu^{2}$, and $m_{H_{u}, 0}^{2}$ is required to be positive. The minimum value of $m_{\tilde{t}^{c}, 0} / M_{S}$ and $m_{\tilde{t}, 0} / M_{S}$ for different choices of $\tilde{\mu} / M_{S}$ are gotten by solving $m_{\tilde{t}^{c}}^{2}\left(\mu_{G}\right)=0$ and $m_{\tilde{t}}^{2}\left(\mu_{G}\right)=0$. These results are presented in figure 6.7. In this figure the positivity constraints terminate at that value of $M_{3,0}$ which gives $m_{H_{u}, 0}^{2}=0$.

As discussed in the above, reducing the value of $m_{H_{u}, 0}^{2}$ decreases the positivity

${ }^{10}$ This assumption is unnecessary. See the previous footnote. 
limit on $m_{\tilde{t}_{i}, 0}$. Consequently the fine tuning of $m_{Z}^{2}$ with respect to $m_{\tilde{t}_{i}, 0}^{2}$ is also reduced. But using Eqns.(6.20) and (6.2), it can be seen that decreasing $m_{H_{u}, 0}^{2}$ while keeping $m_{\tilde{t}^{c}}^{2}\left(\mu_{G}\right)=0$ and $m_{\tilde{t}}^{2}\left(\mu_{G}\right)=0$ results in a larger $\mu$, thus increasing the fine tuning with respect to $\mu$. This can also be seen from figure 6.7. It is found, for example, that if $M_{3,0} / M_{S} \sim 0.01$, the small value $\tilde{\mu} / M_{S}=0.01$ requires $m_{\bar{t}_{i}, 0} / M_{S} \sim 0.25$. For $M_{S}=10 \mathrm{TeV}$, this corresponds to $\mu \sim 100 \mathrm{GeV}$ and $m_{\tilde{t}_{i}, 0} \geq 2.5 \mathrm{TeV}$. A further inspection of figure 6.7 shows that for the same value of $M_{3,0} / M_{S}$, a value of $m_{\tilde{t}, 0} / M_{S}=0.17$ is allowed (gotten by decreasing $m_{H_{u}, 0}^{2}$ ) only if $\tilde{\mu} / M_{S}$ is increased to 0.14 . This corresponds to $\mu=1.4 \mathrm{TeV}$ for $M_{S}=10$ $\mathrm{TeV}$; this implies that $\Delta\left(m_{Z}^{2} ; \mu\right) \sim 930$. Thus the limit on the initial stop masses can only be decreased at the expense of increasing $\mu$.

Finally, the limits become weaker if $m_{H_{u}, 0}^{2}<0$. This possibility is theoretically unattractive on two accounts. Firstly, a nice feature of supersymmetric extensions to the SM is that the dynamics of the model, through the presence of the large top quark Yukawa coupling, naturally leads to the breaking of the electroweak symmetry[86]. This is lost if electroweak symmetry breaking is already present at the tree-level. Secondly, the fine tuning required to obtain the correct $Z$ mass is increased. Figure 6.7 implies that while reducing $m_{H_{u}, 0}^{2}$ below zero does reduce the limit on the initial stop masses, the value of $\mu$ increases beyond the values quoted in the previous paragraph, thus further increasing the fine tuning of the $Z$ mass. This scenario is not discussed any further.

Next the positivity analysis of this section is combined with the results of 

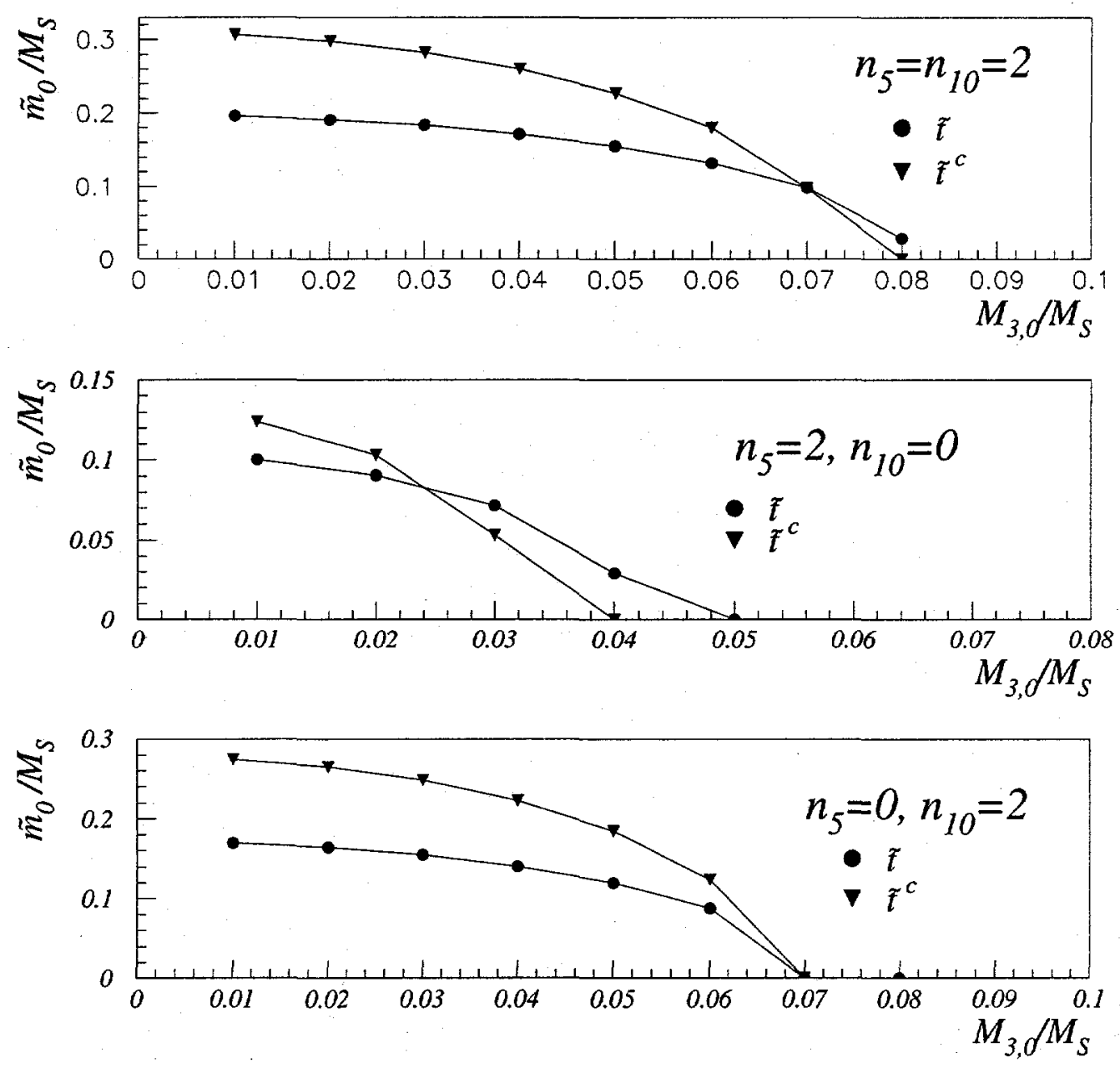

Figure 6.5: Limits for $\tilde{m}_{0} / M_{S}$ from the requirement that the stop masses squared are positive at the weak scale, for $\tan \beta=2.2, A_{t, 0}=0$ and assuming universal scalar masses at $M_{G U T}$ for the stop and Higgs scalars. The regions below the curves are excluded. 

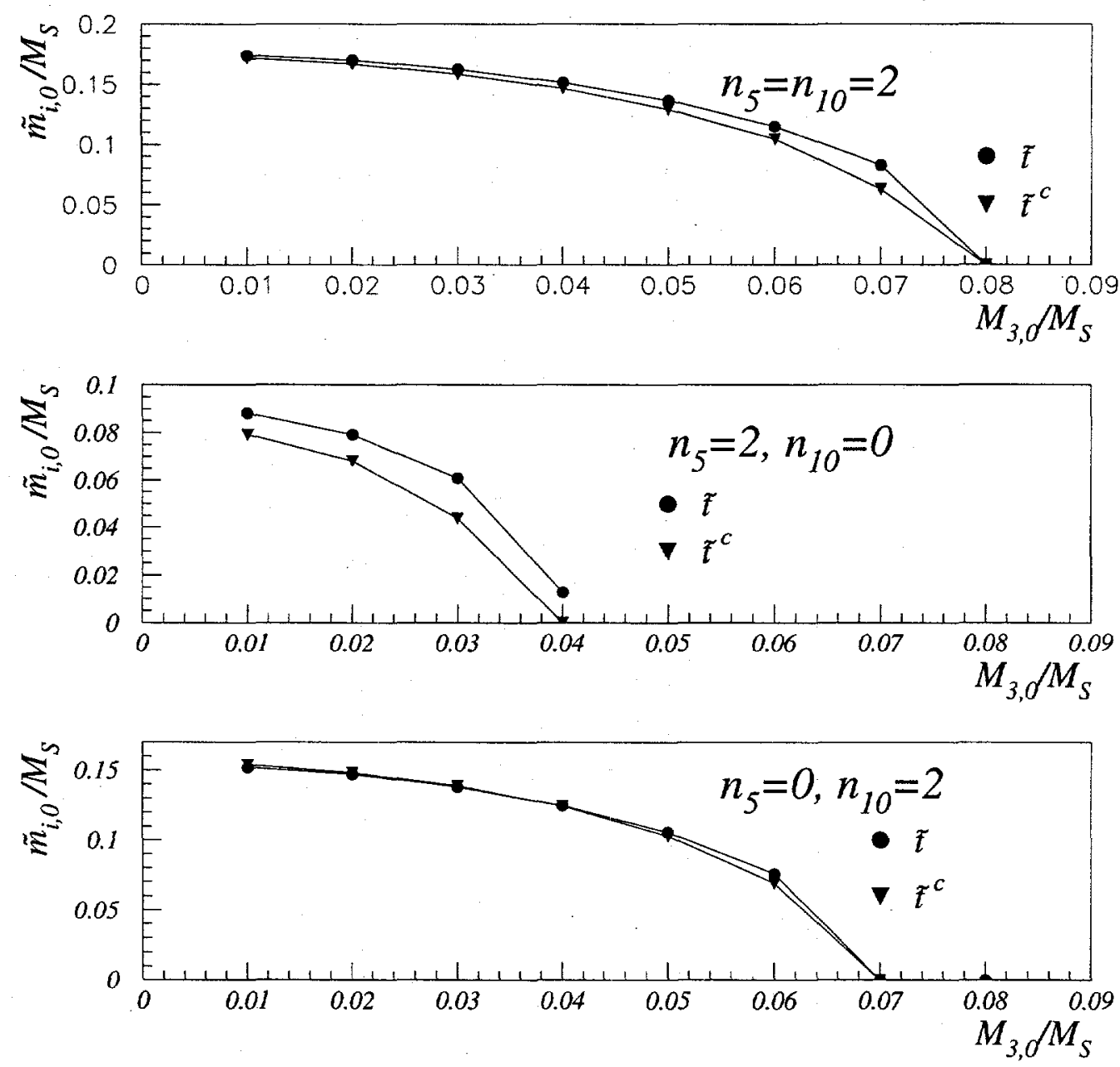

Figure 6.6: Limits for $m_{\bar{t}, 0} / M_{S}, m_{\tilde{t}^{c}, 0} / M_{S}$, from the requirement that the stop masses squared are positive at the weak scale, for $M_{S U S Y}=M_{G U T}, \tan \beta=2.2$ and assuming that $m_{H_{u}, 0}^{2}=0$. The value of $A_{t, 0}$ is chosen to maximize the value of the stop soft masses at the weak scale. The regions below the curves are excluded. 


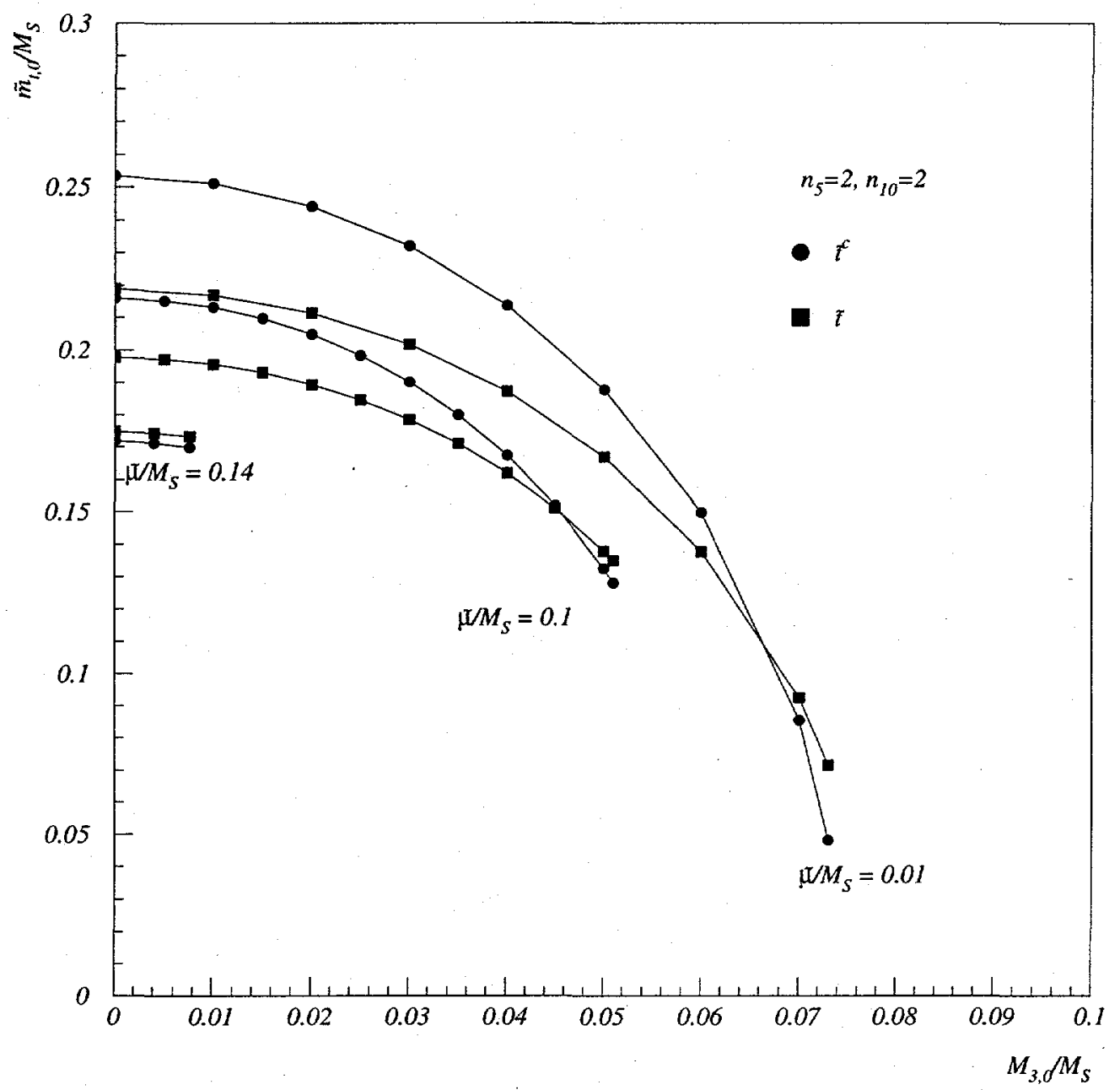

Figure 6.7: Limits for $m_{\tilde{t}, 0} / M_{S}, m_{\tilde{t} c}, 0 / M_{S}$, from the requirement that the stop masses squared are positive at the weak scale, for $\left(n_{5}, n_{10}\right)=(2,2), M_{S U S Y}=$ $M_{G U T}, \tan \beta=2.2$, and different values of $\tilde{\mu} / M_{S}$. The contours end at that value of $M_{3,0} / M_{S}$ that gives $m_{H_{u}, 0} / M_{S}=0$. The value of $A_{t, 0}$ is chosen to maximize the value of the stop soft masses at the weak scale. The regions below the lines are excluded. 
section 6.2 to place lower limits on the soft scalar masses. For given values of $\delta_{L L}, \delta_{R R}$, a minimum value of $M_{S}, M_{S, m i n}$, is found using the results of section 6.2 . This is combined with the positivity analysis in Figure 6.6, to produce the results shown in Figure 6.8. Also shown are other limits gotten by assuming $m_{H_{u}, 0}^{2}=m_{\tilde{t}^{c}, 0}^{2}$. These results are presented in Figure 6.9. Figure 6.10 also presents the stop mass limits for different values of $\mu$, restricted to $m_{H_{u}, 0}^{2} \geq 0$ and for $\sqrt{\delta_{L L} \delta_{R R}}=0.04$. In all cases the heavy scalars were decoupled at $M_{S, \min }$, rather than $50 \mathrm{TeV}$, and so the positivity analysis was repeated. The value of $A_{t, 0}$ was chosen to maximize the value of the stop masses at the weak scale. For completeness, the results for the cases $\left(n_{5}, n_{10}\right)=(2,0)$ and $(0,2)$ and $m_{H_{u}, 0}^{2}=0$ are presented in Figure 6.11. To repeat: the minimum allowable values for the stop masses consistent with $m_{H_{u}, 0}^{2}>0$, gotten by setting $m_{H_{u}, 0}^{2}=0$, are given in Figures 6.8 and 6.11 .

Next some consequences of this numerical analysis are discussed. Only the case $n_{5}=n_{10}=2$ is considered, since this is the relevant case to consider if the supersymmetric flavor problem is explained by decoupling the heavy scalars. Other choices for $n_{5}$ and $n_{10}$ require additional physics to explain the required degeneracy or alignment of any light non-third generation scalars. From Figures 6.8 and 6.9 it is seen that for $\sqrt{\delta_{L L} \delta_{R R}}=0.22$ and $M_{3,0} \leq 1 \mathrm{TeV}, m_{\tilde{t}_{i}, 0} \gtrsim 7 \mathrm{TeV}$ is required. If instead both $\Delta\left(m_{Z}^{2} ; M_{S}^{2}\right)$ and $\Delta\left(m_{Z}^{2} ; M_{3,0}\right)$ are restricted to be less than 100 , then $M_{S} \lesssim 10 \mathrm{TeV}$ and $M_{3,0} \lesssim 300 \mathrm{GeV}$ is required. To not be excluded by $\Delta m_{K}$, further require that $\sqrt{\delta_{L L} \delta_{R R}} \lesssim 0.06$. For this value of $\sqrt{\delta_{L L} \delta_{R R}}=0.06$, a minimum value for $m_{\tilde{t}, 0}$ of $\sim 1.5-2.5 \mathrm{TeV}$ is gotten by rescaling the results in 
figures 6.8 and 6.9 for $\sqrt{\delta_{L L} \delta_{R R}}=0.04$ by an amount $0.06 / 0.04$. The range depends on the value of $m_{H_{u}, 0}^{2}$, with the lower (upper) limit corresponding to $m_{H_{u}, 0}^{2}=0$ $\left(m_{\tilde{t}^{c}, 0}^{2}\right)$. Thus $\Delta\left(m_{Z}^{2} ; m_{\tilde{t}_{i}, 0}^{2}\right) \sim 400-800$. This fine tuning can be reduced only by either increasing $M_{3,0}$ - which increases $\Delta\left(m_{Z}^{2}, M_{3,0}\right)$ beyond 100 - or by reducing $M_{S}$ - which requires a smaller value for $\sqrt{\delta_{L L} \delta_{R R}}$. So unless $\sqrt{\delta_{R R} \delta_{L L}}$ is naturally small, decoupling the heavy scalars does not provide a natural solution to the flavor problem.

This section concludes by discussing the constraint this analysis implies for those models which generate a split mass spectrum between different generations through the $D$-term contributions of the anomalous $U(1)$ gauge symmetry[91, 95 , 94]. In the model of set $\mathrm{D}$ of [94], there are two $\overline{5} \mathrm{~s}$ at $7 \mathrm{TeV}$ and $6.1 \mathrm{TeV}$ and two $10 \mathrm{~s}$ at 6.1 and $4.9 \mathrm{TeV}$, respectively, so that $\Delta m_{K}$ is suppressed. These values must be increased by a factor of 2.5 to correct for the QCD enhancement of the SUSY contribution to $\Delta m_{K}$, as discussed in section 6.2. To obtain a conservative bound on the initial stop masses from the positivity requirement, first assume that all the heavy scalars have a common mass $M_{S}=2.5 \times 5 \mathrm{TeV}=12.5 \mathrm{TeV}$. (It would have been $5 \mathrm{TeV}$ without the QCD correction.) Then assuming a weak scale value of the gluino mass that is less than $710 \mathrm{GeV}$ and setting $m_{H_{u}, 0}^{2}=0$ $\left(m_{\tilde{t}^{c}, 0}^{2}\right)$, Figure $6.6(6.5)$ implies that $m_{\tilde{t}, 0} \geq 2.1(3.6) \mathrm{TeV}$ is required. This leads to $\Delta\left(m_{Z}^{2} ; m_{\tilde{t}, 0}^{2}\right) \geq 580(1700)$. To obtain a better bound, the analysis is repeated using $n_{5} m_{5}^{2}+3 n_{10} m_{10}^{2}=\left((7 \mathrm{TeV})^{2}+(6.1 \mathrm{TeV})^{2}+3 \times(6.1 \mathrm{TeV})^{2}+3 \times(4.9 \mathrm{TeV})^{2}\right) \times(2.5)^{2}$ It is possible to do this since only this combination appears in the RG analysis 

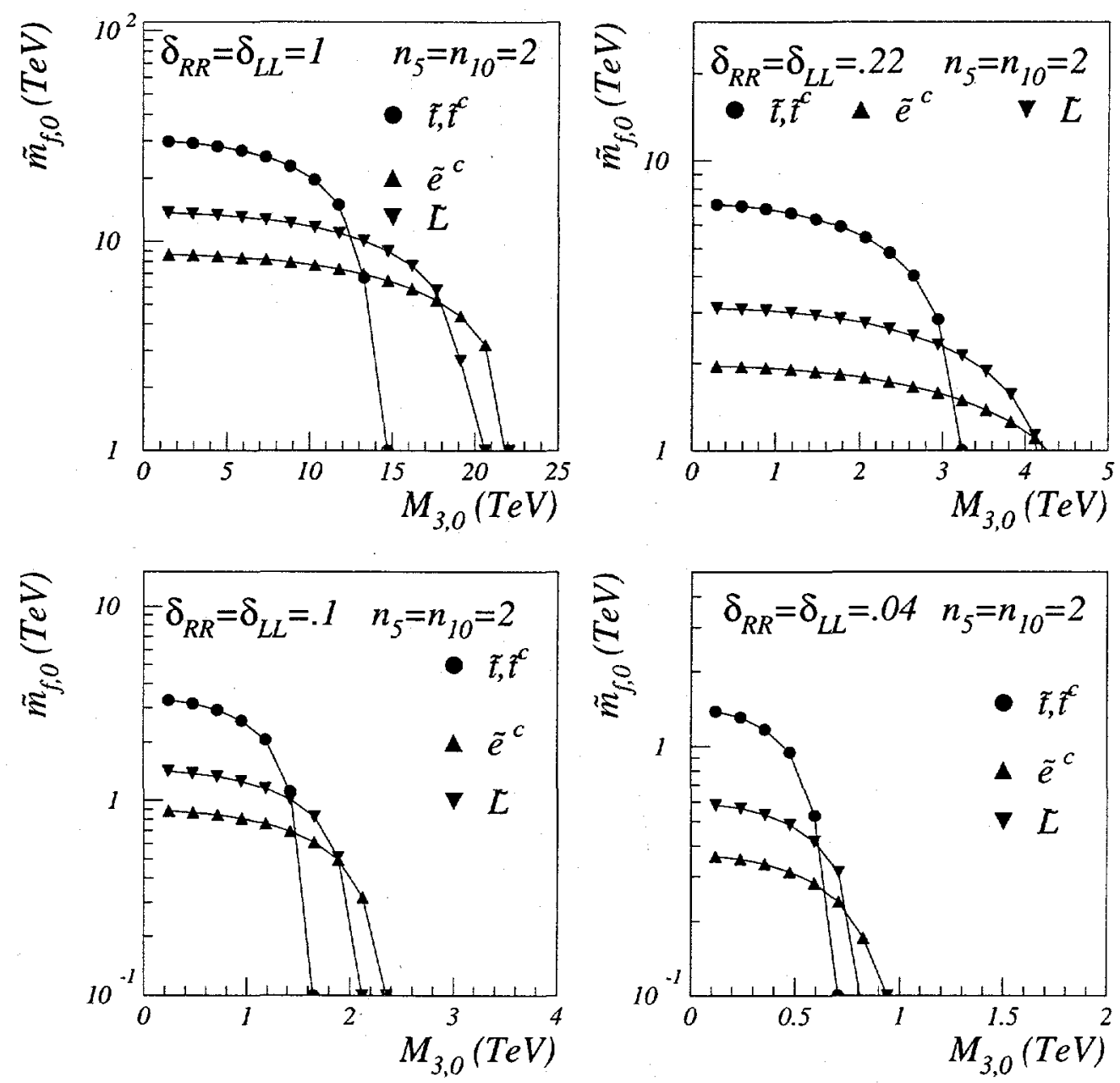

Figure 6.8: Limits for $m_{\tilde{t}, 0}$ and $m_{\tilde{t}^{c}, 0}, m_{\tilde{e}}$, and $m_{\tilde{L}}$ from the requirement that the masses squared are positive at the weak scale while suppressing $\Delta m_{K}$. It was assumed that $M_{S U S Y}=M_{G U T}, \tan \beta=2.2$ and that $m_{H_{u}, 0}^{2}=0$. The value of $A_{t, 0}$ was chosen to maximize the value of the stop soft masses at the weak scale. The heavy scalars were decoupled at the minimum value allowed by $\Delta m_{K}$. The regions below the lines are excluded. 

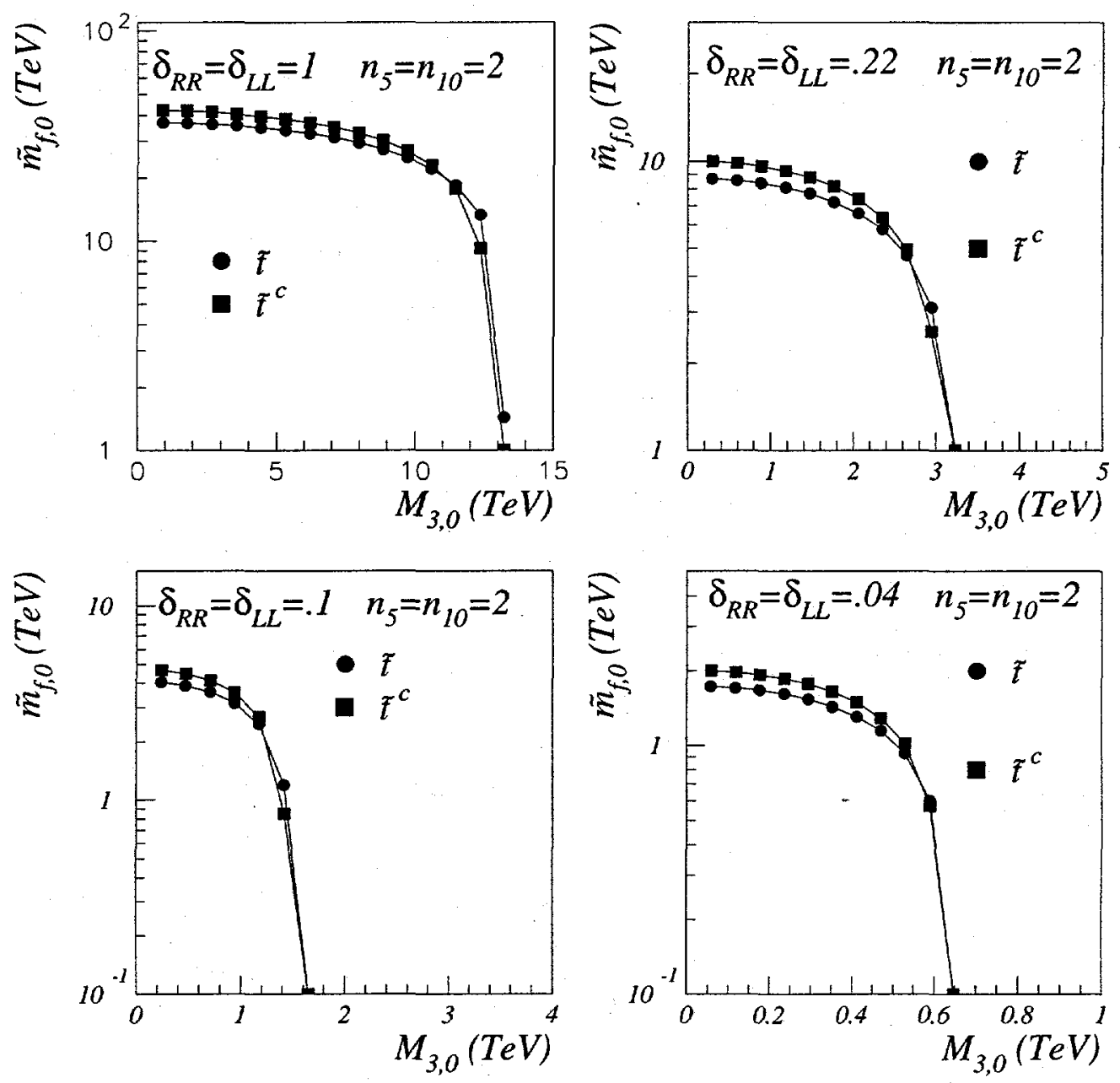

Figure 6.9: Limits for $m_{\tilde{t}, 0}$ and $m_{\tilde{t}^{c}, 0}$ from the requirement that the stop masses squared are positive at the weak scale while suppressing $\Delta m_{K}$. It was assumed that $M_{S U S Y}=M_{G U T}, \tan \beta=2.2$ and that $m_{H_{u}, 0}^{2}=m_{\tilde{t} c, 0}^{2}$. The value of $A_{t, 0}$ was chosen to maximize the value of the stop soft masses at the weak scale. The heavy scalars were decoupled at the minimum value allowed by $\Delta m_{K}$. The regions below the lines are excluded. 


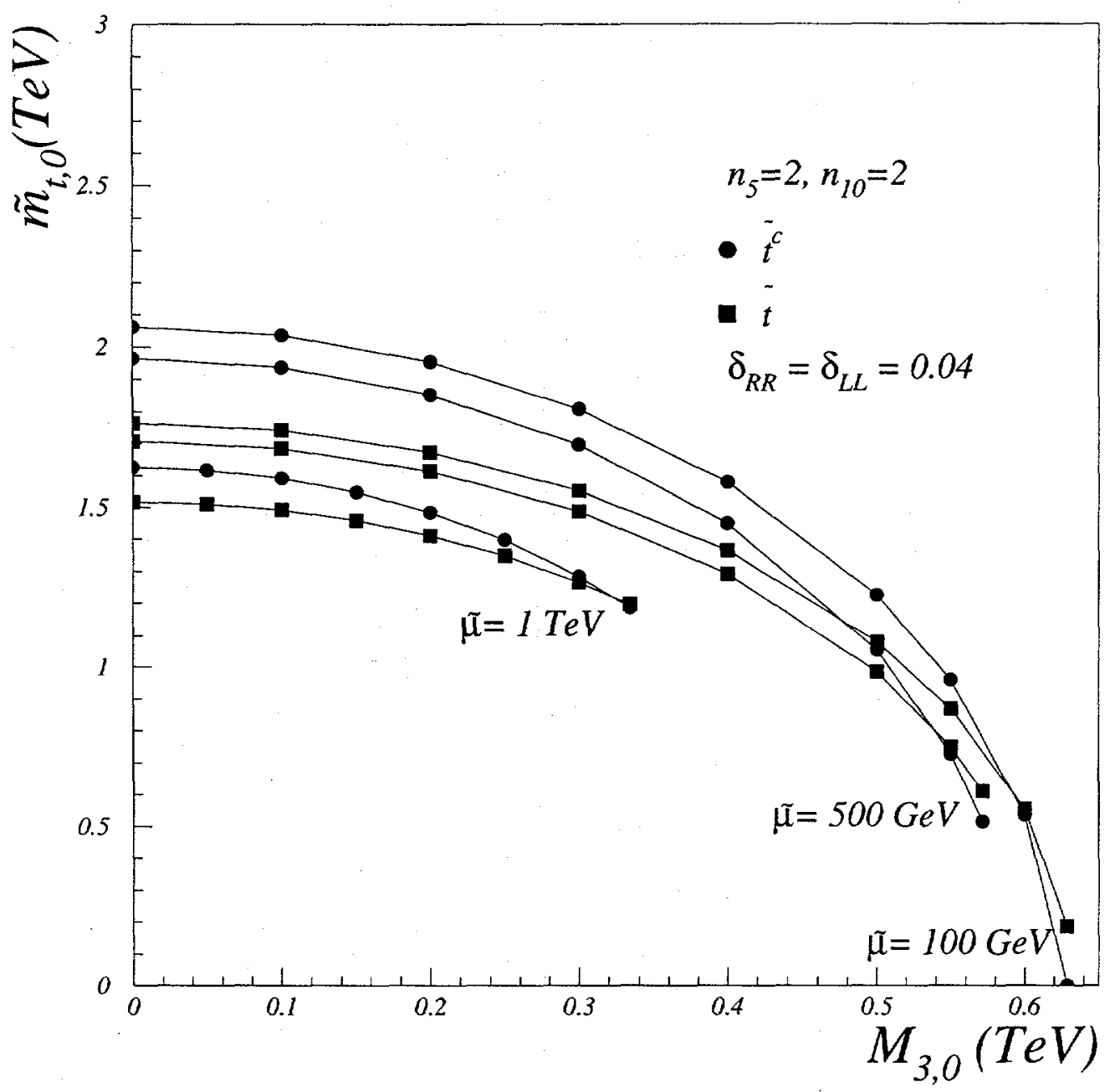

Figure 6.10: Limits for $m_{\tilde{t}, 0}$ and $m_{\tilde{t}^{c}, 0}$ from the requirement that the stop masses squared are positive at the weak scale while suppressing $\Delta m_{K}$, for $\left(n_{5}, n_{10}\right)=$ $(2,2), \sqrt{\delta_{L L} \delta_{R R}}=0.04$, and different values of $\mu$. The contours terminate at $m_{H_{u}, 0}^{2}=0$. It was assumed that $M_{S U S Y}=M_{G U T}$ and $\tan \beta=2.2$. The value of $A_{t, 0}$ was chosen to maximize the value of the stop soft masses at the weak scale. The heavy scalars were decoupled at the minimum value allowed by $\Delta m_{K}$. The regions below the lines are excluded. 

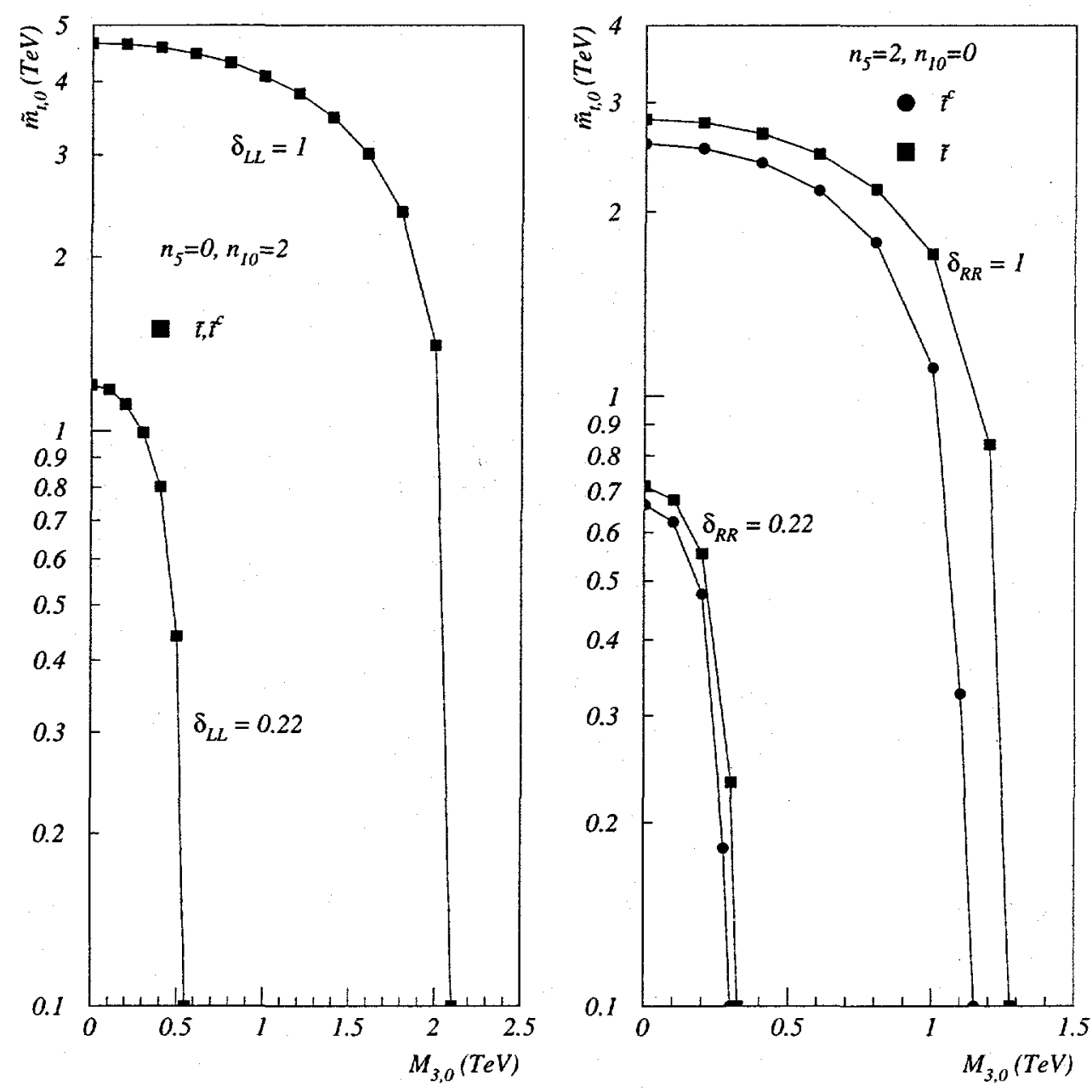

Figure 6.11: Limits for $m_{\bar{t}, 0}, m_{\tilde{t}^{c}, 0}$ from the requirement thit the stop masses squared are positive at the weak scale while suppressing $\Delta m_{K}$, for the cases $\left(n_{5}, n_{10}\right)=(2,0)$ and $(0,2)$. It was assumed that $M_{S U S Y}=M_{G U T}, \tan \beta=2.2$ and that $m_{H_{u}, 0}^{2}=0$. The value of $A_{t, 0}$ was chosen to maximize the value of the stop soft masses at the weak scale. The heavy scalars were decoupled at the minimum value allowed by $\Delta m_{K}$. The regions below the lines are excluded. 
for $\left(n_{5}, n_{10}\right)=(2,2)$. It is found (assuming $m_{H_{u}, 0}^{2}=0$ and the gluino mass at the weak scale is less than $710 \mathrm{GeV}$ ) that $m_{\tilde{t}, 0} \gtrsim 2.4 \mathrm{TeV}$. In the model of [95], $\delta_{R R} \approx \delta_{L L} \approx 0.01$. To obtain a limit on the initial stop masses, use the bound obtained from either Figures 6.8 or 6.9 for $\delta_{R R}=\delta_{L L} \approx 0.04$, and divide the limit by a factor of 4 . By inspecting these Figures it is seen that this model is only weakly constrained, even if $m_{H_{u}, 0}^{2} \sim m_{\tilde{t}, 0}^{2}$. Next the limits in this model when $O(1) C P$ violating phases are present is discussed. To obtain the minimum value of $M_{S}$ in this case, the minimum value of $M_{S}$ obtained from the $\Delta m_{K}$ constraint for $\delta_{L L}=\delta_{R R}=0.04$ should be multiplied by $12.5 / 4$; dividing by 4 gives the result for $\delta_{L L}=\delta_{R R}=0.01$, and multiplying by 12.5 gives the constraint on $M_{S}$ from $\epsilon$. The result is $M_{S} \gtrsim 23 \mathrm{TeV}$. Next, assume that $M_{3,0}$ is less than $300 \mathrm{GeV}$, so that the value of the gluino mass at the weak scale is less than $710 \mathrm{GeV}$. This gives $M_{3,0} / M_{S} \leq 0.013$. Using these values of $M_{3,0}$ and $M_{S}$, an inspection of Figures 6.5 and 6.6 implies that $m_{\tilde{t}, 0}$ must be larger than $3.9 \mathrm{TeV}$ to $6.9 \mathrm{TeV}$, depending on the value of $m_{H_{u}, 0}^{2}$. This gives $\Delta\left(m_{Z}^{2} ; m_{\tilde{t}, 0}^{2}\right) \geq 2000$. In the model of [91], $M_{3,0} / M_{S} \approx 0.01$ and $m_{\tilde{f}, 0} / M_{S} \approx 0.1$. Inspecting Figures 6.5 and 6.6 implies that these values are excluded for $\left(n_{5}, n_{10}\right)=(2,2)$ and $(0,2)$. The case $(2,0)$ is marginally allowed. The model of [91] with $\left(n_{5}, n_{10}\right)=(2,2)$ and $\lambda_{t}=0$ was also excluded by the analysis of Ref.[97].

\subsection{Using Finetuning to Constrain $\delta$}

In this section, the messenger scale, $M_{S U S Y}$, is varied between the GUT scale 
and a low scale $\sim 50 \mathrm{TeV}$, and the boundary values of the stop and gluino masses are restricted so that EWSB is not fine tuned. This gives us an upper limit to $\delta$ if both positivity of the stop mass squareds at the weak scale and suppression of $\Delta m_{K}$ is imposed. In other words, values for $\left(\delta, M_{S U S Y}\right)$ are determined which satisfy the following requirements: 1 . Suppression of the SUSY contribution to $\Delta m_{K}$ by making the mass of the first two generation scalars, $M_{S}$, large. 2. Positivity of the stop masses squared and 3. Fine tuning in electroweak symmetry breaking does not exceed $1 \%$ or $10 \%$ (i.e., both $\Delta\left(m_{Z}^{2}, m_{\tilde{t}, 0}^{2}\right)$ and $\Delta\left(m_{Z}^{2}, M_{3,0}\right)$ are smaller than either 100 or 10 ).

An upper limit to $\delta$ satisfying the above requirements is obtained as follows. For a given $M_{S U S Y}$ compute, using Eqns.(6.1) and (6.2), the boundary values of the stop mass, $m_{\bar{t}, \max }$, and the gluino mass, $M_{3, \max }$, such that both $\Delta\left(m_{Z}^{2}, m_{\tilde{t}, 0}^{2}\right)$ and $\Delta\left(m_{Z}^{2}, M_{3,0}\right)$ are equal to some maximum value $\Delta_{\max }$ which is chosen to be 100 or 10 . $^{11}$ Substituting these values of the bare stop ${ }^{12}$ and gluino masses into

\footnotetext{
${ }^{11}$ In computing the $\Delta$ 's, $\tan \beta$, in addition to $m_{H_{u}}^{2}\left(m_{Z}\right)$, should be regarded as a function of the bare parameters. However, this additional contribution to the $\Delta$ 's is small for $\tan \beta \gtrsim 2$ and also makes the magnitude of $\Delta$ larger. This dependence which is a conservative choice is neglected.

${ }^{12}$ Strictly speaking, we should translate the upper bound on $m_{\tilde{t}_{i}, 0}^{2}$ into an upper bound on $\tilde{m}_{\tilde{t}_{i}, 0}^{2}$ using $\tilde{m}_{\tilde{t}_{i}, 0}^{2}=m_{\tilde{t}_{i}, 0}^{2}+c_{D} Y_{\tilde{t}_{i}} D_{Y, 0}+Y_{\tilde{t}_{i}} \zeta_{D}$, i.e., to that combination appearing in the positivity constraint. Instead, we use the same bound for both $m_{\tilde{t}_{i}, 0}^{2}$ and $\tilde{m}_{\tilde{t}_{i}, 0}^{2}$. This is reasonable, since $c_{D}$ is generally small $(\lessgtr 0.05)$, and $D_{Y, 0} \sim O\left(m^{2}\right)$. In any case, this effect is in the opposite direction for $\tilde{t}$ and $\tilde{t}^{c}$. In the case that $\zeta_{D} \neq 0$, a slightly larger $(O(30 \%))$ value for $\delta$ may be allowed as compared to $\zeta_{D}=0$. This is because if $\zeta_{D}<0$, the maximum value
} 
the expression for the weak-scale value of the stop mass squared, determines the maximum value of $M_{S}, M_{S, m a x}$, such that the stop masses squared at the weak scale are positive. Using this value for $M_{S}$ and the analysis described in section 6.2.1, an upper limit to $\delta$ is obtained from the $\Delta m_{K}$ constraint. This value of $\delta$ and $M_{S U S Y}$ then satisfies the above-mentioned three requirements. This can be seen as follows. For the given $M_{S U S Y}$, if $\delta$ is larger than this limit, then to suppress $\Delta m_{K}, M_{S}$ has to be larger than $M_{S, m a x}$. But, then to keep the stop masses squared positive at the weak scale, the boundary value of either the stop or the gluino mass has to increase beyond $m_{\tilde{t}, \max }$ or $M_{3, \max }$ respectively, leading to $\Delta\left(m_{Z}^{2}, m_{\tilde{t}, 0}^{2}\right)$ or $\Delta\left(m_{Z}^{2}, M_{3,0}\right)$ larger than $\Delta_{m a x}$, i.e., increasing the fine tuning in EWSB.

In Figures 6.12 are shown the limits on $\sqrt{\delta_{L L} \delta_{R R}}$ as a function of $M_{S U S Y}$ for the case $\left(n_{5}=2, n_{10}=2\right)$. In the top of figure $6.12, m_{H_{u}, 0}^{2}=0$ is assumed. GMSB relations between the stop and Higgs masses are assumed in the bottom of Figure

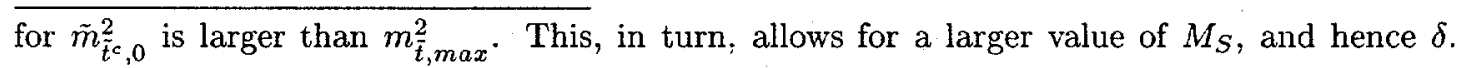
Naturalness considerations limit $\left|\zeta_{D}\right|$, though. The EWSB relation for $m_{Z}^{2}$, Eqn.(6.2), contains a term linear in $\zeta_{D}$. Requiring that $\Delta\left(m_{Z}^{2}, \zeta_{D}\right)<100$ implies that $\left|\zeta_{D}\right| \leqslant \zeta_{D, \max } \equiv(900 \mathrm{GeV})^{2}$. Thus for a high scale of supersymmetry breaking, the upper bound on $\tilde{m}_{\tilde{t}^{c}, 0}^{2}$ may be increased to $\tilde{m}_{\tilde{t}^{c}, 0}^{2} \sim m_{\tilde{t}, \text { max }}^{2}+\frac{2}{3} \zeta_{D, \text { max }} \sim \frac{5}{3} m_{\tilde{t}_{\text {, max }}}^{2}$, while maintaining $\Delta\left(m_{Z}^{2}, m_{\tilde{t}^{c}, 0}^{2}\right)=\Delta\left(m_{Z}^{2}, \zeta_{D}\right)=100$. This roughly translates into an increase of $\sim \sqrt{5 / 3}=1.3$ in the limit to $\delta$. The actual limit will be smaller, since with this choice of sign for $\zeta_{D}$, the positivity constraint for the left-handed stop is now stronger. It is thus reasonable to require that the maximum value of $\tilde{m}_{\tilde{t}_{i}, 0}^{2}$ be comparable to $m_{\tilde{t}_{i}, \max }^{2}$. 
6.12. For both cases, $\Delta_{\max }=100, \tan \beta=2.2$ and 10 are considered. For other choices for $\Delta_{\max }$, the upper limit to $\delta$ roughly scales as $\sqrt{\Delta_{\max } / 100}$, since both $m_{\tilde{t}, \max }, M_{3, \max }$ and therefore $M_{S, \max }$ scale as $\sqrt{\Delta_{\max }}$.

In the case of GMSB mass relations, the boundary value of the Higgs mass and the stop masses are comparable for high $M_{S U S Y}$. Since $m_{H_{u}, 0}^{2}$ results in a negative contribution to the stop mass squared, this tends to reduce the stop mass squared at the weak scale as compared to the case $m_{H_{u}, 0}^{2}=0$. Then, from the above analysis, it is possible to see that $M_{S, \max }$ and, in turn, the limit on $\delta$ is smaller for the GMSB case as compared to the case $m_{H_{u}, 0}^{2}=0$. This can be seen by comparing the top and bottom of Figure 6.12.

In Figure 6.13 the limits on $\delta_{R R}$ and $\delta_{L L}$ for $\left(n_{5}=2, n_{10}=0\right)$ and $\left(n_{5}=\right.$ $\left.0, n_{10}=2\right)$ are shown, respectively. Here $m_{H_{u}, 0}^{2}=0$ is assumed and $\tan \beta=2.2$ and 10 are considered. If $\Delta_{\max }$ is chosen to be 100 , then a constraint on $\delta(\delta \lesssim 0.5)$ is obtained only for high values of $M_{S U S Y}$. So, instead $\Delta_{m a x}$ is chosen to be 10 .

Further numerical computations for $\tan \beta=10$ determine that the limits on the boundary value of the stop mass from requiring positivity of the mass squared at the weak scale do not differ by more than a few percent from the case $\tan \beta=2.2$ (for the same values of the gluino and heavy scalar masses). However, the fine tuning of EWSB for the same gluino and stop mass is smaller for $\tan \beta=10$ as compared to $\tan \beta=2.2$. This is because, for $\tan \beta=10, \lambda_{t}$ is smaller than in the case $\tan \beta=2.2$. Hence the sensitivity of the weak scale value of $m_{H_{u}}^{2}$ to $m_{\tilde{t}, 0}^{2}$ and $M_{3,0}$ is smaller. Also, the $\tan ^{2} \beta /\left(\tan ^{2} \beta-1\right)$ factor in Eqn.(6.2) is smaller, further 
reducing the sensitivity of $m_{Z}^{2}$ to $m_{t, 0}^{2}$ and $M_{3,0}$. In other words, for $\tan \beta=10$, $m_{\bar{t}, \max }$ and $M_{3, \max }$ are larger so that $M_{S, \max }$ and, in turn, the limit on $\delta$ is larger. This can be seen in Figures 6.12 and 6.13.

\subsection{Summary}

This chapter has studied whether the SUSY flavor problem can be solved by making the scalars of the first and second generations heavy, with masses $M_{S}$ ( $\gtrsim$ few $\mathrm{TeV}$ ), without destabilizing the weak scale. If the scale, $M_{S U S Y}$, at which SUSY breaking is mediated to the SM scalars is close to the GUT scale, then the heavy scalars drive the light scalar (in particular the stop) mass squareds negative through two-loop RG evolution. In order to keep the mass squareds at the weak scale positive, the initial value of the stop (and other light scalar) soft masses, $m_{\tilde{f}_{i}, 0}$, must typically be $\gtrsim 1 \mathrm{TeV}$, leading to fine tuning in EWSB. Two new effects are included in this analysis: the effect of $\lambda_{t}$ in the RGEs which makes the stop mass squareds at the weak scale more negative and hence makes the constraint on the initial value stronger, and the QCD corrections to the SUSY box diagrams which contribute to $K-\bar{K}$ mixing.

Some results of the analysis for $M_{S U S Y}=M_{G U T}$ can be summarized as follows. The gluino mass (at the weak scale) is restricted to be less than about $710 \mathrm{GeV}$, so that the fine tuning of $m_{Z}^{2}$ with respect to the bare gluino mass, $M_{3,0}$, is not worse than $1 \%$. This requires that $M_{3,0} \lesssim 300 \mathrm{GeV}$. Also assume that $m_{H_{u}, 0}^{2}=0$ to maximize the value of the stop masses at the weak scale. Then for $\sqrt{\delta_{L L} \delta_{R R}}=0.22$, 

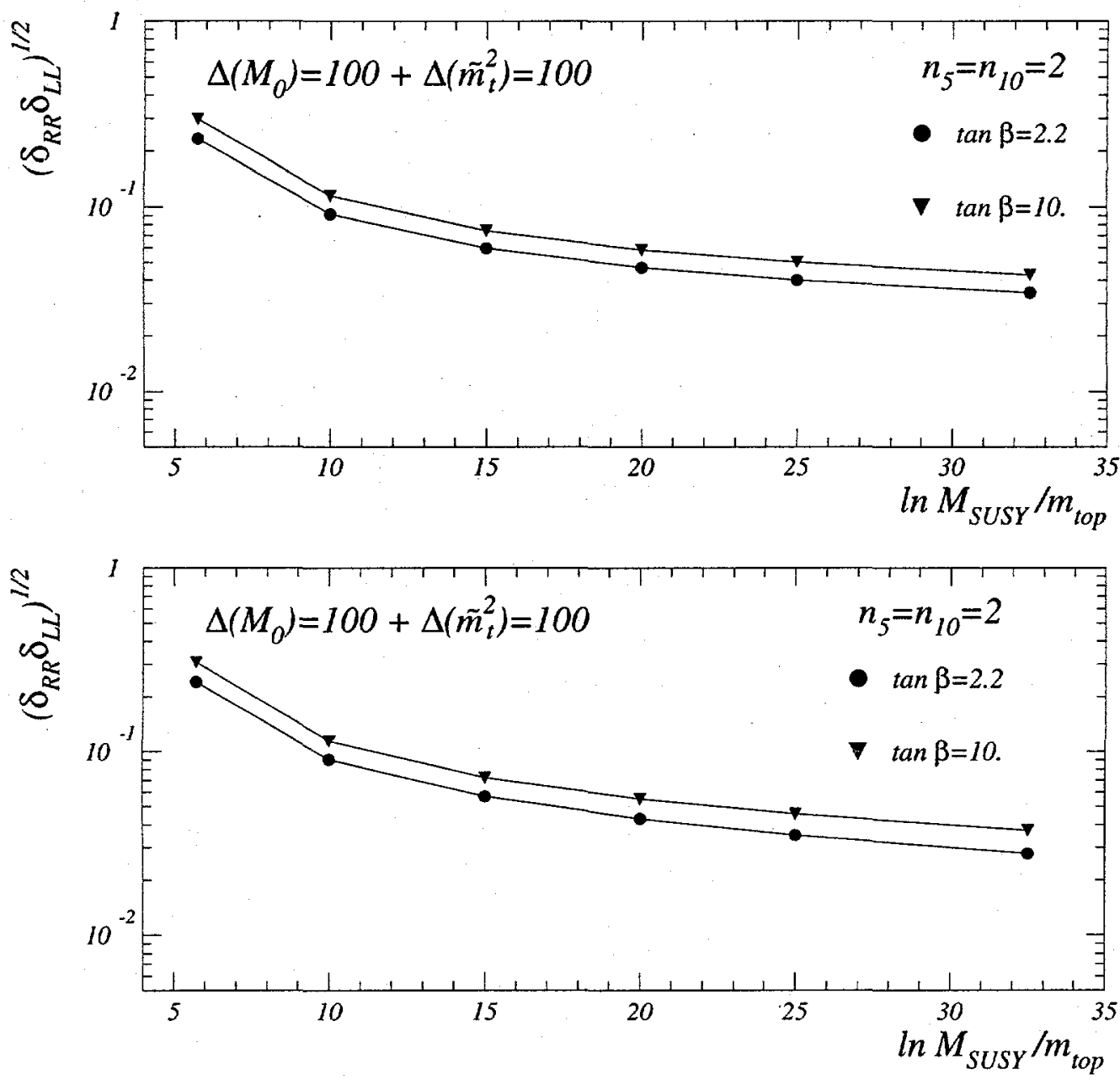

Figure 6.12: Maximum value for $\left(\delta_{L L} \delta_{R R}\right)^{1 / 2}$ that is consistent with $\Delta\left(m_{Z}^{2}, M_{3,0}\right)<$ 100, $\Delta\left(m_{Z}^{2}, m_{\tilde{t}, 0}^{2}\right)<100$ and $\left(\Delta m_{K}\right)_{S U S Y}<\left(\Delta m_{K}\right)_{e x p}$. Two boundary conditions are considered: $m_{H_{u}, 0}^{2}=0$ (top) and gauge-mediated relations (bottom). Two values for $\tan \beta$ are considered. The value of $A_{t, 0}$ was chosen to maximize the value of the stop masses at the weak scale. 

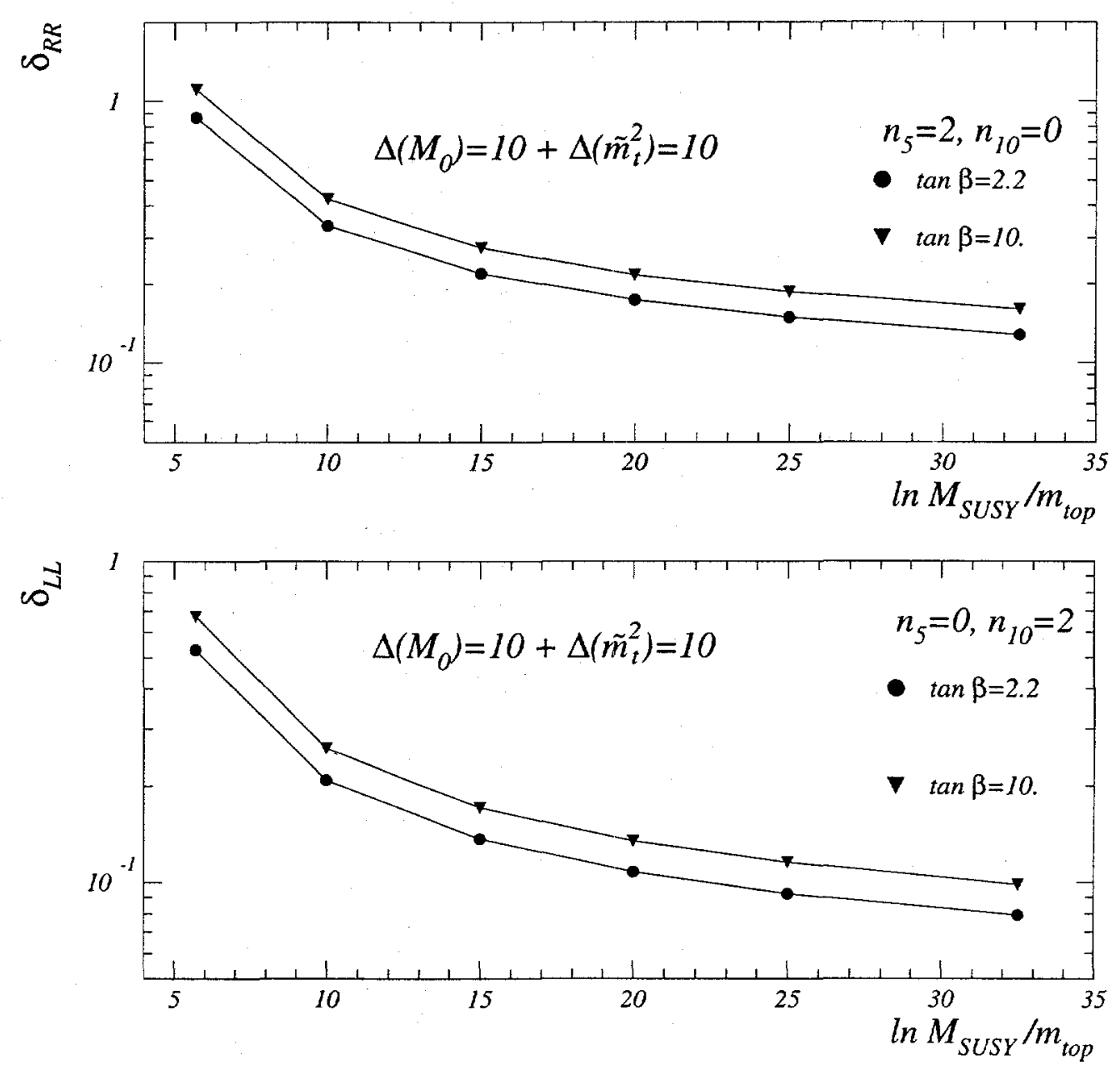

Figure 6.13: Maximum value for $\delta_{L L}, \delta_{R R}$ that is consistent with $\Delta\left(m_{Z}^{2}, M_{3,0}\right)<$ $10, \Delta\left(m_{Z}^{2}, m_{\tilde{t}, 0}^{2}\right)<10$ and $\left(\Delta m_{K}\right)_{S U S Y}<\left(\Delta m_{K}\right)_{\text {exp }}$. It was assumed that $m_{H_{u}, 0}^{2}=$ 0 . Two values for $\tan \beta$ are considered. The value of $A_{t, 0}$ was chosen to maximize the value of the stop masses at the weak scale. 
$M_{S} \geq 40 \mathrm{TeV}$ is required to be consistent with $\Delta m_{K}$. With these assumptions, this implies that for $M_{3,0}$ less than $1 \mathrm{TeV}, m_{\tilde{t}_{i}, 0}>6.5 \mathrm{TeV}$ is needed to not break color and charge at the weak scale. Even for $\sqrt{\delta_{L L} \delta_{R R}}=0.04$, it is found that $M_{S} \gtrsim 7$ $\mathrm{TeV}$ is needed. This implies that $m_{\tilde{t}, 0}>1 \mathrm{TeV}$ is required if $M_{3,0} \leq 300 \mathrm{GeV}$. This results in a fine tuning of $\sim 1 \%$. For $\delta_{L L}=1$ and $\delta_{K R}=0$, it is found that $M_{S} \gtrsim 30 \mathrm{TeV}$ and $m_{\tilde{t}, 0}>4.5 \mathrm{TeV}$. For $\delta_{L L}=0.22$ and $\delta_{R R}=0, M_{S} \gtrsim 7 \mathrm{TeV}$ and $m_{\tilde{t}, 0}>1 \mathrm{TeV}$ are found. For $\delta_{L L}=0$ and $\delta_{R R}=1, M_{S} \gtrsim 30 \mathrm{TeV}$ and $m_{\tilde{t}^{c}, 0}>2.5$ $\mathrm{TeV}$ are found. The constraints are weaker for smaller values of $\delta$. In a realistic model, $m_{H_{u}, 0}^{2}$ might be comparable to $m_{\tilde{t}, 0}^{2}$ and the constraints on $m_{\tilde{t}, 0}$ in this case are stronger. This is also discussed. It is noted that independent of the constraint from $K-\vec{K}$ mixing, this analysis can be used to check the phenomenological viability of any model that has heavy scalars. The phenomenological viability of the anomalous $D$-term solution is also discussed, and is found to be problematic.

The possibility that $M_{S U S Y}=M_{S}$ was also considered. In this case, there is no RG $\log$ enhancement of the negative contribution of the heavy scalar masses to the light scalar masses. For this case, the finite parts of the two-loop diagrams are computed and used as estimates of the two-loop contribution of the heavy scalars to the light scalar soft mass squareds. These results are combined with the constraints from $K-\bar{K}$ mixing to obtain lower limits on the boundary values of the stops. As an example, gauge-mediated SUSY breaking boundary conditions were assumed for the light scalars. If $n_{5} \neq n_{10}$ then one of the selectron masses, rather than the stop masses, provides the stronger constraint on $m_{\tilde{t}_{i}, 0}$ once gauge- 
mediated boundary conditions are used to relate $m_{\tilde{e}^{c}, 0}$ and $m_{\tilde{L}, 0}$ to $m_{\bar{t}_{i}, 0}$. Some of the results can be summarized as follows. The gluino mass at the weak scale is restricted to be less than about $2.3 \mathrm{TeV}$, again to avoid more than $1 \%$ fine tuning of $m_{Z}^{2}$ with respect to the gluino mass. For $\sqrt{\delta_{L L} \delta_{R R}}=.22$ it is found that $m_{\tilde{t}_{i}, 0} \geq$ $1.4 \mathrm{TeV}$ is required. The fine tuning of $m_{Z}^{2}$ with respect to the stop mass is $\sim 1.5 \%$ in this case. For the cases $\delta_{L L}=0$ and $\delta_{R R}=1$, and $\delta_{L L}=1$ and $\delta_{R R}=0$ it is found that $m_{\tilde{t}, 0} \gtrsim 1 \mathrm{TeV}$. As before, the constraints on $m_{\tilde{t}, 0}$ for smaller values of $\delta$ are weaker than $\sim 1 \mathrm{TeV}$. Again, the constraints in an actual model of this low-energy supersymmetry breaking scenario could be different, and the results discussed here should be treated as estimates only. The $C P$ violating constraints from $\epsilon$ are also discussed, and find that these limits increase by a factor of $\sim 12$ if $O(1)$ phases are present.

Finally, in section 6.5 the scale of supersymmetry breaking is varied between $50 \mathrm{TeV}$ and $2 \times 10^{16} \mathrm{GeV}$. Uppers bounds to $\delta$, that are consistent with positivity of the light scalar masses, naturalness in electroweak symmetry breaking, and $\left(\Delta m_{K}\right)_{\text {exp }}$, are obtained. These results are summarized in Figures 6.12 and 6.13. 


\section{Chapter 7}

\section{Dynamically Generating the Grand Unification}

\section{Scale}

Two models which generate the supersymmetric Grand Unification Scale from the strong dynamics of an additional gauge group are presented. The particle content is chosen such that this group confines with chiral symmetry breaking. Fields that are usually introduced to break the Grand Unified group appear instead as composite degrees of freedom and can acquire vacuum expectation values due to the confining dynamics. The models implement known solutions to the doublet-triplet splitting problem. The $S O(10)$ model only requires one higher dimensional representation, an adjoint. The dangerous colored Higgsino-mediated proton decay operator is naturally suppressed in this model to a phenomenologically interesting level. Neither model requires the presence of gauge singlets. Both models are only technically natural.

\subsection{Introduction}

One of the most beautiful ideas for physics beyond the Standard Model (SM) is the idea [13] that the gauge groups of the Standard Model (SM) unify into a single 
gauge group, the Grand Unified Theory (GUT). This would provide some common understanding for the diversity of particle content and parameters that constitute the Standard Model. That one generation of fermions can be accommodated by a single 16 of $S O(10)$ is too remarkable to be a coincidence! More indirect evidence for this framework is provided by the precision electroweak data. These suggest that the gauge couplings of the Standard Model unify at a high energy scale. In fact, a very good agreement with the data is obtained if softly-broken supersymmetry is realized close to the weak scale.

This naturally leads to a consideration of supersymmetric GUTs [65]. The scale of supersymmetric unification inferred from the data is $M_{G U T} \sim 2 \times 10^{16} \mathrm{GeV}$. Above this scale Nature may be described by a supersymmetric GUT. The value of this scale given by the data does not appear to be directly related to any other mass scale in Nature. The closest scale is the reduced Planck mass, $M=1 / \sqrt{8 \pi G_{N}}$, which is about a factor of 100 larger than the GUT scale. Most attempts at supersymmetric model building remain agnostic about the origin of the GUT scale, and simply put into the theory by hand both the scale and pattern of symmitry breaking. While this is technically natural in supersymmetric theories, it completely avoids the issues of the origin of the GUT symmetry breaking and the small value of $M_{G U T} / M$. This issue is particularly relevant if the scale $M$ is representative of a fundamental scale of new physics. If this is the case, then the small value of the supersymmetric Grand Unification scale compared to the Planck scale is perplexing. 
Some of these issues can be addressed by applying some of the recent developments in the strong dynamics of supersymmetric gauge theories [107]. In particular, the strong dynamics of an additional gauge group that confines with chiral symmetry breaking at a scale close to the GUT scale is considered. The idea of using strong dynamics to generate the supersymmetric GUT scale has only recently been explored $[108,109,110]$. This was first explored in Reference [108], where a dynamically generated superpotential with a runaway behavior is used to generate $M_{G U T} / M$. In Reference [110] the confining dynamics without chiral symmetry breaking is used in a novel manner to solve the doublet-triplet splitting problem. In that model though, a large top quark Yukawa coupling is only possible if the unification scale is uncomfortably close to the Planck scale. In Reference [109] the quantum confinement with chiral symmetry breaking is used to generate the GUT scale.

The idea of using strong supersymmetric dynamics to generate ratios of symmetry breaking scales has also been applied to flavor symmetries [111, 112]. The first phenomenological application of quantum confinement with chiral symmetry breaking in this context is given in Reference [112].

The outline of this chapter is as follows. Section 6.2 describes some features that are common to the models presented in section 6.3 and 6.4 . Section 6.3 introduces a model with an $S U(\hat{b})$ GUT group. Section 6.4 introduces the preferred model which has an $S O(10)$ GUT group. 


\subsection{Overview}

In the models presented in this chapter an extra gauge group $G_{C}$ is introduced and assumed to become strong at a scale $\Lambda \sim M_{G U T}$. The particle content of $G_{C}$ is chosen so that it confines with chiral symmetry breaking. This sector of the theory will be called the 'confining sector'. By identifying the GUT group, $G_{G U T}$, with a global symmetry of the confining sector, the composite fields of the confining sector are charged under the GUT group. For example, in the first model presented below, an adjoint of $S U(6)_{G U T}$ is composite. In the second model, a symmetric and antisymmetric tensor of $S O(10)_{G U T}$ is composite. This differs from the model of Reference [109], where the confining sector in that model does not contain particles charged under the GUT group. Below the scale of confinement, some of the composite fields will acquire vacuum expectation values (vevs) as a consequence of the dynamics of confinement. In the models presented here there is a discrete set of supersymmetric vacua. In one of these vacua the vevs of the composite fields break the GUT group; this together with some superpotential interactions lead to a phenomenologically acceptable vacuum. The small value of $M_{G U T} / M_{P L}$ is then understood as naturally arising from the dimensional transmutation of the small gauge coupling of $G_{C}$ at the Planck scale.

The simplest example of a supersymmetric gauge theory that exhibits confinement with chiral symmetry breaking is $S U(N)$ with $N$ flavors $Q+\bar{Q}$ and no superpotential [107]. This will be the model for the confining sector. It is con- 
jectured that below the scale of strong dynamics, $\Lambda$, of the $S U(N)$ group, the appropriate degrees of freedom are the confined "baryons" $B, \bar{B}$, and "mesons" $M$ of the $S U(N)$ group, where

$$
\begin{gathered}
M_{i}^{j} \sim \bar{Q}_{a}^{j} Q_{i}^{a} \sim(\bar{\square}, \square, 0) \\
B \sim \epsilon_{a_{1} \cdots a_{N}} Q_{i_{1}}^{a_{1}} \cdots Q_{i_{N}}^{a_{N}} \sim(\mathbf{1}, \mathbf{1}, 1) \\
\bar{B} \sim \epsilon^{a_{1} \cdots a_{N}} \bar{Q}_{a_{1}}^{i_{1}} \ldots \bar{Q}_{a_{N}}^{i_{N}} \sim(\mathbf{1}, \mathbf{1},-1) .
\end{gathered}
$$

The charges of the baryons and mesons under the global $S U(N) \times S U(N) \times U(1)_{B^{\prime}}$ are indicated in parentheses. The space of supersymmetric vacua for the baryons and mesons is described by [107]

$$
\operatorname{det} M-B \bar{B}=\Lambda^{2 N} .
$$

The left-hand side of this equation vanishes at the classical level as a consequence of the Bose statistics of the superfields $Q$ and $\bar{Q}$. Quantum corrections result in a non-vanishing value for the right-hand side. The important point is that along the supersymmetric vacua, some of the confined fields necessarily acquire vevs, breaking the global symmetry down to a subgroup. This conjecture satisfies two nontrivial consistency tests [107]: holomorphic decoupling of one flavor; and t'Hooft anomaly matching of the unbroken global symmetries.

In this chapter a diagonal subgroup of the global symmetry of the confining sector is gauged and identified with the GUT group. The mesons of the confining sector therefore transform under the GUT group. It will be assumed that the 
weakly gauging of a global symmetry of the confining sector does not affect the confining dynamics of $G_{C}$, and does not ruin the quantum modification with chiral symmetry breaking. This is a reasonable assumption since the GUT group is weakly gauged at the scale $\Lambda \sim M_{G U T} \sim 2 \times 10^{16} \mathrm{GeV}$.

Perhaps the most difficult problem in GUT model building is the origin of the doublet-triplet mass splitting. The excellent agreement between the measured and theoretically predicted value of $\sin ^{2} \theta_{W}$ assumes that the particle content below the unification scale contains the (supersymmetric) SM chiral matter content plus two electroweak Higgs doublets. In a minimal $S U(5)$ GUT, the Higgs fields fit into a 5 and $\overline{5}$ of $S U(5)$. The presence of the remaining particle content of these representations-the two colored Higgs triplets- much further than a few decades below the GUT scale completely ruins this agreement. More generally, requiring that there exists one large split $S U(5)$ representation is a strong constraint on model building. The models presented in this chapter implement two known solutions to this problem: the Higgs as "pseudo-Goldstone bosons" [113] and the "Dimopoulos-Wilzcek" [114] missing vevs mechanism. The latter solution is implemented in an $S O(10)$ GUT gauge group, whereas the former is based upon an $S U(6)$ GUT group.

In the models presented here the quantum confinement is therefore not directly responsible for the doublet-triplet splitting. The structure outlined above must be supplemented with a non-vanishing superpotential in order to implement the doublet-triplet splitting. A non-vanishing superpotential must be added in any 
case: a generic point on the quantum modified constraint breaks $S U(N) \times U(1)_{B^{\prime}}$ down to $U(1)^{N-1}$. This provides too much symmetry breaking. A point that only breaks to a larger subgroup is therefore an enhanced symmetry point, corresponding to a particular choice of the vevs of $M$ and $B$. At the enhanced symmetry point, there are many massless particles in addition to the Nambu-Goldstone multiplets. These correspond to the would-be Goldstone bosons of the more generic symmetry breaking pattern, and at the enhanced symmetry point, transform as adjoints under the unbroken gauge group. These particles must acquire masses from additional superpotential interactions. These superpotential interactions then explicitly break the global symmetry of the confining sector down to $G_{G U T} \times U(1)_{B^{\prime}}$.

It is then a concern whether the presence of this superpotential might destabilize the confinement and chiral symmetry breaking. The form of the superpotential for the fundamental fields of the group $G_{C}, Q, \bar{Q}$, and any fields $\psi_{M}$ not charged under $G_{C}$, in the two models presented here is

$$
W=W_{C}\left(Q, \bar{Q}, \psi_{M}\right)+W_{M}\left(\psi_{M}\right)
$$

The superpotential $W_{C}$ involving the confining fields will by fiat contain only non-renormalizable operators, suppressed by a scale assumed to be either the Planck mass or reduced Planck mass. If confinement occurs, the coefficient $c$ of an operator with mass dimension $d$ in the low-energy theory that arose from an operator with $N(\bar{Q} Q)$ s in the high energy theory is expected to be

$$
c \sim \lambda \times \Lambda^{N} / M^{N-d},
$$


where $\lambda$ is a constant that is expected to be of order unity. For the models considered below, $d=-1,0$ or $1, N$ is 1 or 2 , and $N-d$ is positive. Since these coefficients are suppressed by powers of $\Lambda / M$, the presence of these terms in the superpotential is a small perturbation to the quantum confinement. It is then reasonable to expect that these operators do not destroy the quantum confinement with chiral symmetry breaking. This assumption will be made for the remainder of the chapter.

In the usual GUT model building framework, the unification of the gauge couplings can be significantly affected by the presence of $M^{-1}$ suppressed operators [115]. In an $S U(5)$ model, for example, the gauge field-strength tensor $F$ can have non-renormalizable interactions with an adjoint $\Sigma$. The operator $c \Sigma F F / 4 M$ results in a tree-level relative shift of the gauge couplings $1 / g_{i}^{2}$ that is approximately $c M_{G U T} / M$. This translates into a shift in the low-energy value of $\sin \theta_{W}^{2}$ that for $M / M_{G U T}=20$ is $\Delta \sin \theta_{W}^{2}\left(M_{Z}\right) \sim \pm$ few $\times c \times 10^{-3}$. In the GUT models presented in this chapter, some of the higher dimensional representations are composite. For the composite fields, the gravitational smearing operator arises from a higher dimension operator in the fundamental theory. The coefficient of this operator below the confinement scale then contains an additional suppression of $\Lambda / M$. This extra factor completely suppresses the smearing effect unless the coefficient of the operator in the fundamental theory is unnaturally large-of $O\left(M / M_{G U T}\right)$-and $M_{G U T} / M$ is $\sim 1 / 20$. Non-composite higher dimensional fields can contribute to the gravitational smearing. In the $S O(10)$ model, it turns out 
that these contributions are completely negligible.

This section concludes with a discussion of some technical issues that occur throughout the chapter. Implicit in the discussion that follows will be the assumptions that (global) supersymmetry is unbroken, and that the non-trivial Kähler potential has a strictly positive definite Kähler metric [112].

Supersymmetric minima are found by looking for solutions to the $F$-flatness equations $0=F_{i}=\partial_{\phi_{i}} W$ for the confined and $\psi_{M}$ fields. This is rather naive, since the vevs of the fields will typically be $O(\Lambda)$ and the Kähler potential is non-calculable for these field values. It is not clear then that the "baryons" and "mesons" are the correct degrees of freedom. For the purposes of determining the existence of supersymmetric vacua with a particular pattern of symmetry breaking, however, the last assumption of the previous paragraph is sufficient [112]. With these assumptions, a supersymmetric vacuum found using a trivial Kähler potential will remain supersymmetric for the non-trivial Kähler potential. The spectrum of the particle masses is also important for phenomenology. For this, knowledge of the Kähler potential is required. Despite the absence of this information, a few important points about the mass spectrum can be extracted from the superpotential [112]. For example, a particle that is massless (zero eigenvector of $F_{i, k}$ ) in the case of a canonical Kähler potential for the confined fields will remain massless in the case of a non-trivial Kähler potential. Similarly, a massive particle in the trivial Kähler potential will remain massive for a non-trivial Kähler potential. So the mass spectrum computed by assuming a trivial Kähler potential 
will be used to check that the superpotential with a non-trivial Kähler potential results in superheavy masses to all the particles that should have superheavy masses.

In the models presented here, the superpotential interactions that involve the confining fields occur from higher dimension operators, so that after confinement the superpotential coupling of those operators is $\tilde{\lambda} \sim \lambda(\Lambda / M)^{n} \ll \lambda$, with $\lambda \sim$ $O(1)$. Particles that acquire their mass from these operators will then have masses somewhat below the GUT scale. These masses remain uncalculable though, since they should be computed at a scale that is comparable to the vev that is generating the mass, which in this case is $O(\Lambda)$.

The one-loop prediction for $\sin ^{2} \theta_{W}$ is modified by the presence of these light states below the GUT scale since they do not in general form complete $S U(5)$ representations. An attempt at quantifying this correction is made by assuming that the naive calculation-i.e. assuming a canonical Kähler potential-of the spectrum gives the correct mass spectrum to within a factor of a few (times unity), and further, that the correction to $\sin ^{2} \theta_{W}$ from particles with masses much smaller than tle confinement scale is well-approximated by the usual one-loop computation. The corrections from particles with masses near the confinement scale are not calculable and not discussed.

Finally, in the two models presented here certain operators allowed by the gauge symmetries of the theory must be absent from the superpotential in order not to ruin the doublet-triplet splitting mechanisms. All the dangerous operators 
cannot be forbidden by any global symmetries, since some of them will have the same quantum numbers as other operators that are required to be present in the superpotential. If these models were only the effective theory of some more fundamental field theory, then the dangerous operators could perhaps be generated at the tree-level by integrating out some heavy particles at the scale $M$. In this case however, the full theory above the Planck scale is not known and probably not a field theory. It is then possible that the full theory could be responsible for the absence of these dangerous operators, even though from the low-energy theory they cannot be forbidden by any symmetries.

\section{$7.3 \quad S U(6) \times S U(6)$}

The gauge group is $S U(6)_{C} \times S U(6)_{G U T}$ where one factor of $S U(6)$ is the confining group $G_{C}$, and the other factor is the SM unified gauge group. Consider six flavors, $Q+\bar{Q}$ of $S U(6)$ that are also charged under the $S U(6)_{G U T}$. Further, introduce two Higgs fields $H, \bar{H}$, and an adjoint $\Sigma_{N}$ that are charged under only the $S U(6)_{G U T}$. The particle content under $S U(6)_{C} \times S U(6)_{G U T}$ is then

$$
\begin{aligned}
Q & \sim(\mathbf{6}, \overline{\mathbf{6}}), \\
\bar{Q} & \sim(\overline{\mathbf{6}}, \mathbf{6}), \\
H & \sim(\mathbf{1}, \mathbf{6}), \\
\bar{H} & \sim(\mathbf{1}, \overline{\mathbf{6}}), \\
\Sigma_{N} & \sim(\mathbf{1}, \mathbf{3 5}) .
\end{aligned}
$$


The $S U(6)_{C}$ group is assumed to confine at a scale $\Lambda \sim M_{G U T}$ with a quantum modified constraint. In this case the confined "meson" $M_{i}^{j} \sim \bar{Q}_{i}^{a} Q_{a}^{j} \sim \mathbf{3 5}+\mathbf{1}$ under the $S U(6)$ GUT symmetry. The "baryons" $B \sim \epsilon Q^{6}$ and $\bar{B} \sim \epsilon \bar{Q}^{6}$ are singlets under the $S U(6)_{G U T}$ group. No gauge singlets are required in the fundamental theory.

The superpotential in terms of the fundamental fields is chosen to be

$$
W_{0}=\frac{1}{2} \lambda_{1} \operatorname{tr}(Q \bar{Q})^{2} / M+\lambda_{3} H \bar{H} \operatorname{tr}(Q \bar{Q}) / M+\lambda \operatorname{tr}\left(\Sigma_{N}^{2} Q \bar{Q}\right) / M+\bar{g}(H \bar{H}) \operatorname{tr} \Sigma_{N}^{2} / M
$$

The scale $M$ is assumed to be the reduced Planck mass $\sim 2 \times 10^{18} \mathrm{GeV}$. The trace sums over the $S U(6)_{G U T}$ indices. All the dimensionless parameters are assumed to be of order unity. This superpotential contains the minimum number of interactions necessary (as shown below) to successfully implement in the phenomenologically preferred vacuum the doublet-triplet splitting and give GUT scale masses to all the other particles. A more general superpotential is allowed provided that: (1) Only non-renormalizable operators involving $Q, \bar{Q}$ are allowed. (2) To keep the Higgs doublets light, the superpotential that only involves the $35 \mathrm{~s}$ and the $H$, $\bar{H}$ fields must preserve a $S U(6) \times S U(6)$ global symmetry [113]. The operators $\bar{H}(\bar{Q} Q)^{n} H$ and $\bar{H}\left(\Sigma_{N}\right)^{n} H$, for example, must be absent. (3) Supersymmetry is not spontaneously broken.

After confinement occurs, the superpotential written in terms of the confined 
fields $\Sigma \sim 35$ and $\sigma \sim 1$, i.e. $\bar{Q}_{i}^{a} Q_{a}^{j} \sim \Lambda \Sigma_{i}^{j}+\Lambda \sigma \delta_{i}^{j} / \sqrt{6}$, is

$$
\begin{aligned}
W_{0}= & \mathcal{A}\left(\operatorname{det}(\Sigma+\sigma / \sqrt{6})-B \bar{B}-\Lambda^{6}\right)+\frac{1}{2} \tilde{\lambda}_{1} \Lambda \operatorname{tr} \Sigma^{2}+\frac{1}{2} \tilde{\lambda}_{2} \Lambda \sigma^{2} \\
& +\tilde{\lambda}_{4} \operatorname{tr} \Sigma_{N}^{2} \Sigma+\tilde{\lambda}_{5} \sigma \operatorname{tr} \Sigma_{N}^{2}+(H \bar{H})\left(\tilde{\lambda}_{3} \sigma+\bar{g} \operatorname{tr} \Sigma_{N}^{2} / M\right)
\end{aligned}
$$

It is expected that

$$
\tilde{\lambda}_{1,2} \sim \lambda_{1} \Lambda / M, \quad \tilde{\lambda}_{3} \sim \lambda_{3} \Lambda / M, \tilde{\lambda}_{4,5} \sim \lambda \Lambda / M
$$

as an estimate of the size of the couplings in the confined description. The quantum modified constraint has been added using a Lagrange multiplier $\mathcal{A}$. This superpotential contains all the non-perturbative (superpotential) information from the strong $S U(6)_{C}$ dynamics. It is interesting that in this case a term in the superpotential for $Q \bar{Q}$ that generates a cubic term $\operatorname{tr} \Sigma^{3}$ is not required. In most supersymmetric GUT models, the cubic term is required to obtain a non-trivial vacuum. In this case, it is the interaction $\mathcal{A} \operatorname{det}(\Sigma+\sigma)$ from the quantum modified constraint that balances the mass terms to obtain a non-trivial supersymmetric vacuum.

The $F$-flatness equations are

$$
\begin{aligned}
& \operatorname{det}(\Sigma+\sigma / \sqrt{6})-B \bar{B}=\Lambda^{6}, \\
& 0=F_{\bar{B}}=\mathcal{A} B, 0=F_{B}=\mathcal{A} \bar{B}, \\
& 0=F_{\bar{H}}=\left(\tilde{\lambda}_{3} \sigma+g \operatorname{tr} \Sigma_{N}^{2} / M\right) H, \\
& 0=F_{H}=\left(\tilde{\lambda}_{3} \sigma+g \operatorname{tr} \Sigma_{N}^{2} / M\right) \bar{H},
\end{aligned}
$$




$$
\begin{aligned}
0=F_{\sigma}=\tilde{\lambda}_{3} H \bar{H}+ & \tilde{\lambda}_{2} \Lambda \sigma+\frac{\mathcal{A}}{\sqrt{6}} \operatorname{det}(\Sigma+\sigma / \sqrt{6}) \operatorname{tr}(\Sigma+\sigma / \sqrt{6})^{-1}+\tilde{\lambda}_{5} \operatorname{tr} \Sigma_{N}^{2}, \\
0=F_{\Sigma}= & \tilde{\lambda}_{1} \Lambda \Sigma+\tilde{\lambda}_{4}\left(\Sigma_{N}^{2}-\frac{1}{6} \operatorname{tr} \Sigma_{N}^{2}\right) \\
& +\mathcal{A} \operatorname{det}(\Sigma+\sigma / \sqrt{6})\left((\Sigma+\sigma / \sqrt{6})^{-1}-\frac{1}{6} \operatorname{tr}(\Sigma+\sigma / \sqrt{6})^{-1}\right), \\
0=F_{\Sigma_{N}}= & \tilde{\lambda}_{4}\left(\Sigma_{N} \Sigma-\frac{1}{6} \operatorname{tr} \Sigma_{N} \Sigma\right)+\tilde{\lambda}_{5} \sigma \Sigma_{N}+g(H \bar{H}) \Sigma_{N} / M .
\end{aligned}
$$

In addition to the phenomenologically preferred vacuum, these equations include other discrete solutions. In some of these solutions $S U(6)_{G U T}$ is unbroken. For example, a solution with $\sigma$ and $\mathcal{A}$ non-zero, and all other vevs equal to zero, exists. So although the preferred vacuum is discrete, it is assumed that it was selected in the early history of the universe. This could occur if, for example, the preferred vacuum is a global minimum of the scalar potential after supersymmetry breaking effects are included.

To break $S U(6)$ down to the SM gauge group, it is assumed that ${ }^{1}$

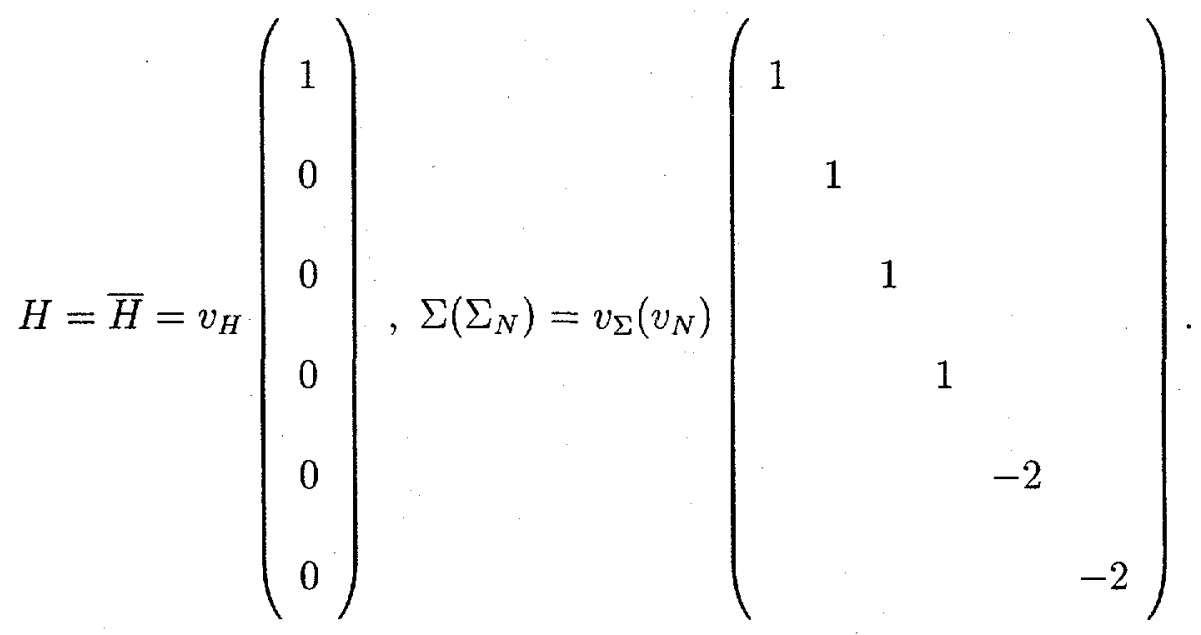

\footnotetext{
${ }^{1} H=\bar{H}$ is required by $S U(6)_{G U T} D$-flatness.
} 
The vevs $\mathcal{A}, \sigma, v_{\Sigma}, v_{N}$ and $v_{H}$ are the solutions to

$$
\begin{gathered}
0=\left(\tilde{\lambda}_{3} \sigma+12 g v_{N}^{2} / M\right) v_{H}, \\
0=\left(\tilde{\lambda}_{5} \sigma+g v_{H}^{2} / M-\tilde{\lambda}_{4} v_{\Sigma}\right) v_{N}, \\
0=\frac{1}{3} \mathcal{A} K(a-b)+\tilde{\lambda}_{1} \Lambda v_{\Sigma}-\tilde{\lambda}_{4} v_{N}^{2}, \\
0=2 \frac{\mathcal{A} K}{\sqrt{6}}(2 a+b)+\tilde{\lambda}_{2} \Lambda \sigma+\tilde{\lambda}_{3} v_{H}^{2}+12 \tilde{\lambda}_{5} v_{N}^{2},
\end{gathered}
$$

and for $\mathcal{A} \neq 0, \operatorname{det}(\Sigma+\sigma / \sqrt{6})=\Lambda^{6}$. The quantities $a, b$ and $K$ are defined to be $a^{-1} \equiv v_{\Sigma}+\sigma / \sqrt{6}, b^{-1} \equiv-2 v_{\Sigma}+\sigma / \sqrt{6}$ and $K \equiv \operatorname{det}(\Sigma+\sigma / \sqrt{6})=a^{-4} b^{-2}$. In Appendix $\mathrm{C}$ it is demonstrated that a discrete solution exists with $\mathcal{A} \sim(\Lambda / M) \Lambda^{-3}$ and with all vevs non-zero and of $O(\Lambda)$. Thus at this vacuum the vevs of the baryon fields are forced to the origin.

This vacuum implements the Higgs as "pseudo-Goldstone bosons" solution to the doublet-triplet splitting problem [113]. This mechanism is now briefly described. Firstly, the scalar potential for $H, \bar{H}$ and $\Sigma, \Sigma_{N}$ has a $U(6) \times S U(6)$ global symmetry. The $U(6)$ acts on $H$ and $\bar{H}$, whereas the $S U(6)$ symmetry acts on $\Sigma$ and $\Sigma_{N}$. For the vacuum in Eqn.(7.16), the global $U(6) \times S U(6)$ symmetry is broken to $[S U(5)] \times[S U(4) \times S U(2) \times U(1)]$ by the vevs of $H, \Sigma$ and $\Sigma_{N}$. The unbroken gauge group is then $S U(3)_{C} \times S U(2) \times U(1)_{Y}$. The breaking of the gauge symmetry results in $23 \mathrm{Nambu-Goldstone} \mathrm{boson} \mathrm{multiplets;} \mathrm{the} \mathrm{breaking} \mathrm{of}$ the $S U(6) \times U(6)$ results in 27 Goldstone boson multiplets. So all but 4 of the Goldstone bosons acquire mass of $O\left(M_{G U T}\right)$ from the super-Higgs mechanism. 
To see that these four pseudo-Goldstone bosons carry the quantum number charges of two electroweak doublets, first note that under $S U(4) \times S U(2), \mathbf{3 5}=$ $(4,2)+(\overline{4}, 2)+(15,1)+(1,3)+(1,1)$. Inspecting the vevs of $\Sigma$ and $\Sigma_{N}$, the combination $\tilde{v}_{\Sigma} \tilde{\Sigma} \equiv v_{\Sigma} \Sigma+v_{N} \Sigma_{N}$ of the fields $(4,2)$, and of the fields $(\overline{4}, 2)$, in $\Sigma$ and $\Sigma_{N}$ are the Goldstone bosons of the breaking of one global $S U(6)$ symmetry. Since $S U(3)_{C}$ is embedded in $S U(4)$, these Goldstone bosons contain two electroweak doublets. The Goldstone bosons of the $S U(6) \rightarrow S U(5)$ breaking are $\mathbf{5}+\overline{\mathbf{5}}+\mathbf{1}$ of $S U(5)$, and also contain two electroweak doublets. The combination $3 \tilde{v}_{\Sigma} \tilde{\Sigma}+v_{H} H$ of electroweak Higgs doublets are the fields eaten by the super-Higgs mechanism. The orthogonal combination remain massless and are the two Higgs doublets of the SM. The non-renormalization theorems of supersymmetry guarantee that these fields remain massless to all orders in perturbation theory.

The fields in the adjoint $(\mathbf{1 5}, \mathbf{1})$ and $(\mathbf{1}, \mathbf{3})$ of both $\Sigma$ and $\Sigma_{N}$, as well as the remaining combination of $(4,2)$, and of $(\overline{4}, 2)$, in $\Sigma$ and $\Sigma_{N}$ orthogonal to $\tilde{\Sigma}$, do not correspond to any broken generators and must acquire their masses from the superpotential interactions. It is conveinent to express the $S U(5)$ or SM charge assignments of this particle content: one complete $\mathbf{2 4}$ and $\mathbf{5}+\overline{\mathbf{5}}$ of $S U(5) ; 4$ singlets; and one $(8,1,0)+(1,3,0)+(3,1,-1 / 3)+(\overline{3}, 1,1 / 3)$. A naive estimate for the masses of the physical fields is obtained by computing the fermion mass matrix assuming a canonical Kähler potential. The results are presented in Appendix C, and are summarized here. All the fields have a mass $m \sim \Lambda^{2} / M$, a consequence of the suppression of the superpotential couplings for the confined theory. 
These light fields affect the unification of the gauge couplings and may in principle also mediate proton decay. The corrections to $\sin ^{2} \theta_{W}$ are discussed first. These corrections occur from two sources. There could be large threshold corrections from the strong dynamics occuring at $\Lambda$. These are non-calculable and will not be considered. The other is from the light states $(8,1,0),(3,1,0)$, $(\overline{3}, 1,1 / 3)$ and $(\mathbf{3}, \mathbf{1},-1 / 3)$ which have a mass $m \sim \Lambda^{2} / M$. The correction to $\sin ^{2} \theta_{W}$ from these light states, using a naive one-loop running approximation from $M_{G U T}$ to their masses is

$$
\Delta \sin ^{2} \theta_{W}=-\frac{\alpha_{e m}}{5 \pi} \ln M_{G U T} / m \sim-0.003 \times \frac{\ln \left(M_{G U T} / m\right)}{\ln 200} .
$$

The reason ${ }^{2}$ for the small correction is that the shift in $\sin ^{2} \theta_{W}$ is dominated by the light $(\overline{3}, 1,1 / 3)$ and $(3,1,-1 / 3)$ states. This is because the shift from the $(8,1)$ and $(1,3)$ states almost cancel. Recall that a sufficient condition for the prediction for $\sin ^{2} \theta_{W}$ to be unchanged by the presence of some extra matter at a scale $m$ is that $\left(\delta b_{3}-\delta b_{2}\right) /\left(\delta b_{2}-\delta b_{1}\right)=\left(b_{3}-b_{2}\right) /\left(b_{2}-b_{1}\right)$, independent of $m$. For an adjoint of $S U(3)$ and $S U(2), \delta b_{3}=3, \delta b_{2}=2$ and $\delta b_{1}=0$. In this case the LHS of this condition is 1 and the RHS is $\frac{5}{7} \times 2$, which is close to 1 . The other light states form approximate complete $S U(5)$ representations and do not significantly affect the gauge-coupling unification. The theoretical prediction without the light fields, $\sin ^{2} \theta_{W} \sim .233 \pm O\left(10^{-3}\right)$ [116], is a little larger than the measured value of $0.231[36]$. The effect of these light states is to shift the prediction in the correct direction. The uncertainty in the uncalculable corrections $\operatorname{tos}^{2} \sin _{W}$, however, are

\footnotetext{
${ }^{2}$ The author thanks N. Arkani-Hamed for this observation.
} 
probably of the same order, with an unknown sign.

The problem of forbidding operators of the form $\mathcal{O}_{n} \sim \bar{H}(Q \bar{Q})^{n} H$ is discussed next. These operators explicitly break the $U(6) \times S U(6)$ symmetry of the scalar potential. Consequently, if these operators are present they could give too large of a mass to the electroweak Higgs doublets. In this model, the term $\bar{H} H \operatorname{tr} \bar{Q} Q$ occurs in the superpotential. Any symmetry that allows this term also allows the term $\bar{H}(\bar{Q} Q) H$ in the superpotential. This operator ruins the doublet-triplet splitting, so it must be assumed that this term is absent. Higher dimensional operators must also be forbidden. Since the confinement introduces additional suppressions of $O\left(\Lambda^{n} / M^{n}\right)$, only a few of the first higher dimensional operators must be absent. More concretely, requiring that $\mathcal{O}_{n}$ not result in a mass for the Higgs superfields that is larger than a $\mathrm{TeV}$ and assuming that $\Lambda / M_{P L} \sim 1 / 200$, implies that only the first three ( $n=1,2$ and 3 ) higher dimensional operators must be forbidden. Operators of the type $\bar{H}\left(\Sigma_{N}\right)^{n} H$ are also dangerous and must be absent.

At this point it is probably not clear what role the extra adjoint plays in this model. In fact, this field is not needed to obtain an acceptable spectrum for the massive fields. It is introduced instead to obtain a large top quark Yukawa coupling. In order for the top quark not to have an irrelevant Yukawa coupling, it is necessary that the Yukawa interactions between the top quark and the Higgs doublet explicitly break the global $S U(6) \times U(6)$ symmetry. The top quark must therefore couple to both $H$ and $\Sigma$. If $\Sigma$ is composite, then such a coupling cannot be of order unity; rather, it will be suppressed by $\Lambda / M$. The top quark must 
therefore interact with a fundamental $\Sigma$.

The large top quark Yukawa coupling arises from considering the following embedding of the SM chiral fields [117] . The chiral matter content is one $\mathbf{2 0}$, $3 \times \mathbf{1 5}$ and $6 \times \overline{\mathbf{6}}$. The $S U(5)$ decomposition of these fields is, $\mathbf{2 0}=\mathbf{1 0}+\overline{\mathbf{1 0}}$, $15=10+5$ and $\overline{6}=\overline{5}+1$. The three $\overline{5}$ s of the SM are contained in three of the $\overline{\mathbf{6}} \mathrm{s}$, and the other 3 , call them $\overline{\mathbf{6}}^{\prime}$, acquire mass at the GUT scale. The first two generation $10 \mathrm{~s}$ are contained in two of the $15 \mathrm{~s}$, and the third generation 10 is a linear combination of the 10 in the 20 and the 10 in the remaining $15 \equiv \mathbf{1 5}_{3}$. This spectrum is obtained from the superpotential [117]

$$
W_{t o p}=\lambda 20 \Sigma_{N} 20+\lambda^{\prime} 20 H 15_{3}+\lambda_{i j}^{\prime \prime} \bar{H} 15_{i} \overline{\mathbf{6}}_{j}^{\prime} .
$$

The vev of $\bar{H}$ gives GUT-sized Dirac masses to the 5 and $\overline{5}$ fields in the $315 \mathrm{~s}$ and $3 \overline{\mathbf{6}}^{\prime} \mathrm{s}$. From the vevs of $\Sigma_{N}$ and $H$, a linear combination of the 10 in the 20 and the 10 in $\mathbf{1 5}_{3}$ acquires a GUT-sized Dirac mass with the $\overline{\mathbf{1 0}}$ in the $\mathbf{2 0 .}$ The orthogonal combination is the third generation 10 and remains massless. In sum, this superpotential leaves $3(\mathbf{1 0}+\overline{\mathbf{5}})$ s massless. The large top quark Yukawa coupling arises from the first two interactions.

The $(3,1,1 / 3)$ and $(\overline{3}, 1,-1 / 3)$ fields have a Dirac mass somewhat below the GUT scale. Whether they may mediate proton decay at too large of a rate is then a concern. Since the top quark couples to these fields through the $20 \Sigma_{N} 20$ interaction, it naively appears that a dangerous proton decay operator is generated by integrating out these heavy fields, and then rotating the top quark to the mass 
basis. For this operator to be generated, however, a coupling of $\Sigma_{N}$ or $\Sigma$ to a $\overline{5}$ of $S U(5)(\overline{6}$ of $S U(6))$ is required. Such a coupling is not present in the superpotential of Eqn.(7.22). So this issue depends crucially on the origin of the other fermion masses. For example, if all the fermion masses arise from interactions with $H$ and $\bar{H}$, then a dangerous proton decay operator is not generated by the exchange of these states [117].

An upper bound on $M$ is determined by the value of the Landau pole of the $S U(6)_{G U T}$ gauge coupling. The $S U(6)$ coupling at the scale $M$ is then

$$
\alpha_{S U(6)_{G U T}}^{-1}(M)=24-\frac{43}{11 \pi} \ln \Lambda / m_{I}-\frac{7}{2 \pi} \ln M / \Lambda .
$$

The first logarithm is the contribution to the GUT gauge coupling at the GUT scale from the particle content with mass $m$; the second logarithm is the contribution of the full $S U(6)$ particle content to the running of the gauge coupling above $\Lambda$. Inserting $m \sim \Lambda^{2} / M$ and requiring that $\alpha_{G U T}^{-1}(M) \geq 1$ implies $\ln M / \Lambda \leq 10$.

$7.4 S U(10) \times S O(10)$

The gauge group is $S U(10)_{C} \times S O(10)$. The $S U(10)_{C}$ group is the confining gauge group, and the Grand Unified group is $S O(10)$. The particle content is

$$
\begin{aligned}
Q & \sim(10,10) \\
\bar{Q} & \sim(\overline{10}, 10), \\
A & \sim(1,45), \\
16 & \sim(1,16),
\end{aligned}
$$




$$
\begin{aligned}
& \overline{\mathbf{1 6}} \sim(1, \overline{\mathbf{1 6}}), \\
& T_{1} \sim(1,10), \\
& T_{2} \sim(1,10) .
\end{aligned}
$$

This particle content is rather economical as it requires only one higher dimensional representation, an adjoint, and no gauge singlets ${ }^{3}$. It is assumed that the $S U(10)_{C}$ group confines at a scale $\Lambda \sim M_{G U T}$ with a quantum modified constraint. In this case the confined "meson" $M_{i}^{j} \sim \bar{Q}_{i}^{a} Q_{a}^{j} \sim \mathbf{4 5}+\mathbf{5 4}+\mathbf{1}$ under the $S O(10)$ GUT symmetry. The fields are labeled $S \sim 54, A^{\prime \prime} \sim 45$ and $\sigma \sim 1$. The "baryons" $B \sim \epsilon Q^{6}$ and $\bar{B} \sim \epsilon \bar{Q}^{6}$ are singlets under the $S O(10)_{G U T}$ group.

The superpotential in the fundamental theory is chosen to be

$$
\begin{aligned}
W= & \lambda_{1} T_{1} A T_{2}+\lambda_{2} T_{2}(\bar{Q} Q) T_{2} / M+\lambda_{3} \overline{\mathbf{1 6}}(\bar{Q} Q) \Sigma 16 / \bar{M}+\lambda_{4} \overline{\mathbf{1 6}} 16 \operatorname{tr}(\bar{Q} Q) / M \\
& +\lambda_{5} \operatorname{tr}(\bar{Q} Q)^{2} / M+\lambda_{7} A^{2}(\bar{Q} Q) / M+\lambda_{11} \overline{\mathbf{1 6}}(Q \bar{Q})_{A S} A \Sigma 16 / M^{2},
\end{aligned}
$$

where $\Sigma_{i j}=\left[\Gamma_{j}, \Gamma_{i}\right] / 4 i$ are the generators of $S O(10)$ in the spinorial representation. The subscript "AS" indicates that only the anti-symmetric contribution of $Q \bar{Q}$ is allowed to be present; the symmetric contribution spoils the doublet-triplet splitting. It is technically natural for only the anti-symmetric contribution to be present; the full theory above the Planck scale must be responsible for the absence of the symmetric operator. The operators $T_{1}(\bar{Q} Q)^{n} T_{1}$ must also be absent.

The renormalizable and $M^{-1}$ suppressed operators appearing in $W$ are all re-

\footnotetext{
${ }^{3}$ Also see Reference [118] for an economical model. In this model though, the origin of the unification scale is not addressed.
} 
quired: (i) The operators $\propto \lambda_{1}, \lambda_{2}$ are required for the doublet-triplet splitting. (ii) The operator $\propto \lambda_{7}$ arranges the vev of $A$ to be in the "Dimopoulos-Wilzcek" (DW) form [114], required to perform the doublet-triplet splitting. (iii) The operators $\propto \lambda_{3}$ and $\lambda_{4}$ are necessary to break the rank of the group. (iv) The operator $\propto \lambda_{5}$ is necessary to fix all the vevs. This point is made clear later. (v) The operator $\propto \lambda_{11}$ is required to give mass to some fields charged under the SM. This point is also discussed later. Although this operator is linear in $A$, the DW for $A$ is not ruined because this operator does not contribute to the $F$ flatness equations 4. The choice for this operator is not unique; other operators that are linear in $A^{2}$ are possible, but they are higher dimensional. It is non-trivial that with this choice for $W$, the low-energy particle content only contains the SM fields and their superpartners.

After confinement occurs the superpotential is

$$
W=W_{H}+W_{D W}+W_{m i x}
$$

with

$$
\begin{aligned}
W_{H} & =\lambda_{1} T_{1} A T_{2}+\tilde{\lambda}_{2} T_{2} S T_{2}+\tilde{\lambda}_{3} \sigma T_{2} T_{2} \\
W_{D W} & =\frac{1}{2} \tilde{\lambda}_{9} A^{2} S+\frac{1}{2} \tilde{\lambda}_{10} \sigma A^{2} \\
W_{m i x} & =\mathcal{A}\left(\operatorname{det}\left(S+A^{\prime \prime}+\sigma / \sqrt{10}\right)-B \bar{B}-\Lambda^{10}\right)+\frac{1}{2} \tilde{\lambda}_{5} \Lambda \sigma^{2}+\frac{1}{2} \tilde{\lambda}_{6} \Lambda S^{2}
\end{aligned}
$$

\footnotetext{
${ }^{4}$ This interesting feature is also used in Reference $[120,121]$ to give mass (in a different context) to some charged particles. This is accomplished by a cubic term in the superpotential that is a product of three different antisymmetric tensors.
} 


$$
\begin{aligned}
& +\frac{1}{2} \tilde{\lambda}_{7} \Lambda A^{\prime \prime 2}+\tilde{\lambda}_{16} \sigma \overline{\mathbf{1 6}} 16+\tilde{\lambda}_{4} A_{i j}^{\prime \prime} \overline{\mathbf{1 6}} \Sigma_{i j} \mathbf{1 6} \\
& +\tilde{\lambda}_{11}\left(A A^{\prime \prime}\right)_{i j} \overline{\mathbf{1 6}} \Sigma_{i j} \mathbf{1 6} / M .
\end{aligned}
$$

The naive expectation for the couplings is $\tilde{\lambda}_{2,3} \sim \lambda_{2} \Lambda / M, \tilde{\lambda}_{4} \sim \lambda_{3} \Lambda / M, \tilde{\lambda}_{16} \sim$ $\lambda_{4} \Lambda / M, \tilde{\lambda}_{5,6,7} \sim \lambda_{5} \Lambda / M, \tilde{\lambda}_{9,10} \sim \lambda_{7} \Lambda / M$, and $\tilde{\lambda}_{11} \sim \lambda_{11} \Lambda / M$.

It is assumed that $S, A^{\prime \prime}$, and $A$ acquire the vevs

$$
\begin{gathered}
S=s\left(1,1,1,-\frac{3}{2},-\frac{3}{2}\right) \otimes\left(\begin{array}{cc}
1 & 0 \\
0 & 1
\end{array}\right), A^{\prime \prime}=\left(a^{\prime \prime}, a^{\prime \prime}, a^{\prime \prime}, b^{\prime \prime}, b^{\prime \prime}\right) \otimes\left(\begin{array}{cc}
0 & -1 \\
& \\
1 & 0
\end{array}\right), \\
A=(a, a, a, b, b) \otimes\left(\begin{array}{cc}
0 & -1 \\
1 & 0
\end{array}\right) .
\end{gathered}
$$

These vevs break $S O(10) \rightarrow S U(3) \times S U(2) \times U(1)_{Y} \times U(1)_{X}$. The spinor field 16 is assumed to acquire a vev $\chi$ in the $S U(5)$-singlet direction ${ }^{5}$. The unbroken gauge group is then $S U(3) \times S U(2) \times U(1)_{Y}$. It is argued below that the superpotential guarantees that the vevs of $A, \sigma, A^{\prime \prime}, S, \mathbf{1 6}$ and $\mathcal{A}$ are naturally of the order of $\Lambda \sim M_{G U T}$ and $(\Lambda / M) \Lambda^{-7}$, respectively. Other vacua exist, but they are isolated from the vacuum considered here.

The doublets and triplets in $T_{1}$ are split using the DW mechanism [114]. The $F_{A}$ equations $\left(\tilde{\lambda}_{9} s+\tilde{\lambda}_{10} \sigma\right) a=0$ and $\left(-\frac{3}{2} \tilde{\lambda}_{9} s+\tilde{\lambda}_{10} \sigma\right) b=0$ with $s \neq 0$ forces either $a$ or $b$ to vanish; it is a discrete choice. The DW mechanism for giving the triplets in the $\boldsymbol{5}_{1,2}$ and $\overline{\boldsymbol{5}}_{1,2}$ Higgs fields GUT-sized masses requires that $b=0$. It is assumed that this minimum was selected in the early history of the universe. With this

\footnotetext{
${ }^{5}$ The $D$-flatness condition for $S O(10)$ requires the vevs of 16 and $\overline{\mathbf{1 6}}$ to be equal.
} 
choice, the mass matrix for the colored triplets in the $5_{1,2}$ and $\overline{\mathbf{5}}_{1,2}$ Higgs fields is

$$
\mathcal{M}=\left(\begin{array}{cc}
0 & -i \lambda_{1} a \\
i \lambda_{1} a & \tilde{\lambda}_{2} \sigma+\tilde{\lambda}_{3} s
\end{array}\right)
$$

in the $\left(T_{1}, T_{2}\right)$ basis. Since the diagonal element is suppressed by a factor of $O(\Lambda / M)$ relative to the off-diagonal element, the colored triplets form two Dirac particles with masses $M_{H_{C}} \sim \lambda_{1} a \sim \lambda_{1} \Lambda \sim \Lambda$. The mass matrix for the 4 electroweak doublets in $T_{1}$ and $T_{2}$ only has an entry for $T_{2}(2) T_{2}(\overline{2})$ since $b=0$. The mass of the Dirac heavy doublet is $\tilde{\lambda}_{2} \sigma-3 \tilde{\lambda}_{3} s / 2 \sim \Lambda^{2} / M$. The two electroweak doublets in $T_{1}$ are massless, and are identified as the Higgs fields responsible for giving mass to the up-type and down-type quarks of the SM.

It is interesting that the magnitude of the elements of $\mathcal{M}$ has a structure that is favorable for the suppression of the proton decay rate. It in fact provides a natural realization of the "weak suppression" of the decay rate that is advocated by Babu and Barr [120]. This is seen as follows. First note that the diagonal element is suppressed by a factor of $O(\Lambda / M)$ relative to the off-diagonal element, reflecting the fact that the diagonal entry arises from a non-renormalizable operator in the fundamental theory. If the SM fermions only couple to $T_{1}$, then the proton decay amplitude from the exchange of the heavy colored Higgsinos is proportional to $\mathcal{M}_{11}^{-1}$. In this case the matrix element is $\left(\tilde{\lambda}_{2} \sigma+\tilde{\lambda}_{3} s\right) /\left(\lambda_{1} a\right)^{2} \sim \Lambda^{2} / M$. This results in a decay rate that is approximately $(\Lambda / M)^{2} \sim 10^{-3}$ times the unsuppressed rate. This is sufficient to suppress the dangerous Higgsino-exchange proton decay operator to a level that may be observable at SuperKamiokande. To obtain the 
four-fermion operator responsible for the nucleon decay, the operator gotten by integrating out the colored triplet Higgsinos must be dressed with a vertex function involving either internal wino or gluino propagators. As emphasized in Reference [119], the gluino-dressed amplitude is comparable to the wino-dressed amplitude if $v_{u} / v_{d} \equiv \tan \beta$ is large. Since $\tan \beta \sim m_{t} / m_{b} \sim 40$ is naturally predicted within an $S O(10)$ GUT, the decay mode $\mathrm{p} \rightarrow K^{0} \mu^{+}$may be competitive with the (winodressed) neutrino decay modes [119].

The dominant decay modes for the wino-dressed operator are $\mathrm{p} \rightarrow K^{+} \bar{\nu}_{u}$ and $\mathrm{n} \rightarrow K^{0} \vec{\nu}_{u}$ [122]. To obtain an estimate for the nucleon lifetime in this model, their result for the lifetime of the nucleon is rescaled by a factor of $(M / \Lambda)^{2}$. The result is

$$
\tau\left(\mathrm{n} \rightarrow K^{0} \bar{\nu}_{u}\right) \sim 10^{32} \times\left(\frac{M}{31 \Lambda} \frac{0.0058 \mathrm{GeV}^{3}}{\beta} \frac{M_{H_{c}}}{10^{16} \mathrm{GeV}} \frac{\mathrm{TeV}^{-1}}{f(\tilde{u}, \tilde{d})+f(\tilde{u}, \tilde{e})}\right)^{2} \mathrm{yrs}
$$

The function $f$ is obtained by dressing the external squarks with wino propagators to obtain a four-fermion operator. It is computed in Reference [123], and depends on the sparticle spectrum. In the limit that the squark mass, $m_{\tilde{Q}}$, and slepton mass, $m_{\tilde{L}}$, are much larger than the wino mass, $m_{\tilde{w}}, f \sim r^{m}, m_{\tilde{X}}^{2}$, with $m_{\tilde{X}}$ the larger of $m_{\tilde{Q}}$ and $m_{\tilde{L}}$. The hadronic matrix element $\beta$ is defined in Reference [122]. Requiring that $M$ not exceed the Landau pole of the $S O(10)_{G U T}$ group implies that $M / \Lambda \lesssim 30-70$. (This constraint is discussed below.) This requirement of consistency also strongly constrains the presence of any additional matter content (this is also discussed below). This suggests that the Yukawa couplings of the 
SM fermions to the Higgs doublets are generated close to the GUT scale, a crucial assumption required to obtained the limit quoted in Eqn.(7.32). To obtain realistic quark and lepton masses in an $S O(10)$ model though, these Yukawa couplings probably arise from higher-dimensional operators [124]. In this case the flavor structure of the colored-triplet Higgs to matter may differ from the electroweak doublet couplings to matter, thereby altering the predicted lifetime [119]. For this reason, the result quoted in Eqn.(7.32) should be treated as an estimate. This estimate is to be compared with the existing experimental limit of $\tau(\mathrm{n} \rightarrow$ $\left.K^{0} \bar{\nu}_{u}\right)>.86 \times 10^{32}$ years [36]. So the nucleon lifetime is naturally suppressed to a phenomenologically interesting level.

Next the expected size of the vevs and the mass spectrum are discussed. The $F$-flatness equations are (setting $b=0$ )

$$
\begin{gathered}
\operatorname{det}\left(S+A^{\prime \prime}+\sigma / \sqrt{10}\right)-B \bar{B}=\Lambda^{10}, \\
\mathcal{A} B=0, \mathcal{A} \bar{B}=0 \\
0=F_{A}=\left(\tilde{\lambda}_{9} s+\tilde{\lambda}_{10} \sigma\right) a \\
0=F_{16}=\left(\tilde{\lambda}_{16} \sigma+\tilde{\lambda}_{4}\left(3 a^{\prime \prime}+2 b^{\prime \prime}\right)\right) \chi \\
0=F_{\sigma}=\tilde{\lambda}_{16} \chi^{2}-3 \tilde{\lambda}_{10} a^{2}+\tilde{\lambda}_{5} \Lambda \sigma+\frac{2}{\sqrt{10}} \mathcal{A} K(3 u+2 v), \\
0=F_{A^{\prime \prime}{ }_{3}}=\tilde{\lambda}_{4} \chi^{2}-2 \tilde{\lambda}_{7} \Lambda a^{\prime \prime}+2 \mathcal{A} K A, \\
0=F_{A^{\prime \prime}}=\tilde{\lambda}_{4} \chi^{2}-2 \tilde{\lambda}_{7} \Lambda b^{\prime \prime}+2 \mathcal{A} K B, \\
0=F_{S}=\tilde{\lambda}_{6} \Lambda s-\frac{2}{5}\left(\frac{1}{2} \tilde{\lambda}_{9} a^{2}-\mathcal{A} K(u-v)\right),
\end{gathered}
$$


where $K \equiv \operatorname{det}\left(S+A^{\prime \prime}+\sigma / \sqrt{10}\right)=\left(u^{2}+A^{2}\right)^{-3}\left(v^{2}+B^{2}\right)^{-2}$. The functions $u, v$, $A$ and $B$ are

$$
\begin{aligned}
& u=\frac{\sigma / \sqrt{10}+s}{(\sigma / \sqrt{10}+s)^{2}+a^{\prime 2}}, v=\frac{\sigma / \sqrt{10}-3 s / 2}{(\sigma / \sqrt{10}-3 s / 2)^{2}+b^{\prime 2}} \\
& A=\frac{a^{\prime \prime}}{(\sigma / \sqrt{10}+s)^{2}+a^{\prime \prime 2}}, B=\frac{b^{\prime \prime}}{(\sigma / \sqrt{10}-3 s / 2)^{2}+b^{\prime 2}}
\end{aligned}
$$

An inspection of these equations also indicates that without the operators $\Lambda S^{2}$, $\Lambda \sigma^{2}$ and $\Lambda A^{\prime \prime} A^{\prime \prime}$, the $F$-flatness equations would only constrain the values of $\mathcal{A}$, $\chi^{2}$ and $a^{2}$ in the combination $\chi^{2} / \mathcal{A}$ and $a^{2} / \mathcal{A}$. Thus one of these vevs would be unconstrained. As a result, not all the particle masses would be fixed by the input parameters. This problem is avoided by including the $(Q \bar{Q})^{2}$ operator, i.e. the operator $\propto \lambda_{5}$, in the fundamental theory. In this case, a new solution cannot be gotten by rescaling $\mathcal{A}$, with the $\tilde{\lambda}_{i}$ and $\Lambda$ fixed, and rescaling the vevs of any of the fields, thus indicating that $a^{2}, \chi^{2}$ and $\mathcal{A}$ are fixed by the input parameters.

Next it is argued that these equations fix the vevs of $S, A, \sigma$ and $A^{\prime \prime}$ to be on the order of $\Lambda$, without any fine tuning of the couplings in the fundamental theory. By redefining $\mathcal{A}=(\Lambda / M) \tilde{\mathcal{A}}$ the $F_{i}=0$ equations now contain an overall factor of $\Lambda / M$ if the expected relation between the superpotential couplings in the fundamental and confined theories is valid. As a result the $F_{i}$ equations no longer contain any small dimensionless couplings. The expected solution to this new set of equations is then $\chi, \sigma, a^{\prime \prime}, b^{\prime \prime}, a \sim s$ and $\tilde{\mathcal{A}} \sim \Lambda^{-7}$. The confinement equation fixes $s \sim \Lambda$. Therefore all the vevs are $v \sim \Lambda$ and $\mathcal{A} \sim(\Lambda / M) \Lambda^{-7}$. This result is not obvious a priori, since the superpotential couplings appearing 
in the $F$ equations are suppressed by powers of $\Lambda / M$. A slightly more rigorous argument, also showing that $\mathcal{A} \neq 0$, is presented in Appendix $\mathrm{D}$. This implies that the baryons vevs are forced to zero at this minimum. Two numerical solutions which support these arguments are also given in Appendix D. These expectations for the size of the couplings, $\mathcal{A}$, and vevs will be important below in estimating the mass spectrum.

The superpotential for this model contains enough operators to give superheavy masses to all the particles that should be heavy. The results of computing the mass matrices assuming a canonical Kähler potential are given in Appendix D, and are summarized here. The particles have masses at one of three scales: $m_{L} \equiv \Lambda^{4} / M^{3}$; $m_{I} \equiv \Lambda^{2} / M ;$ and $\Lambda$. The naive expectation is that all the particles have a mass $m \sim m_{I}$. This is because all the vevs are $O(\Lambda)$, and the mass matrices are linear in the superpotential couplings which contain a factor $\Lambda / M$, and in the parameter $\mathcal{A}$ which also contains a factor of $\Lambda / M$.

This expectation turns out to be correct except for a $u_{L} \sim(\overline{\mathbf{3}}, \mathbf{1},-2 / 3)$ and $\bar{u}_{L} \sim u_{L}^{\dagger}$, which acquire a Dirac mass from the superpotential operator $\left(A^{\prime \prime} A\right)_{i j} \overline{\mathbf{1 6}} \Sigma_{i j} \mathbf{1 6}$. These fields are massless in the absence of this operator for the following reason. The $S U(5)$ decomposition of $A=\mathbf{2 4}+\mathbf{1 0}+\overline{\mathbf{1 0}}+\mathbf{1}$. This clearly contains a $u \epsilon \mathbf{1 0}$ and $\bar{u} \epsilon \overline{\mathbf{1 0}}$. The only possible source for a mass term for these fields is given by $W_{D W}$. Further, since $S$ does not contain a $u$ and $\bar{u}$, this mass term must occur from setting $S$ and $\sigma$ to their vevs. The resulting mass is proportional to $\tilde{\lambda}_{9} s+\tilde{\lambda}_{10} \sigma$. The $D W$ form for $A$ and $F_{A}=0$, however, forces 
this quantity to vanish ${ }^{6}$. The addition of the operator $\operatorname{tr} A^{4} / M$ does not change the conclusion of this argument. The mass of these fields is gotten therefore from the $M^{-2}$ suppressed operator. The result of a computation of the mass spectrum, presented in Appendix D, implies that the naive expectation for their mass is $m \sim m_{L}$

The particle content of the fields with mass $m \sim m_{I}$ is now enumerated. The $S U(5)$ quantum numbers of the representations at this scale are: $1 \times(\mathbf{1 0}+\overline{\mathbf{5}})+$ $1 \times(\overline{\mathbf{1 0}}+5)+2 \times \mathbf{2 4}+1 \times(\mathbf{1 5}+\overline{\mathbf{1 5}})$. At the scale $m_{I}$ there is also a split ${ }^{7} \mathbf{2 4}$, with SM quantum numbers $8 \equiv(8, \mathbf{1}, 0)$ and $3 \equiv(\mathbf{1}, \mathbf{3}, 0)$. There are also some leftover fields, that together with $u_{L}$ and $\bar{u}_{L}$ which have a mass $m \sim m_{L}$, form a complete $\mathbf{1 0}+\overline{\mathbf{1 0}}$ of $S U(5)$. These leftover fields have a mass $m \sim m_{I}$. The representations in the $S O(10) \mathbf{1 0}_{\mathbf{1}}+\mathbf{1 0}_{\mathbf{2}}$ are split by the DW mechanism. One pair of electroweak doublets is massless and are the Higgs fields responsible for giving mass to the up-type quarks, down-type quarks, and leptons. The other doublet fields, $h \equiv(1,2,-1 / 2)$ and $\bar{h} \equiv(1,2,1 / 2)$, acquire a Dirac mass $m_{h} \sim m_{I}$. There are also a number of gauge singlets which acquire masses $m \sim m_{I}$.

The triplets in the $S O(10) \mathbf{1 0}_{\mathbf{1}}+\mathbf{1 0}_{\mathbf{2}}, 2 \times(\overline{\mathbf{3}}, \mathbf{1}, 1 / 3)+2 \times(\mathbf{3}, \mathbf{1},-1 / 3)$, acquire masses $O(\Lambda)$. The 33 Nambu-Goldstone bosons multiplets acquire a mass $m \sim \Lambda$ from the super-Higgs mechanism.

\footnotetext{
${ }^{6}$ The same argument also implies that the Majorana mass term for the 8 in $A$ vanishes. These fields, however, acquire a Dirac mass with the $8 \epsilon S$.

${ }^{7}$ The missing partners are the Nambu-Goldstone bosons of the $S U(5) \rightarrow S U(3)_{C} \times S U(2) \times$ $U(1)_{Y}$ breaking.
} 
The incomplete $S U(5)$ representations affect the prediction for $\sin ^{2} \theta_{W}$, which is discussed next. First it is approximated that all the charged particles at each of the three scales $m_{L}, m_{I}$ and $M_{G U T}$ are degenerate. In this approximation the contribution to $\Delta \sin ^{2} \theta_{W}$ occurs from splitting between the scales. The result of the usual one-loop computation implies that the light particles shift the prediction for $\sin ^{2} \theta_{W}$ by an amount

$$
\Delta \sin ^{2} \theta_{W}=-\frac{\alpha_{e m}}{2 \pi}\left(\ln \frac{m_{I}}{m_{L}}-\frac{4}{5} \ln \frac{\Lambda}{m_{I}}\right)
$$

The first term is the contribution from $u_{L}$ and $\bar{u}_{L}$; these fields only contribute between $m_{L}$ and $m_{I}$, since above the mass scale $m_{I}$ they fit into a complete $10+\overline{\mathbf{1 0}}$ of $S U(5)$. The second term is the sum of the contributions from 8 , 3, $h$ and $\bar{h}$. As is evident, for $m_{L}<m_{I}$ there is an $O(1)$ cancellation between the two contributions. Since $m_{L}$ arises from a higher dimensional operator than does $m_{I}, m_{L}<m_{I}$ applies for this model. It is then reasonable to expect that the $O(1)$ cancellation occurs. Inserting the naive expectation $m_{L} \sim \Lambda^{4} / M^{3}$ and $m_{I} \sim \Lambda^{2} / M$, gives

$$
\Delta \sin ^{2} \theta_{W} \sim-5 \times 10^{-3} \times \frac{\ln M / \Lambda}{\ln 30} .
$$

As is shown below, requiring that the $S O(10)_{G U T}$ not have a Landau pole below $M$ restricts $M / \Lambda \lesssim 30-70$. With this constraint, the shift in $\sin ^{2} \theta_{W}$ is consistent with the measured value, once other theoretical uncertainties are considered. The largest of these are uncalculable threshold corrections from the light (approximately) complete $S U(5)$ representations. Since the splitting within each multiplet 
gives a contribution that is naively $\alpha_{e m} / 2 \pi \times O(1)$, the large size of the light representations could result in a correction that is comparable or larger than the correction given in Eqn.(7.44).

It is next argued that any "gravitational smearing" [115] of the couplings at the GUT scale are small in this model. First, the only possible dimension-4 operator in the superpotential involving the $S O(10)_{G U T}$ chiral gauge multiplet $W_{i j}$ is $A_{i j} W_{j k} W_{k i} / M$. This, however, vanishes due to the anti-symmetry of $A$. Next, the operators $g_{S} S W W / M$ and $g_{\sigma} \sigma W W / M$ are allowed. The vev of $\sigma$ does not break $S U(5)$, so it only results in a common shift of the gauge couplings. The shift is tiny since $g_{\sigma} \sim \Lambda / M$. The vev of $S$ does break $S U(5)$, so this operator results in a tree-level correction to the unification of the couplings. An estimate for the shift in $\sin ^{2} \theta_{W}$ that this incurs is

$$
\Delta \sin ^{2} \theta_{W} \sim \pm 10^{-3} g_{S} \frac{30 s}{M} .
$$

It is expected that $g_{S} \sim \Lambda / M$ since this operator occurs from a dimension-4 operator in the superpotential of the fundamental theory. So this results in a tiny shift to $\sin ^{2} \theta_{\mathrm{I}}$. Finally, operators only involving $16, \overline{16}$ and $W W$ are also suppressed by an extra factor of $\Lambda / M$. The vev of $\mathbf{1 6}$ does not break $S U(5)$, so this operator only results in a tiny common shift to the gauge couplings.

An upper limit to $M$ is given by the value of the Landau pole of the $S O(10)$ GUT gauge coupling. This model is not asymptotically-free above the GUT scale since it contains a large particle content. More problematic though, is the fact 
that most of the particle masses are a factor of $\Lambda / M$ below the GUT scale. While this particle content does not result in a large shift to $\sin ^{2} \theta_{W}$ since they mostly form complete $S U(5)$ representations, the matter content does increase the value of $\alpha_{G U T}$. The value of $\alpha_{S 0(10)}(M)$, using naive one-loop running and with tree-level matching, and including the contribution of 316 s of the SM, is

$$
\alpha_{S O(10)}^{-1}(M)=24-\frac{3}{22 \pi}\left(\left(2+\frac{5}{3}\right) \ln \frac{\Lambda}{m_{L}}+\left(93-\frac{5}{3}\right) \ln \frac{\Lambda}{m_{I}}\right)-\frac{16}{2 \pi} \ln \frac{M}{\Lambda} .
$$

The second term is the contribution from $u_{L}+\bar{u}_{L}$, the third term is the contribution from the particles with mass $m_{I}$, and the last term is the contribution from the $S O(10)$ particle content above $\Lambda$. Inserting $m_{L} \sim \Lambda^{4} / M^{3}$ and $m_{I} \sim \Lambda^{2} / M$, the limit is

$$
\frac{M}{\Lambda} \lesssim 31
$$

This implies $M \sim 0.6-1 \times 10^{18} \mathrm{GeV}$. Note, however, that this limit is sensitive to the actual spectrum. For example, if the naive expectation underestimates the spectrum by a factor of 4 , then the limit increases to $M / \Lambda \lesssim 75$. This corresponds to $M \sim 1-2 \times 10^{18} \mathrm{GeV}$.

The Landau pole limit also strongly constrains any modifications to the model. For example, adding to the model either an extra adjoint $A^{\prime}$ which acquires a mass at $2 \times M_{G U T}$, or an extra $1 \mathbf{6}^{\prime}+\overline{\mathbf{1 6}}^{\prime}+\mathbf{1 0}^{\prime}+\mathbf{1 0 ^ { \prime \prime }}$ which all acquire a mass $M_{G U T}$ restricts $M / \Lambda \lesssim 20$. The presence of $N_{5}$ additional $S U(5) \mathbf{5}+\overline{\mathbf{5}}$ multiplets is also strongly constrained by this requirement of consistency. These fields would be required, for example, in any low-energy physics that is responsible for the origin 
of supersymmetry or flavor symmetry breaking. Requiring $M / \Lambda>20$ implies that the mass $M_{5}$ of these multiplets satisfies

$$
N_{5+\overline{5}} \ln M / M_{5} \lesssim 18
$$

In particular: $N_{5+\overline{5}}=1$ is marginally allowed if $M_{5}=10^{10} \mathrm{GeV} ; N_{5+\overline{5}}=2$ is marginally allowed if $M_{5}=10^{14} \mathrm{GeV}$. These constraints are weakened if the naive estimate, $\Lambda^{2} / M$, for the chiral GUT spectrum underestimates the spectrum by a factor of 4 . In this case,

$$
N_{5+\overline{5}} \ln M / M_{5} \lesssim 45
$$

for $M / \Lambda>20$. In particular: $N_{\mathbf{5}+\overline{\mathbf{5}}}=2$ is allowed for $M_{5}=10^{10} \mathrm{GeV} ; N_{\mathbf{5}+\overline{\mathbf{5}}}<5$ is required for $M_{5}=10^{14} \mathrm{GeV}$. Either direct or indirect evidence for additional chiral content that does not satisfy Eqn.(7.48) or Eqn.(7.49) would strongly disfavor this model.

This section concludes with a few comments about the consistency of neglecting certain operators in the superpotential. The superpotential terms $\sigma A_{i j} \overline{\mathbf{1 6}} \Sigma_{i j} \mathbf{1 6}$ or $S_{i k} A_{k j} \overline{\mathbf{1 6}} \Sigma_{i j} 16$ must be absent to avoid ruining the DW form for $A$. These operators would contribute to $F_{A}(2)$, forcing a non-vanishing value for $b$. These operators are present in the low-energy theory if the operators $\operatorname{tr}(\bar{Q} Q) A \overline{\mathbf{1 6}} \Sigma \mathbf{1 6}$ or $(\bar{Q} Q)_{S} A \overline{\mathbf{1 6}} \Sigma \mathbf{1 6}$ are present in the superpotential of the fundamental theory. Any symmetry which forbids these dangerous operators also forbids the operator $\left(A^{\prime \prime} A\right)_{i j} \overline{\mathbf{1 6}} \Sigma_{i j} \mathbf{1 6}$. This option is not viable since this cperator is required to give mass to a $(\overline{\mathbf{3}}, \mathbf{1},-2 / 3)+$ h.c. fields. (The DW form for $A$, however, is unaffected 
by the presence of this operator since it does not contribute to the $F_{i}$ equations.) So it must be assumed that the dangerous operators are not present in the fundamental theory. The perturbative non-renormalization theorems then guarantee that these operators will not be generated, at least in perturbation theory. This argument does not exclude the possibility that these dangerous operators could be generated by the non-perturbative dynamics of the $S U(10)_{S}$ or $S O(10)_{G U T}$ groups. By combining the requirement of holomorphy of the superpotential with some anomalous fake $U(1)$ symmetries [107] it is possible to exactly show, however, that if these operators are initially absent in the high-energy theory they will not be generated as the cutoff is lowered. In particular, it can be shown that the coefficient of a dangerous operator at a lower cutoff is only proportional to its initial value; i.e. it is independent of $\Lambda_{S U(10)} / M, \Lambda_{S O(10)} / M$ and all the other superpotential couplings. Then there is no reason for these dangerous operators to be generated by the confining dynamics.

\subsection{Summary}

In this chapter two models are presented that generate the Grand Unification scale from the strong dynamics of a confining group. The particle content of the confining group is chosen so that this sector confines with chiral symmetry breaking. The particles in this sector are also charged under the Grand Unified group. It follows that the composite fields which arise from the confining dynamics transform under the GUT group as either higher dimensional representations 
or singlets. Below the scale of confinement these composite fields acquire vevs. In each of the models presented here, there is a locally isolated supersymmetric vacuum in which the GUT group is broken to the SM group, and the resulting spectrum provides an acceptable phenomenology. Two GUT models are considered: $S U(6)$ and $S C(10)$. Known solutions to the doublet-triplet splitting problem are incorporated in each model. Proton decay in both models is at an acceptable rate, and in particular, in the $S O(10)$ model the dangerous dimension-5 proton decay operator is suppressed to an interesting level. This suppression is a natural consequence of the confining dynamics. Each model requires no fine tuning of any non-vanishing superpotential couplings. The fundamental theory in both models also contains an economical particle content, requiring no gauge singlets and only one higher dimensional representation. 


\section{Appendix A}

\section{Fine tuning Expressions}

In this section the Barbieri-Giudice parameters for both the MSSM and NMSSM in a gauge mediated SUSY breaking scenario are presented.

In an MSSM with gauge mediated SUSY breaking, the fundamental parameters of the theory (in the visible sector) are: $\Lambda_{\text {mess }} ; \lambda_{t} ; \mu$; and $\mu_{3}^{2}$. Once electroweak symmetry breaking occurs; the extremization conditions determine both $m_{Z}^{2}$ and $\tan \beta$ as a function of these parameters. To measure the sensitivity of $m_{Z}^{2}$ to one of the fundamental parameters $\lambda_{i}$, we compute the variation in $m_{Z}^{2}$ induced by a small change in one of the $\lambda_{i}$. The quantity

$$
\frac{\delta m_{Z}^{2}}{m_{Z}^{2}} \equiv c\left(m_{Z}^{2} ; \lambda_{i}\right) \frac{\delta \lambda_{i}}{\lambda_{i}}
$$

where

$$
c\left(m_{Z}^{2} ; \lambda_{i}\right)=\frac{\lambda_{i}}{m_{Z}^{2}} \frac{\partial m_{Z}^{2}}{\partial \lambda_{i}}
$$

measures this sensitivity [63]. In the case of gauge mediated SUSY breaking models, there are four functions $c\left(m_{Z}^{2} ; \lambda_{i}\right)$ to be computed. They are:

$$
\begin{gathered}
c\left(m_{Z}^{2} ; \mu^{2}\right)=\frac{2 \mu^{2}}{m_{Z}^{2}}\left(1+\frac{\tan ^{2} \beta+1}{\left(\tan ^{2} \beta-1\right)^{2}} \frac{4 \tan ^{2} \beta\left(\tilde{\mu}_{1}^{2}-\tilde{\mu}_{2}^{2}\right)}{\left.\left(\tilde{\mu}_{1}^{2}-\tilde{\mu}_{2}^{2}\right)\left(\tan ^{2} \beta+1\right)-m_{Z}^{2}\left(\tan ^{2} \beta-1\right)\right)}\right), \\
c\left(m_{Z}^{2} ; \mu_{3}^{2}\right)=4 \tan ^{2} \beta \frac{\tan ^{2} \beta+1}{\left(\tan ^{2} \beta-1\right)^{3}} \frac{\tilde{\mu}_{1}^{2}-\tilde{\mu}_{2}^{2}}{m_{Z}^{2}}
\end{gathered}
$$




$$
\begin{gathered}
\approx \frac{4}{\tan ^{2} \beta} \frac{\tilde{\mu}_{1}^{2}-\tilde{\mu}_{2}^{2}}{m_{Z}^{2}}, \text { for large } \tan \beta \\
c\left(m_{Z}^{2} ; \lambda_{t}\right)=2 \frac{\lambda_{t}^{2}}{m_{Z}^{2}} \frac{\partial m_{Z}^{2}}{\partial m_{H_{u}}^{2}} \frac{\partial m_{H_{u}}^{2}}{\partial \lambda_{t}^{2}} \\
=\frac{4}{m_{Z}^{2}} \lambda_{t}^{2} \frac{\tan ^{2} \beta}{\tan ^{2} \beta-1} \frac{\partial m_{H_{u}}^{2}}{\partial \lambda_{t}^{2}}\left(1+2 \frac{\tilde{\mu}_{1}^{2}-\tilde{\mu}_{2}^{2}}{\tilde{\mu}_{1}^{2}+\tilde{\mu}_{2}^{2}} \frac{\tan ^{2} \beta+1}{\left(\tan ^{2} \beta-1\right)^{2}}\right) \\
\approx \frac{4}{m_{Z}^{2}} \frac{\partial m_{H_{u}}^{2}}{\partial \lambda_{t}^{2}}, \text { for large } \tan \beta .
\end{gathered}
$$

This measures the sensitivity of $m_{Z}^{2}$ to the electroweak scale value of $\lambda_{t}, \lambda_{t}\left(M_{\text {weak }}\right)$. The Yukawa coupling $\lambda_{t}\left(M_{\text {weak }}\right)$ is not, however, a fundamental parameter of the theory. The fundamental parameter is the value of the coupling at the cutoff $\Lambda^{0}=M_{G U T}$ or $M_{p l}$ of the theory. We really should be computing the sensitivity of $m_{Z}^{2}$ to this value of $\lambda_{t}$. The measure of sensitivity is then correctly given by

$$
c\left(m_{Z}^{2} ; \lambda_{t}\left(\Lambda^{0}\right)\right)=\frac{\lambda_{t}\left(\Lambda^{0}\right)}{\lambda_{t}\left(M_{w e a k}\right)} c\left(m_{Z}^{2} ; \lambda_{t}\left(M_{w e a k}\right)\right) \frac{\partial \lambda_{t}\left(M_{w e a k}\right)}{\partial \lambda_{t}\left(\Lambda^{0}\right)}
$$

We remark that for the model discussed in the text with three $l+\bar{l}$ and one $q+\bar{q}$ messenger fields, the numerical value of $\left(\lambda_{t}\left(\Lambda^{0}\right) / \lambda_{t}\left(M_{w e a k}\right)\right) \partial \lambda_{t}\left(M_{\text {weak }}\right) / \partial \lambda_{t}\left(\Lambda^{0}\right)$ is typically $\sim 0.1$ because $\lambda_{t}\left(M_{w e a k}\right)$ is attracted to its infra-red fixed point. This results in a smaller value for $c\left(m_{Z}^{2} ; \lambda_{t}\right)$ than is obtained in the absence of these considerations.

With the assumption that $m_{H_{u}}^{2}$ and $m_{H_{d}}^{2}$ scale with $\Lambda_{m e s s}^{2}$, we get

$$
\begin{aligned}
c\left(m_{Z}^{2} ; \Lambda_{m e s s}^{2}\right)= & c\left(m_{Z}^{2} ; m_{H_{u}}^{2}\right)+c\left(m_{Z}^{2} ; m_{H_{d}}^{2}\right) \\
= & 1+2 \frac{\mu^{2}}{m_{Z}^{2}}-\frac{\tan ^{2} \beta+1}{\left(\tan ^{2} \beta-1\right)^{2}} \times \\
& \frac{4 \tan ^{2} \beta\left(m_{H_{u}}^{2}+m_{H_{d}}^{2}\right)\left(\tilde{\mu}_{1}^{2}-\tilde{\mu}_{2}^{2}\right) / m_{Z}^{2}}{\left(\tilde{\mu}_{1}^{2}-\tilde{\mu}_{2}^{2}\right)\left(\tan ^{2} \beta+1\right)-m_{Z}^{2}\left(\tan ^{2} \beta-1\right)} .
\end{aligned}
$$


The Barbieri-Giudice functions for $m_{t}$ are similarly computed. They are

$$
\begin{gathered}
c\left(m_{t} ; \mu_{3}^{2}\right)=\frac{1}{2} c\left(m_{Z}^{2} ; \mu_{3}^{2}\right)+\frac{1}{1-\tan ^{2} \beta}, \\
c\left(m_{t} ; \mu^{2}\right)=\frac{1}{2} c\left(m_{Z}^{2} ; \mu^{2}\right)+2 \frac{\mu^{2}}{\tilde{\mu}_{1}^{2}+\tilde{\mu}_{2}^{2}} \frac{1}{\tan ^{2} \beta-1}, \\
c\left(m_{t} ; \lambda_{t}\right)=1+\frac{1}{2} c\left(m_{Z}^{2} ; \lambda_{t}\right)+\frac{\lambda_{t}}{\tan ^{2} \beta-1} \frac{1}{\tilde{\mu}_{1}^{2}+\tilde{\mu}_{2}^{2}} \frac{\partial m_{H_{u}}^{2}}{\partial \lambda_{t}}, \\
c\left(m_{t} ; \Lambda_{m e s s}^{2}\right)=\frac{1}{2} c\left(m_{Z}^{2} ; \Lambda_{m e s s}^{2}\right)-\frac{\left(\tilde{\mu}_{1}^{2}+\tilde{\mu}_{2}^{2}-2 \mu^{2}\right)}{\left(1-\tan ^{2} \beta\right)\left(\tilde{\mu}_{2}^{2}+\tilde{\mu}_{1}^{2}\right)} .
\end{gathered}
$$

Since $m_{Z}$ and $m_{t}$ are measured, two of the four fundamental parameters may be eliminated. This leaves two free parameters, which for conveinence are chosen to be $\Lambda_{m e s s}$ and $\tan \beta$.

In a NMSSM with gauge mediated SUSY breaking, the scalar potential for $N, H_{u}$ and $H_{d}$ at the weak scale is specified by the following six parameters: $\lambda_{i}=m_{N}^{2}, m_{H_{u}}^{2}, m_{H_{d}}^{2}$, the $N H_{u} H_{d}$ coupling $\lambda_{H}$, the scalar $N H_{u} H_{d}$ coupling $A_{H}$, and the $N^{3}$ coupling, $\lambda_{N}$. In minimal gauge mediated SUSY breaking, the trilinear soft SUSY breaking term $\mathrm{NH}_{u} \mathrm{H}_{d}$ is zero at tree level and is generated at one loop by wino and bino exchange. In this case, $A_{H}\left(\lambda_{i}\right)=\lambda_{H} \tilde{A}\left(\lambda_{i}\right)$. Since the trilinear scalar term $N^{3}$ is generated at two loops, it is small and is neglected. The extremization conditions which determine $m_{Z}=g_{Z}^{2} v^{2} / 4\left(v=\sqrt{v_{u}^{2}+v_{d}^{2}}\right), \tan \beta=v_{u} / v_{d}$ and $v_{N}$ as a function of these parameters are given in Section 5.5. Eqn.(5.22) can be written, using $\mu=\lambda_{H} v_{N} / \sqrt{2}$ as

$$
m_{N}^{2}+2 \frac{\lambda_{N}^{2}}{\lambda_{H}^{2}} \mu^{2}-\lambda_{H} \lambda_{N} \frac{1}{2} v^{2} \sin 2 \beta+\frac{1}{2} \lambda_{H}^{2} v^{2}-\frac{1}{4 \mu} A_{H} v^{2} \lambda_{H} \sin 2 \beta=0
$$


Eqn.(5.23) is

$$
\frac{1}{8} g_{Z}^{2} v^{2}+\mu^{2}-m_{H_{u}}^{2} \frac{\tan ^{2} \beta}{1-\tan ^{2} \beta}+m_{H_{d}}^{2} \frac{1}{1-\tan ^{2} \beta}=0
$$

Substituting $v_{N}^{2}$ from Eqn.(5.22) in Eqn.(5.26) and then using this expression for $\mu_{3}^{2}$ in Eqn.(5.24) gives

$$
\left(m_{H_{u}}^{2}+m_{H_{d}}^{2}+2 \mu^{2}\right) \sin 2 \beta+\frac{\lambda_{H}}{\lambda_{N}}\left(m_{N}^{2}+\frac{1}{2} \lambda_{H}^{2} v^{2}\right)+A_{H}\left(-\frac{2 \mu}{\lambda_{H}}-\frac{1}{4} \frac{v^{2} \lambda_{H}^{2} \sin 2 \beta}{\mu \lambda_{N}}\right)=0 .
$$

The quantity $c=\left(\lambda_{i} / m_{Z}^{2}\right)\left(\partial m_{Z}^{2} / \partial \lambda_{i}\right)$ measures the sensitivity of $m_{Z}$ to these parameters. This can be computed by differentiating Eqns.(A.12), A.13 and A.14 with respect to these parameters to obtain, after some algebra, the following set of linear equations:

$$
\left(A+A_{A_{H}}\right) X^{i}=B^{i}+B_{A_{H}}^{i},
$$

where

$$
\begin{aligned}
& A=\left(\begin{array}{ccc}
\frac{1}{2} & 1 & \frac{\mu_{1}^{2}-\mu_{2}^{2}}{v^{2}} \frac{2 \tan \beta}{\left(1-\tan ^{2} \beta\right)^{2}} \\
\frac{\lambda_{H}^{3}\left(\lambda_{H}-\lambda_{N} \sin 2 \beta\right)}{g_{Z}^{2} \lambda_{N}^{2}} & 1 & -\frac{1}{2} \frac{\lambda_{H}^{3}}{\lambda_{N}} \frac{1-\tan ^{2} \beta}{\left(1+\tan ^{2} \beta\right)^{2}} \\
\frac{v^{2}}{a_{Z}^{2}\left(\mu_{1}^{2}+\mu_{2}^{2}\right)} \frac{\lambda_{H}^{3}}{\lambda_{N}} & \frac{\sin 2 \beta v^{2}}{\mu_{1}^{2}+\mu_{2}^{2}} & \frac{1-\tan ^{2} \beta}{\left(1+\tan ^{2} \beta\right)^{2}}
\end{array}\right) \\
& A_{A_{H}}=\frac{A_{H}}{\mu} \times \\
& \left(\begin{array}{ccc}
0 & 0 & 0 \\
-\frac{\lambda_{H}^{3} \sin 2 \beta}{2 g_{Z}^{2} \lambda_{N}^{2}} & \frac{\lambda_{H}^{3} \sin 2 \beta}{16 \lambda_{N}^{2}} \frac{v^{2}}{\mu^{2}} & \frac{\tan ^{2} \beta-1}{\left(1+\tan ^{2} \beta\right)^{2}} \frac{\lambda_{H}^{3}}{4 \lambda_{N}^{2}} \\
-\frac{\lambda_{H}^{2}}{2 g_{Z}^{2} \lambda_{N}} \frac{v^{2} \sin 2 \beta}{\mu_{1}^{2}+\mu_{2}^{2}} & \frac{v^{2}}{\mu_{1}^{2}+\mu_{2}^{2}}\left(\frac{\lambda_{H}^{2} \sin 2 \beta}{16 \lambda_{N}} \frac{v^{2}}{\mu^{2}}-\frac{1}{2 \lambda_{H}}\right) & \frac{\tan ^{2} \beta-1}{\left(1+\tan ^{2} \beta\right)^{2}} \frac{\lambda_{H}^{2}}{4 \lambda_{N}} \frac{v^{2}}{\mu_{1}^{2}+\mu_{2}^{2}}
\end{array}\right),
\end{aligned}
$$




$$
\begin{aligned}
X^{\lambda_{H}, \lambda_{N}} & =\left(\begin{array}{c}
\frac{1}{v^{2}} \frac{\partial m_{Z}^{2}}{\partial \lambda_{i}} \\
\frac{1}{v^{2}} \frac{\partial \mu^{2}}{\partial \lambda_{i}} \\
\frac{\partial \tan \beta}{\partial \lambda_{i}}
\end{array}\right), \\
X^{m_{i}^{2}} & =\left(\begin{array}{c}
\frac{\partial m_{Z}^{2}}{\partial m_{i}^{2}} \\
\frac{\partial \mu^{2}}{\partial m_{i}^{2}} \\
v^{2} \frac{\partial \tan \beta}{\partial m_{i}^{2}}
\end{array}\right),(i=u, d, N),
\end{aligned}
$$

with $\lambda_{i}=m_{N}^{2}, m_{H_{u}}^{2}, m_{H_{d}}^{2}, \lambda_{H}, \lambda_{N}$, and

$$
\begin{aligned}
& B^{m_{N}^{2}}+B_{A_{H}}^{m_{N}^{2}}=\left(\begin{array}{c}
0 \\
-\frac{1}{2} \frac{\lambda_{H}^{2}}{\lambda_{N}^{2}} \\
-\frac{\lambda_{H}}{\lambda_{N}} \frac{v^{2}}{2\left(\mu_{1}^{2}+\mu_{2}^{2}\right)}
\end{array}\right) \\
& B^{m_{H_{u}}^{2}+B_{A_{H}}^{m_{H_{u}}^{2}}}=\left(\begin{array}{c}
\frac{\tan ^{2} \beta}{1-\tan ^{2} \beta} \\
0 \\
-v^{2} \frac{\sin 2 \beta}{2\left(\mu_{1}^{2}+\mu_{2}^{2}\right)}
\end{array}\right) \text {, } \\
& B^{m_{H_{d}}^{2}+B_{A_{H}}^{m_{H_{d}}^{2}}}=\left(\begin{array}{c}
\frac{1}{\tan ^{2} \beta-1} \\
0 \\
-v^{2} \frac{\sin 2 \beta}{2\left(\mu_{1}^{2}+\mu_{2}^{2}\right)}
\end{array}\right) \text {, } \\
& B^{\lambda_{H}}=\left(\begin{array}{c}
0 \\
-\frac{\lambda_{H L}^{3}}{\lambda_{N}^{2}}+\frac{3}{4} \frac{\lambda_{H}^{2} \sin 2 \beta}{\lambda_{N}}-\frac{\lambda_{H}}{\lambda_{N}^{2}} \frac{m_{N}^{2}}{v^{2}} \\
-\frac{1}{\left(\mu_{1}^{2}+\mu_{2}^{2}\right)}\left(\frac{1}{2} \frac{m_{N}^{2}}{\lambda_{N}}+\frac{3}{4} v^{2} \frac{\lambda_{H}^{2}}{\lambda_{N}}\right)
\end{array}\right),
\end{aligned}
$$




$$
\begin{aligned}
B_{A_{H}}^{\lambda_{H}}= & \frac{A_{H}}{\mu}\left(\begin{array}{c}
0 \\
\frac{\lambda_{H}^{2} \sin 2 \beta}{2 \lambda_{N}^{2}} \\
\frac{3}{8} \frac{\lambda_{H}}{\lambda_{N}} \frac{v^{2} \sin 2 \beta}{\mu_{1}^{2}+\mu_{2}^{2}}
\end{array}\right) \\
B^{\lambda_{N}}= & \left(\begin{array}{c}
0 \\
-\frac{1}{4} \frac{\lambda_{H}^{3} \sin 2 \beta}{\lambda_{N}^{2}}+\frac{\lambda_{H}^{2}}{\lambda_{N}^{3}} \frac{m_{N}^{2}}{v^{2}}+\frac{1}{2} \frac{\lambda_{H}^{4}}{\lambda_{N}^{3}} \\
\frac{1}{2\left(\mu_{1}^{2}+\mu_{2}^{2}\right)} \frac{\lambda_{H}^{2}}{\lambda_{N}^{2}}\left(m_{N}^{2}+\frac{1}{2} v^{2} \lambda_{H}^{2}\right)
\end{array}\right) \\
B_{A_{H}}^{\lambda_{N}}= & \frac{A_{H}}{\mu}\left(\begin{array}{c}
0 \\
-\frac{\lambda_{H}^{3} \sin 2 \beta}{4 \lambda_{N}^{3}} \\
-\frac{\lambda_{H}^{2}}{8 \lambda_{N}^{2}} \frac{v^{2} \sin 2 \beta}{\mu_{1}^{2}+\mu_{2}^{2}}
\end{array}\right) .
\end{aligned}
$$

In deriving these equations $A_{H}\left(\lambda_{i}\right)=\lambda_{H} \tilde{A}\left(\lambda_{i}\right)$ was assumed and $\partial \tilde{A} / \partial \lambda_{H}$ was neglected. Inverting these set of equations gives the $c$ functions. We note that these expressions for the various $c$ functions are valid for any NMSSM in which the $N^{3}$ scalar term is negligible and the $N H_{u} H_{d}$ scalar term is proportional to $\lambda_{H}$. In general, these 6 parameters might, in turn, depend on some fundamental parameters, $\tilde{\lambda}_{i}$. Then, the sensitivity to these fundamental parameters is:

$$
\begin{aligned}
\tilde{c}_{i} & \equiv \frac{\tilde{\lambda}_{i}}{m_{Z}^{2}} \frac{\partial m_{Z}^{2}}{\partial \tilde{\lambda}_{i}} \\
& =\frac{\tilde{\lambda}_{i}}{m_{Z}^{2}} \sum_{j} \frac{\partial \lambda_{j}}{\partial \tilde{\lambda}_{i}} \frac{\partial m_{Z}^{2}}{\partial \lambda_{j}} \\
& =\sum_{j} \frac{\tilde{\lambda}_{i}}{\lambda_{j}} c\left(m_{Z}^{2} ; \lambda_{j}\right) \frac{\partial \lambda_{j}}{\partial \tilde{\lambda}_{i}} .
\end{aligned}
$$

For example, in the NMSSM of section 5.5, the fundamental parameters are $\Lambda_{\text {mess }}, \lambda_{H}, \lambda_{N}, \lambda_{t}$ and $\lambda_{q}\left(A_{H}\right.$ is a function of $\lambda_{H}$ and $\left.\Lambda_{\text {mess }}\right)$. Fixing $m_{Z}$ and 
$m_{t}$ leaves 3 free parameters, which we choose to be $\Lambda_{\text {mess }}, \lambda_{H}$ and $\tan \beta$. As explained in that section, the effect of $\lambda_{H}$ in the RG scaling of $m_{H_{u}}^{2}$ and $m_{H_{d}}^{2}$ was neglected, whereas the sensitivity of $m_{N}^{2}$ to $\lambda_{H}$ could be non-negligible. Thus, we have

$$
\tilde{c}\left(m_{Z}^{2} ; \lambda_{H}\right)=c\left(m_{Z}^{2} ; \lambda_{H}\right)+c\left(m_{Z}^{2} ; m_{N}^{2}\right) \frac{\lambda_{H}}{m_{N}^{2}} \frac{\partial m_{N}^{2}}{\partial \lambda_{H}} .
$$

We find, in our model, that $c\left(m_{Z}^{2} ; m_{N}^{2}\right)$ is smaller than $c\left(m_{Z}^{2} ; \lambda_{H}\right)$ by a factor of $\sim 2$. Also, using approximate analytic and also numerical solutions to the $R G$ equation for $m_{N}^{2}$, we find that $\left(\lambda_{H} / m_{N}^{2}\right)\left(\partial m_{N}^{2} / \partial \lambda_{H}\right)$ is $\lesssim 0.1$. Consequently, in the analysis of section 5.5 the additional contribution to $\tilde{c}\left(m_{Z}^{2} ; \lambda_{H}\right)$ due to the dependence of $m_{N}^{2}$ on $\lambda_{H}$ was neglected. A similar conclusion is true for $\lambda_{N}$. Also,

$$
\tilde{c}\left(m_{Z}^{2} ; \lambda_{q}\right)=c\left(m_{Z}^{2} ; m_{N}^{2}\right) \frac{\lambda_{q}}{m_{N}^{2}} \frac{\partial m_{N}^{2}}{\partial \lambda_{q}} .
$$

We find that $\left(\lambda_{q} / m_{N}^{2}\right)\left(\partial m_{N}^{2} / \partial \lambda_{q}\right)$ is $\approx 1$ so that $\tilde{c}\left(m_{Z}^{2} ; \lambda_{q}\right)$ is smaller than $\tilde{c}\left(m_{Z}^{2} ; \lambda_{H}\right)$ by a factor of 2 . 


\section{Appendix B}

\section{Two-loop calculation}

In this Section we discuss the two-loop contribution of the heavy scalar soft masses to the light scalar soft masses. These contributions can be divided into two classes. In the first class, a vev for the hypercharge $D$-term is generated at twoloops. The Feynman diagrams for these contributions are given in Figure B.1 and are clearly $\sim \alpha_{1} \alpha_{i}$. These diagrams are computed in a later portion of this Section. In the other class, the two-loop diagrams are $\sim \alpha_{i}^{2}$. These have been computed by Poppitz and Trivedi[106]. So, we will not give details of this computation which can be found in their paper. However, our result for the finite parts of these diagrams differs slightly from theirs and we discuss the reason for the discrepancy. When one regulates the theory using dimensional reduction $[101,102]$ (compactifying to $D<4$ dimensions), the vector field decomposes into a $D$-dimensional vector and $4-D$ scalars, called $\epsilon$-scalars, in the adjoint representation of the gauge group. Thus the number of Bose and Fermi degrees of freedom in the vector multiplet remain equal. The $\epsilon$-scalars receive, at one-loop, a divergent contribution to their mass, proportional to the supertrace of the mass matrix of the matter fields. Neglecting the fermion masses, this contribution is

$$
\delta m_{\epsilon}^{2}=-\frac{\alpha}{4 \pi}\left(\frac{2}{\epsilon}+\ln 4 \pi-\gamma\right)\left(n_{5}+3 n_{10}\right) M_{S}^{2}
$$


In our notation $D=4-\epsilon$. Poppitz and Trivedi choose the counterterm to cancel this divergence in the $M S$ scheme, i.e., the counterterm consists only of the divergent part, proportional to $1 / \epsilon$. When this counterterm is inserted in a oneloop $\epsilon$-scalar graph with SM fields (scalars) as the external lines, one obtains a divergent contribution to the SM scalar soft masses (the $1 / \epsilon$ of the counterterm is cancelled after summing over the $\epsilon$ adjoint scalars running in the loop). Poppitz and Trivedi use a cut-off, $\Lambda_{U V}$, to regulate this graph, giving a contribution from this graph that is:

$$
m_{i}^{2}=-\sum_{A}\left(n_{5}+3 n_{10}\right) C_{A}^{i} \frac{1}{16}\left(\frac{\alpha_{A}}{\pi}\right)^{2} M_{S}^{2} \ln \Lambda_{U V}^{2}
$$

with no finite part. We, on the other hand, choose the $\epsilon$-scalar mass counterterm in the $\overline{M S}$ scheme, i.e., proportional to $2 / \epsilon-\gamma+\ln 4 \pi$ (where $\gamma \approx 0.58$ is the Euler constant) and use dimensional reduction to regulate the graph with the insertion of the counterterm. This gives a contribution

$$
\begin{aligned}
m_{i}^{2} & =-\sum_{A}\left(n_{5}+3 n_{10}\right) C_{A}^{i} \frac{1}{16}\left(\frac{\alpha_{A}}{\pi}\right)^{2} M_{S}^{2}\left(\frac{2}{\epsilon}-\gamma+\ln 4 \pi\right)^{2} \epsilon \\
& =-\sum_{A}\left(n_{5}+3 n_{10}\right) C_{A}^{i} \frac{1}{8}\left(\frac{\alpha_{A}}{\pi}\right)^{2} M_{S}^{2}(2 / \epsilon-2 \gamma+2 \ln 4 \pi)
\end{aligned}
$$

In the first line the first factor of $(2 / \epsilon-\gamma+\ln 4 \pi)$ is from the counter-term insertion, the second factor is the result of the loop integral, and the over-all factor of $\epsilon$ counts the number of $\epsilon$-scalars running in the loop. In the $\overline{M S}$ scheme, i.e., after subtracting $2 / \epsilon-\gamma+\ln 4 \pi$, we are left with a finite $\operatorname{part}^{1}$ proportional to $-\gamma+\ln 4 \pi$. The remaining diagrams together give a finite result and we agree with Poppitz

\footnotetext{
${ }^{1}$ The same finite part is obtained in the $M S$ scheme, regulated with $D R^{\prime}$.
} 

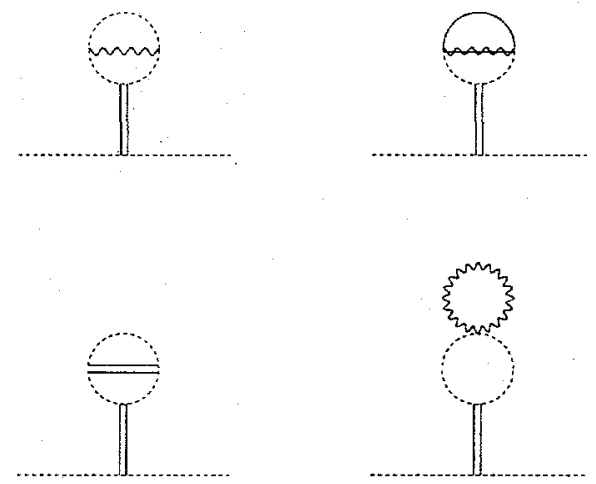

Figure B.1: Mixed two-loop corrections to the scalar mass. Wavy lines, wavy lines with a straight line through them, solid lines, and dashed lines denote gauge boson, gaugino, fermion and scalar propagators, respectively. The double-line denotes the hypercharge $D$-term propagator.

and Trivedi on this computation. Our result for the finite part of the two-loop diagrams (neglecting the fermion masses) is

$$
\begin{aligned}
m_{i, \text { finite }}^{2}(\mu)= & -\frac{1}{8}\left(\ln (4 \pi)-\gamma+\frac{\pi^{2}}{3}-2-\ln \left(\frac{M_{S}^{2}}{\mu^{2}}\right)\right) \\
& \times \sum_{A}\left(\frac{\alpha_{A}(\mu)}{\pi}\right)^{2}\left(n_{5}+3 n_{10}\right) C_{A}^{i} M_{S}^{2}
\end{aligned}
$$

whereas the Poppitz-Trivedi result does not have the $\ln (4 \pi)-\gamma$ in the above result. The computation of the two-loop hypercharge $D$-term, which gives contribution to the soft scalar mass squareds proportional to $\alpha_{1} \alpha_{s}$ and $\alpha_{1} \alpha_{2}$ (i.e., the "mixed" two-loop contributon) is discussed below in detail.

Two-loop hypercharge D-term 
The two-loop diagrams of Figure B.1 are computed in the Feynman gauge and all the fermion and gaugino masses are set to zero. It is convenient to calculate in this gauge because both the scalar self-energy and the $D_{Y}$-term vertex corrections are finite at one-loop and thus require no counter-terms. We have also computed the two-loop diagrams in the Landau gauge and have found that it agrees with the calculation in the Feynman gauge. The calculation in the Landau gauge requires counter-terms, is more involved, and hence the discussion is not included. Finally, in the calculation a global $S U(5)$ symmetry is assumed so that a hypercharge $D$ term is not generated at one-loop [96, 92].

The sum of the four Feynman diagrams in Figure B.1 is given in the Feynman gauge by

$$
-i \tilde{\Pi}_{D, f}=i \frac{3}{5} g_{1}^{2} Y_{f} \operatorname{Tr} Y_{i} \sum_{A, i} g_{A}^{2} C_{A}^{i}\left(4 I_{1}\left(m_{i}^{2}\right)-4 I_{2}\left(m_{i}^{2}\right)+I_{3}\left(m_{i}^{2}\right)\right)
$$

where the trace is over the gauge and flavour states of the particles in the loops. If the particles in the loop form complete $\overline{5}$ and 10 representations with a common mass $M_{S}$, the sum simplifies to

$$
-i \tilde{\Pi}_{D, f}=i \frac{3}{5} \alpha_{1} Y_{f}\left(n_{5}-n_{10}\right)\left(\frac{4}{3} \alpha_{3}-\frac{3}{4} \alpha_{2}-\frac{1}{12} \alpha_{1}\right)\left(4 I_{1}\left(M_{S}^{2}\right)-4 I_{2}\left(M_{S}^{2}\right)+I_{3}\left(M_{S}^{2}\right)\right) .
$$

The functions $I_{1}, I_{2}$ and $I_{3}$ are

$$
\begin{aligned}
& I_{1}\left(m^{2}\right)=\int \frac{d^{D} p}{(2 \pi)^{D}} \int \frac{d^{D} k}{(2 \pi)^{D}} \frac{1}{\left(p^{2}-m^{2}\right)^{2}} \frac{(2 p-k)^{2}}{k^{2}} \frac{1}{(p-k)^{2}-m^{2}} \\
& I_{2}\left(m^{2}\right)=\int \frac{d^{D} p}{(2 \pi)^{D}} \int \frac{d^{D} k}{(2 \pi)^{D}} \frac{1}{\left(p^{2}-m^{2}\right)^{2}} \frac{k^{2}-k \cdot p}{k^{2}} \frac{1}{(p-k)^{2}} \\
& I_{3}\left(m^{2}\right)=\int \frac{d^{D} k}{(2 \pi)^{D}} \frac{1}{\left(k^{2}-m^{2}\right)^{2}} \int \frac{d^{D} q}{(2 \pi)^{D}} \frac{1}{q^{2}-m^{2}} .
\end{aligned}
$$


These functions are now computed.

\section{$\underline{\text { Evaluating } I_{1}}$}

After a Feynman parameterization and performing a change of variables, $I_{1}=$ $J_{1}+J_{2}$, where

$$
J_{1}\left(m^{2}\right)=\Gamma(3) \int_{0}^{1} d x(1-x) \int \frac{d^{D} p}{(2 \pi)^{D}} \int \frac{d^{D} k}{(2 \pi)^{D}} 4 \frac{p^{2}}{k^{2}} \frac{1}{\left(p^{2}-\left(m^{2}-x(1-x) k^{2}\right)\right)^{3}}
$$

and

$$
J_{2}\left(m^{2}\right)=\Gamma(3) \int_{0}^{1} d x(1-x)(2 x-1)^{2} \int \frac{d^{D} p}{(2 \pi)^{D}} \int \frac{d^{D} k}{(2 \pi)^{D}} \frac{1}{\left(p^{2}-\left(m^{2}-x(1-x) k^{2}\right)\right)^{3}}
$$

After some algebra we find that

$$
\begin{gathered}
J_{1}\left(m^{2}\right)=\frac{\Gamma(3-D)}{(4 \pi)^{D}}\left(m^{2}\right)^{D-3} \frac{2 D}{D / 2-1} B(2-D / 2,3-D / 2), \\
J_{2}\left(m^{2}\right)=\frac{\Gamma(3-D)}{(4 \pi)^{D}}\left(m^{2}\right)^{D-3} \times(4 B(3-D / 2,2-D / 2)-4 B(2-D / 2,2-D / 2)+B(1-D / 2,2-D / 2)),
\end{gathered}
$$

where $B(p, q)=\Gamma(p) \Gamma(q) / \Gamma(p+q)$ is the usual Beta function.

Combining these two results gives

$$
I_{1}\left(m^{2}\right)=\frac{\Gamma(3-D)}{(4 \pi)^{D}}\left(m^{2}\right)^{D-3} \frac{1-D}{D-2} B(3-D / 2,2-D / 2) .
$$

\section{$\underline{\text { Evaluating } I_{2}}$}

$$
\begin{aligned}
I_{2}\left(m^{2}\right) & =\int \frac{d^{D} p}{(2 \pi)^{D}} \int \frac{d^{D} k}{(2 \pi)^{D}} \frac{1}{\left(p^{2}-m^{2}\right)^{2}} \frac{k^{2}-k \cdot p}{k^{2}} \frac{1}{(p-k)^{2}} \\
& =\frac{1}{(4 \pi)^{D}} \Gamma(3-D)\left(m^{2}\right)^{D-3} B(D / 2,1-D / 2) .
\end{aligned}
$$




\section{$\underline{\text { Evaluating } I_{3}}$}

$$
\begin{aligned}
I_{3}\left(m^{2}\right) & =\int \frac{d^{D} k}{(2 \pi)^{D}} \frac{1}{\left(k^{2}-m^{2}\right)^{2}} \int \frac{d^{D} q}{(2 \pi)^{D}} \frac{1}{q^{2}-m^{2}} \\
& =\left(\frac{i}{(4 \pi)^{D / 2}} \Gamma(2-D / 2)\left(m^{2}\right)^{D / 2-2}\right)\left(\frac{i}{(4 \pi)^{D / 2}} \frac{\Gamma(2-D / 2)}{D / 2-1}\left(m^{2}\right)^{D / 2-1}\right) \\
& =-\frac{1}{(4 \pi)^{D}}(\Gamma(2-D / 2))^{2} \frac{1}{D / 2-1}\left(m^{2}\right)^{D-3}
\end{aligned}
$$

We may now combine $I_{1}, I_{2}$ and $I_{3}$ to obtain

$$
\begin{aligned}
T\left(m^{2}\right) \equiv & 4 I_{1}\left(m^{2}\right)-4 I_{2}\left(m^{2}\right)+I_{3}\left(m^{2}\right) \\
= & \frac{\left(m^{2}\right)^{D-3}}{(4 \pi)^{D}} \times\left(4\left(\frac{1-D}{D-2} B(3-D / 2,2-D / 2)-B(D / 2,1-D / 2)\right) \Gamma(3-D)\right. \\
& \left.-\frac{1}{D / 2-1} \Gamma(2-D / 2)^{2}\right) .
\end{aligned}
$$

Writing $D=4-\epsilon$ and expanding in $\epsilon$ gives

$$
T\left(m^{2}\right)=\frac{1}{\left(16 \pi^{2}\right)^{2}}\left(\frac{4}{\epsilon}+\left(6-\frac{2}{3} \pi^{2}+4(\ln (4 \pi)-\gamma)-4 \ln m^{2}\right) m^{2}+O(\epsilon)\right) .
$$

In the $\overline{M S}$ scheme the combination $2 / \epsilon+\ln (4 \pi)-\gamma$ is subtracted out. The finite piece that remains is

$$
\frac{1}{\left(16 \pi^{2}\right)^{2}}\left(6-\frac{2}{3} \pi^{2}+2(\ln (4 \pi)-\gamma)-4 \ln m^{2}\right) m^{2}
$$

Thus in the $\overline{M S}$ scheme

$$
\begin{aligned}
-i \tilde{\Pi}_{D, f}= & i \frac{3}{5} \frac{1}{\left(16 \pi^{2}\right)^{2}} g_{1}^{2} Y_{f} \operatorname{Tr}_{i} Y_{i} \sum_{A} g_{A}^{2} C_{A}^{i}\left(6-\frac{2}{3} \pi^{2}+2(\ln (4 \pi)-\gamma)-4 \ln m_{i}^{2}\right) m_{i}^{2} \\
= & i \frac{3}{5} \frac{1}{16 \pi^{2}} \alpha_{1}\left(\mu_{R}\right)\left(n_{5}-n_{10}\right) Y_{f}\left(6-\frac{2}{3} \pi^{2}+2(\ln (4 \pi)-\gamma)-4 \ln \left(\frac{M_{S}^{2}}{\mu_{R}^{2}}\right)\right) \\
& \times\left(\frac{4}{3} \alpha_{3}\left(\mu_{R}\right)-\frac{3}{4} \alpha_{2}\left(\mu_{R}\right)-\frac{1}{12} \alpha_{1}\left(\mu_{R}\right)\right) M_{S}^{2}
\end{aligned}
$$




\section{Appendix C}

\section{Spectrum of $S U(6) \times S U(6)_{G U T}$}

First the existence of a solution to the $F_{i}=0$ equations with all vevs of $O(\Lambda)$ and $\mathcal{A} \sim(\Lambda / M) \Lambda^{-3}$ is discussed. The second part of this Appendix contains the results of calculating the mass spectrum, assuming a canonical Kähler potential.

Since the $F_{i}=0$ equations are linear in $v_{H}^{2}$ and $v_{N}^{2}$, it is straightforward to solve for them in terms of $\sigma$ and $v_{\Sigma}$. The remaining two equations determine $\mathcal{A} \neq 0$ and $x \equiv \sigma / v_{\Sigma}$. In particular, $x$ is the solution to

$$
\beta x^{2}-(\sqrt{6} \beta-\alpha-\gamma) x-\sqrt{6} \alpha+12 \beta=0
$$

where $\alpha \equiv-\tilde{\lambda}_{1}, \beta \equiv-\tilde{\lambda}_{3} \tilde{\lambda}_{4} M /(12 g \Lambda)$, and $\gamma \equiv-\tilde{\lambda}_{2}-24 \tilde{\lambda}_{5} \beta / \tilde{\lambda}_{4}$. Note that $\beta \sim \alpha \sim \gamma \sim \Lambda / M$. Since each term appearing in Eqn.(C.1) is linear in $\Lambda / M$, it follows that $x \sim O(1)$, i.e. $\sigma \sim v_{\Sigma}$ is expected. The quantum constraint then fixes $v_{\Sigma} \sim \Lambda$. It follows from $F_{H}=0$ that $v_{N}^{2}=-\tilde{\lambda}_{3} M \sigma /(12 g)$ is $O\left(\Lambda^{2}\right)$. Next, $v_{H}^{2}=-\left(M v_{\Sigma} / g\right)\left(\tilde{\lambda}_{5} x-\tilde{\lambda}_{4}\right)$ is also $O\left(\Lambda^{2}\right)$. Finally, either $F_{\Sigma}=0$ or $F_{\sigma}=0$ determines $\mathcal{A} \sim(\Lambda / M) \Lambda^{-3}$.

The non-Nambu-Goldstone multiplet fields charged under the SM, with the exception of the SM Higgs doublets, are all contained in $\Sigma$ and $\Sigma_{N}$. Since these fields acquire their mass from the $S U(4) \times S U(2)$ preserving vevs of $\Sigma, \Sigma_{N}$ or $(H \bar{H})$, it is convenient to classify the mass spectrum according to the $S U(4) \times S U(2)$, 
rather than the $S U(3) \times S U(2) \times U(1)_{Y}$, charge assignments.

The mass matrix for the $Q \sim(4,2)$ and $\bar{Q} \sim(\overline{4}, 2)$ fields (after some algebra using the $F_{i}=0$ equations) in the $\left(\Sigma, \Sigma_{N}\right)$ basis is

$$
M_{\bar{Q} Q}=\left(\begin{array}{cc}
-\mathcal{A} K a b+\tilde{\lambda}_{1} \Lambda & -\tilde{\lambda}_{4} v_{N} \\
-\tilde{\lambda}_{4} v_{N} & \tilde{\lambda}_{4} v_{\Sigma}
\end{array}\right) .
$$

By using the $F_{i}=0$ equations it can be verified that this matrix annihilates the state $\left(v_{\Sigma}, v_{N}\right)$, which is a Nambu-Goldstone boson of the gauge symmetry breaking. The massive eigenvalue is non-zero and naively $m_{Q} \sim \Lambda^{2} / M$.

The mass matrix for the $(\mathbf{1 5}, \mathbf{1})$ fields (after some algebra using the $F_{i}=0$ equations) in the $\left(\Sigma, \Sigma_{N}\right)$ basis is

$$
M_{15}=\left(\begin{array}{cc}
-\mathcal{A} K a^{2}+\tilde{\lambda}_{1} \Lambda & 2 \tilde{\lambda}_{4} v_{N} \\
2 \tilde{\lambda}_{4} v_{N} & 4 \tilde{\lambda}_{4} v_{\Sigma}
\end{array}\right) .
$$

It can be shown after some algebra that the determinant of this matrix is $-4 \tilde{\lambda}_{4} \mathcal{A} K a v_{\Sigma}(a-b)$. This is non-zero since $v_{\Sigma} \neq 0$ implies that $a \neq b$. The expected masses for the two eigenvalues is then $m_{15} \sim \Lambda^{2} / M$.

The mass matrix for the $(\mathbf{1}, \mathbf{3})$ fields (after some algebra using the $F_{i}=0$ equations) in the $\left(\Sigma, \Sigma_{N}\right)$ basis is

$$
M_{3}=\left(\begin{array}{cc}
-\mathcal{A} K b^{2}+\tilde{\lambda}_{1} \Lambda & -4 \tilde{\lambda}_{4} v_{N} \\
-4 \tilde{\lambda}_{4} v_{N} & -2 \tilde{\lambda}_{4} v_{N}
\end{array}\right) .
$$

It can be shown that the determinant of this matrix is $-\sqrt{6} \tilde{\lambda}_{4} \Lambda b v_{\Sigma}^{2}\left(3 \beta x^{2}-5 \beta \sqrt{6} x+\right.$ $\sqrt{6} \alpha$ ). A comparison of this result with Eqn.(C.1) indicates that it is non-vanishing 
for generic values of the $\tilde{\lambda}_{i}$ s. The expected masses for the two eigenvalues is then $m_{3} \sim \Lambda^{2} / M$. 


\section{Appendix D}

\section{Spectrum of $S U(10) \times S O(10)_{G U T}$ Model}

\section{$\underline{\text { Arguing that all the vevs are of order } \Lambda ; \underline{\text { Numerical solution }}}$}

In this the only concern is whether a discrete solution with all $\mathcal{A}, a, a^{\prime \prime}, b^{\prime \prime}$, $\sigma, s$ and $\chi$ non-zero exists. This result is obtained by showing that if $s \neq 0$, then $\mathcal{A} \neq 0$ and all other vevs are comparable to $s$. Then the non-vanishing of $\mathcal{A}$ implies that $B=\bar{B}=0$. The confinement condition then fixes $s \sim \Lambda$. To begin, first note that $F_{A}$ fixes $\sigma \sim s$. The $F_{16}$ equation implies that $3 a^{\prime \prime}+2 b^{\prime \prime} \sim \sigma \sim s$. Thus either $a^{\prime \prime} \sim b^{\prime \prime} \sim s$, or $b^{\prime \prime} \ll a^{\prime \prime} \sim s$ (or $a^{\prime \prime} \ll b^{\prime \prime} \sim s$ ). Next it is argued that the last two cases do not occur. In the first case, $b^{\prime \prime} \ll a^{\prime \prime}$, so that $B \ll A$. Next, the two $F_{A^{\prime \prime}}$ equations are inconsistent if either $\mathcal{A} K A \ll \tilde{\lambda}_{7} a^{\prime \prime}$ or $\mathcal{A} K A \gg \tilde{\lambda}_{7} a^{\prime \prime}$. So $\mathcal{A} K A \sim \tilde{\lambda}_{7} a^{\prime \prime}$ and $\tilde{\lambda}_{7} b^{\prime \prime} \sim \chi^{2} \ll \tilde{\lambda}_{7} a^{\prime \prime}$ is the only consistent solution to the two $F_{A^{\prime \prime}}$ equations. Thus if $b^{\prime \prime} \ll a^{\prime \prime}, F_{16}$ fixes $a^{\prime \prime} \sim s$ up to small corrections of $O\left(b^{\prime \prime}\right)$. Similarly, the first $F_{A^{\prime \prime}}$ fixes $\mathcal{A}$ up to small corrections. But now the two equations $F_{\sigma}$ and $F_{S}$ each determine $a \sim s$; these two equations for $a$ cannot in general be simultaneously satisfied. Therefore, $b^{\prime \prime} \ll a^{\prime \prime}$ is not a viable (supersymmetric) solution. The argument against $a^{\prime \prime} \ll b^{\prime \prime}$ is similar. Therefore $a^{\prime \prime} \sim b^{\prime \prime}$. Next suppose that $\mathcal{A}=0$. Then $F_{A^{\prime \prime}}$ fixes $a^{\prime \prime}=b^{\prime \prime}$, and together with $F_{16}$ and $F_{A}$, determines $\chi \sim s$. But now there are two remaining equations, $F_{S}$ and $F_{\sigma}$, for one 
unknown, $a$. More concretely, $a^{2}=5\left(\tilde{\lambda}_{6} / \tilde{\lambda}_{9}\right) \Lambda s$ and $a^{2}=\left(\tilde{\lambda}_{5}-\frac{2}{5} \tilde{\lambda}_{7} \tilde{\lambda}_{16}^{2} / \tilde{\lambda}_{4}^{2}\right) \Lambda \sigma / 3 \tilde{\lambda}_{10}$. In general, these two equations will not be satisfied; therefore $\mathcal{A} \neq 0$. The vev $a$ can be eliminated from $F_{S}$ and $F_{\sigma}$; the remaining equation, together with $F_{A^{\prime \prime}}$ and $F_{16}$ may be used in principle to determine $\chi, a^{\prime \prime}, b^{\prime \prime} \sim s$ and also fix $\mathcal{A} .\left(\chi^{2} \ll \Lambda a^{\prime \prime}\right.$ is not possible; $F_{A}, F_{S}, F_{A^{\prime \prime}}, F_{16}$ and $F_{\sigma}$ are 6 equations in only 5 unknowns: $\sigma$, $a^{\prime \prime}, b^{\prime \prime}, a$ and $\mathcal{A}$.) The $F_{S}$ equation will not in general be satisfied with $a^{2} \ll \Lambda s$ or $a^{2} \gg \Lambda s$; since $\mathcal{A} K(u-v)$ is $O\left(\Lambda^{2} s / M\right)$ and $\neq \tilde{\lambda}_{6} \Lambda s$ in general, $F_{S}$ determines $a \sim s$.

Two numerical solutions to the $F_{i}=0$ equations supports these arguments. In the first (I) solution, the input parameters are chosen to be : $\tilde{\lambda}_{4}=0.01, \tilde{\lambda}_{5}=0.02$, $\tilde{\lambda}_{6}=0.03, \tilde{\lambda}_{7}=0.04, \tilde{\lambda}_{9}=0.05, \tilde{\lambda}_{10}=0.06$ and $\tilde{\lambda}_{16}=0.045$. The solution, in units of $\Lambda=1$, is

$$
\sigma=-0.64, s=0.77, a^{\prime \prime}=0.50, b^{\prime \prime}=0.70, a=1.2, \chi=2.5, \mathcal{A}=-0.01
$$

In the second (II) solution, the input parameters are chosen to be : $\tilde{\lambda}_{4}=0.0134$, $\tilde{\lambda}_{5}=0.0123, \tilde{\lambda}_{6}=-0.03, \tilde{\lambda}_{7}=0.0225, \tilde{\lambda}_{9}=0.045, \tilde{\lambda}_{10}=0.0623$ and $\tilde{\lambda}_{16}=$ 0.03657 . The solution, in units of $\Lambda=1$, is

$$
\sigma=-0.62, s=0.85, a^{\prime \prime}=-0.14, b^{\prime \prime}=1.1, a=-0.87, \chi=1.2, \mathcal{A}=0.04 \text {. }
$$

These parameters are chosen to be small since $\tilde{\lambda} \sim \lambda \Lambda / M \sim 0.03 \lambda$ for $\Lambda / M \sim$ $1 / 30$. Aside from this feature, there is nothing special about this choice of super- 
potential couplings. As expected, all the vevs are $O(\Lambda)$ and $\mathcal{A} \sim(\Lambda / M) \Lambda^{-7}$.

\section{Detailed Mass Spectrum}

The mass matrices presented here were computed assuming a canonical Kähler potential; this is suffucient to determine the rank of the matrix.

For future purposes it will be useful to note that the $F_{i}$ equations are invariant under the following rescaling of couplings and fields:

$$
\begin{gathered}
\left(\tilde{\lambda}_{4}, \tilde{\lambda}_{9}, \tilde{\lambda}_{10}, \tilde{\lambda}_{16}\right) \rightarrow\left(g^{-2} \tilde{\lambda}_{4}, g^{-2} \tilde{\lambda}_{9}, g^{-2} \tilde{\lambda}_{10}, g^{-2} \tilde{\lambda}_{16}\right) \\
(\chi, a) \rightarrow(g \chi, g a),\left(a^{\prime \prime}, b^{\prime \prime}, s, \sigma, K\right) \rightarrow\left(a^{\prime \prime}, b^{\prime \prime}, s, \sigma, K\right) .
\end{gathered}
$$

Any coupling not listed is left invariant. This mapping relates the solutions to the $F_{i}=0$ equations in two theories with different superpotential couplings which are related by this scale transformation.

The $u^{c} \sim(\overline{\mathbf{3}}, 1,-2 / 3)+$ h.c. mass matrix in the $\left(A^{\prime \prime}, \mathbf{1 6}(\overline{\mathbf{1 6}}), A\right)$ basis is, with $\bar{\lambda} \equiv \tilde{\lambda}_{11} / M$

$$
M_{u^{c}, \bar{u}^{c}}=\left(\begin{array}{ccc}
2 \mathcal{A} K\left(u^{2}+A^{2}\right)-2 \tilde{\lambda}_{7} \Lambda & 2 i \tilde{\lambda}_{4} \chi-2 \bar{\lambda} a \chi & i \bar{\lambda} \chi^{2} \\
-2 i \tilde{\lambda}_{4} \chi-2 \bar{\lambda} a \chi & -4 \tilde{\lambda}_{4} a^{\prime \prime} & -2 \bar{\lambda} a^{\prime \prime} \chi \\
-i \bar{\lambda} \chi^{2} & -2 \bar{\lambda} a^{\prime \prime} \chi & 0
\end{array}\right)
$$

Using the $F_{i}=0$ equations the reader can verify that this matrix has only one zero eigenvalue. The product of the two non-zero eigenvalues is given by the coefficient of $O(e)$ in the expansion of $\operatorname{det}\left(M_{u}-e \mathbf{1}\right)$. This coefficient is $\bar{\lambda}^{2} \chi^{2}\left(4 a^{2}+4 a^{\prime \prime 2}+\chi^{2}\right)$. Therefore, this matrix contains an extra massless particle in the limit $\bar{\lambda} \rightarrow 0$. With $\bar{\lambda} \neq 0$, the naive expectation for this product of eigenvalues is $(\Lambda / M)^{4} \Lambda^{2}$. The 
larger eigenvalue is $m_{u_{H}}=\tilde{\lambda}_{4}\left(4 a^{\prime \prime}+\chi^{2} / a^{\prime \prime}\right)$, and is approximately $\Lambda^{2} / M$. So the smaller eigenvalue is $m_{u_{L}}=\bar{\lambda}^{2} \chi^{2}\left(4 a^{2}+4 a^{\prime \prime 2}+\chi^{2}\right) / m_{u_{H}}$. The naive expectation for this quantity is $(\Lambda / M)^{3} \Lambda$.

The mass matrix for $E^{c} \sim(1,1,1)+$ h.c., in the $\left(A^{\prime \prime}, \mathbf{1 6}(\overline{\mathbf{1 6}}), A\right)$ basis is

$$
M_{E^{c}, \overline{E^{c}}}=\left(\begin{array}{ccc}
2 \mathcal{A} K\left(v^{2}+B^{2}\right)-2 \tilde{\lambda}_{7} \Lambda & 2 i \tilde{\lambda}_{4} \chi & i \bar{\lambda} \chi^{2} \\
-2 i \tilde{\lambda}_{4} \chi & -4 \tilde{\lambda}_{4} b^{\prime \prime} & -2 \bar{\lambda} b^{\prime \prime} \chi \\
-i \bar{\lambda} \chi^{2} & -2 \bar{\lambda} b^{\prime \prime} \chi & 5 \tilde{\lambda}_{9} s
\end{array}\right)
$$

Using the $F_{i}=0$ equations it can be verified that this mass matrix has one zero eigenvalue. The masses of the other two states are $5 \tilde{\lambda}_{9} s$ and $-\tilde{\lambda}_{4}\left(4 b^{\prime \prime}+\chi^{2} / b^{\prime \prime}\right)$, to lowest order in $\bar{\lambda} \Lambda$.

The mass matrix for the $Y \sim(3,2,-5 / 6)$ and $X \sim(\overline{3}, 2,5 / 6)$ fields is given in the $\left(A^{\prime \prime}, S, A\right)$ basis by

$$
M_{Y X}=\left(\begin{array}{ccc}
-2 \mathcal{A} K(u v-A B)+2 \tilde{\lambda}_{7} \Lambda & -2 i \mathcal{A} K(u B+v A) & 0 \\
-2 i \mathcal{A} K(u B+v A) & -2 \mathcal{A} K(u v-A B)+2 \tilde{\lambda}_{6} \Lambda & i \tilde{\lambda}_{9} a \\
0 & i \tilde{\lambda}_{9} a & -\frac{5}{2} \tilde{\lambda}_{9} s
\end{array}\right) .
$$

It can be verified, after some tedious algebra, that this matrix has one zero eigenvalue. This matrix is therefore rank 2 . The masses of the other two states are $O\left(\Lambda^{2} / M\right)$

The $Q \sim(\mathbf{3}, \mathbf{2}, 1 / 6)$ and $\bar{Q} \sim(\overline{\mathbf{3}}, \mathbf{2},-1 / 6)$ mass matrix, in the $\left(A^{\prime \prime}, S, \mathbf{1 6}(\overline{\mathbf{1 6}}), A\right)$ basis, is

$$
M_{Q \bar{Q}}=
$$




$$
\left(\begin{array}{cccc}
2 \mathcal{A} K(u v+A B)-2 \tilde{\lambda}_{7} \Lambda & -2 i \mathcal{A} K(A v-B u) & 2 i \tilde{\lambda}_{4} \chi-\bar{\lambda} a \chi & i \bar{\lambda} \chi^{2} \\
2 i \mathcal{A} K(A v-B u) & -2 \mathcal{A} K(u v+A B)+2 \tilde{\lambda}_{6} \Lambda & 0 & -i \tilde{\lambda}_{9} a \\
-2 i \tilde{\lambda}_{4} \chi-\bar{\lambda} a \chi & 0 & -2 \tilde{\lambda}_{4}\left(a^{\prime \prime}+b^{\prime \prime}\right) & -\bar{\lambda}\left(a^{\prime \prime}+b^{\prime \prime}\right) \chi \\
-i \bar{\lambda} \chi^{2} & i \tilde{\lambda}_{9} a & -\bar{\lambda}\left(a^{\prime \prime}+b^{\prime \prime}\right) \chi & \frac{5}{2} \tilde{\lambda}_{9} s
\end{array}\right)
$$

It can be verified that this matrix has at least one zero eigenvalue. To verify that it has only one zero eigenvalue, it is suffucient to verify that the coefficient of $O(e)$ in the expansion of $\operatorname{det}\left(M_{Q \bar{Q}}-e 1\right)$ is non-vanishing. Since the entries proportional to $\bar{\lambda}$ result in a tiny perturbation to the spectrum of $M_{Q \bar{Q}}$, it is suffucient to compute the $O(e)$ coefficient, call it $p$, while setting $\bar{\lambda}=0$. In this case it is

$$
\begin{aligned}
p= & 4 \mathcal{A} K \frac{(B u-A v)}{\left(u^{2}+A^{2}\right)\left(v^{2}+B^{2}\right)}\left(-2 \tilde{\lambda}_{4} \tilde{\lambda}_{6}\left(u B^{2}-(u-v) u v-A^{2} v\right) \Lambda\right. \\
& -\tilde{\lambda}_{7} \tilde{\lambda}_{9}\left(B\left(u^{2}+A^{2}\right)+A\left(v^{2}+B^{2}\right)\right) \Lambda \\
& -\tilde{\lambda}_{4} \tilde{\lambda}_{9}\left((A+B)^{2}+(u-v)^{2}\right) \\
& \left.+\mathcal{A} K\left(\tilde{\lambda}_{9}(A+B)-2 \tilde{\lambda}_{4}(u-v)\right)\left(u^{2}+A^{2}\right)\left(v^{2}+B^{2}\right)\right)
\end{aligned}
$$

If this vanishes at generic values for the couplings constants, then it must, in particular, vanish for two solutions and sets of couplings constants that are related by Eqns.(D.3) and (D.4). Under this scaling, however, $p \propto C \times\left(c_{1} g^{-2}+c_{2} g^{-4}\right)$, with $C, c_{1}$ and $c_{2}$ functions of the initial vevs and couplings. This vanishes only if either $C=0$ or $c_{1}=0$ and $c_{2}=0$. The first condition, $C=0$, implies $A v=B u$, whereas the second $c_{2}=0$ implies that $A+B=0$ and $u-v=0$. Either of these conditions over-constrain the vevs, so they will not be satisfied at 
a generic solution. In particular, $p=(0.07)^{3}$ for the numerical solution (I) given by Eqn.(D.1). The expected mass for the three massive eigenvalues is therefore $O\left(\Lambda^{2} / M\right)$

The mass matrix for the coloured adjoints $(8,1,0)$ in the $\left(A^{\prime \prime}, S, A\right)$ basis is

$$
M_{\mathbf{8 8}}=\left(\begin{array}{ccc}
-\mathcal{A} K\left(u^{2}-A^{2}\right)+\tilde{\lambda}_{7} \Lambda & -2 i \mathcal{A} K u A & 0 \\
-2 i \mathcal{A} K u A & -\mathcal{A} K\left(u^{2}-A^{2}\right)+\tilde{\lambda}_{6} \Lambda & i \tilde{\lambda}_{9} a \\
0 & i \tilde{\lambda}_{9} a & 0
\end{array}\right)
$$

The determinant is $\left(\tilde{\lambda}_{9} a\right)^{2}\left(\tilde{\lambda}_{7} \Lambda-\mathcal{A} K\left(u^{2}-A^{2}\right)\right)$ and is non-vanishing. The size of the three masses is expected to be $m_{8} \sim \Lambda^{2} / M$. For the numerical solution (I) in Eqn.(D.1), this determinant is $(0.05)^{3}$.

The mass matrix for the $S U(2)$ adjoints $(\mathbf{1}, \mathbf{3}, 0)$ in the $\left(A^{\prime \prime}, S, A\right)$ basis is

$$
M_{33}=\left(\begin{array}{ccc}
-\mathcal{A} K\left(v^{2}-B^{2}\right)+\tilde{\lambda}_{7} \Lambda & -2 i \mathcal{A} K v B & 0 \\
-2 i \mathcal{A} K v B & -\mathcal{A} K\left(v^{2}-B^{2}\right)+\tilde{\lambda}_{6} \Lambda & 0 \\
0 & 0 & -\frac{3}{2} \tilde{\lambda}_{9} s
\end{array}\right)
$$

The determinant is

$$
-3 \tilde{\lambda}_{9} s\left(\mathcal{A} K\left(\mathcal{A} K\left(v^{2}+B^{2}\right)^{2}=\left(\tilde{\lambda}_{6}+\tilde{\lambda}_{7}\right)\left(v^{2}-B^{2}\right) \Lambda\right)+\tilde{\lambda}_{6} \tilde{\lambda}_{7} \Lambda^{2}\right) / 2
$$

and is non-vanishing. The size of the three masses is expected to be $m_{3} \sim \Lambda^{2} / M$. For the numerical solution (I) in Eqn.(D.1), this determinant is $-(0.04)^{3}$.

The $S$ field contains $(\mathbf{6}, \mathbf{1}, 2 / 3)+$ h.c. and $(\mathbf{1}, \mathbf{3},-1)+$ h.c. These fields acquire Dirac masses $-\mathcal{A} K\left(u^{2}+A^{2}\right)$ and $-\mathcal{A} K\left(v^{2}+B^{2}\right)$, respectively. The $(\overline{3}, 1,1 / 3)+$ h.c. and $(\mathbf{1}, \mathbf{2},-1 / 2)+$ h.c. fields in the $\mathbf{1 6}+\overline{\mathbf{1 6}}$ acquire Dirac masses $-4 \tilde{\lambda}_{4}\left(a^{\prime \prime}+b^{\prime \prime}\right)$ and $-2 \tilde{\lambda}_{4}\left(3 a^{\prime \prime}+b^{\prime \prime}\right)$, respectively. 
Finally, there are 8 gauge singlets in this model. The quantum modified constraint implies that only 7 of these are independent. The quantum modified constraint can be used to solve for one of the gauge singlets. Of the remaining 7 , one of these is the Nambu-Goldstone boson multiplet of the $S O(10) \rightarrow S U(5)$ symmetry breaking. The mass matrix for the remaining 6 gauge singlets is rather cumbersome and is not presented here. For the numerical solution (I) presented at the start of this Appendix, it can be checked that the determinant of this matrix is $-6 \times 10^{-7}$ (in units of $\Lambda=1$.); the typical mass of each singlet is then $\sim 0.09 \Lambda$. 


\section{Bibliography}

[1] S. L. Glashow, Nucl. Phys. 22, (1961) 579; S. Weinberg, Phys. Rev. Lett. 19, (1967) 1264; A. Salam in Elementary Particle Theory, ed. N. Svartholm (Almqvist and Wiksell, Stockholm, 1968) p. 367.

[2] S. L. Glashow, J. Iliopoulos and L. Maiani, Phys. Rev. D2, (1970) 1285.

[3] N. Cabibbo, Phys. Rev. Lett. 10, (1963) 531; M. Kobayashi and T. Maskawa; Prog. Theor. Phys. 49, (1973) 652.

[4] See, for example, L. Susskind, Phys. Rev. D20, (1979) 2619.

[5] See, for example, J. Wess and J. Bagger, Supersymmetry and Supergravity, Princeton University Press, Princeton (1992); P. Fayet and S. Ferrara, Phys. Rep. 5, 249 (1977) 249; H.P. Nilles, Phys. Rep. 110, (1984) 1; M.F. Sohnius, Phys. Rep 128, (1985) 2; I. Hinchliffe, Ann. Rev. Nucl. Part. Sci. 36, (1986) 505.

[6] B. Zumino, Nucl. Phys. B89, (1975) 535; P. West, Nucl. Phys. B106, (1976) 219; M. Grisaru, W. Siegel and M. Roček, Nucl. Phys. B159, (1979) 429.

[7] R. Haag, J. T. Lopuszański and M. Sohnius, Nucl. Phys. B88, (1975) 257.

[8] L. Giradello and M. T. Grisaru, Nucl. Phys. B194, (1982) 65.

[9] M. Dine, R. Leigh and A. Kagan, Phys. Rev. D48 (1993) 4269. 
[10] R. Barbieri, L. Hall and G. Dvali, Phys. Lett. B377 (1996) 76; R. Barbieri and L. Hall, Nuovo Cim. 110A (1997) 1.

[11] M. Dine, W. Fischler and M. Srednicki, Nucl. Phys. B189, (1981) 575; C. Nappi and B. Ovrut, Phys. Lett. B113, (1982) 175; M. Dine and W. Fischler, Nucl. Phys. B204, (1982) 346; L. Alvarez-Gaume, M. Claudson and M. B. Wise, Nucl. Phys. B207, (1982) 96.

[12] Y. Nir and N. Seiberg, Phys. Lett. B309 (1993) 337.

[13] H. Georgi and S. L. Glashow, Phys. Rev. Lett. 32, (1974) 438.

[14] See, for example, G. G. Ross, Grand Unified Theories, Addison-Wesley, New York (1985), and the references therein.

[15] J. C. Pati and A. Salaam, Phy. Rev. D10 (1974) 1240.

[16] T. Appelquist and J. Carrazzone, Phy. Rev. 11 (1975) 2856.

[17] M. A. Shifman and A. I. Vainshtein, Nucl. Phys. B277, (1986) 456.

[18] V. A. Novikov, M. A. Shifman, A. I. Vainshtein and V. I. Zakharov, Nucl. Phys. B229, (1983) 381; M. A. Shifman, A. I. Vainshtein and V. I. Zakharov, Phys. Lett. B166, (1986) 334.

[19] N. Seiberg, Phys. Lett. B318, (1993) 469.

[20] N. Arkani-Hamed and H. Murayama, Phy. Rev. D57, (1998) 6638; N. ArkaniHamed and H. Murayama, LBNL-40460 (1997), hep-th/9707133. 
[21] K.G. Wilson and J. Kogut, Phys. Rep. 12, (1974) 75; J. Polchinski, Nucl. Phys. B231, (1984) 269.

[22] N. Arkani-Hamed and H. Murayama, private communication.

[23] M. Shifman and A. Vainshtein, Nucl. Phys. B296, (1988) 445; I. I. Kogan and M. Shifman, Phys. Rev. Lett. 75, (1995) 2085; I. I. Kogan, M. Shifman and A. Vainshtein, Phys. Rev. D53, (1996) 4526.

[24] P. Argyres, Introduction to Supersymmetry, Lecture Notes, unpublished.

[25] L.J. Hall and M. Suzuki, Nucl. Phys. B231, (1984) 419.

[26] C.E. Carlson, P. Roy and M. Sher, Phys. Lett. B357, (1995) 99.

[27] S. Dimopoulos and L.J. Hall, Phys. Lett. B207, (1987) 210.

[28] V. Barger, G.F. Giudice and T. Han, Phys. Rev D40, (1989) 2987.

[29] R. Godbole, P. Roy and X. Tata, Nucl. Phys. B401, (1993) 67.

[30] S. Dawson, Nucl. Phys. B261, (1985) 297.

[31] G. Bhattacharyya and D. Choudhury, Mod. Phys. Lett. A10, (1995) 1699.

[32] G. Bhattacharyya, J. Ellis and K. Sridhar, Mod. Phys. Lett. A10, (1995) 1583.

[33] R. Mohapatra, Phys. Rev D34, (1986) 3457.

[34] S. Abachi et al. Phys. Rev. Lett. 74, (1995) 2632. 
[35] F. Abe et al., Phys. Rev. Lett. 74, (1995) 2626.

[36] Caso et al., Particle Data Group: Review of Particle Physics, Euro. Phys. J. C3 (1998) 1.

[37] E. Ma and P. Roy, Phys. Rev D41, (1990) 988; E. Ma and D. Ng, Phys. Rev. D41, (1990) 1005.

[38] R. Barbieri and A. Masiero, Nucl. Phys. B267, (1986) 679.

[39] M. Leurer, Phys. Rev. Lett. 71, (1993) 1324.

[40] M.K. Gaillard and B.W. Lee, Phys. Rev. D10, (1974) 897.

[41] J. Shigemitsu, in Proceedings of the XXVII International Conference on High Energy Physics (Glasgow, Scotland, UK, July 1994), edited by P.J. Bussey and I.G. Knowles (Institute of Physics Publishing, Bristol and Philadelphia, 1995).

[42] C. Bernard, Nucl. Phys. (Proc. Suppl.) B34, (1994) 47; S. Sharpe, Lectures given at the Theoretical Advanced Study Institute in Particle Physics (TASI 94), Boulder, CO, 29 May - 24 Jun 1994.

[43] C. Albajar et al., Phys. Lett. B186, (1987) 247; H. Albrecht et al., Phys. Lett. B197, (1987) 452.

[44] T. Inami and C.S. Lim, Prog. Theor. Phys. 65, (1981) 292. 
[45] G. Martinelli, in Proceedings of the 6th Rencontres De Bois, Bois, France 20 - 25 Jun 1994.

[46] M. S. Atiya et al., Phys. Rev. Lett. 70, (1993) 2521; (erratum) M. S. Atiya et al., Phys. Rev. Lett. 71, (1993) 305.

[47] H. Dreiner and R.J.N. Phillips, Nucl. Phys. B367, (1991) 591.

[48] E. Berger and H. Contopanagos, Phys. Lett. B361, (1995) 115.

[49] Y. Grossman, Z. Ligeti and E. Nardi, Nucl. Phys. B465, (1996) 369; Erratumibid. B480, (1996) 753.

[50] N. Krasnikov, Phys.Lett. B388, (1996) 783.

[51] N. Arkani-Hamed et al., Phys. Rev. Lett. 77, (1996) 1937.

[52] N. Arkani-Hamed et al., Nucl. Phys. B505, (1997) 3.

[53] N. Krasnikov, JETP Lett. 65, (1997) 148.

[54] S. I. Bityukov and N. Krasnikov, hep-ph/9712358.

[55] A. Bartl et al., Proceedings of the 1996 DPF Summer Study (Snowmass, CO, 25 June - 12 July, 1996), LBNL-39413 (1996).

[56] I. Hinchliffe et al., Phys. Rev. D55, (1997) 5520; private communication with I. Hinchliffe. 
[57] See, for example, N. Arkani-Hamed, H.-C. Cheng and L. J. Hall, Nucl. Phys. B472, (1996) 95, and references therein.

[58] H. Baer et al., Phys. Rev. D49, (1994) 3283.

[59] F. Paige, communication at the conference.

[60] Y. Coadou et al., ATLAS Internal Note ATL-PHYS-98-126, LBNL-41757.

[61] See p.191-192 of chap.12 (Physics Performance) of the CMS Technical Proposal at http://cmsinfo.cern.ch/TP/TP.html.

[62] See Fig.3.42, p.98 of ATLAS Collaboration, Technical Proposal, CERN/LHCC/94-43, LHCC/P2 (1994).

[63] R. Barbieri and G. Giudice, Nucl. Phys. B306 (1988) 63.

[64] G. Anderson and D. Castaño, Phys. Lett. B347 (1995) 300.

[65] S. Dimopoulos and H. Georgi, Nucl. Phys. B193 (1981) 150.

[66] I. Affleck, M. Dine and N. Seiberg, Nucl. Phys. B256 (1985) 557.

[67] L. Hall, J. Lykken and S. Weinberg, Phys. Rev. D27 (1983) 2359.

[68] F. Gabbiani and A. Masiero, Nucl. Phys. B322 (1989) 235; J. S. Hagelin, S. Kelley and T. Tanaka, Nucl. Phys. B415 (1994) 293; F. Gabbiani, E. Gabrielli, A. Masiero and L. Silvestrini, Nucl. Phys. B477 (1996) 321.

[69] M. Dine, A. Nelson, Y. Nir and Y. Shirman, Phys. Rev. D53 (1996) 2658. 
[70] N. Arkani-Hamed, C. D. Carone, L. J. Hall and H. Murayama, Phys. Rev. D54 (1996) 7032.

[71] P. Ciafaloni and A. Strumia, Nucl. Phys. B 494 (1997) 41; G. Bhattacharyya and A. Romanino, Phys. Rev. D 55 (1997) 7015.

[72] P. Fayet, Nucl. Phys. B90 (1975) 104.

[73] R. Barbieri, G. Dvali and A. Strumia, Phys. Lett. B333 (1994) 79.

[74] M. Dine, A. Nelson and Y. Shirman, Phys. Rev. D51 (1995) 1362.

[75] I. Dasgupta, B. A. Dobrescu and L. Randall, Nucl. Phys. B483 (1997) 95.

[76] S. Dimopoulos, G. Giudice and A. Pomarol, Phys. Lett. B389 (1996) 37; S. Martin, Phys. Rev. D55 (1997) 3177.

[77] See for example, S. Dimopoulos, S. Thomas and J. Wells, Nucl. Phys. B 488 (1997) 39. and references therein.

[78] Talk presented by Glen Cowan (ALEPH collaboration) at the special CERN particle physics seminar on physics results from the LEP run at $172 \mathrm{GeV}, 25$ February, 1997.

[79] G.F. Giudice, M.L. Mangano, G. Ridolfi and R. Ruckel (convenors), Searches for New Physics, hep-ph/9602207.

[80] See, for example, J. Ellis, S. Kelley, and D. V. Nanopoulos, Nucl. Phys. B373 (1992) 55; P. Langacker and N. Polonsky, Phys. Rev. D47 (1993) 4028. 
[81] S. M. Barr, Phys. Rev. D 55 (1997) 6775.

[82] F. Wilczek, Phys. Rev. Lett. 40 (1978) 279.

[83] M. Carena and P. W. Zerwas (convenors), Higgs Physics, CERN Yellow Report CERN 96-01, hep-ph/9602250.

[84] W. Adam et al, DELPHI Collaboration, CERN-PPE/96-119.

[85] H.P. Nilles, M. Srednicki and D. Wyler, Phys. Lett. B124 (1983) 337; A.B. Lahanas, Phys. Lett. B124 (1983) 341.

[86] L. Ibanez and G. G-Zoss, Phys. Lett. B110, (1982) 215; K. Inoue, A. Kakuto, H. Komatsu and S. Takeshita, Prog. Theor. Phys. 68, (1982) 927; L. AlvarezGaumme, M. Claudson and M. Wise, Nucl. Phys. B207, (1982) 96.

[87] M. Dine, R. Leigh and A. Kagan, Phys. Rev. D48, (1993) 4269; P. Pouliot and N. Seiberg, Phys. Lett. B318, (1993) 169; D. B. Kaplan and M. Schmaltz, Phys. Rev. D49, (1994) 3741 ; L. J. Hall and H. Murayama, Phys. Rev. Lett. 75, (1995) 3985; N. Arkani-Hamed, H.-C. Cheng, L. J. Hall, Phys. Rev. D54, (1996) 2242; R. Barbieri and L. J. Hall, Nuovo Cim. 110A, (1997) 1; R. Barbieri, L. J. Hall, S. Raby and A. Romanino, Nucl. Phys. B493, (1997) 3.

[88] Y. Nir and N. Seiberg, Phys. Lett. B309, (1993) 337

[89] M. Dine, A. Kagan and S. Samuel, Phys. Lett. B243, (1990) 250. 
[90] A. Pomoral and D. Tommansini, Nucl. Phys. B466, (1996) 3.

[91] G. Dvali and A. Pomarol, Phys. Rev. Lett. 77, (1996) 3728; G. Dvali and A. Pomarol, Nucl. Phys. B522, (1998) 3.

[92] A.G. Cohen, D.B. Kaplan, and A.E. Nelson, Phys. Lett. B388, (1996) 588.

[93] S. Ambrosanio and A. Nelson, Phys. Lett. B411, (1997) 283.

[94] A. Nelson and D. Wright, Phys. Rev. D56, (1997) 1598.

[95] R. N. Mohapatra and A. Riotto, Phys. Rev. D55, (1997) 4262.

[96] S. Dimopoulos and G.F. Giudice, Phys. Lett. B357, (1995) 573.

[97] N. Arkani-Hamed and H. Murayama, Phys. Rev. D56, (1997) 6733.

[98] J. Bagger, K. T. Matchev, and R. Zhang, Phys. Lett. B412, (1997) 77.

[99] M. K. Gaillard and B. W. Lee, Phys. Rev. Lett. 33, (1974) 108.

[100] The Aleph Collaboration, pre-prints 856, EPS 619, 622, submitted to the 1997 EPS-HEP Jerusalem Conference, 19-26 Aug.

[101] W. Siegel, Phys. Lett. B84, (1979) 193.

[102] D. M. Capper, D. R. T. Jones and P. van Nieuwenhuizen, Nucl. Phys. B167, (1980) 479.

[103] I. Jack, D. R. T. Jones, S. Martin, M. Vaughn and Y. Yamada, Phys. Rev. D50, (1994) 5481. 
[104] S. Martin and M. Vaughn, Phys. Rev. D50, (1994) 2282.

[105] P. Ciafaloni and A. Strumia, Nucl. Phys. B494, (1997) 41; G. Bhattacharyya and A. Romanino, Phys. Rev. D55, (1997) 7015; K. Agashe and M. Graesser, Nucl. Phys. B507, (1997) 3.

[106] E. Poppitz and S. Trivedi, Phys. Lett. B401, 38 (1997).

[107] N. Seiberg, Phys. Lett. B318, (1993) 469; N. Seiberg, Phys. Rev. D 49, (1994) 6857.

[108] H.-C. Cheng, Phys. Lett. B410, (1997) 45.

[109] S. Dimopoulos, G. Dvali and R. Rattazzi, Phys. Lett. B410, (1997) 119.

[110] K. I. Izawa and T. Yanagida, Prog. Theor. Phys. 97, (1997) 913; K. I. Izawa and T. Yanagida, Prog. Theor. Phys. 99, (1998) 423.

[111] N. Arkani-Hamed, C. Carone, L. Hall and H. Murayama, Phys. Rev. D54, (1996) 7032.

[112] C. Carone, L. Hall and T. Moroi, Phys. Rev. D56, (1997) 7183.

[113] S. Dimopoulos and H. Georgi, Phys. Lett. B117, (1982) 287; K. Inoue, A. Kakuto and T. Takano, Prog. Theor. Phys. 75, (1986) 664; A. Anselm and A. Johansen, Phys. Lett. B200, (1988) 331; Z. Berezhiani and G. Dvali, Sov. Phys. Lebedev Inst. Rep. 5, (1989) 55; Z. Berezhiani, C. Csáki, L. Randall, Nucl. Phys. B444, (1995) 61. 
[114] S. Dimopoulos and F. Wilczek, in Proceedings of the Erice Summer School, edited by A. Zichichi (Publisher, City, 1981).

[115] L. Hall and U. Sarid, Phys. Rev. Lett. 70, (1993) 2673.

[116] See, for example, J. Ellis, S. Kelley, and D. V. Nanopoulos, Nucl. Phys. B373, (1992) 55; P. Langacker and N. Polonsky, Phys. Rev. D47, (1993) 4028 .

[117] R. Barbieri, G. Dvali, A. Strumia, Z. Berezhiani and L. Hall, Nucl. Phys. B432, (1994) 49.

[118] S. M. Barr, Phys. Rev. Lett. 79, (1997) 4748.

[119] K. S. Babu and S. M. Barr, Phys. Lett. B381, (1996) 137.

[120] K. S. Babu and S. M. Barr, Phys. Rev. D48, (1993) 5354.

[121] K. S. Babu and S. M. Barr, Phys. Rev. D50, (1994) 3529.

[122] J. Hisano, H. Murayama and T. Yanagida, Nucl. Phys. B 402, (1993) 46.

[123] P. Nath and R. Arnowitt, Phys. Rev. D38, (1988) 1479.

[124] G. Anderson, S. Raby, S. Dimopoulos, L. J. Hall and G. D. Starkman, Phys. Rev. D49, (1994) 3660; L. Hall and S. Raby, Phys. Rev. D51, (1995) 6524; K. S. Babu and S. M. Barr, Phys. Rev. Lett. 75, (1995) 2088. 